\title{
Can I still get pregnant?
}

Citation for published version (APA):

Hoenderboom, B. M. (2021). Can I still get pregnant? Long-term complications of Chlamydia trachomatis infections in women. [Doctoral Thesis, Maastricht University]. Ipskamp.

https://doi.org/10.26481/dis.20210701bh

Document status and date:

Published: 01/01/2021

DOI:

10.26481/dis.20210701bh

Document Version:

Publisher's PDF, also known as Version of record

\section{Please check the document version of this publication:}

- A submitted manuscript is the version of the article upon submission and before peer-review. There can be important differences between the submitted version and the official published version of record.

People interested in the research are advised to contact the author for the final version of the publication, or visit the DOI to the publisher's website.

- The final author version and the galley proof are versions of the publication after peer review.

- The final published version features the final layout of the paper including the volume, issue and page numbers.

Link to publication

\footnotetext{
General rights rights.

- You may freely distribute the URL identifying the publication in the public portal. please follow below link for the End User Agreement:

www.umlib.nl/taverne-license

Take down policy

If you believe that this document breaches copyright please contact us at:

repository@maastrichtuniversity.nl

providing details and we will investigate your claim.
}

Copyright and moral rights for the publications made accessible in the public portal are retained by the authors and/or other copyright owners and it is a condition of accessing publications that users recognise and abide by the legal requirements associated with these

- Users may download and print one copy of any publication from the public portal for the purpose of private study or research.

- You may not further distribute the material or use it for any profit-making activity or commercial gain

If the publication is distributed under the terms of Article $25 \mathrm{fa}$ of the Dutch Copyright Act, indicated by the "Taverne" license above, 


\section{Can I still get pregnant? \\ Long-term complications of}

Chlamydla trachomatis infections

\section{in women}

Bernice M Hoenderboom : 


\section{Can I still get pregnant? \\ Long-term complications of Chlamydia trachomatis infections in women}

Bernice M Hoenderboom 
ISBN: 978-94-6421-375-1

Lay-out: Wendy Bour-van Telgen

Printing: Ipskamp Printing, Enschede, the Netherlands

(c) Bernice Hoenderboom, 2021

All rights reserved. No part of this thesis may be reproduced or transmitted in any form or by any means without prior permission from the author. 


\title{
Can I still get pregnant? \\ Long-term complications of Chlamydia trachomatis infections in women
}

\author{
PROEFSCHRIFT \\ ter verkrijging van de graad van doctor aan de Universiteit Maastricht, \\ op gezag van de Rector Magnificus, prof. dr. Rianne M. Letschert \\ volgens het besluit van het College van Decanen, \\ in het openbaar te verdedigen
}

op donderdag 1 juli 2021 om 16.00 uur

door

Bernice Maria Hoenderboom 


\section{Promotoren}

Prof. dr. S.A. Morré

Prof. dr. J.E.A.M. van Bergen (Amsterdam UMC)

\section{Copromotoren}

Dr. B.H.B. van Benthem (RIVM)

Dr. S. Ouburg (Amsterdam UMC)

\section{Beoordelingscommissie}

Prof. dr. P.H.M. Savelkoul (voorzitter)

Prof. dr. R.F.P.M. Kruitwagen

Prof. dr. D.D.M. Braat (Radboudumc)

Dr. Y. Pannekoek (Amsterdam UMC) 


\section{Table of content}

Chapter 1 General introduction 9

The risk of complications following chlamydia infection: Setting up a cohort study

Chapter 2 The Netherlands Chlamydia Cohort Study (NECCST) protocol to assess the risk of late complications following Chlamydia trachomatis infection in women

BMC Infectious Diseases 2017, 10.1186/s12879-017-2376-y

Chapter 3 Chlamydia trachomatis antibody detection in home-collected blood samples for use in epidemiological studies Journal of Microbiological Methods 2018, 10.1016/j.mimet.2017.11.022

Chapter 4 The role of single nucleotide polymorphisms in the TLR2, NOD1, CXCR5, and IL10 genes in the susceptibility to and severity of Chlamydia trachomatis infections Pathogens 2021; 10.3390/pathogens10010048

\section{The value of Chlamydia trachomatis serology}

Chapter 5 Antibody testing in estimating past exposure to Chlamydia trachomatis in the Netherlands Chlamydia Cohort Study Microorganisms 2019, 10.3390/microorganisms7100442

Chapter 6 Chlamydia trachomatis whole proteome microarray analysis of the 103 Netherlands Chlamydia Cohort study Microorganisms 2019, 10.3390/microorganisms7120703

\section{Long-term complications of chlamydia infections and predictive factors}

Chapter 7 Relation between Chlamydia trachomatis infection and pelvic inflammatory disease, ectopic pregnancy, and tubal factor infertility in a Dutch cohort of women previously tested for chlamydia in a chlamydia screening trial Sexually Transmitted Infections 2019; 10.1136/sextrans-2018-053778 
Chapter 8 Pregnancies and time to pregnancy in women with and without a previous

Chlamydia trachomatis infection

Sexually Transmitted Diseases, 47(11), 739-747.10.1097/

OLQ.0000000000001247

Chapter 9 General discussion

Chapter 10 Summary

202

Samenvatting

205

Impact Paragraph

209

Curriculum Vitae

211

List of publications

212

Affiliation list

213

Dankwoord 



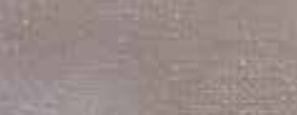

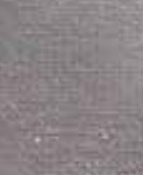

\section{sisting}

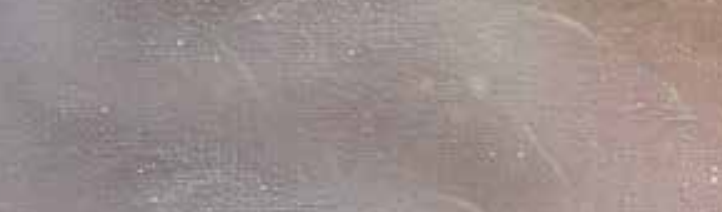

जince

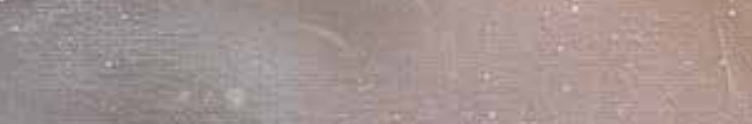

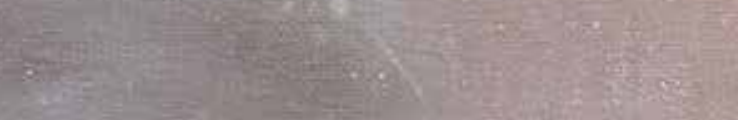

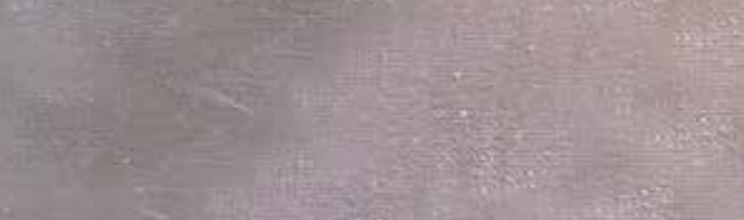

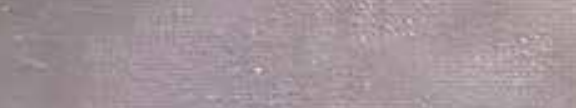

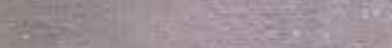

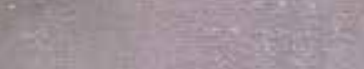

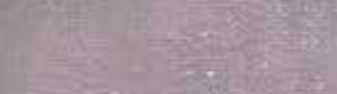

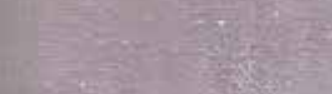

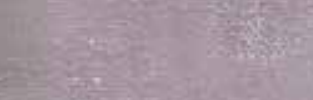

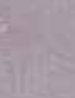

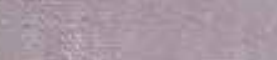

netwo

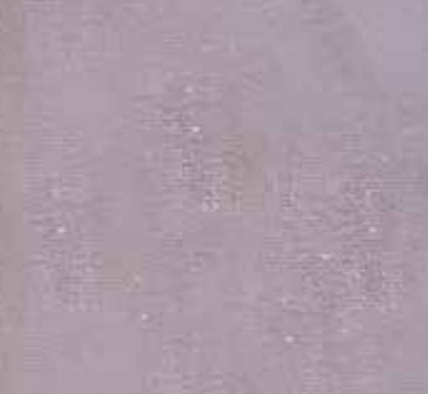

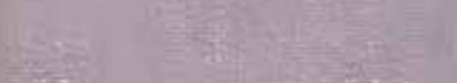

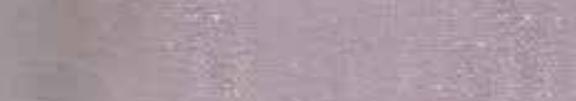


General introduction 


\section{General introduction}

"Chlamydia trachomatis is one of the most enigmatic pathogens known to medical science"(1). The pathogen was discovered in 1907, but evidence suggest that chlamydial disease is already known for centuries. In ancient Egyptian and Chinese texts references were made to diseases of the eye as early as $1550 \mathrm{BC}(2)$. Despite a century of intensive research, specified after the discovery of the genital form (in 1976) and the introduction of nucleic acid amplification tests (NAATs) in the mid 1990's, Chlamydia trachomatis (hereafter referred to as "chlamydia") remains the most reported bacterial sexually transmitted infection (STI) and we still do not have an answer how to handle this bacterial infection(1). Every year around 127 million new chlamydia infections occur(3) of which women bear a disproportional burden. Specifically because of the severe complications such as pelvic inflammatory disease (PID), ectopic pregnancy, chronic pelvic pain (CPP) and tubal factor infertility (TFI) that might develop in women following chlamydia infection.

\section{Chlamydia trachomatis}

The chlamydia bacterium is a gram-negative, obligate intracellular pathogen that infect eukaryotic cells. This intracellular reproduction cycle is unique for prokaryotes, therefore after chlamydia was first discovered it was believed to be a protozoa or virus. After the achievement of growing the chlamydiae in embryonated eggs in 1957 and cell culture in 1965 the discussion whether it was a virus or bacteria was resolved $(1,4)$. Based on mono- and polyclonal antibodies against the major outer membrane protein (MOMP) chlamydia can be subdivided in three biovars: trachoma (serovars A-C), urogenital (D-K) and lymphogranuloma venereum (LGV) which is L1, L2, L2a, L3(5). The developmental cycle of chlamydiae includes two highly distinct forms: Elementary bodies (EB) and reticulate bodies (RB), Figure 1. EBs are the infectious stage. EBs infect the host epithelial cell by endocytosis and prevent fusion of lysosomes with chlamydia-containing phagosome, thereby permitting intracellular survival. After the endocytosis EBs transform into the vegetative and noninfectious form of RBs. These RBs divide every 2-3 hours by binary fission for 18-72 hours. By filling the endosome with RBs (100-1,000 RBs) the inclusion bodies will become visible with antibody-specific stain in the host cell. Following this process the RBs differentiate into new infectious EBs. These EBs are then released from the host cell either by rupture of the host cell or exocytosis $(1,6)$. 


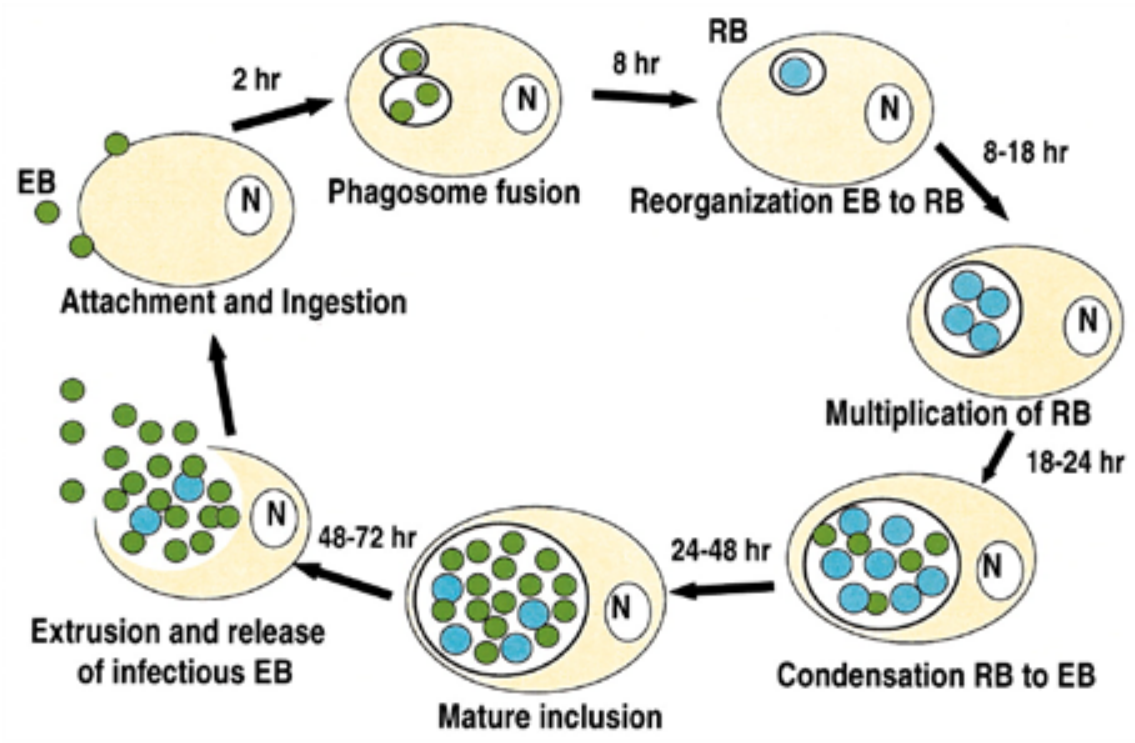

Figure 1 Chlamydia trachomatis reproduction cycle. Figure from Hammerschlag et al. 2004(7).

As chlamydia is a sexually transmitted infection, transmission occurs mainly through sexual intercourse with mucosal contact. However, transmission via genital-to-eye contact by hands and during birth from mother to child are also possible. Transmission rate for women when having sexual intercourse with infected men was estimated between 13-65\%, however for asymptomatic infections transmission rates per sex act as low as 2-5\% have been estimated(8-11).

\section{Clinical manifestations}

A urogenital chlamydia infection is mostly asymptomatic, about $70 \%$ of infections in women are asymptomatic and $50 \%$ in men(12,13). When women do have symptoms these include abnormal vaginal discharge, dysuria, and post-coital or intermenstrual bleeding. Symptomatic men present with urethral discharge, dysuria, and sometimes with testicular pain(14). If infections are left untreated, most of these infections will resolve spontaneously with no complications. Clearing rates of asymptomatic infections were found to be $50 \%$ in one year and $95 \%$ after four years(15, 16). However, in women infections can ascend to the upper genital tract which can result in severe reproductive tract complications(17). Chlamydia infections that ascend from the vagina of the cervix to the upper genital tract can affect the reproductive tract, i.e. endometrium, fallopian tubes, ovaries, or pelvic peritoneum. An infection induced inflammation in the female reproductive tract is called a PID(18). The infection can result in fibrinous or suppurative inflammatory damage along the epithelial surface or peritoneal surface of the fallopian tubes and ovaries. This might lead to scarring, adhesions, and partial or total obstruction of the fallopian tubes(18). Scarring, adhesions and obstructions can cause ectopic pregnancies and tubal factor infertility. A prior chlamydia infection may also prolong time to pregnancy in women without any visible tubal pathology, due 
to damage to the tubal mucosa (compromising embryo transport) especially the ciliated mucosal cells or unfavourable effects in the endometrium (affecting implantation) (19).

\section{Immune response}

The female reproductive tract contains crucial cells of both the innate and adaptive immune systems that detect and respond to a chlamydia infection, and these cells are found throughout the vagina, cervix, uterus, and fallopian tubes(20).

The innate immune system, a non-specific system, is the first line of defense against unknown pathogens. This first-line defense is executed by neutrophils, natural killers cells, macrophages, and dendritic cells. Next to these immune cells, epithelial cells are suggested to play an important role in the early immune response(21). Natural killer cells and neutrophils are first recruited at the site of infection(22). Neutrophils are able to inactivate chlamydia in vitro(23). Natural killer cells, although primarily involved in viral infections and cancer, also play an important role in in the early elimination of intracellular bacteria $(24,25)$. Natural killer cells produce interferon gamma which is important in inhibiting the growth of chlamydia(26) and induce a Th1 immune response(22). Macrophages migrate to the infection site, phagocytose bacteria, and produce proinflammatory cytokines $(27,28)$. Furthermore, macrophages autophagy enhances antigen presentation to $T$ cells(29) and may have an effect on infection by inducing T cell death. Last, dendritic cells are the most important antigen presenting cells. Immature dendritic cells are highly phagocytic, and degrade after internalization of the bacteria components and present these to $T$ cells via major histocompatibility complex. Presentation of peptides to T cells initiate a cell mediated or humoral immune response(22, 30, 31).

Major cellular components of the adaptive immune response are T cells and B cells. T cells depend on the help of antigen presenting cells to recognize either pathogens or antigens. T cells recognize chlamydia antigens such as Outer membrane protein 2 (Omp2)(32), polymorphic outer membrane protein D (pmpD)(33), MOMP(34), heat shock protein 60 (hsp60)(35), and chlamydial protease activating factor (CPAF)(36). Chlamydia can induce a Th2-associated response, but the Th1 immune response predominates. A Th1 response is characterized by the production of interleukin-12 by antigen presenting cells(37) and the following initiation of interferon producing $T$ cells and plasma B cells. T cells and plasma B cells then secrete Th1-associated antibodies(22, 38,39 ), Figure 2. Numerous chlamydia antigens can induce antibody responses which play a role in protective immunity. B cells might be primarily important for secondary infection opposed to initial infections $(40,41)$.

To conclude, for the initial clearance of a chlamydia infection, cell-mediated immunity that activates neutrophils, natural killer cells and macrophages is required. For protective immunity both cellmediated and humoral immunity are needed(22). 


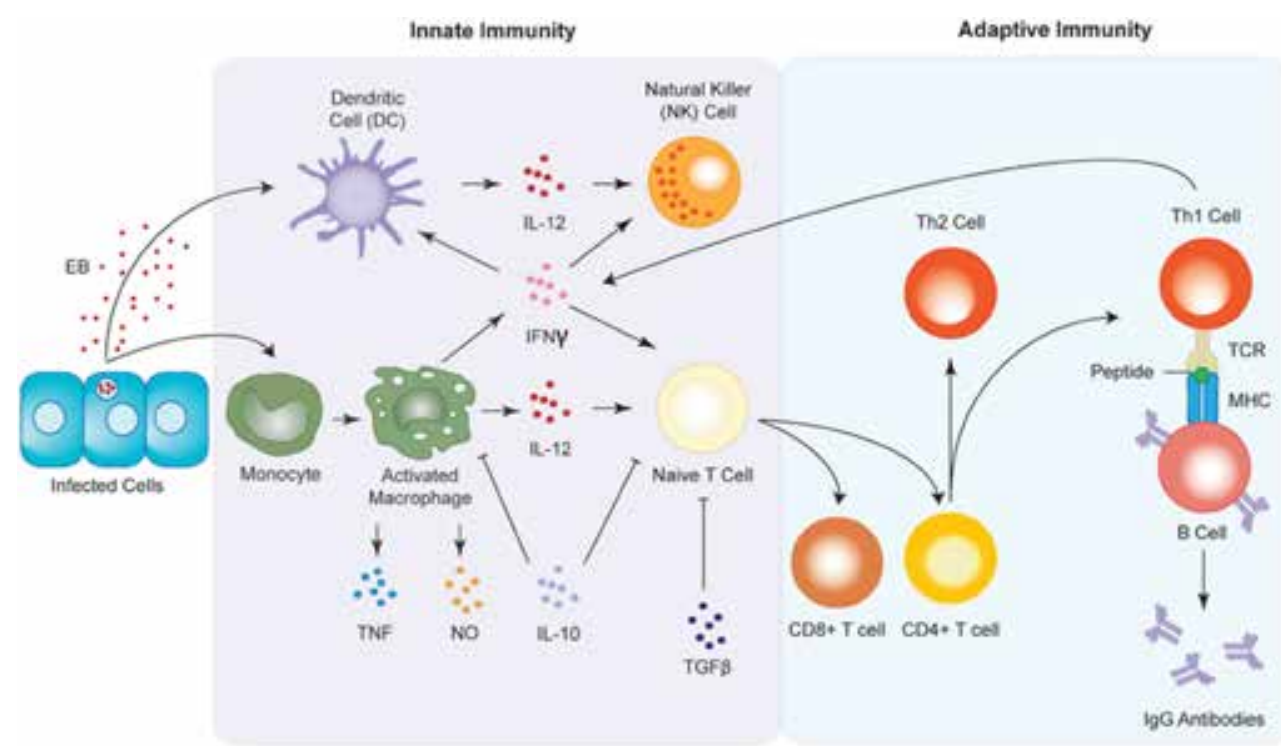

Figure 2 Immune response to Chlamydia infection: Innate and adaptive response. At time of infection macrophages and dendritic cells (antigen presenting cells) are sequestered to the site of infection and release pro-inflammatory cytokines such as IFNy and IL-12. These chemokines activate natural killer cells and induce the maturation of T cells into CD8+ or CD4+ T cells. In turn CD4+ T cells form to either T-helper 1 or T-helper 2 type T cells. T-helper 1 cells interrelate with B cells via the T cell receptor and the major histocompatibility complex to create antibodies against the chlamydia infection. Figure from Redgrove et al., 2014(42).

\section{Detection and treatment}

Detection and treatment of the infection is straightforward. Detection of the Chlamydia trachomatis bacterium is done with NAATs on cervical swabs, rectal swabs, pharyngeal swabs or urine samples. NAATs have a high sensitivity and specificity to detect the chlamydial bacterium(43). European guidelines recommend treatment of uncomplicated urogenital (non-LGV) and pharyngeal infections with 1 gram of azithromycin and for rectal infections 100mg doxycycline twice a day for seven days(44). A test of cure is not recommended. Recent updated guidelines from British Association for Sexual Health and HIV (BASHH) recommend doxycycline over azithromycin for uncomplicated chlamydial infections(45). A similar guideline change in European guidelines might be expected. In contrast to current infections, detection of past infections is less straightforward. Methods to detect chlamydia antibodies have been available for decades. Serology can be used to investigate chlamydia population prevalence and serve as a biomarker of chlamydial pathology having found its use in the triage of sub-fertile women attending at the department of gynecology if they did not get pregnant in one year. But despite the potential and the availability, the use of serological assays for clinical and epidemiological purposes remained limited due to questions about the performance and interpretation of chlamydia serological assays(46). For example the sensitivity of 
which the range is 30-93\% depending on 'golden standard' and assays used(47). Nevertheless, chlamydia serology can be useful in detecting previous infections which otherwise would have been missed.

\section{Interpatient variability}

The clinical course of a chlamydia infection and development of complications differ greatly between women. Pathogen factors, epidemiological context, host factors (immune response and genetics) all play individually and in combination an important role in this course(48).

\section{Pathogen factors}

Many studies have been performed to assess a possible association between the different chlamydia serovars and repeat infection or clinical course of infection. In most countries, including the Netherlands, serovars $E$ and $F$ are predominant(49-51). From several studies it is suggested that serovars $D$ and I are associated with asymptomatic infection and serovar $G$ with a more symptomatic course of infection $(52,53)$. Serovar $E$ and D were found to give the highest IgG responses(54). In mouse models it was found that serovars $E$ and $D$ had the longest infections duration and serovars $\mathrm{H}$ and I the shortest(55). Although these associations are primarily linked to a symptomatic course of infection and not directly to late complications, these results suggest that different serovars may contribute to chlamydial pathogenesis(48).

\section{Epidemiological context}

It is well established that several epidemiological factors contribute to the risk for late complications. First are repeat infections, which represent a substantial proportion of all detected chlamydia infections. Repeat diagnosis of chlamydia might be due to reinfections, treatment failures, or persistent infections(48). The rate of reinfections is high, between $20-30 \%$ and associated with sexual risk behavior(56-58). The risk for PID, ectopic pregnancy and TFI increases after reinfections (59-61). Repeat chlamydia infections increase the risk for PID a further $20 \%$ and every repeated PID doubles the risk for $\operatorname{TFI}(62,63)$. Although these reinfections might be due to sexual risk behavior or treatment failure the rise of reinfections over time might also be caused by another determinant. It is proposed that because of increased testing and treating the development of natural immunity is impaired. The consequence might be that people are more at risk for a repeat infection. This is called the arrested immune hypothesis, which states that reinfections and late complications are less likely to occur in women who have spontaneously cleared their initial infection and have therefore some protective immunity $(48,64,65)$. Next to chlamydia reinfections, co-infections might increase the risk for sequelae. Co-infections with gonorrhea did not increase the risk for PID additively(66), but bacterial vaginosis during a chlamydia infection was associated with an increased risk for PID(67).

Furthermore, young age is associated with contracting a chlamydia infection. The highest incidence is found among 15-19 years old's $(68,69)$, however sexual risk behavior increases with age, this might indicate a development of a partial protective immune response(70). PID rates however 
increase with older age(71). Sexual risk behavior in itself, such as a younger age at sexual debut, a higher number of lifetime partners, and previous STIs increased the risk for late complications(48, 72). It is however difficult to determine if this is a direct effect or an indirect effect due to the risk of multiple infections.

\section{Host factors}

\section{Host immune response}

As described previously in "Immune response" multiple studies about the immune response to a chlamydial infection support a predominant Th1 or cytotoxic profile or immune response. Although this Th1 immune response is needed to resolve the infection, it can also result in tissue pathology and late complications(73). This is called the immunological paradigm for chlamydia pathogenesis. Previously it was thought that the underlying core of this paradigm was delayedtype hypersensitivity or autoimmune molecular mimicry, but in 2003 the 'cellular paradigm' was introduced as a basis for the immunological paradigm(48, 73). This paradigm of chlamydia pathogenesis states that the host response to chlamydia is initiated and sustained by epithelial and endothelial cells, the primary home for chlamydia(74). The inflammatory processes are necessary and sufficient to account for chronic and intense inflammation and the promotion of cellular proliferation, tissue remodeling and scarring(73). It is however unclear why a Th1 profile results in either clearance of an infection or pathology.

Host genetic factors

The cellular immune response to a chlamydia infection might be subject to host genetic influences. The degree and mechanisms of such genetic control have important implications for understanding of chlamydia pathogenesis(75). Results from a twin study from a trachoma endemic area about chlamydia immunogenetics suggested that host genetic factors contributed to almost $40 \%$ of the variation in the immune response to chlamydia(76). The scarring of the eye and the scarring of the ovarian tubes have a remarkable immunogenetic resemblance (75), and therefore it is expected to find a similar effect of host genetics on tubal pathology. Previous studies investigating the role of variation in the genetic predisposition in genital chlamydia showed increased or decreased risks for late complications(77). For example single nucleotide polymorphisms (SNPs) in tolllike receptors (TLRs), which are essential in recognizing pathogenic components, can have increased and decreased risks for developing tubal factor infertility. In haplotype analyses the distribution of TLR9 haplotype -1486 T (rs187084), -1237 C (rs5743836), +1174 G (rs352139), and $+2848 \mathrm{~A}$ ( $\mathrm{rs352140)}$ were found more frequently in women who developed tubal pathology. The TLR2 haplotype $1(-16934 \mathrm{~T} /+2477 \mathrm{G})$ had an protective effect on the development of tubal pathology(78). A different example is a 32-bp depletion within the CCR5 gene. CCR5 (Chemokine Receptor Type 5) is a chemokine receptor present on several immune cells. The 32-bp deletion results in an alteration of the function due to premature termination of the protein(79). Barr et al. found that when both alleles were mutated, this resulted in a protective effect against developing tubal pathology(80). However, these results could not be reconfirmed (81). Many other variations in genes have been associated with chlamydia disease outcome, however it is often difficult to 
confirm previous findings. Nevertheless, it is clear that host genetic factors play a role in the susceptibility to and severity of chlamydia infection(77).

\section{Epidemiology of chlamydia}

With an estimated 127 million new infections in 2016 worldwide, chlamydia remains the most reported bacterial STI (3). The estimated pooled prevalence of chlamydia in 15-49 year old women was 3.8\% (95\% Cl 3.3-4.5) and 2.7\% (95\% Cl 1.9-3.7) in men. Regional values ranged from 1.5 to $7.0 \%$ in women and $1.2 \%-4.0 \%$ in men(3). Upper-middle and lower income countries had the highest chlamydia prevalence's.

In the Netherlands, there are an estimated 60,000 new chlamydia infections each year $(82,83)$. In the past two decades, three studies estimated the chlamydia population prevalence in the Netherlands. This was 2.0\% (95\% Cl 1.7 to 2.3) in 2002/2003 among 16-29 year old's, 2.7\% between 2008-2011 among 16-29 year old's and 2.8\% (95\% Cl 1.5\% to 5.2\%) in 2016/2017 among 18-34 year old's, all within each other's $95 \%$ confidence interval $(82,84,85)$. At the Sexual Health Centers $(\mathrm{SHC})$, detailed information of clients who are tested for chlamydia about characteristics and sexual risk behavior is collected. Sixty percent of all chlamydia infections is diagnosed among heterosexual men and women under 25 years of age. Percentage positive is highest among people notified for chlamydia (32\%). High positivity rates were also seen among heterosexual men with symptoms, lower educated men and women and having had and STI in the previous year(68). SHCs focus on risk groups, that is why chlamydia positivity is much higher compared to the general population, i.e. $15 \%$ in women and $18 \%$ in heterosexual men. Due to a stronger focus on risk groups through recent years the positivity rate increased from $11 \%$ in 2009 to $15 \%$ in 2016 in women and $11 \%$ in 2009 to $18 \%$ in 2016 in men.(68). Despite this increase in test positivity, population prevalence's as described above remained similar over the last two decades. In a population based chlamydia serology study, seroprevalence was 9\% among women and 6\% among men in 2007 in the age of 15-39(86). Given a test sensitivity for detecting past infections from about $38 \%$ measured in a previous study, true proportion of people ever infected with chlamydia might be around $23 \%$ for women and $15 \%$ for men(47). This high proportion might result in many women affected by chlamydia related complications.

Estimations for chlamydia complications risks ranged widely. Estimated risks of PID following a chlamydia infection varied between $0.5 \%$ and $72 \%$ depending on the study population and the definitions used (87-92). Subfertility as a result of prior chlamydia infection was estimated to occur in $0.1-6 \%$ of women infected $(87,93)$, and $0-1 \%$ of women with a prior infection may develop an ectopic pregnancy $(94,95)$. Because of these wide ranges, questions remained about the exact risk for a complication following an infection.

\section{Chlamydia control}

The aim of chlamydia control is to reduce chlamydia prevalence and morbidity. (Cost-) Effectiveness of control activities are directly linked to the height of the infection prevalence and the size of the risk for complications(89). For example the number needed to screen is lower when a risk size is higher and visa versa. This endorses the importance of accurate risk estimates. The rationale 
for screening is that detection of asymptomatic infections will allow early treatment and prevent onward transmission and complications(96). Chlamydia control activities vary greatly within European countries. ECDC performed a systematic survey of chlamydia control activities in Member States in 2012. Six countries (21\%) did not have any organized chlamydia control activity, whereas thirteen countries (46\%) had opportunistic testing and only one had a screening program, the UK. Chlamydia control in the Netherlands is characterized as opportunistic testing(97). Easy access to healthcare is implemented in the Netherlands. Regular STI care is provided by general practitioners (GPS), SHC and in case needed by a medical specialist. The majority of chlamydia tests is done by GPs. In addition to GPs, SHC subsidized by the Ministry of Health, Welfare and Sports, provide low threshold, free-of-charge STI/HIV testing and care, targeted at high-risk groups. Risk groups are currently defined 1) People with STI related symptoms, 2) Notified for an STI, 3) Men who have sex with men (MSM), 4) Originating from an HIV/STI endemic area, 5) Reporting a partner from an HIV/STI endemic area of MSM, 6) Aged under 25, 7) Sex worker or last 8) Being a victim of sexual violence. Through the years, mainly because of financial constraints, SHC have prioritized populations at highest risk of an STI(68).

\section{Chlamydia screening programme's}

As mentioned above the UK, as the only country in Europe, implemented in 2002 the National Chlamydia Screening Programme (NCSP). The NCSP recommends all sexually active youngsters until the age of 25 to be tested annually for genital chlamydia infection or on change of partner. $A$ re-test should be offered three months following a positive test. Screening is arranged within a range of providers such as GPs, pharmacies, contraception services, sexual health and reproductive services and pregnancy termination services $(98,99)$. Annually between 1.5 to 2 million chlamydia tests are being undertaken and about one in every three women is tested. Overall positivity was around eight percent in 2015(100).

To determine if a chlamydia screening could be effective in the Netherlands, a chlamydia prevalence pilot was performed in 2002/2003. The aim of the pilot was to examine the chlamydia population prevalence and the feasibility of a screening programme through general practices and municipal public health services (MHS). Because of low prevalence's found in rural populations, targeted approaches and prioritizing high risk area's was suggested. Home based testing organized by MHS in close cooperation with regular care was feasible. It was advised to expand the body of evidence before a new nationwide screening should be implemented(84). In 2008 the Chlamydia Screening Implementation (CSI) study started. The objectives were to investigate the effect of yearly invitations for chlamydia screening on the percentage of positive chlamydia tests, uptake of chlamydia screening and to estimate impact on chlamydia prevalence among sexually active women and men in the Netherlands. In three regions in the Netherlands: Amsterdam, Rotterdam and South Limburg, over 300,000 women and men aged 16-29 years participated between 2008 and 2011 in the CSI. Participation rate was however low, 16\% in the first round and only 10\% percent in the third round. Prevalence did not substantially decline in the intervention blocks compared to the controls blocks (0.2\% difference.) which resulted in an odds ratio of $0.96(95 \% \mathrm{Cl} 0.83-1.10)$. It was concluded that there was no statistical evidence of an impact on chlamydia positivity rates 
or estimated population prevalence from the CSI programme. The evidence did not support a national roll out of a register based chlamydia screening programme(85).

Effectiveness of current chlamydia control measures on prevalence and complications In the Netherlands it was decided to forgo a national chlamydia screening programme because of limited impact on chlamydia prevalence in the 'real world'. And also for the English National Chlamydia Screening Programme (NSCP), there is no evidence of effectiveness on population prevalence(88). A Cochrane review determining the effect of chlamydia screening programmes on prevalence reduction concluded a lack of evidence(101). Moreover, the Australian Chlamydia Control Effectiveness Pilot (ACCEPt), that investigated the effect of opportunistic testing in primary care clinics on the prevalence of chlamydia infections, concluded that "sizeable reductions in chlamydia prevalence might not be achievable"(102).

There is evidence of an impact of chlamydia screening on PID reduction in women in the Cochrane review. However, the size of the effect is uncertain due to biases in included studies which might exaggerated the effect. Studies with high risk of bias detected an average of $58 \%$ reduction in PID following chlamydia screening, compared to $20 \%$ (non-significant) reduction in studies with a low risk of bias. Only one included trial determined the effect of screening on female infertility and ectopic pregnancy. However, for both outcomes, no impact was found. None of the included trials included harms of screening(101). In the ACCEPt trial, results showed a reduction in hospital PID in the intervention group of $40 \%$. However, no reduction was seen in clinic PIDs. The conclusion was that a substantial reduction in chlamydia associated complications might again not be achievable(102). In the evaluation of the NSCP it was concluded that monitoring frequency of PID and ectopic pregnancies did not provide clear evidence of an impact of chlamydia screening on these disease outcomes in England to date(100).

To conclude, evidence of an impact of chlamydia screening or opportunistic testing on chlamydia prevalence reduction is lacking. There is some evidence of an impact of chlamydia screening on PID reduction, but a substantial impact is questionable. Last, there is no evidence of an impact of screening on infertility and ectopic pregnancy or harms of screening. What will be the future of chlamydia control?

\section{Determining risks for chlamydia related complications: the Netherlands Chlamydia Cohort Study (NECCST)}

To sum up, chlamydia remains the most reported bacterial STI worldwide as well as in the Netherlands despite comprehensive research in how to address this bacterial infection and extensive chlamydia control measures. In women these infections can lead to severe complications resulting in infertility. It can be concluded that in real world settings there is no evidence of an effect or substantial reduction of chlamydia screening program's and opportunistic testing on chlamydia prevalence. It might therefore be more effective to focus on prevention of complications following infection instead of prevention of infections. However, estimates of complications rates following infection as described above range widely; incidences of complications from chlamydia infections and its determinants have not been clearly established. Current estimates were based on studies using several different designs all with associated strengths and limitations, Table 1. 
Table 1. Study designs that were performed to assess complication rates following chlamydia infection

\begin{tabular}{|c|c|}
\hline Study design & Strenghts \\
\hline $\begin{array}{l}\text { Randomised controlled } \\
\text { trial }(R C T)(103,104)\end{array}$ & $\begin{array}{l}\text { Properly executed RCTs have } \\
\text { the highest level of evidence } \\
\text { (barring systematic reviews and } \\
\text { meta-analyses) and are the } \\
\text { 'golden standard' for ascertaining } \\
\text { effectiveness of treatment or } \\
\text { intervention. Bias in results can } \\
\text { be avoided by randomization } \\
\text { and blinding of participants or } \\
\text { researchers(105). }\end{array}$ \\
\hline
\end{tabular}

Limitations

RCTs are not the most useful study designs for long-follow up. Which, in chlamydia complication research is highly needed. The longest follow-up in RCT in chlamydia research was only one year. This excludes studying ectopic pregnancy and infertility as endpoints(104). Another major limitation in chlamydia research lies in ethical grounds. With an effective treatment available one cannot withhold participants in control groups from treatment (in or outside study context)(103).

Last to be able to find statistical significant results, large sample sizes are needed.

Retrospective cohort studies $(59,62,66)$

Registers based studies often include $>100.000$ people, chlamydia test results performed within the country are all registered and medical events such as PID, ectopic pregnancy and TFI are all diagnosed and registered. Large sample sizes and including registered events increase reproducibility and validity. Last, time has already passed and data is available.

Case-control studies (108-110)
The great advantages of casecontrol studies is the possibility to study rare diseases. Which applies for ectopic pregnancies and tubal factor infertility. For cohort studies, a large sample size is needed to find a sufficient amount of events for statistical analyses. This does not apply for case-control studies in which cases are the starting point. Furthermore, multiple factors can be studied at once. The power of studies can be increased by including more controls, for example a ratio of $1: 3$ (cases:controls)
Main limitations of registry based studies are that necessary information may be unavailable, confounder information is lacking, there is missing information on data quality, truncation at start of follow-up making it difficult to differentiate between prevalent and incident cases and the risk of data dredging(106). Last, despite the longitudinal data, only associations, not causations can be made(107).

The major limitation of a case-control study is the retrospective nature which often results in recall bias. No causal relation can be concluded only associations, and these studies are prone to confounding bias(111). Last, to include a sufficient amount of cases, the study population in chlamydia related infertility includes women from infertility clinics. Controls should be matched and are therefore also from infertility clinics. Although this is best for matching cases and controls, it is difficult to apply the results for the general population. 


\begin{tabular}{|c|c|c|}
\hline Study design & Strenghts & Limitations \\
\hline $\begin{array}{l}\text { Cross-sectional studies } \\
(112,113)\end{array}$ & $\begin{array}{l}\text { Cross-sectional studies are ideally } \\
\text { suited for prevalence studies or } \\
\text { to infer causation. By estimating } \\
\text { prevalence among groups with } \\
\text { and without and outcome of } \\
\text { interest a possible relation can } \\
\text { be explored. Advantages are that } \\
\text { participants are not deliberately } \\
\text { exposed, treated, or not treated } \\
\text { and hence there are seldom } \\
\text { ethical difficulties. Data are } \\
\text { collected only once and multiple } \\
\text { outcomes can be studied; which } \\
\text { makes this type of study relatively } \\
\text { cheap. }\end{array}$ & $\begin{array}{l}\text { The greatest disadvantage is the lack } \\
\text { of differentiating cause and effect } \\
\text { from simple association. Furthermore, } \\
\text { cross-sectional design do not provide } \\
\text { explanations for the findings. Last it is } \\
\text { difficult to study rare diseases, even } \\
\text { with large sample sizes one may find } \\
\text { zero cases(114). }\end{array}$ \\
\hline $\begin{array}{l}\text { Mathematical modeling } \\
\text { studies }(90-93,115, \\
116)\end{array}$ & $\begin{array}{l}\text { These type of studies are not } \\
\text { a preferred design to measure } \\
\text { causal relations or associations. } \\
\text { Nevertheless, these studies } \\
\text { are useful in trying to estimate } \\
\text { risks for complications following } \\
\text { chlamydia infection using } \\
\text { previously published data from } \\
\text { RCTs and observational studies. } \\
\text { In these models multiple data } \\
\text { sources can be combined and } \\
\text { complex disease mechanisms } \\
\text { are simplified. }\end{array}$ & $\begin{array}{l}\text { However in simplifying mechanisms, } \\
\text { important factors might be missed. } \\
\text { Furthermore, models depend on the } \\
\text { availability and representativeness of } \\
\text { input data(117). }\end{array}$ \\
\hline
\end{tabular}
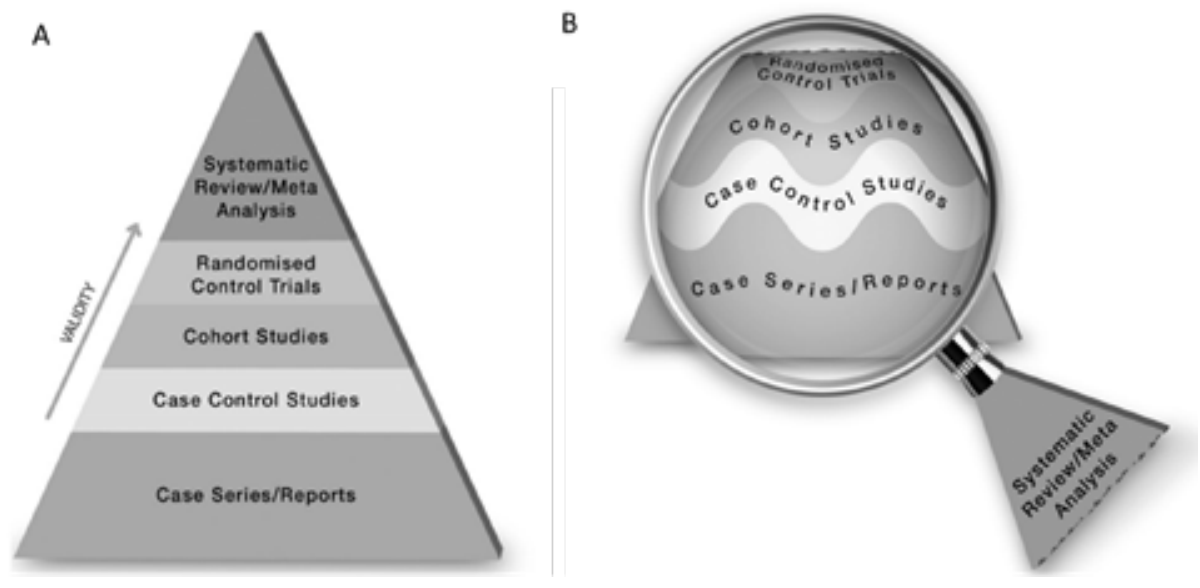

Figure 3. Evidence pyramid. The higher in the pyramid the higher the validity and rigorousness of study designs (A). Systematic reviews and meta-analysis are nowadays seen as a lens trough which evidence is viewed and applied in medical care (B). Figure from Murad et al. 2016 (118) 
Determining the risk of a previous chlamydia infection on long-term complications as PID, ectopic pregnancy or TFI is a well-known and a widely acknowledged challenge(119, 120). The best design to determine the risk for late complications and the effectiveness of treatment would be a randomized controlled trial, see Figure 3 . However, to expose women to a chlamydia infection reframe them from treatment and follow them over years of time to study disease progression to ectopic pregnancy or TFI is neither ethical nor feasible. Cohort studies are the design of choice in case randomization are not ethical and feasible and long follow-up is needed(114, 121). Prospective cohort studies are considered the gold standard among observational studies $(122,123)$. Due to the design and because of temporal sequence both incidence rate and cumulative incidences can be measured as well as risk ratio's, hazard ratio's and attributable risks. Multiple exposures can be studied and the risk for encountering survival bias and recall bias is lower $(124,125)$. Until now the risk of complications, specifically ectopic pregnancy and TFI, following a chlamydia infection longer than one year has not been assessed directly in a prospective cohort study. Therefore, to estimate the risk for late complications following a chlamydia infection and to gain insight in factors that contribute to the development of complications, the Netherlands Chlamydia Cohort Study (NECCST) was initiated. In this cohort study, the following aims will be addressed:

\section{Primary objective:}

- $\quad$ To quantify the incidence of PID, ectopic pregnancy and tubal factor subfertility and compare time to pregnancy in women with and without a previous chlamydia infection, in order to estimate the inherent risk of these outcomes by a previous chlamydia infection.

\section{Secondary objectives:}

- $\quad$ To explore which combination of host genetic biomarkers are able to distinguish women with a high risk of developing chlamydia related complications from women with a low risk of developing these complications.

- To determine demographic, behavioural, serological and infection-related factors that are associated with reproductive tract complications due to a preceding chlamydia infection.

\section{Challenges in a prospective cohort study}

Limitations to be taken into account while conducting a prospective cohort study are first the possibility of selection bias. Selection bias may affect the generalizability of the results also known as the external validity $(121,124,126)$. Second, officially causal relations may not be established based on cohort studies, however because of the longitudinal design they may aid in studying causal hypothesis $(121,127)$. Third, often a large sample size is needed with long follow-up(128). Furthermore in prospective cohort studies loss to follow up of participants will occur, which might become a problem if the loss to follow up is differential among exposed and non-exposed participants(129). Prospective cohorts are often time- and resource consuming, which makes it a costly research design(123). Besides general limitations and challenges of prospective cohort studies, there are specific challenges in chlamydia complication studies. First is misclassification in 
chlamydia history due to the asymptomatic nature of the infection (about 80\%(12)). Highly sensitive and specific Nucleic Acid Amplification Tests (NAATs) can identify the presence of chlamydia bacterial DNA in urine samples, vaginal swabs and rectal swabs, but presence of bacterial DNA is not per definition an infection since NAATs do not depend on viable pathogens(43). But far more important, NAATs can only detect current infections in the lower genital tract. Chlamydia infections are self-limiting, within a year $50 \%$ of people have cleared the infection(15). Many infections are therefore missed. Second, the diagnosis of PID is difficult. There is no consensus about the criteria of upper genital tract chlamydia disease and no accurate non-invasive diagnostics tests(120). PIDs are often based on lower abdominal and cervical signs and symptoms with poor sensitivity and specificity. Moreover, about two-thirds of PID are sub-clinical and cannot be diagnosed at all(18). Additionally, the diagnosis is prone for diagnostic bias; PID might be more easily diagnosed among women previously tested positive for chlamydia because of prior information bias(119). Third, a long-term follow-up to study chlamydia related complications is needed. The highest chlamydia incidence is in women between the age of 16-24(68). But complications often only become apparent when women starting to get pregnant. In the Netherlands a mean age of first pregnancy is 29 according to Statistics Netherlands (CBS). Last, incidences of ectopic pregnancy as well as TFI are low $(93,95)$. Therefore, a large sample size is needed to be able to test for significant differences between chlamydia positive and negative tested women.

\section{NECCST best}

Taking into account all limitations from an observational study design and challenges in chlamydia complication research, this cohort study will be a 'NECCST BEST' design following a RCT in determining the risk for PID, ectopic pregnancy, TFI and (prolonged) time to pregnancy. NECCST is a longitudinal cohort study and a follow-up study of the CSI study performed between 2008 and 2011. Women who participated in the CSI at least once and who consented to be approached for further research were re-invited for NECCST in 2015/2016. Major advantages from this construction were first that all women were previously tested at least once for chlamydia trachomatis infection in vaginal swab or urine sample. Second, CSI included over thousands of women in the age between 16 and 29. By inviting these women again we not only would end up with a large sample size, long follow-up, but also age would have increased to 20-37 at the beginning of NECCST and will be 26-43 at end of NECCST, which is ten years of follow-up. For a large majority of the cohort this will be sufficient to capture the age when chlamydia infections occur most often as well as the age women try to become pregnant.

\section{Aims and outline of this thesis}

This thesis aims on gaining the insight in the risks and risk factors for chlamydia related complications by studying a unique cohort that consists of women of reproductive age.

Part 1. The risk of complications following chlamydia infection: Setting up the cohort study

- $\quad$ Chapter 2 The Netherlands Chlamydia Cohort Study (NECCST) protocol to assess the risk of late complications following Chlamydia trachomatis infection in women describes the protocol 
for the NECCST study. Objectives, methods, and the power calculation are described and discussed in detail.

- Chapter 3 Chlamydia trachomatis antibody detection in home-collected blood samples for use in epidemiological studies evaluates the use of blood samples collected at home and sent to the laboratory for chlamydia antibody detection. Stability of chlamydia antibodies in blood samples processed within recommended two hours, three days and seven days was assessed.

- $\quad$ Chapter 4 The role of single nucleotide polymorphisms in the TLR2, NOD1, CXCR5, and IL10 genes in the susceptibility to and severity of Chlamydia trachomatis infections assessed four SNPs in five different cohorts to assess the risk or protection of four SNPs in the course of chlamydia infections.

Part 2. The value of Chlamydia trachomatis serology

- Chapter 5 Antibody testing in estimating past exposure to Chlamydia trachomatis in the Netherlands Chlamydia Cohort Study assessed the contribution of testing for chlamydia antibodies in the overall chlamydia lifetime prevalence to reduce misclassification in chlamydia status. In addition, factors associated with having chlamydial antibodies were assessed.

- Chapter 6 Chlamydia trachomatis whole proteome microarray analysis of the Netherlands Chlamydia Cohort study describes the use of whole proteome analyses that allows for the identification of novel informative antigens as general infection markers or chlamydial disease associated antigens.

Part 3. Long-term complications of chlamydia infections and predictive factors

- Chapter 7 Relation between Chlamydia trachomatis infection and pelvic inflammatory disease, ectopic pregnancy, and tubal factor infertility in a Dutch cohort of women previously tested for chlamydia in a chlamydia screening trial assessed the association between chlamydia infection and related complications as PID, ectopic pregnancy and TFI in all women participating in the NECCST study. Additionally, factors associated with an increased risk were determined.

- $\quad$ Chapter 8 Pregnancy rates and time to pregnancy in women with and without a previous Chlamydia trachomatis infection aimed to assess pregnancy rates and time to pregnancy among women with a previous chlamydia infection compared to women without and participating in the NECCST. 


\section{References}

1. Black CM. Chlamydial Infection: A Clinical and Public Health Perspective: Karger; 2013.

2. Taylor HR. Trachoma: A Blinding Scourge from the Bronze Age to the Twenty-first Century: Centre for Eye Research Australia; 2008.

3. Rowley J, Vander Hoorn S, Korenromp E, Low N, Unemo M, Abu-Raddad LJ, et al. Chlamydia, gonorrhoea, trichomoniasis and syphilis: global prevalence and incidence estimates, 2016. Bull World Health Organ. 2019;97(8):548-62P.

4. Gordon FB, Quan AL. Isolation of the Trachoma Agent in Cell Culture. Proc Soc Exp Biol Med. 1965;118:354-9.

5. Newhall WJt, Terho P, Wilde CE, 3rd, Batteiger $\mathrm{BE}$, Jones RB. Serovar determination of Chlamydia trachomatis isolates by using type-specific monoclonal antibodies. J Clin Microbiol. 1986;23(2):333-8.

6. Elwell C, Mirrashidi K, Engel J. Chlamydia cell biology and pathogenesis. Nat Rev Microbiol. 2016;14(6):385-400.

7. Hammerschlag MR. Chlamydia trachomatis and Chlamydia pneumoniae infections in children and adolescents. Pediatr Rev. 2004;25(2):43-51.

8. Lin JS, Donegan SP, Heeren TC, Greenberg M, Flaherty EE, Haivanis R, et al. Transmission of Chlamydia trachomatis and Neisseria gonorrhoeae among men with urethritis and their female sex partners. J Infect Dis. 1998;178(6):1707-12.

9. Tu W, Ghosh P, Katz BP. A Stochastic Model for Assessing Chlamydia trachomatis Transmission Risk Using Longitudinal Observational Data. J R Stat Soc Ser A Stat Soc. 2011;174(4):975-89.

10. Althaus CL, Low, N. P1-S5.02 Towards more robust estimates of the per sex act transmission probability of Chlamydia trachomatis. Sexually Transmitted Infections. 2011.

11. Althaus $\mathrm{CL}$, Turner KM, Schmid BV, Heijne JC, Kretzschmar M, Low N. Transmission of Chlamydia trachomatis through sexual partnerships: a comparison between three individual-based models and empirical data. J R Soc Interface. 2012;9(66):136-46.

12. Peipert JF. Clinical practice. Genital chlamydial infections. N Engl J Med. 2003;349(25):242430.

13. Farley TA, Cohen DA, Elkins W. Asymptomatic sexually transmitted diseases: the case for screening. Prev Med. 2003;36(4):502-9.

14. Christerson L, de Vries HJ, Klint M, Herrmann $B$, Morre SA. Multilocus sequence typing of urogenital Chlamydia trachomatis from patients with different degrees of clinical symptoms. Sex Transm Dis. 2011;38(6):490-4.

15. Morre $S A$, van den Brule AJ, Rozendaal L, Boeke AJ, Voorhorst FJ, de Blok S, et al. The natural course of asymptomatic Chlamydia trachomatis infections: 45\% clearance and no development of clinical PID after one-year follow-up. Int J STD AIDS. 2002;13 Suppl 2:128.

16. Molano M, Meijer CJ, Weiderpass E, Arslan A, Posso $\mathrm{H}$, Franceschi $\mathrm{S}$, et al. The natural course of Chlamydia trachomatis infection in asymptomatic Colombian women: a 5-year follow-up study. J Infect Dis. 2005;191(6):90716.

17. O'Connell CM, Ferone ME. Chlamydia trachomatis Genital Infections. Microb Cell. 2016;3(9):390-403.

18. Brunham RC, Gottlieb SL, Paavonen J. Pelvic inflammatory disease. $\mathrm{N}$ Engl $\mathrm{J}$ Med. 2015;372(21):2039-48.

19. Coppus SF, Land JA, Opmeer BC, Steures P, Eijkemans MJ, Hompes PG, et al. Chlamydia trachomatis IgG seropositivity is associated with lower natural conception rates in ovulatory subfertile women without visible tubal pathology. Hum Reprod. 2011;26(11):3061-7

20. Hafner L, Beagley K, Timms P. Chlamydia trachomatis infection: host immune responses and potential vaccines. Mucosal Immunol. 2008;1(2):116-30.

21. 21. Rasmussen SJ, Eckmann L, Quayle AJ, 
Shen L, Zhang YX, Anderson DJ, et al. Secretion of proinflammatory cytokines by epithelial cells in response to Chlamydia infection suggests a central role for epithelial cells in chlamydial pathogenesis. J Clin Invest. 1997;99(1):77-87.

22. Vasilevsky S, Greub G, Nardelli-Haefliger D, Baud D. Genital Chlamydia trachomatis: understanding the roles of innate and adaptive immunity in vaccine research. Clin Microbiol Rev. 2014;27(2):346-70.

23. Register KB, Morgan PA, Wyrick PB. Interaction between Chlamydia spp. and human polymorphonuclear leukocytes in vitro. Infect Immun. 1986;52(3):664-70.

24. Cheng M, Chen Y, Xiao W, Sun R, Tian Z. NK cellbased immunotherapy for malignant diseases. Cell Mol Immunol. 2013;10(3):230-52.

25. Shegarfi H, Sydnes K, Lovik M, Inngjerdingen M, Rolstad B, Naper C. The role of natural killer cells in resistance to the intracellular bacterium Listeria monocytogenes in rats. Scand $\mathrm{J} 34$. Immunol. 2009;70(3):238-44.

26. Beatty WL, Belanger TA, Desai AA, Morrison RP, Byrne GI. Tryptophan depletion as a mechanism of gamma interferon-mediated chlamydial persistence. Infect Immun. 1994;62(9):3705-11.

27. Bas $S$, Neff $L$, Vuillet $M$, Spenato $U$, Seya $T, 35$ Matsumoto $\mathrm{M}$, et al. The proinflammatory cytokine response to Chlamydia trachomatis elementary bodies in human macrophages is partly mediated by a lipoprotein, the macrophage infectivity potentiator, through 36 TLR2/TLR1/TLR6 and CD14. J Immunol. 2008;180(2):1158-68.

28. Yilma AN, Singh SR, Fairley SJ, Taha MA, Dennis VA. The anti-inflammatory cytokine, interleukin-10, inhibits inflammatory mediators in human epithelial cells and mouse macrophages exposed to live and UV- 37. inactivated Chlamydia trachomatis. Mediators Inflamm. 2012;2012:520174.

29. Crotzer VL, Blum JS. Autophagy and its role in MHC-mediated antigen presentation. J Immunol. 2009;182(6):3335-41.

30. Matyszak MK, Young JL, Gaston JS. Uptake and processing of Chlamydia trachomatis by human dendritic cells. Eur J Immunol. 2002;32(3):742-51.

31. Ojcius DM, Bravo de Alba Y, Kanellopoulos JM, Hawkins RA, Kelly KA, Rank RG, et al. Internalization of Chlamydia by dendritic cells and stimulation of Chlamydia-specific T cells. J Immunol. 1998;160(3):1297-303.

32. Goodall JC, Beacock-Sharp H, Deane KH, Gaston JS. Recognition of the 60 kilodalton cysteine-rich outer membrane protein OMP2 by $\mathrm{CD} 4(+) \mathrm{T}$ cells from humans infected with Chlamydia trachomatis. Clin Exp Immunol. 2001;126(3):488-93.

33. Goodall JC, Yeo G, Huang M, Raggiaschi R, Gaston JS. Identification of Chlamydia trachomatis antigens recognized by human CD4+ T lymphocytes by screening an expression library. Eur $\mathrm{J}$ Immunol. 2001;31(5):1513-22.

34. Kim SK, Devine L, Angevine M, DeMars R, Kavathas PB. Direct detection and magnetic isolation of Chlamydia trachomatis major outer membrane protein-specific CD8+ CTLs with HLA class I tetramers. J Immunol. 2000;165(12):7285-92.

35. Deane KH, Jecock RM, Pearce JH, Gaston JS. Identification and characterization of a DR4-restricted T cell epitope within chlamydia heat shock protein 60. Clin Exp Immunol. 1997;109(3):439-45.

36. Li W, Murthy AK, Chaganty BK, Guentzel MN, Seshu J, Chambers JP, et al. Immunization with dendritic cells pulsed ex vivo with recombinant chlamydial protease-like activity factor induces protective immunity against genital chlamydiamuridarum challenge. Front Immunol. 2011;2:73.

7. Chen L, Lei L, Zhou Z, He J, Xu S, Lu C, et al. Contribution of interleukin-12 p35 (IL-12p35) and IL-12p40 to protective immunity and pathology in mice infected with Chlamydia muridarum. Infect Immun. 2013;81(8):2962-71.

38. O'Connell CM, Ingalls RR, Andrews CW, Jr., Scurlock AM, Darville T. Plasmid-deficient 
Chlamydia muridarum fail to induce immune pathology and protect against oviduct disease. J Immunol. 2007;179(6):4027-34.

39. Olivares-Zavaleta N, Whitmire W, Gardner D, Caldwell HD. Immunization with the attenuated plasmidless Chlamydia trachomatis L2(25667R) strain provides partial protection in a murine model of female genitourinary tract infection. Vaccine. 2010;28(6):1454-62.

40. Morrison SG, Morrison RP. A predominant role for antibody in acquired immunity to chlamydial genital tract reinfection. J Immunol. 2005;175(11):7536-42.

41. Murthy AK, Chaganty BK, Li W, Guentzel MN, Chambers JP, Seshu J, et al. A limited role for antibody in protective immunity induced by 51 rCPAF and CpG vaccination against primary genital Chlamydia muridarum challenge. FEMS Immunol Med Microbiol. 2009;55(2):271-9.

42. Redgrove KA, McLaughlin EA. The Role of the Immune Response in Chlamydia trachomatis Infection of the Male Genital Tract: A DoubleEdged Sword. Front Immunol. 2014;5:534.

43. Meyer T. Diagnostic Procedures to Detect Chlamydia trachomatis Infections. Microorganisms. 2016;4(3).

44. Lanjouw E, Ouburg S, de Vries HJ, Stary A, Radcliffe K, Unemo M. 2015 European guideline on the management of Chlamydia trachomatis infections. Int J STD AIDS. 2016;27(5):333-48.

45. HIV BAfSHa. BASHH guidelines management 2018 [Available from: https://www. bashhguidelines.org/current-guidelines/ urethritis-and-cervicitis/chlamydia-2015/.

46. Woodhall SC, Gorwitz RJ, Migchelsen SJ, 54 Gottlieb SL, Horner PJ, Geisler WM, et al. Advancing the public health applications of Chlamydia trachomatis serology. Lancet Infect Dis. 2018;18(12):e399-e407.

47. Horner PJ, Wills GS, Reynolds R, Johnson AM, Muir DA, Winston A, et al. Effect of time since exposure to Chlamydia trachomatis on chlamydia antibody detection in women: a cross-sectional study. Sex Transm Infect. 2013;89(5):398-403.
48. Menon S, Timms P, Allan JA, Alexander K, Rombauts L, Horner $P$, et al. Human and Pathogen Factors Associated with Chlamydia trachomatis-Related Infertility in Women. Clin Microbiol Rev. 2015;28(4):969-85.

49. Xiong $L$, Kong F, Zhou H, Gilbert GL. Use of PCR and reverse line blot hybridization assay for rapid simultaneous detection and serovar identification of Chlamydia trachomatis. J Clin Microbiol. 2006;44(4):1413-8.

50. Jurstrand $M$, Falk $L$, Fredlund $H$, Lindberg $M$, Olcen $\mathrm{P}$, Andersson $\mathrm{S}$, et al. Characterization of Chlamydia trachomatis omp1 genotypes among sexually transmitted disease patients in Sweden. J Clin Microbiol. 2001;39(11):3915-9.

51. Quint KD, van Doorn LJ, Kleter B, de Koning MN, van den Munckhof HA, Morre SA, et al. A highly sensitive, multiplex broad-spectrum PCR-DNA-enzyme immunoassay and reverse hybridization assay for rapid detection and identification of Chlamydia trachomatis serovars. J Mol Diagn. 2007;9(5):631-8.

52. Gao $X$, Chen XS, Yin YP, Zhong MY, Shi MQ, Wei $W H$, et al. Distribution study of Chlamydia trachomatis serovars among high-risk women in China performed using PCR-restriction fragment length polymorphism genotyping. J Clin Microbiol. 2007;45(4):1185-9.

53. Lan J, Melgers I, Meijer CJ, Walboomers JM, Roosendaal R, Burger $\mathrm{C}$, et al. Prevalence and serovar distribution of asymptomatic cervical Chlamydia trachomatis infections as determined by highly sensitive PCR. J Clin Microbiol. 1995;33(12):3194-7.

54. Verweij SP, Lanjouw E, Bax CJ, Quint KD, Oostvogel PM, Dorr PJ, et al. Serovar D and $E$ of serogroup $B$ induce highest serological responses in urogenital Chlamydia trachomatis infections. BMC Infect Dis. 2014;14:3.

55. Ito JI, Jr., Lyons JM, Airo-Brown LP. Variation in virulence among oculogenital serovars of Chlamydia trachomatis in experimental genital tract infection. Infect Immun. 1990;58(6):20213.

56. Walker J, Tabrizi SN, Fairley CK, Chen MY, 
Bradshaw CS, Twin J, et al. Chlamydia trachomatis incidence and re-infection among young women--behavioural and microbiological characteristics. PLoS One. 2012;7(5):e37778.

57. Gaydos CA, Wright C, Wood BJ, Waterfield G, Hobson S, Quinn TC. Chlamydia trachomatis reinfection rates among female adolescents seeking rescreening in school-based health centers. Sex Transm Dis. 2008;35(3):233-7.

58. Geisler WM, Lensing SY, Press CG, Hook EW, 3rd. Spontaneous resolution of genital Chlamydia trachomatis infection in women and protection from reinfection. $J$ Infect Dis. 2013;207(12):1850-6.

59. Low $N$, Egger $M$, Sterne JA, Harbord RM, Ibrahim F, Lindblom B, et al. Incidence of severe reproductive tract complications associated with diagnosed genital chlamydial infection: the Uppsala Women's Cohort Study. Sex Transm Infect. 2006;82(3):212-8.

60. Davies B, Turner KME, Frølund M, Ward H, May MT, Rasmussen S, et al. Risk of reproductive complications following chlamydia testing: a population-based retrospective cohort study in Denmark. The Lancet Infectious Diseases. 2016;16(9):1057-64.

61. Hillis SD, Owens LM, Marchbanks PA, 70 Amsterdam LF, Mac Kenzie WR. Recurrent chlamydial infections increase the risks of hospitalization for ectopic pregnancy and pelvic inflammatory disease. Am J Obstet Gynecol. 1997;176(1 Pt 1):103-7.

62. Davies B, Turner KM, Frolund M, Ward H, May MT, Rasmussen S, et al. Risk of reproductive complications following chlamydia testing: a population-based retrospective cohort study in Denmark. Lancet Infect Dis. 2016.

63. Westrom L, Joesoef R, Reynolds G, Hagdu A, Thompson SE. Pelvic inflammatory disease and fertility. A cohort study of 1,844 women with laparoscopically verified disease and 657 control women with normal laparoscopic results. Sex Transm Dis. 1992;19(4):185-92.

64. Brunham RC, Pourbohloul B, Mak S, White $\mathrm{R}$, Rekart ML. The unexpected impact of a Chlamydia trachomatis infection control program on susceptibility to reinfection. J Infect Dis. 2005;192(10):1836-44.

65. Brunham RC, Rekart ML. The arrested immunity hypothesis and the epidemiology of chlamydia control. Sex Transm Dis. 2008;35(1):53-4.

66. Reekie J, Donovan B, Guy R, Hocking JS, Kaldor JM, Mak DB, et al. Risk of Pelvic Inflammatory Disease in Relation to Chlamydia and Gonorrhea Testing, Repeat Testing, and Positivity: A Population-Based Cohort Study. Clin Infect Dis. 2018;66(3):437-43.

67. Taylor BD, Darville T, Haggerty CL. Does bacterial vaginosis cause pelvic inflammatory disease? Sex Transm Dis. 2013;40(2):117-22.

68. Slurink IALVA, F.; Op de Coul, E.L.M.; Heijne, J.C.M.; van Wees, D.A.; Hoenderboom, B.M.; Visser, M;, den Daas, C.; Woestenberg, P.J.; Götz, H.M.; Nielen, M.; van Sighem, A.I.; Benthem, B.H.B Sexually transmitted infections in the Netherlands in 2018. 2019.

69. Paul KJ, Garcia PJ, Giesel AE, Holmes KK, Hitti JE. Generation C: prevalence of and risk factors for chlamydia trachomatis among adolescents and young women in Lima, Peru. J Womens Health (Larchmt). 2009;18(9):1419-24.

70. Rekart ML, Brunham RC. Epidemiology of chlamydial infection: are we losing ground? Sex Transm Infect. 2008;84(2):87-91.

71. Bender N, Herrmann B, Andersen B, Hocking JS, van Bergen J, Morgan J, et al. Chlamydia infection, pelvic inflammatory disease, ectopic pregnancy and infertility: cross-national study. Sex Transm Infect. 2011;87(7):601-8.

72. Hay PE, Kerry SR, Normansell R, Horner PJ, Reid $F$, Kerry SM, et al. Which sexually active young female students are most at risk of pelvic inflammatory disease? A prospective study. Sex Transm Infect. 2016;92(1):63-6.

73. Stephens RS. The cellular paradigm of chlamydial pathogenesis. Trends Microbiol. 2003;11(1):44-51.

74. 74. Darville T, Hiltke TJ. Pathogenesis of genital tract disease due to Chlamydia trachomatis. J Infect Dis. 2010;201 Suppl 2:S114-25. 
75. Morre SA, Karimi O, Ouburg S. Chlamydia trachomatis: identification of susceptibility markers for ocular and sexually transmitted infection by immunogenetics. FEMS Immunol Med Microbiol. 2009;55(2):140-53.

76. Bailey RL, Natividad-Sancho A, Fowler A, Peeling RW, Mabey DC, Whittle $\mathrm{HC}$, et al. Host genetic contribution to the cellular immune response to Chlamydia trachomatis: Heritability estimate from a Gambian twin study. Drugs Today (Barc). 2009;45 Suppl B:45-50.

77. Lal JA, Malogajski J, Verweij SP, de Boer $P$, Ambrosino E, Brand $A$, et al. Chlamydia trachomatis infections and subfertility: opportunities to translate host pathogen genomic data into public health. Public Health Genomics. 2013;16(1-2):50-61.

78. Karimi O, Ouburg $S$, de Vries HJ, Pena AS, Pleijster J, Land JA, et al. TLR2 haplotypes in the susceptibility to and severity of Chlamydia trachomatis infections in Dutch women. Drugs Today (Barc). 2009;45 Suppl B:67-74.

79. Mueller A, Strange PG. The chemokine receptor, 87 CCR5. Int J Biochem Cell Biol. 2004;36(1):35-8.

80. Barr EL, Ouburg S, Igietseme JU, Morre SA, Okwandu E, Eko FO, et al. Host inflammatory response and development of complications of Chlamydia trachomatis genital infection in CCR5-deficient mice and subfertile women with the CCR5delta32 gene deletion. J Microbiol Immunol Infect. 2005;38(4):244-54.

81. Laisk $T$, Peters $M$, Saare M, Haller-Kikkatalo $\mathrm{K}$, Karro H, Salumets A. Association of CCR5, TLR2, TLR4 and MBL genetic variations with genital tract infections and tubal factor infertility. J Reprod Immunol. 2010;87(1-2):74-81.

82. Heijne JCM, van den Broek IVF, Bruisten SM, van Bergen JEA, de Graaf H, van Benthem BHB. National prevalence estimates of chlamydia and gonorrhoea in the Netherlands. Sex Transm Infect. 2019;95(1):53-9.

83. Op de Coul EL, Gotz HM, Van Bergen J, 91. Fennema JS, Hoebe CJ, Koekenbier RH, et al. Who participates in t he Dutch Chlamydia screening? A study on demographic and behavioral correlates of participation and positivity. Sex Transm Dis. 2012;39(2):97-103.

84. van Bergen J, Gotz HM, Richardus JH, Hoebe CJ, Broer J, Coenen AJ, et al. Prevalence of urogenital Chlamydia trachomatis increases significantly with level of urbanisation and suggests targeted screening approaches: results from the first national population based study in the Netherlands. Sex Transm Infect. 2005;81(1):17-23.

85. van den Broek IV, van Bergen JE, Brouwers EE, Fennema JS, Gotz HM, Hoebe CJ, et al. Effectiveness of yearly, register based screening for chlamydia in the Netherlands: controlled trial with randomised stepped wedge implementation. BMJ. 2012;345:e4316.

86. van Aar F, de Moraes M, Morre SA, van Bergen JE, van der Klis FR, Land JA, et al. Chlamydia trachomatis $\lg G$ seroprevalence in the general population of the Netherlands in 1996 and in 2007: differential changes by gender and age. Sex Transm Infect. 2014;90(5):434-40.

87. Land JA, Van Bergen JE, Morre SA, Postma MJ. Epidemiology of Chlamydia trachomatis infection in women and the cost-effectiveness of screening. Hum Reprod Update. 2010;16(2):189-204.

88. Low N. Screening programmes for chlamydial infection: when will we ever learn? BMJ. 2007;334(7596):725-8.

89. van Valkengoed IG, Morre SA, van den Brule AJ, Meijer CJ, Bouter LM, Boeke AJ. Overestimation of complication rates in evaluations of Chlamydia trachomatis screening programmes-implications for cost-effectiveness analyses. Int J Epidemiol. 2004;33(2):416-25.

90. Herzog SA, Althaus CL, Heijne JC, Oakeshott $\mathrm{P}$, Kerry $\mathrm{S}$, Hay $\mathrm{P}$, et al. Timing of progression from Chlamydia trachomatis infection to pelvic inflammatory disease: a mathematical modelling study. BMC Infect Dis. 2012;12:187.

Herzog SA, Heijne JC, Althaus CL, Low N. Describing the progression from Chlamydia trachomatis and Neisseria gonorrhoeae to pelvic inflammatory disease: systematic review 
of mathematical modeling studies. Sex Transm Dis. 2012;39(8):628-37.

92. Price $\mathrm{MJ}$, Ades $\mathrm{AE}$, De Angelis $\mathrm{D}$, Welton NJ, Macleod J, Soldan K, et al. Risk of pelvic inflammatory disease following Chlamydia trachomatis infection: analysis of prospective studies with a multistate model. Am J Epidemiol. 2013;178(3):484-92

93. Kavanagh K, Wallace LA, Robertson C, Wilson $\mathrm{P}, \mathrm{Scoular}$ A. Estimation of the risk of tubal factor infertility associated with genital chlamydial infection in women: a statistical modelling study. Int J Epidemiol. 2013;42(2):493-503.

94. Andersen B, Ostergaard L, Puho E, Skriver MV, Schonheyder HC. Ectopic pregnancies and reproductive capacity after Chlamydia trachomatis positive and negative test results: a historical follow-up study. Sexually transmitted diseases. 2005;32(6):377-81.

95. Bakken IJ, Skjeldestad FE, Lydersen S, Nordbo SA. Births and ectopic pregnancies in a large cohort of women tested for Chlamydia trachomatis. Sex Transm Dis. 2007;34(10):73943.

96. ECDC. Chlamydia control in Europe Literature review ECDC. Stockholm: European Centre for Disease Prevention and Control; 2014.

97. van den Broek IV, Sfetcu O, van der Sande MA, Andersen B, Herrmann B, Ward $\mathrm{H}$, et al. Changes in chlamydia control activities in Europe between 2007 and 2012: a cross-national survey. Eur $\mathrm{J}$ Public Health. 2016;26(3):382-8.

98. Chandra NL, Soldan K, Dangerfield C, Sile B, Duffell S, Talebi A, et al. Filling in the gaps: estimating numbers of chlamydia tests and diagnoses by age group and sex before and during the implementation of the English National Screening Programme, 2000 to 2012. Euro Surveill. 2017;22(5)

99. Public Health England P. National Chlamydia Screening Programme (NCSP): programme overview London: PHE2003 [Available from: https://www.gov.uk/government/publications/ ncsp-programme-overview/ncsp-programme- overview.

100. Migchelsen SJ. Davis GS OK, Folkard K, Bleasdille M, Draper D, Shaw D, Saunders J, Buitendam E, Hollis E, Soldan K, Woodhall SC, Dunbar K, the NCSP team. National Chlamydia Screening Programme External Peer Review: evidence pack. London: Public Health England; 2017.

101. Low N, Redmond S, Uuskula A, van Bergen J, Ward $\mathrm{H}$, Andersen $\mathrm{B}$, et al. Screening for genital chlamydia infection. Cochrane Database Syst Rev. 2016;9:CD010866.

102. Hocking JS, Temple-Smith M, Guy R, Donovan B, Braat S, Law M, et al. Population effectiveness of opportunistic chlamydia testing in primary care in Australia: a cluster-randomised controlled trial. Lancet. 2018;392(10156):141322.

103. Oakeshott P, Kerry S, Aghaizu A, Atherton H, Hay S, Taylor-Robinson D, et al. Randomised controlled trial of screening for Chlamydia trachomatis to prevent pelvic inflammatory disease: the POPI (prevention of pelvic infection) trial. BMJ. 2010;340:c1642.

104. Scholes D, Stergachis A, Heidrich FE, Andrilla $\mathrm{H}$, Holmes KK, Stamm WE. Prevention of pelvic inflammatory disease by screening for cervical chlamydial infection. $N$ Engl $J$ Med. 1996;334(21):1362-6.

105. Kabisch M, Ruckes C, Seibert-Grafe M, Blettner M. Randomized controlled trials: part 17 of a series on evaluation of scientific publications. Dtsch Arztebl Int. 2011;108(39):663-8.

106. Thygesen LC, Ersboll AK. When the entire population is the sample: strengths and limitations in register-based epidemiology. Eur J Epidemiol. 2014;29(8):551-8.

107. Price MJ, Horner PJ, Ades AE. Risk of reproductive complications following chlamydia testing. Lancet Infect Dis. 2016;16(11):1223-4.

108. Chow JM, Yonekura ML, Richwald GA, Greenland S, Sweet RL, Schachter J. The association between Chlamydia trachomatis and ectopic pregnancy. A matched-pair, casecontrol study. JAMA. 1990;263(23):3164-7. 
109. Rashidi BH, Chamani-Tabriz L, Haghollahi F, Jeddi-Tehrani M, Naghizadeh MM, Shariat M, et al. Effects of Chlamydia trachomatis Infection on Fertility; A Case-Control Study. J Reprod Infertil. 2013;14(2):67-72.

110. Akande VA, Hunt LP, Cahill DJ, Caul EO, Ford WC, Jenkins JM. Tubal damage in infertile women: prediction using chlamydia serology. Hum Reprod. 2003;18(9):1841-7.

111. Tenny S, Hoffman MR. Case Control Studies. StatPearls. Treasure Island (FL)2020.

112. Beyuo T, Oppong SA, Samba A, Beyuo VM. Chlamydia trachomatis infection among Ghanaian women undergoing hysterosalpingography for suspected tubal factor infertility. Int $J$ Gynaecol Obstet. 2019;146(2):200-5

113. Fernandes LB, Arruda JT, Approbato MS, Garcia-Zapata MT. [Chlamydia trachomatis and Neisseria gonorrhoeae infection: factors associated with infertility in women treated at a human reproduction public service]. Rev Bras Ginecol Obstet. 2014;36(8):353-8.

114. Mann CJ. Observational research methods. Research design II: cohort, cross sectional, and case-control studies. Emerg Med J. 2003;20(1):54-60.

115. Price MJ, Ades AE, Welton NJ, Macleod J, Turner K, Simms I, et al. How much tubal factor infertility is caused by Chlamydia? Estimates based on serological evidence corrected for sensitivity and specificity. Sex Transm Dis. 2012;39(8):608-13

116. Herzog SA, Low N, Berghold A. Sample size considerations using mathematical models: an example with Chlamydia trachomatis infection and its sequelae pelvic inflammatory disease. BMC Infect Dis. 2015;15:233.

117. Omori R, Chemaitelly $H$, Althaus CL, AbuRaddad LJ. Does infection with Chlamydia trachomatis induce long-lasting partial immunity? Insights from mathematical modelling. Sex Transm Infect. 2019;95(2):11521.

118. Murad MH, Asi N, Alsawas M, Alahdab F.
New evidence pyramid. Evid Based Med. 2016;21(4):125-7.

119. Haggerty CL, Gottlieb SL, Taylor BD, Low N, Xu F, Ness RB. Risk of sequelae after Chlamydia trachomatis genital infection in women. J Infect Dis. 2010;201 Suppl 2:S134-55.

120. Unemo M, Bradshaw CS, Hocking JS, de Vries HJC, Francis SC, Mabey D, et al. Sexually transmitted infections: challenges ahead. Lancet Infect Dis. 2017;17(8):e235-e79.

121. Euser AM, Zoccali C, Jager KJ, Dekker FW. Cohort studies: prospective versus retrospective. Nephron Clin Pract. 2009;113(3):c214-7.

122. Thiese MS. Observational and interventional study design types; an overview. Biochem Med (Zagreb). 2014;24(2):199-210.

123. Cohort Studies in Medical Research. InTechOpen; 2018. p. 11-28.

124. Grimes DA, Schulz KF. Cohort studies: marching towards outcomes. Lancet. 2002;359(9303):341-5.

125. Munnangi S, Boktor SW. Epidemiology Of Study Design. StatPearls. Treasure Island (FL)2020.

126. Grimes DA, Schulz KF. Bias and causal associations in observational research. Lancet. 2002;359(9302):248-52.

127. Levin KA. Study design IV. Cohort studies. Evid Based Dent. 2006;7(2):51-2.

128. Gamble JM. An introduction to the fundamentals of cohort and case-control studies. Can J Hosp Pharm. 2014;67(5):366-72.

129. Song JW, Chung KC. Observational studies: cohort and case-control studies. Plast Reconstr Surg. 2010;126(6):2234-42. 



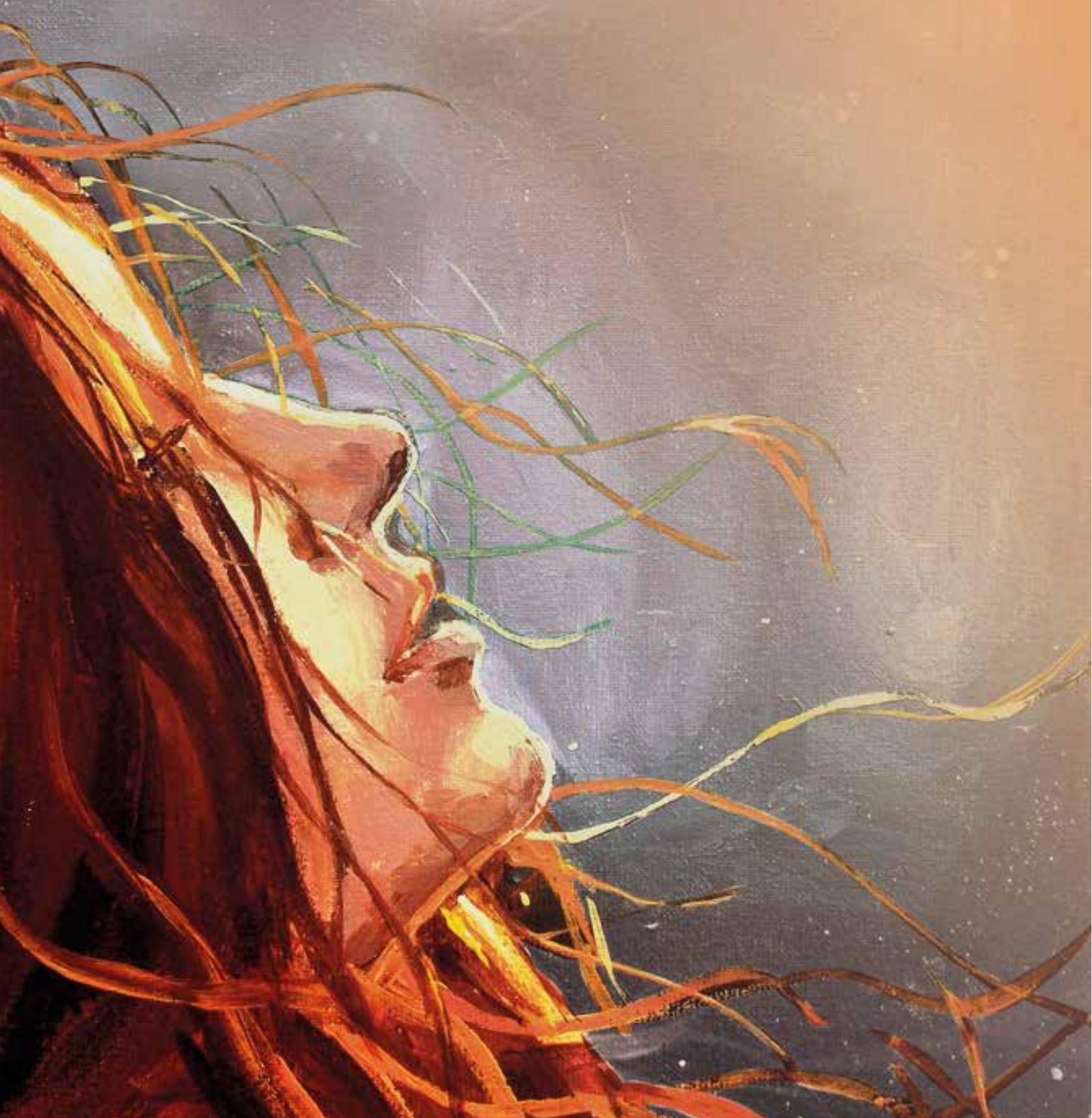




\section{Part 1}

The risk of complications following chlamydia

\section{infection: Setting up a cohort study}




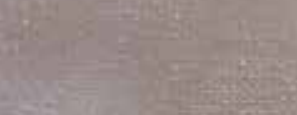

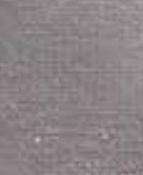

\section{sisting}

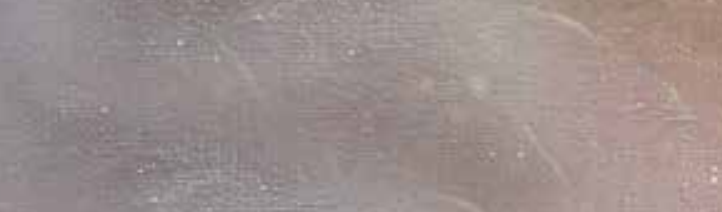

जince

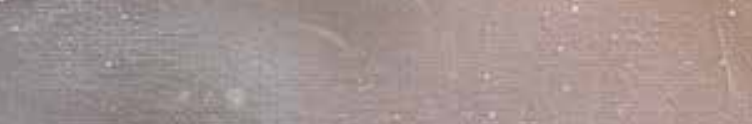

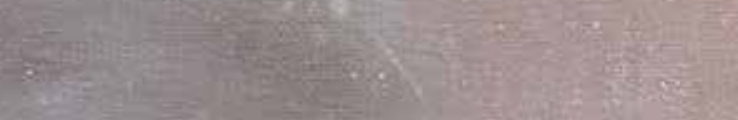

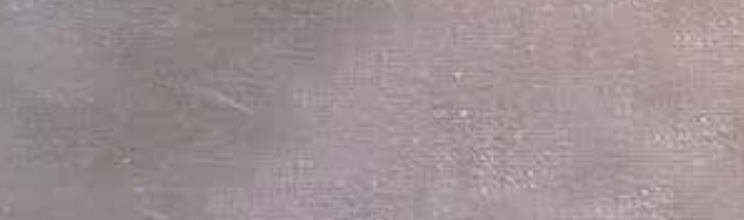

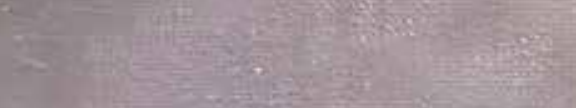

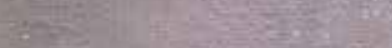

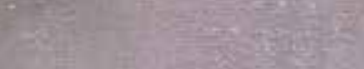

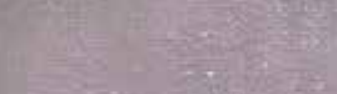

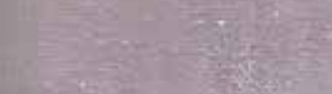

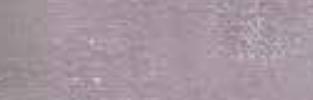

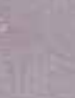

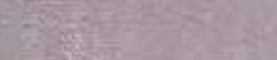

netwo

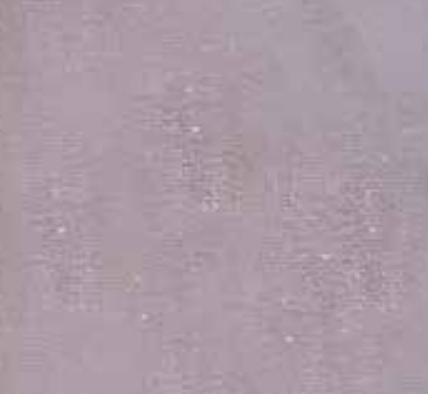

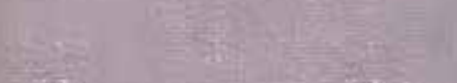

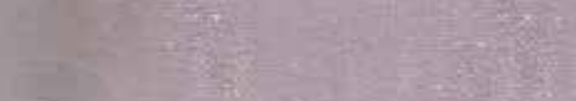




\section{2}

\section{The Netherlands Chlamydia Cohort Study}

\section{(NECCST) protocol to assess the risk of late}

complications following Chlamydia trachomatis infection in women

Bernice M Hoenderboom, Louise A van Oeffelen, Birgit HB van Benthem, Jan EAM van Bergen, Nicole HTM Dukers-Muijrer, Hannelore M Götz, Christian JPA Hoebe, Arjan A Hogewoning, Fiona RM van der Klis, Debbie van Baarle, Jolande A Land, Marianne AB van der Sande, Maaike G van Veen, Frank de Vries, Servaas A Morré, Ingrid VD van den Broek 


\section{Abstract}

Background: Chlamydia trachomatis (CT), the most common bacterial sexually transmitted infection (STI) among young women, can result in serious sequelae. Although the course of infection is often asymptomatic, CT may cause pelvic inflammatory disease (PID), leading to severe complications, such as prolonged time to pregnancy, ectopic pregnancy, and tubal factor subfertility. The risk of and risk factors for complications following CT-infection have not been assessed in a long-term prospective cohort study, the preferred design to define infections and complications adequately.

Methods: In the Netherlands Chlamydia Cohort Study (NECCST), a cohort of women of reproductive age with and without a history of CT-infection is followed over a minimum of ten years to investigate (CT-related) reproductive tract complications. This study is a follow-up of the Chlamydia Screening Implementation (CSI) study, executed between 2008 and 2011 in the Netherlands. For NECCST, female CSI participants who consented to be approached for followup studies $(n=14,685)$ are invited, and prospectively followed until 2022. Four data collection moments are foreseen every two consecutive years. Questionnaire data and blood samples for CT-Immunoglobulin G ( $\mathrm{IgG}$ ) measurement are obtained as well as host DNA to determine specific genetic biomarkers related to susceptibility and severity of infection. CT-history will be based on CSI test outcomes, self-reported infections and CT-IgG presence. Information on (time to) pregnancies and the potential long-term complications (i.e. PID, ectopic pregnancy and (tubal factor) subfertility), will be acquired by questionnaires. Reported subfertility will be verified in medical registers. Occurrence of these late complications and prolonged time to pregnancy, as a proxy for reduced fertility due to a previous CT-infection, or other risk factors, will be investigated using longitudinal statistical procedures.

Discussion: In the proposed study, the occurrence of late complications following CT-infection and its risk factors will be assessed. Ultimately, provided reliable risk factors and/or markers can be identified for such late complications. This will contribute to the development of a prognostic tool to estimate the risk of CT-related complications at an early time point, enabling targeted prevention and care towards women at risk for late complications. 


\section{Background}

Chlamydia trachomatis (CT) is the most commonly reported bacterial sexually transmitted infection (STI) in the Netherlands [1]. In contrast to most other STIS, CT is prevalent in a large segment of the population [2]. In the Netherlands, the reporting rate of CT-infections has steadily increased from 2.7/1,000 persons in 2010 to 3.1/1,000 in 2014, based on data from STI clinics and general practitioners (GPs). This is mainly due to increased testing rates in high-risk groups [1]. Enhanced testing in the Netherlands - as performed in the population-based Chlamydia Screening Implementation (CSI) study with annual screening between 2008 and 2011 - did not demonstrate a (cost-) effective reduction of CT prevalence, related to the relatively low and declining participation rates in the trial [3-5]. Observational studies in other European countries and a large CT screening pilot via general practitioners in Australia, showed similar results [6, 7]. In addition, in countries that have active screening policies (e.g. UK and USA) the number of CT-diagnoses in the population targeted for routine annual screening did not decline $[6,8]$. New strategies for CT control are urgently needed.

Women bear a disproportional burden of CT-infections [9]. In the Netherlands, prevalence of CT around 2010 was estimated at 2.9\% among 16-25 year-old women [5]. Since CT-infections in women have an asymptomatic course in up to $70 \%$ of the cases, most of these women will remain untreated $[10,11]$. Meanwhile the CT-infection can ascend to the upper genital tract, resulting in pelvic inflammatory disease (PID), potentially causing tubal damage. Tubal damage in its turn may lead to ectopic pregnancy and tubal factor subfertility [12]. These complications only become apparent when women try to become pregnant, often several years after an initial CT-infection that may have gone unnoticed. A prior CT-infection may also prolong time to pregnancy in women without any visible tubal pathology, due to damage to the tubal mucosa (compromising embryo transport) or unfavourable effects in the endometrium (affecting implantation) [13].

The proportion of women experiencing CT-complications is largely unknown due to the asymptomatic nature of the infection, delayed awareness of the actual pathology, and the long follow-up period needed before complications become apparent [14]. Current risk estimates range widely; the estimated risk of PID following a CT-infection ranges between $0.5 \%$ and $72 \%$ depending on the study population and the definitions used [15-20]. Subfertility as a result of prior CT-infection is estimated to occur in $0.1-6 \%$ of women infected $[15,21]$, and $0-1 \%$ of women with a prior infection may develop an ectopic pregnancy [22, 23]. In a large retrospective population-based cohort of 500,000 women aged 15-44 years in Denmark, the risk of PID, ectopic pregnancy and tubal factor subfertility was found to be at least $30 \%$ higher in women who had tested CT-positive in the past compared to women who had only negative tests. In addition, repeated diagnoses of CT-infections increased the risk of PID by 22\% [24]. Recent estimates by Price et al in the UK, based on results of major studies and study designs, suggest that $20 \%$ of PIDs, $5 \%$ of ectopic pregnancies, and $30 \%$ of tubal factor subfertility cases are attributable to CT women aged 16-44 years [25].

Rather than attempting to trace and treat all CT-infections, it might be more effective to pursue secondary prevention in women at higher risk for complications. Host genetic biomarkers are 
considered to play a role in the development of complications in the reproductive tract after CTinfection [26]. In Gambian twin pairs, Bailey et al. found that $40 \%$ of the host response to trachoma (i.e. a tropical eye infection caused by CT serovar A or B) is linked to host genetic characteristics [27]. The scarring of the eye and the scarring of the tubes have a remarkable immunogenetic resemblance [26], and therefore we hypothesize a similar effect of host genetics in CT-related trachoma and tubal pathology. Subsequently, the presence of Single Nucleotide Polymorphisms (SNPs) in genes, identified by candidate gene studies and Genome Wide Association Studies (GWAS), has already been shown to be related to the development of tubal pathology following CT-infection. For example, carriage of two or more SNPs in toll-like receptor (TLR)9, TLR4, cluster of differentiation (CD) 14 and caspase recruitment domain (CARD)15/nucleotide oligomerisation domain (NOD)2 increased the risk of tubal pathology following CT-infection from 33\% to $73 \%$, though the increase was not statistically significant [28]. Furthermore, carriership of mannose binding lectin (MBL) Codon 54 allele B was higher among CT-positives with tubal pathology (OR $3.9,95 \% \mathrm{Cl} 1.9-8.2)[29]$ than among CT-positive controls and carriage of the NOD1 +32656 GG insertion was more frequent in women with TFI compared to women without TFI (OR 2.3, 95\% CI 1.1-4.7) [30].

A range of other (host) factors such as clinical symptoms, co-infections, re-infections and sexual risk behaviour may influence the development of complications in women with a previous CTinfection [24, 31-33]. Some factors related to the (severity of) infection or sexual behavioural are time-dependent. To precisely quantify the risk and predisposing risk factors of PID, ectopic pregnancy and tubal factor subfertility following a CT-infection, prospective studies are needed to provide vital data for programs to prevent CT-infection and complications [14]. Therefore, the NEtherlands Chlamydia Cohort STudy (NECCST) was initiated. The strength of the study is its prospective design because this enables to collect, at regular time intervals, questionnairedata with minimum (recall) bias, facilitates more extensive time analysis and allows to directly asses the risk of CT-related complications. The final aim will be to identify women most at risk for developing complications, in order to introduce targeted preventive measures and strategies to prevent CT (re-)infections in women at high risk for complications. Here we describe the NECCST cohort study design.

\section{Study aim}

With NECCST, we aim to gain more insight in late complications of CT-infections in women, and to identify women most at risk to develop CT-related complications. In this cohort study, the following aims will be addressed:

Primary objective:

- To quantify the incidence of PID, ectopic pregnancy and tubal factor subfertility and compare time to pregnancy in women with and without a previous CT-infection, in order to estimate the inherent risk of these outcomes by a previous CT-infection.

Secondary objectives:

- To explore which combination of host genetic biomarkers are able to distinguish women with a high risk of developing CT-related complications from women with a low risk of 
developing these complications.

- To determine demographic, behavioural, serological and infection-related factors that are associated with reproductive tract complications due to a preceding CT-infection.

\section{Methods}

\section{Design}

NECCST is a long-term (10-14 years) cohort study, of women of reproductive age who previously participated in the CSI study.

\section{Setting}

The starting point of the NECCST cohort is retrospective, at entry in the CSI study conducted between 2008 and 2011 in Amsterdam, Rotterdam and South Limburg (the Netherlands). In CSI, over 420,000 sexually active young adults (16-29 years old) who were registered in the municipal population registers of three areas (Amsterdam, Rotterdam and South-Limburg) were invited for annual CT-testing by home-based self-collection of a vaginal swab or urine sample. The samples were tested for a CT-infection using nucleic acid amplification tests (NAAT). In addition, participants completed questionnaires concerning demographic factors, sexual behaviour and previous STIs. Altogether, 80,000 people (19\% of those invited) participated in CSI. Compared to non-responders, CSI participants were more often women, older (20-29 years), had a higher education, and were more often of a Dutch background. For the NECCST cohort, invitees were further selected from this group [4].

\section{Study population}

All women who participated in at least one round of the CSI study ( $n=58,818,26 \%$ of those invited) and who gave informed consent to be approached for future STI-related research $(14,685,25 \%)$, are invited for participation in NECCST. Of the eligible women, 2,371 (16\%) had a positive CThistory (i.e. PCR positive result and/or self-reported CT-infection during CSI) and 12,314 (84\%) had a negative CT-history according to CSI data (PCR negative and no self-reported previous CT-infection). These women are traced in the Dutch municipal registers and invited for participation in NECCST. Participants are between 20 and 38 years of age at the first data collection moment, and will be between 27 and 44 years of age by the end of NECCST. Women are censored when they have emigrated from the Netherlands or when contact details cannot be retrieved from the municipal registers for other reasons.

\section{Study procedures}

NECCST covers a minimal individual follow-up period of 10 years and a maximum of 14 years, depending on inclusion in CSI, onwards. Participants will be contacted four times until 2022.

In 2015/2016, women were informed by regular mail and email (when available from CSI) about NECCST, and received an invitation letter and an information brochure. Via a web link in the invitation letter women could decline or accept participation. After one month a reminder is sent. 
After accepting, participating women were requested to complete an online informed consent form for participation in NECCST.

The first data collection moment includes an electronic questionnaire followed by a test kit for self-collection of blood by a finger-prick for CT IgG analyses. Host DNA is obtained by either using the stored CSI vaginal swabs or urine, or via additional newly self-collected buccal swabs [Copan FLOQSwabsTM, Copan diagnostics, USA]. Every two years, an online questionnaire will be sent by email. In 2022, at the last data collection moment, a second test kit for self-collection of blood will be sent for CT IgG analyses (Figure 1, study flowchart).

\section{Data collection during NECCST}

Questionnaires

At the start of the NECCST follow-up period, women were asked to complete a baseline questionnaire. Thereafter, they receive a follow-up online questionnaire once every two years. The questionnaires inquire data about recent and past CT-infections and CT-related PID, time to pregnancy, ectopic pregnancy, and fertility problems. In addition, questionnaires will address demographic factors, age, ethnicity, educational level, sexual behaviour, other STIs, contraceptive use and health characteristics (e.g. smoking, weight changes, chronic pelvic pain (abdominal pain with a duration of 6 months or more) and previous abdominal surgery). Questionnaires are all electronic, sent with Formdesk [Formdesk, Wassenaar, The Netherlands], which creates a database automatically, linkable to other data sources (i.e. CSI-database), by individual participant number.

\section{Medical register check}

All women who report subfertility are asked additional informed consent to allow us to approach their GPs and/or fertility clinics to provide specified, detailed information regarding the cause of subfertility from the patient's files in medical registers. 


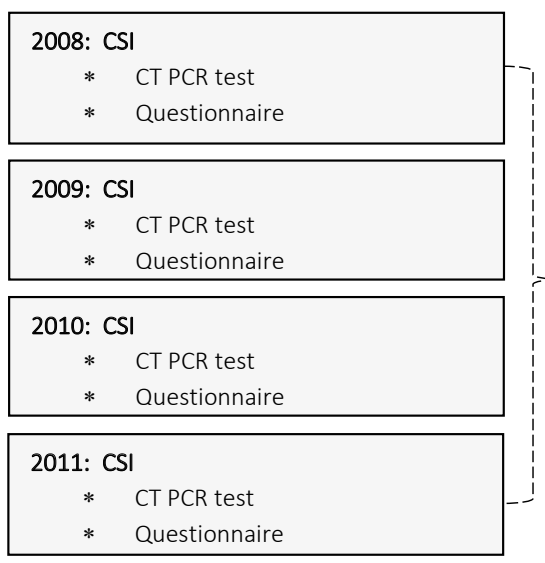

$C S I$

$\downarrow \quad 228,457$ women were invited

$\downarrow \quad 58,818$ (26\%) women were tested at least once

$\downarrow \quad 14,685$ (6\%) women gave informed consent to be contacted for future research

\section{5-2016: NECCST}

First round:

* Questionnaires

* Medical register check*

* CT IgG analyses

* Retrieving biobank samples

2017-2018: Second round:

* Questionnaires

* Medical register check*

\section{9-2020: Third round:}

* Questionnaires

* Medical register check*

\section{1-2022: Last round:}

* Questionnaires

* Medical register check*

* CT IgG analyses
Start NECCST

14,685 (100\%) women will be traced and invited for NECCST

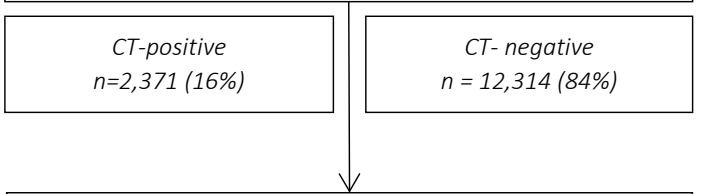

During NECCST we expect:

$40 \%$ non-response

$15 \%$ attrition

12\% cross over from Ct negative to CT-positive

\section{End NECCST}

6,600 (45\%) women expected to complete follow-up

\begin{tabular}{|c|c|}
\hline CT-positive \\
$n=1,700(26 \%)$
\end{tabular}$\quad \begin{gathered}\text { CT-negative } \\
n=4,900(74 \%)\end{gathered}$

Figure 1. Flowchart of the study. * In case subfertility is reported, the participants' medical files will be requested to verify the cause of subfertility. CSI $=$ Chlamydia Screening Implementation. NECCST = Netherlands Chlamydia Cohort Study. CT = Chlamydia trachomatis. PCR $=$ Polymerase chain reaction. IgG = Immunoglobulin G. CT-positive = at least one positive outcome, either a positive CSI CT Polymerase Chain Reaction (PCR) result, a self-reported CT-infection or CT IgG presence. CT-negative $=$ Never tested positive during CSI, no self-reported CT-infection and no CT IgG presence.

\section{Chlamydia trachomatis serology}

A test kit will be sent to participants to collect a capillary blood sample at home in a collection tube [BD Microtainer serum separator tube, Becton, Dickinson and Company, USA] and to return it to the laboratory in the accompanying packaging. All returned samples are immediately processed and blood collection tubes are centrifuged to collect serum. Serum samples are stored at $-20^{\circ} \mathrm{C}$ 
until an ELISA assay is performed. CT IgG antibodies are measured using a peptide based ELISA test [Medac CT IgG pELISA, Wedel, Germany] with minimal cross-reactivity and high throughput [34]. CT IgG antibody test results will be used as a marker for a previous CT-infection which remained unnoticed [35]. In addition, the seroconversion rate will be analysed in women with a previously self-reported or PCR confirmed CT-infection. An additional IgG antibody test will be performed in 2022, in order to determine new infections occurring during the study period and to gain insight in persistence of CT IgG levels over time.

\section{Host genetic biomarkers}

Host genetic biomarkers (SNPS), will be determined from host material obtained from vaginal swabs and urine samples collected during the CSI study and stored in a biobank. DNA will be extracted and host genetic biomarkers will be analysed using Kompetitive Allele Specific PCR (KASP) technology, utilizing a unique form of competitive allele-specific polymerase chain reaction (PCR). This enables accurate scoring of SNPs, inserts or deletions [36]. A selection of 50-100 SNPs will be tested. This SNP panel is based on previous research, that showed a potential association with chlamydia susceptibility and risk to develop complications after infection [26]. From participants whose previous CSI study sample is not available or of insufficient quality, a buccal swab sample is obtained at the start of NECCST in order to obtain DNA.

\section{Defining CT-history}

As CT-infections often go unnoticed it was decided to define a positive CT-history based on one of the following three outcomes, either a self-reported CT-infection, positive PCR-test outcome in the CSI study or the presence of CT IgG antibodies in serum. Combining these three outcomes will reduce misclassification of CT-history.

\section{Power calculation}

In total 14,685 women are invited of whom 2,371 had a positive CT-history and 12,314 had a negative CT-history recorded in the CSI study. Assuming a high response rate of $60 \%$ (due to previous informed consent), a cross-over rate of $12 \%$ from negative to positive CT-history and an estimated loss to follow up of $25 \%$ until 2022, we expect to have 1,700 women with a positive CT-history and 4,900 with a negative CT-history participating in NECCST until the end of the study in 2022. Power calculations, based on the primary aim were performed using risk estimates from modelling and observational studies. In women with a positive CT-history the following risk estimates were taken into account: 10\% for developing PID, 0.5\% for ectopic pregnancy and $2 \%$ for tubal factor subfertility $[15,22,23]$. In women with a negative CT-history, the risk to develop these complications was estimated to be $0.5 \%$ for PID [37], and $0.05 \%$ for tubal factor subfertility [15]. For ectopic pregnancy, the expected risk ranges between 0 and $1 \%$ in CT negatives [22, 23]. Expected cumulative prevalence's of these complicates were calculated based on age at the end of NECCST in 2022 (Table 1). The expected sample size of the study population, i.e. 1,700 women with a positive CT-history and 4,900 women with a negative CT-history, is sufficient to detect significant differences $(\mathrm{p}<0.05)$ in risks of CT-related complications between women with and without a positive CT-history with a power of $85 \%-99 \%$. 
Table 1. Power calculation per outcome variable

\begin{tabular}{llllllr}
\hline & $\begin{array}{l}\text { Expected } \\
\text { cumulative } \\
\text { prevalence in 2022 }\end{array}$ & $\begin{array}{l}\text { Number of } \\
\text { samples needed } \\
\text { for 80\% power }\end{array}$ & $\begin{array}{l}\text { Expected power with } \\
\text { samples size N = 6,600 }\end{array}$ \\
\hline & $\begin{array}{l}\text { CT pos. } \\
\text { history }\end{array}$ & $\begin{array}{l}\text { CT neg. } \\
\text { history }\end{array}$ & $\begin{array}{l}\text { CT pos. } \\
\text { history }\end{array}$ & $\begin{array}{l}\text { CT neg. } \\
\text { history }\end{array}$ & $\begin{array}{l}\text { CT pos. } \\
\text { history } \\
(\mathrm{n}=1,700)\end{array}$ & $\begin{array}{l}\text { CT neg. } \\
\text { history } \\
(\mathrm{n}=4,900)\end{array}$ \\
PID & $7.7 \%$ & $1.1 \%$ & 63 & 195 & & $>99 \%$ \\
Ectopic pregnancy & $1.8 \%$ & $0.9 \%$ & 1,415 & 4,385 & $85 \%$ \\
Tubal factor subfertility & $1.3 \%$ & $0.2 \%$ & 401 & 1,241 & & $>99 \%$ \\
\hline
\end{tabular}

Expected cumulative prevalence of PID, ectopic pregnancy and tubal factor subfertility was based on age distribution per outcome in the primary care database from the Netherlands Institute for Health Services Research (NIVEL - PCD) and the expected age distribution in NECCST in 2022 by CT status. Here from we derived the samples size, with $80 \%$ power, a significance level of $5 \%$ and a 1 to 3 exposed/unexposed ratio, per outcome in the CT-positive history and negative history group in 2022 (www.openepi.com). CT = Chlamydia trachomatis infection. PID = pelvic inflammatory disease.

\section{Data analysis and statistics}

Data from the CSI-study will be merged with data from NECCST. Data quality will be assessed, in particular the potential for bias due to non-response and the extent of missing data. Incidence rates, calculated as the number of new cases divided by the total person-time at risk, of the primary study endpoints PID, ectopic pregnancies and tubal factor subfertility will be compared between women with and without a positive CT-history. Person-time at risk is calculated from the time point a woman becomes sexually active (assessed from the CSI questionnaire) and ends at the time of an event. In case of no event, person-time at risk stops at the end of participation, migration out of the Netherlands or the end of the study period, whichever comes first.

Each of the primary study endpoints PID, ectopic pregnancy and tubal factor subfertility will be analysed for women with and without a positive CT-history using Kaplan-Meier plots and log-rank test. Cox proportional hazards regression analyses will be performed to calculated hazard ratios, in which CT-history is included as a time-dependent variable. Once a participant becomes CTpositive, the participant switches over to the CT-positive group. We will explore if time to infection for women with a positive CT-history based only on their CT IgG presence without further information on the time of infection, can be estimated using information from the group with both a positive IgG test result and a positive PCR test or self-reported CT-infection. To account for confounding and effect modification, co-factors such as age, educational level, host genetic biomarkers, demographics, behavioural and infection characteristics (i.e. as previous treatment and other STI's), will be included in the model.

Similar analyses will be done to assess the factors related to time to pregnancy. For time to pregnancy the follow-up period is defined as the time in months between the moment the woman reports starting to try to conceive until the start of pregnancy, or (if not pregnant) the date of completion of the questionnaire.

Univariate and multivariate Cox regression analyses will also be applied to investigate covariates among women with a positive CT-history and the event of a PID, ectopic pregnancy or tubal 
factor subfertility. All factors that are significantly associated with the specific complication will be included in a multivariate Cox proportional hazards regression model. Using backward stepwise selection, factors that are not associated with the development of the complication anymore (with a threshold p-value of 0.1 ) will be removed. All other factors will be assigned significantly associated with the development of the complication. The following literature-based variables will be selected as a potential predictor: age, educational level, host genetic biomarkers, and demographic, behavioural and infection characteristics.

Sensitivity analyses will be performed by varying the definitions of a positive CT-history, i.e. only CSI-PCR positive outcomes compared to self-reported CT-infections and CT IgG positivity. We will also perform sensitivity analyses on variable definition of outcome variables, such as planned versus unplanned pregnancies and confirmed versus unconfirmed tubal factor subfertility.

This study was approved by the Medical Ethical Committee Noord-Holland, Alkmaar the Netherlands (NL 51553.094.14/M014-042). All participants provide informed consent for participation in NECCST.

\section{Discussion}

In NECCST, the risk of PID, time to pregnancy, tubal factor subfertility and ectopic pregnancy after CT-infection will be determined in a cohort of women of reproductive age with an individual follow-up time of 10 to 14 years. NECCST will investigate the role of a wide range of host- and infection-specific factors in the development of CT-related complications. Insight in risk factors of CT-related complications may allow for a new strategy in prevention of the complications of CT. This could be an alternative approach in addition to current chlamydia control strategies, aiming to test and treat to prevent ongoing transmission. The ultimate goal will be to develop a prognostic tool to identify the group of women with an enhanced risk of complications after a CT-infection at an early time point in their life, when (secondary) preventive measures can be effectively applied. Women at high risk for complications could be specifically targeted for prevention of (re-)infection, e.g. by frequent follow-up as an optimal strategy for preventing long-term complications [24].

\section{Strengths}

NECCST will be the first cohort study in which risks of and for late complications of CT are prospectively studied during a follow-up period of more than 10 years. A prospective study design allows for a clear temporal sequence of exposure and outcome and examination of multiple effects of a single exposure while avoiding selection bias at enrolment. The long-term follow-up is needed to examine the relation between exposure and outcome, as CT-infections are often acquired below the age of 20 , fertility problems will only become apparent when a woman tries to become pregnant, which is often in her late twenties or thirties.

This cohort combines several data sources: historical data from the CSI, newly acquired questionnaire data at four time points during NECCST, medical register data, serological outcomes and host genetic biomarkers. By combining information from tests from the CSI study, self-reported CT-infections and CT IgG status, we expect to obtain a more complete picture of previous CT- 
infections than in most other studies. As we make use of serology, we will also be able to identify women who had a CT-infection which remained unnoticed and was therefore left untreated. These women can be compared to women who tested positive for CT and were treated accordingly. Comparing these groups may give us more insight into the lgG status and risk of CT-related complications between treated and untreated women and allow us to study the natural course of a CT-infection [14]. Host genetic biomarkers are nowadays implemented in prediction models and health care systems. Developing a set of specific genetic biomarkers associated with a high risk for CT-related complications can facilitate identifying the group of women most vulnerable for developing complications [26, 38]. With a 'precision (or personalized) medicine approach' a diagnostic tool on the basis of women's genetic profile can be employed for selecting the group to be targeted for specific (cost)effective interventions such as repeated CT-testing, additional treatment and medical follow-up.

\section{Limitations}

Although in our study CT-history will be based on PCR results from the CSI study, self-reported infections and CT IgG measurements, misclassifications may still occur. Women with an unnoticed infection may have a negative CT IgG antibody test, because an infection not always leads to CT IgG antibody production, or because the levels of antibodies have waned since the time of infection. The proportion of infected women who seroconvert and who remain persistently seropositive is not well established yet [35]. Sensitivity and specificity of CT IgG measurement of the assay we will use in NECCST (Medac pELISA) were reported to be $71.4 \%$ and $97.3 \%$ at the time of CT-infection, respectively [39]. However, within six months after infection, seropositivity was $66 \%$ and after six months or longer, seropositivity decreased to $38 \%$ [40], resulting in CT-positives possibly being misclassified as CT-negative. Because this could result in underestimation of the true association between CT-history and complications, we will perform sensitivity analyses in which only CSI-PCR positives will be classified as positive.

In our study, outcomes such as PID, tubal factor subfertility, ectopic pregnancy and time to pregnancy will be (initially) based on self-reporting, which may induce recall bias despite multiple questionnaire rounds every two years. The diagnosis of PID is imprecise and lacks a non-invasive accurate gold standard test [41]. Diagnostic bias could take place towards women with a positive CT-history [14]. This may result in a more pronounced underestimation of the risk on PID among CT-negatives than among CT-positives, potentially leading to an overestimation of the difference in PID risk between those groups. Therefore, in de questionnaire we specifically inquire about diagnosis by GP and hospital admissions for PID, and recall bias will be reduced. As a rough proxy for silent PID episodes, women are asked if they experienced chronic pelvic pain defined as unexplained abdominal pain with a duration of 6 months or more $[33,42]$. A range of other causes besides PID can result in chronic pelvic pain as well. However, assuming the incidences of these other causes to be evenly distributed between CT-positives and CT-negatives, any differences in the occurrence of chronic pelvic pain may be considered the result of PID. This will increase power and cover both symptomatic and silent PIDs.

In participants with an asymptomatic CT-infection we will not be able to determine when the first 
CT-infection and tubal scarring that eventually leads to subfertility have occurred. We cannot rule out that in some cases tubal damage was already present before the CT-infection. But in general tubal scarring may be assumed to occur after infection.

Finally, selection bias may occur when women who have experienced reproductive tract problems and have had a CT-infection are more willing to participate in NECCST. However, we expect that based on the relatively young age at the inclusion in NECCST, a substantial proportion of participants will not yet have tried to become pregnant when giving their consent to participate. Nevertheless, sensitivity analyses will be performed to compare outcomes in participants who were aware of reproductive complications at the start of NECCST with outcomes in those who were not yet aware.

\section{In summary}

In the future, instead of striving to detect and treat all CT-infections, chlamydia control strategies could focus on prevention of complications following a CT-infection. Long-term complications in women are the most important burden of CT-infections at population level. With this cohort study, we aim to contribute to better insight and further understanding of the factors involved in the development of late CT-complications, and to identify markers to understand which women are at risk of such complications. This should allow improved and targeted interventions to control adverse outcomes of CT infections. 


\section{References}

1. van den Broek IV, van Aar F, van Oeffelen AAM, Op de Coul EL, Woestenberg PJ, Heijne JC, den Daas C, Hofstraat SHI, Hoenderboom BM, van Wees $\mathrm{D}$ et al: Sexually transmitted infections in the Netherlands in 2015. In. Bilthoven, the Netherlands: National Institute for Public Health and the Environment; 2016, http://www.rivm.nl/ dsresource?objectid = e5493c29-6dc7-4372969b-9a55d36c88ca\&type $=$ org\&disposition $=7$. inline.

2. van Bergen J, Gotz H, Richardus JH, Hoebe C, Broer J, Coenen T, Group PCS: Prevalence of urogenital Chlamydia trachomatis infections in the Netherlands suggests selective screening 8 approaches. Results from the PILOT CT Population Study. Drugs of today 2006, 42 Suppl A:25-33, http://www.ncbi.nlm.nih.gov/ pubmed/16683041.

3. Schmid BV, Over EA, van den Broek IV, Op de Coul EL, van Bergen JE, Fennema JS, Gotz HM, Hoebe CJ, de Wit GA, van der Sande MA et al: Effects of population based screening for Chlamydia infections in the Netherlands limited by declining participation rates. PLoS One 2013, 8(3):e58674. 10.1371/journal.pone.0058674.

4. van den Broek IV, Hoebe CJ, van Bergen JE, Brouwers EE, de Feijter EM, Fennema JS, Gotz $H M$, Koekenbier RH, van Ravesteijn SM, de Coul EL: Evaluation design of a systematic, selective, internet-based, Chlamydia screening implementation in the Netherlands, 2008-2010: implications of first results for the analysis. $B M C$ Infect Dis 2010, 10:89. 10.1186/1471-2334-1089.

5. van den Broek IV, van Bergen JE, Brouwers EE, Fennema JS, Gotz HM, Hoebe CJ, Koekenbier $\mathrm{RH}$, Kretzschmar M, Over EA, Schmid BV et al: Effectiveness of yearly, register based screening for chlamydia in the Netherlands: controlled trial with randomised stepped wedge implementation. BMJ 2012, 345:e4316. 10.1136/bmj.e4316.

6. Sonnenberg P, Clifton S, Beddows S, Field
N, Soldan K, Tanton C, Mercer CH, da Silva FC, Alexander S, Copas AJ et al: Prevalence, risk factors, and uptake of interventions for sexually transmitted infections in Britain: findings from the National Surveys of Sexual Attitudes and Lifestyles (Natsal). Lancet 2013, 382(9907):1795-1806. 10.1016/S01406736(13)61947-9.

7. Hocking JS: Chlamydia control - where to from here? Results from the Australian Chlamydia control effectiveness pilot (ACCEPt). In: 8th meeting of the European Society of Chlamydia Research. Oxford, Town Hall; 2016.

8. Datta SD, Torrone E, Kruszon-Moran D, Berman S, Johnson R, Satterwhite CL, Papp $\mathrm{J}$, Weinstock H: Chlamydia trachomatis trends in the United States among persons 14 to 39 years of age, 1999-2008. Sex Transm Dis 2012, 39(2):92-96. 10.1097/OLQ.0b013e31823e2ff7.

9. Niccolai LM, Berube D: Burden of Sexually Transmitted Chlamydia trachomatis Infections. In: Handbook of Disease Burdens and Quality of Life Measures. Volume 1, edn. Edited by Preedy VR, Watson RR: Springer; 2010: 1297-1311.

10. Low N, McCarthy A, Macleod J, Salisbury C, Campbell R, Roberts TE, Horner P, Skidmore $\mathrm{S}$, Sterne JA, Sanford E et al: Epidemiological, social, diagnostic and economic evaluation of population screening for genital chlamydial infection. Health Technol Assess 2007, 11(8):iiiiv, ix-xii, 1-165, https://www.ncbi.nlm.nih.gov/ pubmed/17311735.

11. Farley TA, Cohen DA, Elkins W: Asymptomatic sexually transmitted diseases: the case for screening. Prev Med 2003, 36(4):502-509, https:// www.ncbi.nlm.nih.gov/pubmed/12649059.

12. ECDC: Chlamydia control in Europe Literature review ECDC. In. Stockholm: European Centre for Disease Prevention and Control; 2014.

13. Coppus SF, Land JA, Opmeer BC, Steures P, Eijkemans MJ, Hompes PG, Bossuyt PM, van der Veen F, Mol BW, van der Steeg JW: Chlamydia trachomatis IgG seropositivity 
is associated with lower natural conception rates in ovulatory subfertile women without visible tubal pathology. Hum Reprod 2011, 26(11):3061-3067. 10.1093/humrep/der307.

14. Haggerty CL, Gottlieb SL, Taylor BD, Low N, Xu $F$, Ness RB: Risk of sequelae after Chlamydia trachomatis genital infection in women. J Infect Dis 2010, 201 Suppl 2:S134-155. 10.1086/652395.

15. Land JA, Van Bergen JE, Morre SA, Postma $\mathrm{MJ}$ : Epidemiology of Chlamydia trachomatis infection in women and the cost-effectiveness of screening. Hum Reprod Update 2010, 16(2):189-204. 10.1093/humupd/dmp035.

16. Low $\mathrm{N}$ : Screening programmes for chlamydial infection: when will we ever learn? BMJ 2007, 334(7596):725-728. 10.1136/ bmj.39154.378079.BE.

17. van Valkengoed IG, Morre $S A$, van den Brule AJ, Meijer CJ, Bouter LM, Boeke AJ: Overestimation of complication rates in evaluations of Chlamydia trachomatis screening programmes--implications for costeffectiveness analyses. Int J Epidemiol 2004, 33(2):416-425. 10.1093/ije/dyh029.

18. Herzog SA, Althaus CL, Heijne JC, Oakeshott P, Kerry S, Hay P, Low N: Timing of progression from Chlamydia trachomatis infection to pelvic inflammatory disease: a mathematical modelling study. BMC infectious diseases 2012, 12:187. 10.1186/1471-2334-12-187.

19. Herzog SA, Heijne JC, Althaus CL, Low N: Describing the progression from Chlamydia trachomatis and Neisseria gonorrhoeae to pelvic inflammatory disease: systematic review of mathematical modeling studies. Sex Transm Dis 2012, 39(8):628-637. 10.1097/ OLQ.0b013e31825159ff.

20. Price MJ, Ades AE, De Angelis D, Welton 27. NJ, Macleod J, Soldan K, Simms I, Turner K, Horner PJ: Risk of pelvic inflammatory disease following Chlamydia trachomatis infection: analysis of prospective studies with a multistate model. Am J Epidemiol 2013, 178(3):484-492. 10.1093/aje/kws583.
21. Kavanagh K, Wallace LA, Robertson C, Wilson $\mathrm{P}$, Scoular A: Estimation of the risk of tubal factor infertility associated with genital chlamydial infection in women: a statistical modelling study. International journal of epidemiology 2013, 42(2):493-503. 10.1093/ije/dyt011.

22. Andersen B, Ostergaard L, Puho E, Skriver MV, Schonheyder HC: Ectopic pregnancies and reproductive capacity after Chlamydia trachomatis positive and negative test results: a historical follow-up study. Sexually transmitted diseases 2005, 32(6):377-381, http://www.ncbi. nIm.nih.gov/pubmed/15912085.

23. Bakken IJ, Skjeldestad FE, Lydersen S, Nordbo SA: Births and ectopic pregnancies in a large cohort of women tested for Chlamydia trachomatis. Sex Transm Dis 2007, 34(10):739743. 10.1097/01.olq.0000261326.65503.f6. https:// www.ncbi.nlm.nih.gov/pubmed/17479068

24. Davies B, Turner KM, Frolund M, Ward H, May MT, Rasmussen S, Benfield T, Westh H, Danish Chlamydia Study G: Risk of reproductive complications following chlamydia testing: a population-based retrospective cohort study in Denmark. Lancet Infect Dis 2016. 10.1016/ S1473-3099(16)30092-5.

25. Price MJ, Ades AE, Soldan K, Welton NJ, Macleod J, Simms I, DeAngelis D, Turner KM, Horner PJ: The natural history of Chlamydia trachomatis infection in women: a multiparameter evidence synthesis. Health Technol Assess 2016, 20(22):1-250. 10.3310/hta20220.

26. Morre SA, Karimi O, Ouburg S: Chlamydia trachomatis: identification of susceptibility markers for ocular and sexually transmitted infection by immunogenetics. FEMS Immunol Med Microbiol 2009, 55(2):140-153. 10.1111/j.1574-695X.2009.00536.X.

Bailey RL, Natividad-Sancho A, Fowler A, Peeling RW, Mabey DC, Whittle HC, Jepson AP: Host genetic contribution to the cellular immune response to Chlamydia trachomatis: Heritability estimate from a Gambian twin study. Drugs of today 2009, 45 Suppl B:45-50, http://www.ncbi. nIm.nih.gov/pubmed/20011694. 
28. den Hartog JE, Ouburg S, Land JA, Lyons JM, Ito JI, Pena AS, Morre SA: Do host genetic traits in the bacterial sensing system play a role in the development of Chlamydia trachomatis-associated tubal pathology in 36. subfertile women? BMC Infect Dis 2006, 6:122. 10.1186/1471-2334-6-122.

29. Sziller I, Babula O, Ujhazy A, Nagy B, Hupuczi 37 P, Papp Z, Linhares IM, Ledger WJ, Witkin SS: Chlamydia trachomatis infection, Fallopian tube damage and a mannose-binding lectin codon 54 gene polymorphism. Hum Reprod 2007, 22(7):1861-1865. 10.1093/humrep/dem107.

30. Brankovic I, van Ess EF, Noz MP, Wiericx WA Spaargaren J, Morre SA, Ouburg S: NOD1 in contrast to NOD2 functional polymorphism influence Chlamydia trachomatis infection and the risk of tubal factor infertility. Pathog Dis 2015, 73(1):1-9. 10.1093/femspd/ftu028.

31. Gotz HM, van den Broek IV, Hoebe CJ, Brouwers EE, Pars LL, Fennema JS, Koekenbier RH, van Ravesteijn S, Op de Coul EL, van Bergen J: High yield of reinfections by home-based automatic rescreening of Chlamydia positives in a largescale register-based screening programme and determinants of repeat infections. Sex Transm Infect 2013, 89(1):63-69. 10.1136/ sextrans-2011-050455.

32. Oakeshott P, Kerry $S$, Aghaizu A, Atherton H, Hay S, Taylor-Robinson D, Simms I, Hay $P$ : Randomised controlled trial of screening for Chlamydia trachomatis to prevent pelvic inflammatory disease: the POPI (prevention of pelvic infection) trial. BMJ 2010, 340:c1642. 10.1136/bmj.c1642.

33. Brunham RC, Gottlieb SL, Paavonen J: Pelvic inflammatory disease. N Engl J Med 2015, 372(21):2039-2048. 10.1056/NEJMra1411426.

34. Baud D, Regan L, Greub G: Comparison of five commercial serological tests for the detection of anti-Chlamydia trachomatis antibodies. Eur J Clin Microbiol Infect Dis 2010, 29(6):669-675. 10.1007/s10096-010-0912-4.

35. Johnson AM, Horner P: A new role for Chlamydia trachomatis serology? Sex Transm Infect 2008, 84(2):79-80. 10.1136/sti.2007.028472.

36. He C, Holme J, Anthony J: SNP genotyping: the KASP assay. Methods Mol Biol 2014, 1145:7586. 10.1007/978-1-4939-0446-4_7.

37. Dekker JHV, L.J.G.; Hinloopen, R.J.; van Kessel, T.; Boukes, F.S.: NHG-standaard Pelvic Inflammatory Disease (Eerste herziening). Huisarts Wet 2005, 48(10):509-513. https:// www.nhg.org/standaarden/samenvatting/ pelvic-inflammatory-disease

38. Asner SA, Morre SA, Bochud PY, Greub G: Host factors and genetic susceptibility to infections due to intracellular bacteria and fastidious organisms. Clin Microbiol Infect 2014, 20(12):1246-1253. 10.1111/1469-0691.12806.

39. Morre SA, Munk C, Persson K, Kruger-Kjaer $S$, van Dijk R, Meijer CJ, van Den Brule AJ: Comparison of three commercially available peptide-based immunoglobulin $\mathrm{G}(\mathrm{lg} G)$ and $\lg A$ assays to microimmunofluorescence assay for detection of Chlamydia trachomatis antibodies. J Clin Microbiol 2002, 40(2):584-587, http:// www.ncbi.nlm.nih.gov/pubmed/11825974

40. Horner PJ, Wills GS, Reynolds R, Johnson AM, Muir DA, Winston A, Broadbent AJ, Parker D, McClure MO: Effect of time since exposure to Chlamydia trachomatis on chlamydia antibody detection in women: a cross-sectional study. Sex Transm Infect 2013, 89(5):398-403. 10.1136/sextrans-2011-050386.

41. Simms I, Warburton F, Westrom L: Diagnosis of pelvic inflammatory disease: time for a rethink. Sex Transm Infect 2003, 79(6):491-494, http:// www.ncbi.nlm.nih.gov/pubmed/14663128

42. Wolner-Hanssen P: Silent pelvic inflammatory disease: is it overstated? Obstet Gynecol 1995, 86(3):321-325. 10.1016/0029-7844(95)00177$\mathrm{S}$. 


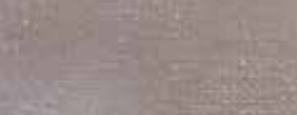

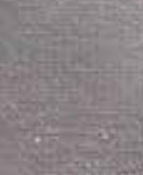

\section{sisting}

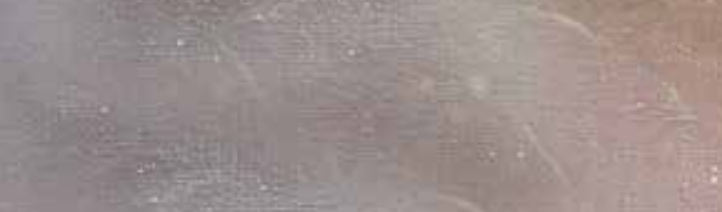

जince

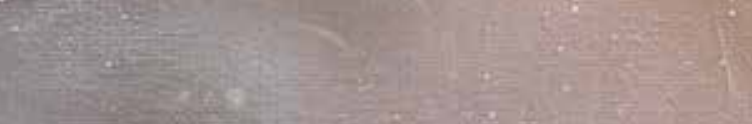

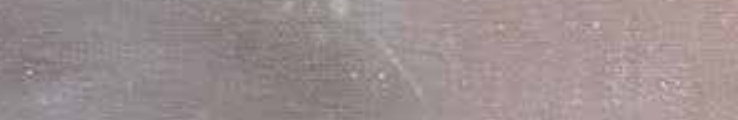

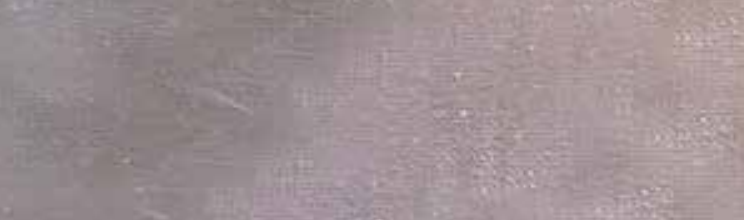

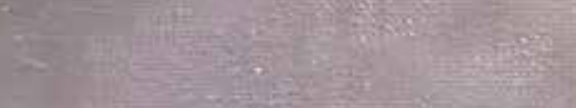

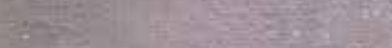

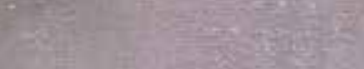

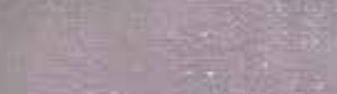

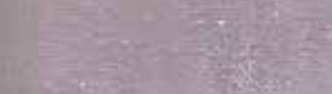

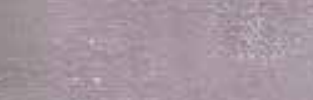

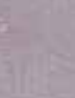

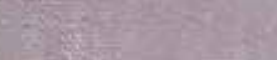

netwo

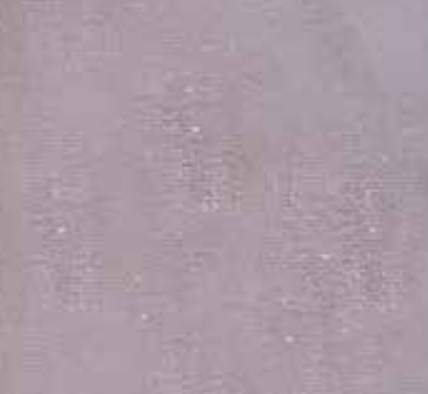

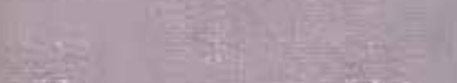

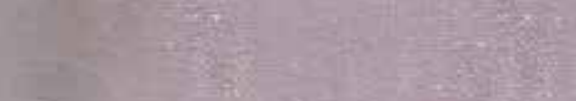




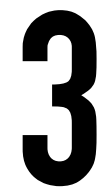

\section{Chlamydia trachomatis antibody detection in homecollected blood samples for use in epidemiological studies}

Bernice M Hoenderboom*, Eleanne F van Ess*, Ingrid VF van den Broek, Inge HM van Loo, Christian JPA Hoebe, Sander Ouburg, Servaas A Morré

*Shared first authorship

Published in: Journal of Microbiological Methods 2018, 10.1016/j.mimet.2017.11.022 


\section{Abstract}

Capillary blood collected in serum tubes was subjected to centrifugation delay while stored at room temperature. Chlamydia trachomatis (CT) IgG concentrations in aliquoted serum of these blood samples remained stable for seven days after collection. CT IgG concentrations can reliably be measured in mailed blood samples in epidemiological studies. 


\section{Introduction}

Implementing Chlamydia trachomatis (CT) antibody testing in large cohort studies increases insight in CT infection history. CT-infections are often missed due to the asymptomatic nature of the infection (1-3), however 40-100\% of women test positive for CT antibodies after CT-infection (4-7). Using antibody testing increases insight in CT infection history compared to only using selfreported CT infection history or test for prevalent infection (8). Therefore, the Netherlands Chlamydia Cohort Study (NECCST), a large cohort study determining the risk of CT related complications, uses antibody testing to determine CT infection history (9). To test for CT antibodies in NECCST a low cost, practical and reliable blood collection method was required (10).

The most practical collection method is capillary blood drawing by participants at home. Collected blood can be sent back to the laboratory by regular mail $(9,11)$. Blood can either be collected on filter paper or in capillary blood collection tubes with serum separator additive. Previously CT IgG was tested for validation using dried blood spots (DBS) versus clinician derived regular serum collected for a study to validate HIV, HBV and syphilis screening. DBS were validated for HIV, HBV and syphilis (12), but failed when we tested for CT IgG. High background distortion gave false positive results (89-100\%) when compared to regular serum in assays from two different manufacturers (Medac, Wedel, Germany and Savyon Diagnostics, Israel). By varying the dilution factor, failure rates were reduced but still up to $25 \%$. Because of the high failure rates in DBS, we switched to an evaluation of collecting capillary blood in collection tubes as we were then able to perform the CT IgG test with regular serum (13).

Liquid capillary whole blood sent by regular mail cannot be separated from cells within the recommend two hours after blood is collected. Separation delay could result in clot-induced changes, possibly altering the analyte concentration in serum (11). Previous studies on delayed separation of whole blood showed that for a variety of analytes, samples could be stored for up to a week with only slight concentration alterations $(10,14)$.

We determined CT IgG stability in three paired capillary blood samples newly collected in serum gel tubes (BD Microtainers with clot activator and serum separator gel) exposed to room temperature for two hours, four days and seven days prior to centrifugation to simulate mail times. If CT IgG stability remained stable after four and seven days at room temperature, CT IgG concentrations could reliably be used in epidemiological studies on Chlamydia trachomatis.

\section{Methods}

Samples were collected from NECCST's participants. In NECCST, women with and without a positive CT infection history are prospectively followed for at least 10 years until 2022 (9). A preselected subset of women with known CT infection history (positive/negative) was asked to participate in this validation study. We aimed for at least 20 respondents. The study was approved by the Medical Ethical Committee Noord-Holland, Alkmaar (NL 51553.094.14/M014-042).

To test CT IgG stability, three paired samples were collected during one appointment by a medical 
professional at the VU University medical center in Amsterdam, The Netherlands, or at participant's home. Blood was collected via finger prick in BD Microtainer ${ }^{\circledR}$ blood collection tubes with clot activator and serum separator additive (SST) (Ref. 365967). We aimed for at least five droplets of whole blood per collection tube. The collected blood was stored at room temperature $\left(21^{\circ} \mathrm{C}\right)$ before centrifugation for different time intervals based on (delayed) mail delivery times. The first sample (the baseline sample, t0) was centrifuged in accordance with the guideline standard of two hours (t0), a second sample was stored for either 3,4 or 5 days ( $\mathrm{t} 1$ ), and the last sample was stored for seven days (t2). Following centrifugation, aliquoted serum was stored at $-20^{\circ} \mathrm{C}$ until analysis. Prior to analysis, frozen serum samples were thawed at room temperature and inverted several times. To avoid run-to-run variability, serum samples from all time points (t0, t1 and t2) per participant were analysed together in one batch and all samples were tested in duplicate. The CT IgG ELISA plus (CT IgG ELISA plus; Medac, Wedel, Germany) assay was used to test for CT antibodies. This is a quantitative peptide based serological assay. The ELISA was used according to the manufacturer's instructions. The mean outcome of the duplicates per sample was used in further analyses. Outcomes were reported as negative (IgG concentration <22 AU/ $\mathrm{ml}$ ), grey-zone (lgG concentration 22 - $28 \mathrm{AU} / \mathrm{ml}$ ), or positive (lgG concentration $\geq 28 \mathrm{AU} / \mathrm{ml}$ ), and quantitative in IgG concentration (AU/ml).

Laboratory results were entered in Microsoft Excel and analyzed using STATA, (version 14.2; StataCorp, College Station, TX, USA). Samples centrifuged at different time intervals (t1 and t2) were compared to the baseline sample (t0). Results were first analyzed as ordinal (i.e. negative, grey-zone and positive) using Kappa values, which indicates the level of agreement. And for calculation of the sensitivity, specificity, positive predictive value (PPV), and negative predictive value (NPV), grey-zones were classified as positive in accordance with NECCST protocol (9). Second, IgG concentrations from paired samples were analyzed using $\mathrm{r}^{2}$ 's to indicate the proportion of the variance between the baseline samples versus other time points. Additionally, Bland-Altman plots were constructed to analyze the agreement between the different time points in CT IgG concentration (15). For the Bland Altman plots upper and lower agreement limits were predefined as the margin of the grey-zone, which is -6 and $6 \mathrm{AU} / \mathrm{ml}(16,17)$. We chose $6 \mathrm{AU} / \mathrm{ml}$ because paired samples may not differ $>6 \mathrm{AU} / \mathrm{ml}$ otherwise negative samples could turn positive and vice versa. Due to the association between the difference of paired samples and the size of the measurements, raw data (including upper and lower agreement limits) were log transformed for use in Bland Altman plots (18).

\section{Results}

In total, we obtained blood from 35 women. The average age was 31.6 years (range 24-37 years) and the majority was of Western ethnicity, $77 \%$. Twenty-one women (60\%) had a positive CT infection history either by previous positive test result or self-reporting. One woman had a gonorrhoea infection in the past. We collected 98 samples, an average of 2.8 per woman. Seven women provided only two paired samples instead of three; the second sample was stored for 
either four days $(n=2)$ or seven days $(n=5)$. The amount of whole blood collected was generally small resulting in samples with a median of 40 $\mu \mathrm{l}$ serum (IQR 28.5-50.0 $\mu \mathrm{l}$ ) per collection tube. Of the 35 baseline samples, 15 (42.9\%) were CT IgG positive, 5 (14.3\%) were grey-zones and 15 (42.9\%) were negative.

Kappa values between t0 samples and, t1 and t2 samples indicated high level of agreement: 0.83 and 0.90 respectively. Three samples from the 11 and two samples from the t2 differed in outcome from to (Table 1). When grey-zones were classified as positive, only one sample was discordant. Using the latter definition sensitivity, specificity, PPV and NPV were calculated (Table 2).

Table 1: Baseline sample CT IgG outcomes compared to CT IgG outcomes from samples stored for four and seven days

\begin{tabular}{lllllllll}
\hline & \multicolumn{3}{l}{$\begin{array}{l}\text { Four days delay (t1) } \\
\mathrm{n}(\%)\end{array}$} & & $\mathrm{N}$ & $\begin{array}{l}\text { Seven days delay (t2) } \\
\mathrm{n}(\%)\end{array}$ & & $\mathrm{N}$ \\
\hline $\begin{array}{l}\text { Baseline } \\
\text { sample (t0) }\end{array}$ & Positive & Grey-zone & Negative & Total & Positive & Grey-zone & Negative & Total \\
\hline Positive & $13(100.0)$ & $0(0.0)$ & $0(0.0)$ & 13 & $14(93.3)$ & $1(6.7)$ & $0(0.0)$ & 15 \\
Grey-zone & $2(50.0)$ & $2(50.0)$ & $0(0.0)$ & 4 & $1(25.0)$ & $3(75.0)$ & $0(0.0)$ & 4 \\
Negative & $0(0.0)$ & $1(7.7)$ & $12(92.3)$ & 13 & $0(0.0)$ & $0(0.0)$ & $14(100.0)$ & 14 \\
\hline Total & $15(50.0)$ & $3(10.0)$ & $12(40.0)$ & 30 & $14(43.8)$ & $4(12.5)$ & $14(43.8)$ & 33 \\
\hline
\end{tabular}

Table 2: Sensitivity, specificity, positive predictive value and negative predictive value

\begin{tabular}{|c|c|c|c|c|c|c|c|c|c|}
\hline & $N$ & $\begin{array}{c}\text { True } \\
\text { positive } \\
\text { (n) }\end{array}$ & $\begin{array}{c}\text { False } \\
\text { positive }\end{array}$ & $\begin{array}{c}\text { True } \\
\text { negative }\end{array}$ & $\begin{array}{c}\text { False } \\
\text { negative }\end{array}$ & $\begin{array}{c}\text { Sensitivity } \\
(\%)\end{array}$ & $\begin{array}{l}\text { Specificity } \\
(\%)\end{array}$ & $\begin{array}{l}\text { PPV } \\
(\%)\end{array}$ & $\begin{array}{l}\text { NPV } \\
(\%)\end{array}$ \\
\hline t1 & 30 & 17 & 1 & 12 & 0 & 100 & 92 & 94 & 100 \\
\hline t2 & 33 & 19 & 0 & 14 & 0 & 100 & 100 & 100 & 100 \\
\hline
\end{tabular}

${ }^{*}$ For these analyses grey zone values were considered as positive values. PPV $=$ positive predictive value. $\mathrm{NPV}=$ negative predictive value.

Figure 1 shows the concentration of the baseline samples compared to the concentration of the $t 1$ and t2 samples, r2's were both 0.99. Bland Altman plots show all values to be within the predefined limits of agreement. 

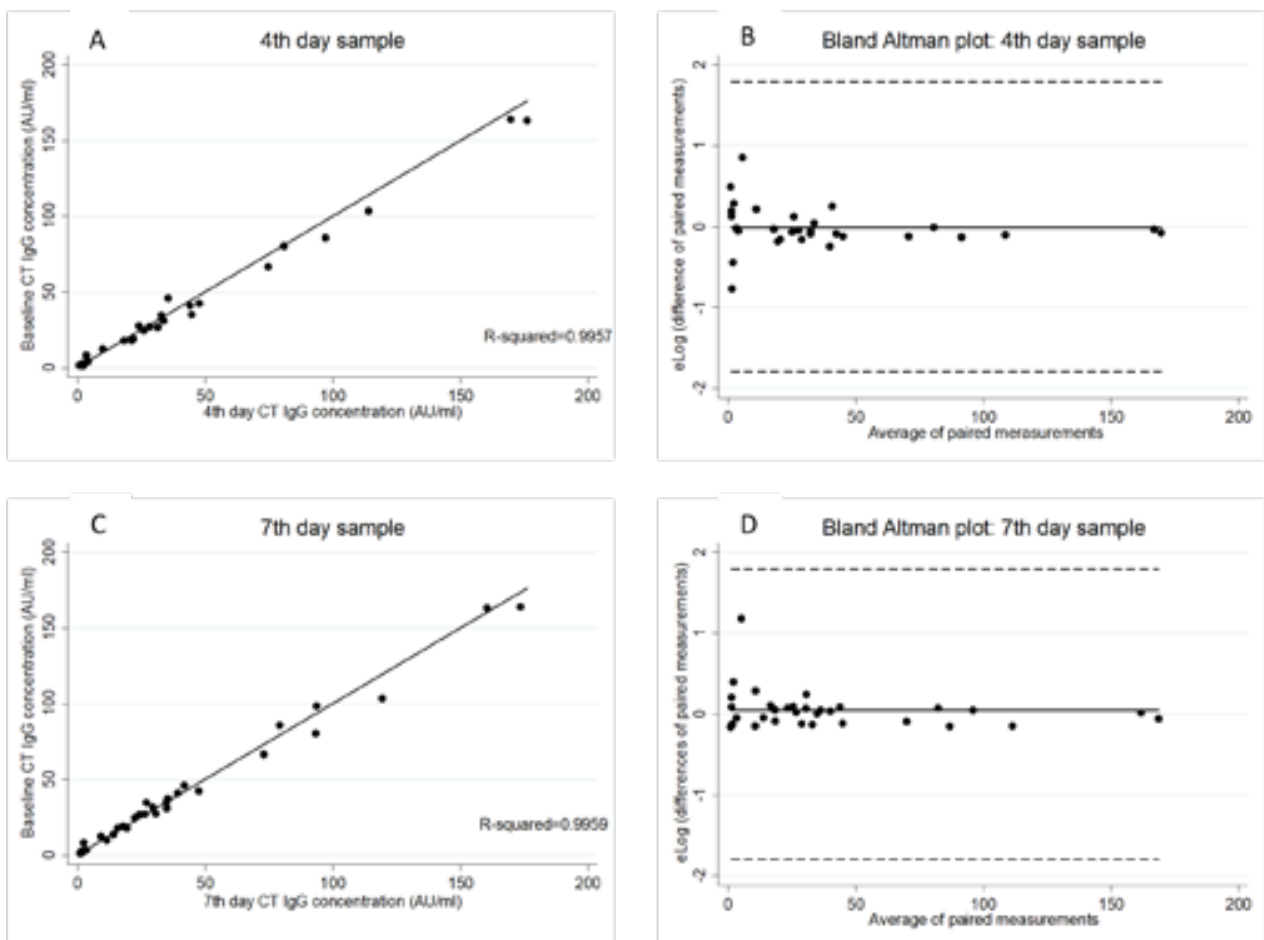

Figure 1. Graphs A. and C. Show scatterplots with regression lines of baseline sample concentrations plotted against 4th day $(A)$ and 7th day $(C)$ delayed samples including r-squared coefficients. Graphs B and $\mathrm{D}$ show Bland-Altman plots, based on log-transformed data, in which elog difference between the baseline sample and 4th day (B) or 7th day (D) sample concentration were plotted against the average of paired measurements. Upper and lower limits (dashed lines) in the Bland-Altman plots were based on clinically relevant elog(-6 and $6 \mathrm{AU} / \mathrm{ml})$ values.

\section{Discussion}

This is the first study to investigate stability of CT IgG concentration in samples with separation delay stored in BD Microtainers. The SST tubes cause blood to clot rapidly, enabling the separation of the blood clot from serum (19). The high sensitivity, specificity, PPV, and NPV and $\mathrm{r}^{2}$ 's of almost one showed that CT IgG concentrations in serum remain stable up to seven days after whole blood collection. Therefore, this method can be used in epidemiological studies.

In this study, samples were stored in the laboratory to simulate delayed mail times, but kept in temperature controlled rooms. In the actual NECCST cohort, samples are sent using regular mail; this means samples will be exposed to more variable temperatures, including day/night and day-to-day differences, which may result in lower stability. However, other studies determining the effect of centrifugation delay on a variety of analytes that compared various storage temperatures also showed that total protein levels, including IgG levels, often only change by a few percentages after 24 hours to seven days of delay $(10,20)$. 
Home-collected biological materials such as urine samples and vaginal swab samples are already implemented in CT studies and screening (21-24). Home-sampling increases participation rates and provides a less costly alternative for clinic-testing. Even though only small amounts of blood can be collected, in general this proved to be enough to perform reliable serology tests for detection of CT IgG antibodies. Following results of this study, we conclude blood collected at home in collection tubes and sent to laboratories can reliably be used for Chlamydia trachomatis IgG measurements. 


\section{References}

1. Low N, McCarthy A, Macleod J, Salisbury C, Campbell R, Roberts TE, et al. Epidemiological, social, diagnostic and economic evaluation of population screening for genital chlamydial infection. Health Technol Assess. 2007;11(8):iiiiv, ix-xii, 1-165.

2. Davies B, Turner KM, Frolund M, Ward H, May MT, Rasmussen S, et al. Risk of reproductive complications following chlamydia testing: a population-based retrospective cohort study in Denmark. Lancet Infect Dis. 2016.

3. Centers for Disease C, Prevention. Recommendations for the laboratory-based detection of Chlamydia trachomatis and Neisseria gonorrhoeae--2014. MMWR Recomm Rep. 2014;63(RR-02):1-19.

4. Gijsen AP, Land JA, Goossens VJ, Slobbe ME, 12. Bruggeman CA. Chlamydia antibody testing in screening for tubal factor subfertility: the significance of $\lg G$ antibody decline over time. Hum Reprod. 2002;17(3):699-703.

5. Clad A, Freidank HM, Kunze M, Schnoeckel $U$, Hofmeier S, Flecken $U$, et al. Detection of seroconversion and persistence of Chlamydia trachomatis antibodies in five different serological tests. Eur J Clin Microbiol Infect Dis. 2000;19(12):932-7.

6. Morre SA, Munk C, Persson K, Kruger-Kjaer $S$, van Dijk R, Meijer CJ, et al. Comparison of three commercially available peptide-based immunoglobulin $G$ ( $\lg G$ ) and $\lg A$ assays to microimmunofluorescence assay for detection of Chlamydia trachomatis antibodies. J Clin Microbiol. 2002;40(2):584-7.

7. Horner PJ, Wills GS, Reynolds R, Johnson AM, Muir DA, Winston A, et al. Effect of time since exposure to Chlamydia trachomatis on chlamydia antibody detection in women: a cross-sectional study. Sex Transm Infect. 2013;89(5):398-403.

8. Johnson AM, Horner P. A new role for Chlamydia trachomatis serology? Sex Transm Infect. 2008;84(2):79-80.
Hoenderboom BM, van Oeffelen AA, van Benthem BH, van Bergen JE, Dukers-Muijrers $\mathrm{NH}$, Gotz HM, et al. The Netherlands Chlamydia cohort study (NECCST) protocol to assess the risk of late complications following Chlamydia trachomatis infection in women. BMC Infect Dis. 2017;17(1):264

10. Clark S, Youngman LD, Palmer A, Parish S, Peto R, Collins R. Stability of plasma analytes after delayed separation of whole blood: implications for epidemiological studies. International Journal of Epidemiology. 2003;32(1):125-30.

11. Holland NT, Smith MT, Eskenazi B, Bastaki M. Biological sample collection and processing for molecular epidemiological studies. Mutat Res. 2003;543(3):217-34.

van Loo IHM, Dukers-Muijrers N, Heuts R, van der Sande MAB, Hoebe C. Screening for HIV, hepatitis $B$ and syphilis on dried blood spots: A promising method to better reach hidden highrisk populations with self-collected sampling. PLoS One. 2017;12(10):e0186722.

13. Liu Y, Rafkin LE, Matheson D, Henderson C, Boulware D, Besser RE, et al. Use of selfcollected capillary blood samples for islet autoantibody screening in relatives: a feasibility and acceptability study. Diabet Med. 2017.

14. Ikeda K, Ichihara K, Hashiguchi T, Hidaka Y, Kang D, Maekawa $M$, et al. Evaluation of the short-term stability of specimens for clinical laboratory testing. Biopreserv Biobank. 2015;13(2):135-43.

15. Altman D.G. BJM. Measurement in Medicine: The Analysis of Method Comparison Studies. Journal of the Royal Statistical Society. 1983;32(3):307-17.

16. Bland JM, Altman DG. Measuring agreement in method comparison studies. Stat Methods Med Res. 1999;8(2):135-60.

17. Giavarina D. Understanding Bland Altman analysis. Biochem Med (Zagreb). 2015;25(2):141-51.

18. 18. Scott LE, Galpin JS, Glencross DK. 
Multiple method comparison: statistical model using percentage similarity. Cytometry B Clin Cytom. 2003;54(1):46-53.

19. Bowen RA, Remaley AT. Interferences from blood collection tube components on clinical chemistry assays. Biochem Med (Zagreb). 2014;24(1):31-44.

20. Tanner M, Kent N, Smith B, Fletcher S, Lewer M. Stability of common biochemical analytes in serum gel tubes subjected to various storage temperatures and times pre-centrifugation. Ann Clin Biochem. 2008;45(Pt 4):375-9.

21. Morre $S A$, van Valkengoed $I G$, de Jong $A$, Boeke AJ, van Eijk JT, Meijer CJ, et al. Mailed, home-obtained urine specimens: a reliable screening approach for detecting asymptomatic Chlamydia trachomatis infections. J Clin Microbiol. 1999;37(4):976-80.

22. Doshi JS, Power J, Allen E. Acceptability of chlamydia screening using self-taken vaginal swabs. Int J STD AIDS. 2008;19(8):507-9.

23. van den Broek IV, van Bergen JE, Brouwers EE, Fennema JS, Gotz HM, Hoebe CJ, et al. Effectiveness of yearly, register based screening for chlamydia in the Netherlands: controlled trial with randomised stepped wedge implementation. BMJ. 2012;345:e4316.

24. Hocking JS, Guy R, Walker J, Tabrizi SN. Advances in sampling and screening for chlamydia. Future Microbiol. 2013;8(3):367-86. 


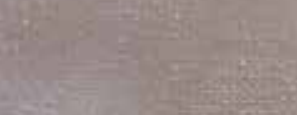

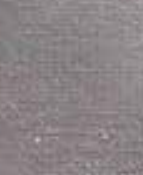

\section{sisting}

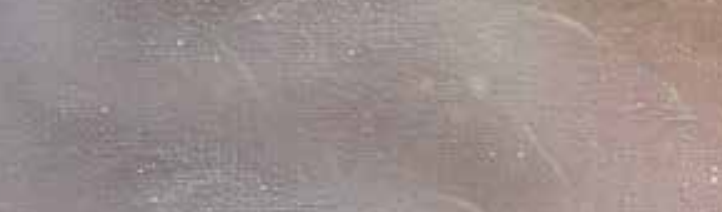

जince

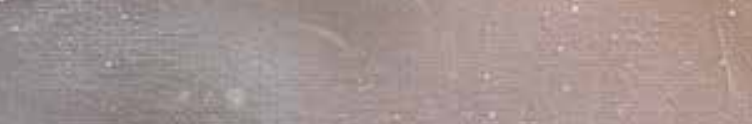

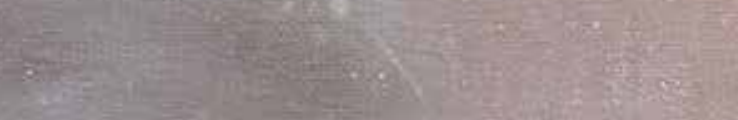

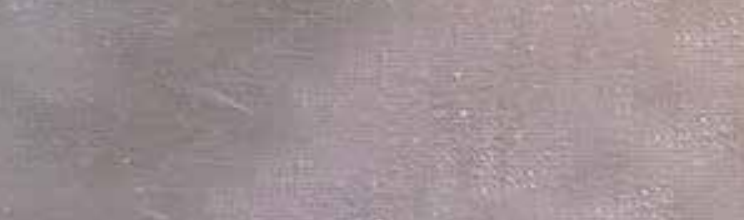

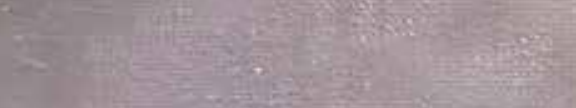

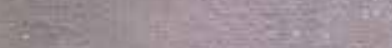

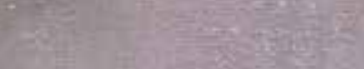

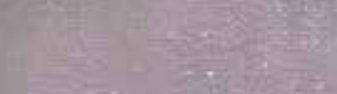

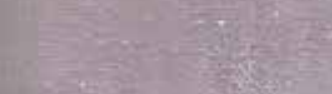

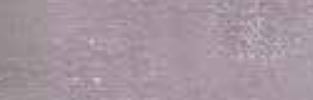

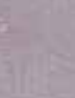

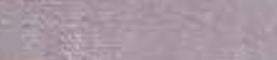

netwo

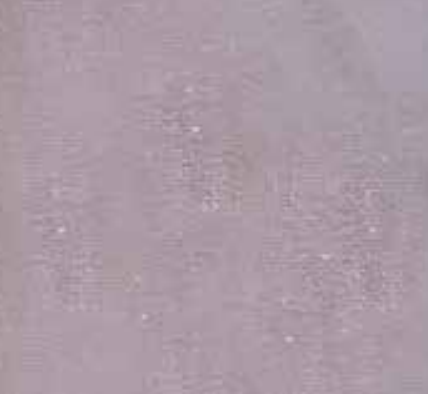

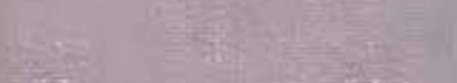

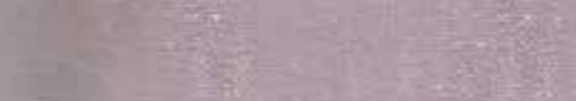




\section{4}

Can previous associations of single nucleotide polymorphisms in the TLR2, NOD1, CXCR5, and IL10 genes in the susceptibility to and severity of Chlamydia trachomatis infections be confirmed?

Jelmer B Jukema, Bernice M Hoenderboom, Birgit HB van Benthem, Marianne A van der Sande, Henry JC de vries, Christian JPA Hoebe, Nicole HTM Dukers-Muijrers, Caroline J Bax, Servaas A Morré, Sander Ouburg

Published in: Pathogens 2021; 10.3390/pathogens10010048 


\section{Abstract}

Clear inter-individual differences exist in the response to $C$. trachomatis (CT) infections and reproductive tract complications in women. Host genetic variation like single nucleotide polymorphisms (SNPs) has been associated with differences in response to CT infection, and SNPS might be used as a genetic component in a tubal-pathology predicting algorithm. Our aim was to confirm the role of four genes by investigating proven associated SNPs in the susceptibility and severity of a CT infection. A total of 1201 women from five cohorts were genotyped and analyzed for TLR2 + 2477 G>A, NOD1 + 32656 T->GG, CXCR5 + 10950 T >C, and IL10 -1082 A > G. Results confirmed that NOD1 $+32656 \mathrm{~T}->\mathrm{GG}$ was associated with an increased risk of an symptomatic CT infection (OR: 1.9, 95\% $\mathrm{Cl}: 1.1-3.4, p=0.02$ ), but we did not observe an association with late complications. IL10-1082 A>G appeared to increase the risk of late complications (i.e., ectopic pregnancy/tubal factor infertility) following a CT infection ( $\mathrm{OR}=2.8,95 \% \mathrm{Cl}: 1.1-7.1, p=0.02)$. Other associations were not found. Confirmatory studies are important and large cohorts are warranted to further investigate the role of SNPs in the susceptibility and severity of a CT infection. 


\section{Introduction}

Chlamydia trachomatis (CT) is the most diagnosed bacterial sexually transmittable infection (STI) worldwide [1], with an estimated 131 million new infections each year [2]. In the Netherlands, approximately 60.000 new infections occur on a yearly basis [3]. In women, an estimated $70-80 \%$ of the infections are asymptomatic [4]. These women are thus at risk to remain untreated; leaving them prone to late complications such as pelvic inflammatory disease (PID), ectopic pregnancy $(E P)$, and tubal factor infertility (TFI) $[1,5]$. Estimating the individual risk of late complications is complicated by interpersonal differences in susceptibility, course, and outcome of the infection. These differences in women can at least to some extent be explained by bacterial factors (e.g., virulence, load), environmental factors (e.g., co-infection, microbiome), and host factors (e.g., immunogenetic differences between individuals, (sexual) risk behaviour) $[6,7]$.

Since CT is assumed to be an important cause of tubal pathology [8], subfertile women in the Netherlands who attend a fertility specialist are tested with a chlamydia antibody test (CAT) which can identify a past infection. If the CAT is positive a hysterosalpingogram (HSG) is performed to examine the tubes and if the HSG is indicative for tubal pathology a laparoscopy, which is the golden standard, follows. However, since the CAT is designed to detect a past infection, and not to identify tubal pathology, it has a suboptimal predictive value for finding tubal pathology. This may lead to incorrect triage and thus to unnecessary tubal imaging. These invasive tests are uncomfortable, come with health hazards, and are expensive. Therefore there is a need for more specific markers to identify increased risk for tubal pathology.

In addition to serology markers, host genetics could be important in the risk for tubal pathology. A considerable part of the interpersonal differences in responding to a CT infection can be explained by host genetics. A twin study has suggested that almost $40 \%$ of the difference in the immunological response to CT infection is based on host genetics [9]. A large number of Single Nucleotide Polymorphisms (SNPS) has been linked to differences in the susceptibility to and severity of an CT infection [10]. The most relevant SNPs for CT are in intra- and extra cellular pathogen recognition receptors (PRRs), and in cytokines and chemokines involved in and modulating the immune response after infection with CT [11]. Some SNPs result in an enhanced risk for infection or complications after CT infection, while others lower the risk for infection or complications. Hence, a proposed way of improving current fertility work-up is the development of a tubal-pathology predicting algorithm based on host genetics in combination with serology [12].

Four well described SNPs that have previously been associated with the outcomes of a CT infection are: TLR2 + 2477 G >A (rs5743708), NOD1 + 32656 T-> GG (rs6958571), CXCR5 + 10950 $\mathrm{T}>\mathrm{C}$ (rs3922), and IL10 -1082 A>G (rs1800896). TLR2 has been shown to play an important role as a mediator in the innate immune response to a CT infection. It has also been shown to be important in the early production of inflammatory mediators and the development of chronic inflammatory pathology [13]. Verweij et al., found that $T L R 2+2477{ }^{\star} A$ provided an increased risk for the development of tubal pathology in CT positive women (OR 17.5) [14], although the study group was rather small. CT seropositive women carrying the NOD1 GG insertion had a 
more than twice increased risk of tubal pathology (OR: 2.25; 95\% Cl: 1.08-4.67, $p=0.04$ ) [15]. NOD1 normally functions as an intracellular pattern receptor but the GG insertion creates a stop codon thus impairing the functioning of the gene. In contrast to the TLR2 and NOD1 mutations, the SNP in the CXCR5 gene was protective. CXCR5 CC had a large protective effect for CT positive women (OR: 0.1, 95\% Cl: 0.04-0.5, $p=0.002$ ) against developing tubal pathology[16]. And finally, the A allele of the $/ L 10-1082 \mathrm{G}>\mathrm{A}$ SNP, which resides in the promotor region of this immunosuppressive cytokine, protected against the development of severe tubal damage [17]. The aim of this study is to build the evidence base for the role of human genes in CT infection, and assess to what extent the earlier described associations of SNPs in four genes in the susceptibility to and severity of a CT infection using clinically well-defined cohorts could be confirmed.

\section{Materials and Methods}

\section{Studied Cohorts}

A total of 1201 women from four different STI and one late CT complication cohorts were included in this study which aimed to confirm the role of SNPs in the four genes, IL10, NOD1, TLR2, and CXCR5, in the susceptibility to and severity (which was separated in symptomatic course and late complications) of CT infection in women. Characteristics of the five cohorts are listed in Table 1. Cohorts 1-3 were used to test susceptibility and cohorts $1,2,4$ and 5 to test severity of CT infection. Cohort 3 only contained information about CT status, not severity of infection, hence, it was not included in the severity analysis. Cohorts 4 and 5 consisted only of CT positive women and were therefore not included in the susceptibility analyses. From all cohorts only women from West-European ethnicity (i.e., Dutch, British, Austrian, Belgian, German, Irish, or Luxembourgish) were included. From all samples of included women, DNA was isolated for SNP determination, as listed in table 1. Sample material was either serum, a buccal swab, a vaginal swab, urine, or PBMC (peripheral blood mononuclear cell). 


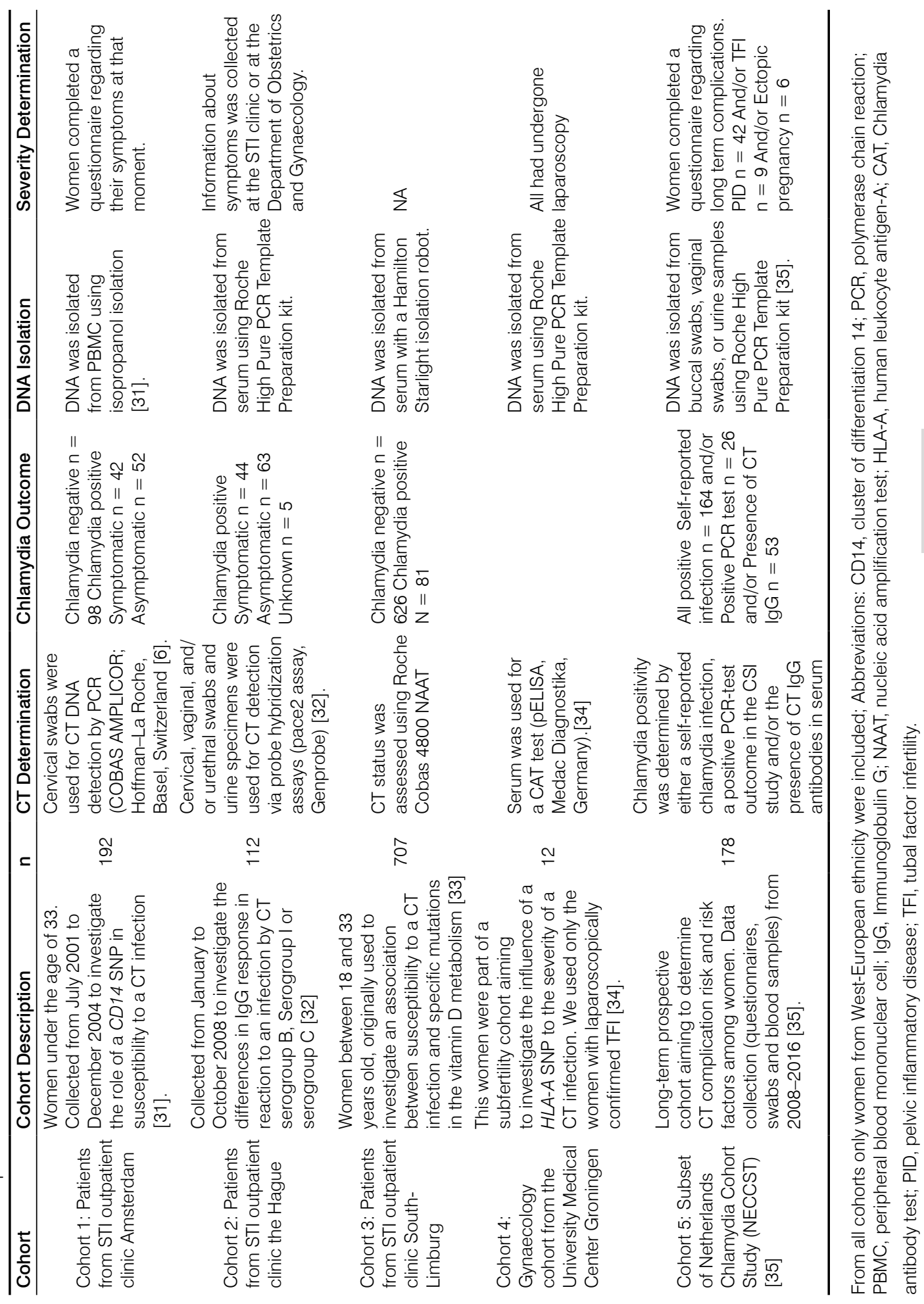


Since SNP analysis requires large datasets, also a percentage of women who had already previously been tested for these SNPs was included to maximise the amount of data. For three out of four SNPs (NOD1 + 32656 T->GG, TLR2 +2477 G>A, and CXCR5 +10950 T>C) the susceptibility to a CT infection had previously been studied using (part of) cohort 1. In these previous studies, NOD1 and TLR2 used a different outcome, i.e., including CT serology positivity. [14-16] For the current confirmation study two cohorts have been added, making the percentages of overlap between the current and the previous studies $11 \%, 19 \%, 19 \%$, and $0 \%$ for NOD 1 , TLR2, CXCR5 and IL10 respectively. For severity, two SNPS (NOD1 + 32656 T->GG and TLR2 $+2477 \mathrm{G}>\mathrm{A}$ ) in part used the same cohorts as the original research; cohort 1 was previously used for symptomatology assessment in NOD1 (26\% overlap) (14) and TLR2 (47\% overlap) (13). Furthermore $21 \%$ of cases (and $0 \%$ of controls) for late complications match with the original paper for NOD1 (14). The majority (86\%) of SNP determinations was done on women not previously tested for these SNPS.

\section{SNP Determination}

The isolated DNA samples were used to determine the SNPs TLR2 +2477 G>A (rs5743708), NOD1 +32656 T-> GG (rs6958571), CXCR5 + 10950 T >C (rs3922), and IL10 -1082 A>G (rs1800896). The SNPs were genotyped either at LGC in the United Kingdom (cohort 1-4), or in our own laboratory of Immunogenetics VUmc, The Netherlands (cohort 5) using KASP (Kompetitive Allele Specific PCR) technology. [36] This technology was supplemented with in-house RT-PCR using Roche Assay-by-Design.

\section{Data Analyses}

Susceptibility Analyses: Cohorts 1-3

Susceptibility to CT infection and the presence of the four SNPs was determined in cohorts 1-3. Cases were defined as tested PCR-positive for CT DNA during STI clinic visit. Controls were participants who had tested PCR-negative for CT during STI clinic visit.

Genotype distributions were tested in all cohorts and between cohorts. In case genotype distribution did not differ significantly between cohorts ( $\chi^{2}$ test), cohorts were combined to increase power. Analyses were performed for all four SNPs separately. Subsequently a multivariable logistic regression was performed on all four SNPs to evaluate whether SNPs would be predictive for the susceptibility of CT infections independent of other SNPs. Occurrence of SNPs in cases and controls was compared using $\chi^{2}$ tests and risks of CT acquisition between different SNP distributions were described as odds ratio's (OR) with 95\% confidence interval $(\mathrm{Cl})$.

\section{Severity Analyses: Cohorts 1-5}

The definition of severity of CT infection can be divided in two ways: 1) as an immediate symptomatic course of infection \& 2) as late complications (i.e., pelvic inflammatory disease, ectopic pregnancy and/or tubal factor infertility). Cohort 1 and 2 contained information regarding symptomatic course of infection and cohort 4 and 5 contained information regarding late complication. The severity of CT infection and the presence of SNPs was assessed in four ways. 
I: Investigating severity in terms of symptomatic CT infections versus asymptomatic CT infections, determining the presence of the SNPs in cohorts 1 and 2. Cases were defined as symptomatic CT positive women and controls as asymptomatic CT positive women.

II: Examining the presence of SNPs among CT positive women, comparing women positive for CT complications to women negative for CT complications. Cases were defined as women with a positive CT history and PID and/or ectopic pregnancy and/or TFI. In sensitivity analyses, PID was excluded in the definition to create a more specific outcome (due to heterogeneity in PID diagnosis). Cases were defined as women with a positive CT history and ectopic pregnancy and/ or TFI. In cohort 4, TFI was defined as extensive peri-adnexal adhesions and/or distal occlusion of at least one tube, not attributable to abdominal pathology other than the genital tract infection (e.g., appendicitis). [34] In Cohort 5 self-reported TFI was used. Controls were defined as women with a positive CT history without PID, ectopic pregnancy, and TFI and with at least one pregnancy of $>20$ weeks.

III: Performing trend analysis (i.e., a statistical procedure performed to evaluate hypothesized linear and nonlinear relationships between quantitative variables) to study the relationship between SNP occurrence and increased severity among cohorts 4 and 5 . The hypothesis that the percentage of people carrying the risk genotypes would increase with increasing severity was tested. The groups, which were compared for trend were, arranged in order of severity: fertile CT positive women (i.e., pregnant for at least once for $>20$ weeks), CT positive women with PID, CT positive women with ectopic pregnancy and/or tubal factor infertility.

IV: It is well possible that SNPs in the different pathways do overlap and that women have multiple SNPS. Multiple SNPs can interact and therefore the result of having multiple SNPs might be different compared to just assessing all SNPs apart. To correct for this we applied multivariable logistic regression to all four SNPs to evaluate whether the combination of SNPs would be predictive for the severity of CT infections. Analyses were performed on cohort 1 and 2 combined to assess the predictive value for symptomatology as well as on cohort 4 and 5 to assess the predictive value for long-term complications.

\section{Sensitivity Analysis}

If the analysis involved women who previously have been tested for the described SNPs, a sensitivity analysis was performed excluding these women (Appendix Table A1-A4, Appendix figure A1). No sensitivity analysis regarding the susceptibility could be performed for TLR2 and CXCR5. Cohort 1 had, in full, already been used to evaluate these SNPs. Sensitivity analysis using only cohort 2 proved impossible since this cohort existed only of CT positive women. Therefore a different kind of sensitivity analysis was done comparing the CT positive women of cohort 2 with the CT negative women of cohort 1. In this way a new comparison was made between cases who were never tested for the SNP and controls who were (Appendix Table A1).

$\chi^{2}$ tests were used and risks were described as odds ratio's (OR) with 95\% confidence interval $(\mathrm{Cl})$. $P$ values $<0.05$ were considered statistically significant. Analyses were performed using IBM SPSS Statistics. Regression coefficient $\left(R^{2}\right)$ for the trends was calculated using an ordinal scale in Microsoft Office Excel. 


\section{METC Approval}

The act 'Medical Research Involving Human Subjects' (WMO, Dutch Law) states that anonymous spare human materials and data may be used for research purposes if the data are completely anonymised and not retrievable. Cohort 5 was approved by Medical Ethical Committee VU medical Center, Amsterdam the Netherlands (NL 51553.094.14/2015.903(A2019.336)). All participants provided informed consent for participation.

\section{Results}

DNA Isolation and SNP Determination

SNP determination for cohort 1-4 was done by LGC, UK. Genotyping success rate for the four SNPs in these cohorts ranged from $77.6 \%$ to $99.1 \%$. For cohort 5, 178 samples were isolated and genotyped in-house. SNP determination of all four SNPs was successful for 162 DNA samples (91\%). All SNPs were present in the cohorts, with TLR2 having the lowest minor allele frequency of $6.2 \%$

\section{Susceptibility to CT Infection}

The genotype distribution based on CT status is shown in Table 2. Genotype distribution in cohort 3 differed significantly from genotype distribution in cohort 1 and 2 . Hence cohort 1 and $2(\mathrm{~N}=304)$ were combined for this analysis and cohort $3(\mathrm{~N}=707)$ was analyzed separately. A difference in the genotype distributions of $I L 10-1082 A>G$ was found in cohort $3(p=0.05)$. For women carrying $I L 10{ }^{*} \mathrm{G}$ risk of CT infection was slightly lower compared to women who were homozygous wildtype, but this did not reach statistical significance (OR 0.6, 95\%Cl: 0.3-1.2, $p=$ 0.14 ). No statistically significant associations were observed (all $p>0.3$ ) between the three other studied SNPs and the susceptibility to a CT infection, Table 4. This also remained unchanged in the sensitivity analysis in which a fraction of cohort 1 for NOD1 was excluded. The area under the curve (AUC) of the regression models for the susceptibility analysis in cohort 1 and 2 was 0.52 (95\% Cl: 0.45-0.59), and 0.57 (95\% Cl: 0.50-0.63) for cohort 3. 
Table 2. Genotype distribution by CT status

\begin{tabular}{|c|c|c|c|c|c|c|c|c|c|c|c|c|}
\hline \multirow[t]{2}{*}{ Susceptibility } & \multicolumn{3}{|c|}{ IL-10-1082 } & \multicolumn{3}{|c|}{ NOD1 + 32656} & \multicolumn{3}{|c|}{$T L R 2+2477$} & \multicolumn{3}{|c|}{ CXCR5 + 10950} \\
\hline & AA & $A G$ & GG & TT & TGG & GGGG & GG & GA & AA & TT & TC & $\mathrm{CC}$ \\
\hline Cohorts 1,2 & $\begin{array}{l}92 \\
(30.3 \%)\end{array}$ & $\begin{array}{l}133 \\
(43.8 \%)\end{array}$ & $\begin{array}{l}79 \\
(26.0 \%)\end{array}$ & $\begin{array}{l}167 \\
(54.9 \%)\end{array}$ & $\begin{array}{l}121 \\
(39.8 \%) \\
\end{array}$ & (5.3\%) & $\begin{array}{l}280 \\
(92.1 \%) \\
\end{array}$ & $\begin{array}{l}24 \\
(7.9 \%) \\
\end{array}$ & $0(0 \%)$ & $\begin{array}{l}111 \\
(36.5 \%)\end{array}$ & $\begin{array}{l}146 \\
(48.0 \%)\end{array}$ & $\begin{array}{l}47 \\
(15.5 \%)\end{array}$ \\
\hline CT Negative & $\begin{array}{l}34 \\
(34.7 \%)\end{array}$ & $\begin{array}{l}38 \\
(38.8 \%)\end{array}$ & $\begin{array}{l}26 \\
(26.5 \%)\end{array}$ & $\begin{array}{l}52 \\
(53.1 \%)\end{array}$ & $\begin{array}{l}42 \\
(42.9 \%))\end{array}$ & $\begin{array}{l}4 \\
(4.1 \%)\end{array}$ & $\begin{array}{l}89 \\
(90.8 \%)\end{array}$ & $\begin{array}{l}9 \\
(9.2 \%)\end{array}$ & $0(0 \%)$ & $\begin{array}{l}35 \\
(35.7 \%)\end{array}$ & $\begin{array}{l}50 \\
(51.0 \%)\end{array}$ & $\begin{array}{l}13 \\
(13.3 \%)\end{array}$ \\
\hline & $\begin{array}{l}58 \\
(28.2 \%) \\
\end{array}$ & $\begin{array}{l}95 \\
(46.1 \%) \\
\end{array}$ & $\begin{array}{l}53 \\
(25.7 \%) \\
\end{array}$ & $\begin{array}{l}115 \\
(55,8 \%) \\
\end{array}$ & $\begin{array}{l}79 \\
(38.3 \%) \\
\end{array}$ & $\begin{array}{l}12 \\
(5.8 \%) \\
\end{array}$ & $\begin{array}{l}191 \\
(92.7 \%) \\
\end{array}$ & $\begin{array}{l}15 \\
(7.3 \%)) \\
\end{array}$ & $\%)$ & $\begin{array}{l}76 \\
(36.9 \%) \\
\end{array}$ & $\begin{array}{l}96 \\
(46.6 \%) \\
\end{array}$ & $\begin{array}{l}34 \\
(16.5 \%) \\
\end{array}$ \\
\hline & AA & AG & GG & TT & TGG & GGGG & GG & GA & AA & TT & TC & $\mathrm{CC}$ \\
\hline Cohort 3 & $\begin{array}{l}202 \\
(28.6 \%) \\
\end{array}$ & $\begin{array}{l}365 \\
(51.6 \%)\end{array}$ & $\begin{array}{l}140 \\
(19.8 \%)\end{array}$ & $\begin{array}{l}427 \\
(60.4 \%)\end{array}$ & $\begin{array}{l}229 \\
(32.4 \%)\end{array}$ & $\begin{array}{l}51 \\
(7.2 \%) \\
\end{array}$ & $\begin{array}{l}674 \\
(95.3 \%) \\
\end{array}$ & $\begin{array}{l}30 \\
(4.2 \%) \\
\end{array}$ & $\begin{array}{l}3 \\
(0.4 \%) \\
\end{array}$ & $\begin{array}{l}246 \\
(34.8 \%) \\
\end{array}$ & $\begin{array}{l}338 \\
(47.8 \%)\end{array}$ & $\begin{array}{l}123 \\
(17.4 \%) \\
\end{array}$ \\
\hline CT Negative & $\begin{array}{l}184 \\
(29.4 \%)\end{array}$ & $\begin{array}{l}313 \\
(50.0 \%)\end{array}$ & $\begin{array}{l}129 \\
(20.6 \%)\end{array}$ & $\begin{array}{l}374 \\
(59.7 \%)\end{array}$ & $\begin{array}{l}204 \\
(32.6 \%)\end{array}$ & $\begin{array}{l}48 \\
(7.7 \%)\end{array}$ & $\begin{array}{l}596 \\
(95.2 \%)\end{array}$ & $\begin{array}{l}27 \\
(4.3 \%)\end{array}$ & $\begin{array}{l}3 \\
(0.5 \%)\end{array}$ & $\begin{array}{l}216 \\
(34.5 \%)\end{array}$ & $\begin{array}{l}300 \\
(47.9 \%)\end{array}$ & $\begin{array}{l}110 \\
(17.6 \%)\end{array}$ \\
\hline Pos & $\begin{array}{l}18 \\
(22.2 \%)\end{array}$ & $\begin{array}{l}52 \\
(64.2 \%)\end{array}$ & $\begin{array}{l}11 \\
(13.6 \%)\end{array}$ & $\begin{array}{l}53 \\
(65.4 \%)\end{array}$ & $\begin{array}{l}25 \\
\text { (30.9\%) }\end{array}$ & $\begin{array}{l}3 \\
(3.7 \%)\end{array}$ & $\begin{array}{l}78 \\
(96.3 \%)\end{array}$ & $\begin{array}{l}3 \\
(3.7 \%)\end{array}$ & $0(0 \%)$ & $\begin{array}{l}30 \\
(37.0 \%)\end{array}$ & $\begin{array}{l}38 \\
(46.9 \%)\end{array}$ & $\begin{array}{l}13 \\
(16.0 \%)\end{array}$ \\
\hline
\end{tabular}

Abbreviation: CT, Chlamydia trachomatis. No significant differences were found between any of the distributions for chlamydia positive and negative women.

\section{Severity of CT Infection}

In Table 3, the SNP genotype data are given used for the severity analyses to CT infection.

I: We observed that CT positive women with the NOD1 GG insertion were more likely to have a symptomatic course of infection (OR: 1.9, 95\%Cl: 1.1-3.4, $p=0.02$ ) as compared to an asymptomatic infection. This association remained unchanged in sensitivity analysis (Appendix Table A2\&A4). Carriage of TLR2 $+2477^{\star} A$ approached significance when assessing CT positive women with a symptomatic course of infection compared to CT positive women without one (OR: $2.6,95 \% \mathrm{Cl}: 0.8-8.0, p=0.10$ ). The other two SNPs were not statistically associated with the severity of infection (Table 5).

II: In women with and without late complications from cohort 4 and 5 we did not observe significant differences in SNP distributions, although IL10 approached statistical significance. CT positive women carrying IL10 GG had a marginally, but not statistically significant, increased risk for developing complications after a CT infection (OR: 1.9, 95\%Cl: 1.0-3.6, $p=0.07$ ) (Table 5). When comparing CT positive women with ectopic pregnancy/TFI to the fertile CT controls (excluding PID cases) the women carrying IL10 GG had a significant higher risk of developing late complications $(\mathrm{OR}=2.8,95 \% \mathrm{Cl}: 1.1-7.1, p=0.02)$. No associations for the other SNPs were found in this analysis.

III: Trend analysis using cohorts 4 and 5 are shown in Figure 1. IL10 GG showed an $R^{2}$ of 0.92 $(p=0.07)$. Carriage of the GG genotype was more common among women with increased severity: $28 \%$ for fertile CT positive women to $34 \%$ for CT positive with PID to $52 \%$ for CT positive women with ectopic pregnancy/tubal factor infertility. No such association was observed for the other SNPs in this analysis. 
Table 3. Genotype distribution by severity of infection.

\begin{tabular}{|c|c|c|c|c|c|c|c|c|c|c|c|c|}
\hline \multirow{2}{*}{$\begin{array}{l}\text { Severity } \\
\text { Analysis I: } \\
\text { Cohorts } 1,2\end{array}$} & \multicolumn{3}{|c|}{ IL-10-1082 } & \multicolumn{3}{|c|}{ NOD1 + 32656} & \multicolumn{3}{|c|}{$T L R 2+2477$} & \multicolumn{3}{|c|}{ CXCR5 + 10950} \\
\hline & $A A$ & $A G$ & GG & TT & TGG & GGGG & GG & GA & AA & TT & TC & $\mathrm{CC}$ \\
\hline CT + total & $\begin{array}{l}56 \\
(27.9 \%)\end{array}$ & $\begin{array}{l}94 \\
(46.8 \%)\end{array}$ & $\begin{array}{l}51 \\
(25.4 \%)\end{array}$ & $\begin{array}{l}112 \\
(55.7 \%)\end{array}$ & $\begin{array}{l}77 \\
(38,3 \%)\end{array}$ & $\begin{array}{l}12 \\
(6.0 \%)\end{array}$ & $\begin{array}{l}187 \\
(93.0 \%)\end{array}$ & $\begin{array}{l}14 \\
(7.0 \%))\end{array}$ & $\begin{array}{l}0 \\
(0 \%)\end{array}$ & $\begin{array}{l}74 \\
(36.8 \%)\end{array}$ & $\begin{array}{l}94 \\
(46.8 \%)\end{array}$ & $\begin{array}{l}33 \\
(16.4 \%)\end{array}$ \\
\hline $\mathrm{CT}+\mathrm{AS}$ & $\begin{array}{l}34 \\
(29.6 \%)\end{array}$ & $\begin{array}{l}51 \\
(44.3 \%)\end{array}$ & $\begin{array}{l}30 \\
(26.1 \%)\end{array}$ & $\begin{array}{l}72 \\
(62.6 \%)\end{array}$ & $\begin{array}{l}36 \\
(31.3 \%)\end{array}$ & $\begin{array}{l}7 \\
(6.1 \%)\end{array}$ & $\begin{array}{l}110 \\
(95.7 \%)\end{array}$ & $\begin{array}{l}5 \\
(4.3 \%)\end{array}$ & $\begin{array}{l}0 \\
(0 \%)\end{array}$ & $\begin{array}{l}42 \\
(36.5 \%)\end{array}$ & $\begin{array}{l}55 \\
(47.8 \%)\end{array}$ & $\begin{array}{l}18 \\
(15.7 \%)\end{array}$ \\
\hline $\mathrm{CT}+\mathrm{S}$ & $\begin{array}{l}22 \\
(25.6 \%)\end{array}$ & $\begin{array}{l}43 \\
(45.7 \%)\end{array}$ & $\begin{array}{l}21 \\
(24.4 \%)\end{array}$ & $\begin{array}{l}40 \\
(46.5 \%)\end{array}$ & $\begin{array}{l}41 \\
(47.7 \%)\end{array}$ & $\begin{array}{l}5 \\
(5.8 \%)\end{array}$ & $\begin{array}{l}77 \\
(89.5 \%)\end{array}$ & $\begin{array}{l}9 \\
(10.5 \%)\end{array}$ & $\begin{array}{l}0 \\
(0 \%)\end{array}$ & $\begin{array}{l}32 \\
(37.2 \%)\end{array}$ & $\begin{array}{l}39 \\
(45.4 \%)\end{array}$ & $\begin{array}{l}15 \\
(17.4 \%)\end{array}$ \\
\hline$P$ value & \multicolumn{3}{|c|}{$P$ for $G G$ vs $A^{*}=0.79$} & \multicolumn{3}{|c|}{$P$ for ${ }^{*} \mathrm{GG}$ vs TT $=0.02^{* *}$} & \multicolumn{3}{|c|}{$P$ for ${ }^{\star} A$ vs $G G=0.09$} & \multicolumn{3}{|c|}{$P$ for $C C$ vs $T^{\star}=0.74$} \\
\hline $\begin{array}{l}\text { Analysis II: } \\
\text { Cohorts } 4,5\end{array}$ & $A A$ & AG & GG & TT & TGG & GGGG & GG & GA & AA & TT & TC & $\mathrm{CC}$ \\
\hline Total & $\begin{array}{l}45 \\
(25.9 \%)\end{array}$ & $\begin{array}{l}73 \\
(42.0 \%)\end{array}$ & $\begin{array}{l}56 \\
(32.2 \%)\end{array}$ & $\begin{array}{l}102 \\
(58.6 \%)\end{array}$ & $\begin{array}{l}64 \\
(36.8 \%)\end{array}$ & $\begin{array}{l}8 \\
(4.6 \%)\end{array}$ & $\begin{array}{l}165 \\
(94.8 \%)\end{array}$ & $\begin{array}{l}7 \\
(4.0 \%)\end{array}$ & $\begin{array}{l}2 \\
(1.1 \%)\end{array}$ & $\begin{array}{l}63 \\
(36.2 \%)\end{array}$ & $\begin{array}{l}80 \\
(46.0 \%)\end{array}$ & $\begin{array}{l}31 \\
(17.8 \%)\end{array}$ \\
\hline Controls & $\begin{array}{l}31 \\
(26.7 \%)\end{array}$ & $\begin{array}{l}53 \\
(45.7 \%)\end{array}$ & $\begin{array}{l}32 \\
(27.6 \%)\end{array}$ & $\begin{array}{l}69 \\
(59.5 \%)\end{array}$ & $\begin{array}{l}40 \\
(34.5 \%)\end{array}$ & $\begin{array}{l}7 \\
(6.0 \%)\end{array}$ & $\begin{array}{l}109 \\
(94.0 \%)\end{array}$ & $\begin{array}{l}5 \\
(4.3 \%)\end{array}$ & $\begin{array}{l}2 \\
(1.7 \%)\end{array}$ & $\begin{array}{l}44 \\
(37.9 \%)\end{array}$ & $\begin{array}{l}55 \\
(47.4 \%)\end{array}$ & $\begin{array}{l}17 \\
(14.7 \%)\end{array}$ \\
\hline Cases & $\begin{array}{l}14 \\
(24.1 \%)\end{array}$ & $\begin{array}{l}20 \\
(34.5 \%)\end{array}$ & $\begin{array}{l}24 \\
(41.4 \%)\end{array}$ & $\begin{array}{l}33 \\
(56.9 \%)\end{array}$ & $\begin{array}{l}24 \\
(41.4 \%)\end{array}$ & $\begin{array}{l}1 \\
(1.7 \%)\end{array}$ & $\begin{array}{l}56 \\
(96,6 \%)\end{array}$ & $\begin{array}{l}2 \\
(3,4 \%)\end{array}$ & $\begin{array}{l}0 \\
(0.0 \%)\end{array}$ & $\begin{array}{l}19 \\
(32.8 \%)\end{array}$ & $\begin{array}{l}25 \\
(43.1 \%)\end{array}$ & $\begin{array}{l}14 \\
(24.1 \%)\end{array}$ \\
\hline$P$ value & \multicolumn{3}{|c|}{$P$ for $G G$ vs $A^{*}=0.07$} & \multicolumn{3}{|c|}{$P$ for ${ }^{*} G G$ vs TT $=0.74$} & \multicolumn{3}{|c|}{$P$ for ${ }^{\star} A$ vs $G G=0.47$} & \multicolumn{3}{|c|}{$P$ for $C C$ vs $T^{*}=0.12$} \\
\hline
\end{tabular}

$P$ values based on chi-square, significant results are marked with ** Abbreviations: CT+, Chlamydia trachomatis positive; AS, asymptomatic; S, symptomatic

IV: The AUC for the regression model performed on all four SNPs comparing symptomatic vs asymptomatic in cohort 1 and 2 was 0.60 (95\% Cl: $0.52-0.68)$. The same score of 0.60 was obtained in the analysis of cohort 4 and 5 (95\% Cl: 0.52-0.69). After correcting results for the other investigated SNPs similar results as to the uncorrected data were found (Table 4 and 5).

Table 4. Crude and adjusted results for susceptibility to infection

\begin{tabular}{|c|c|c|c|c|c|}
\hline \multicolumn{2}{|c|}{ Susceptibility } & \multirow{2}{*}{$\frac{\text { IL10: GG vs. A * }}{1.0(95 \% \mathrm{Cl}: 0.6-1.7)}$} & \multirow{2}{*}{$\frac{\text { NOD1: * GG vs. TT }}{0.9(95 \% \mathrm{Cl}: 0.6-1.4)}$} & \multirow{2}{*}{$\begin{array}{l}\text { TLR2: * A vs. GG } \\
0.8 \text { (95\%Cl: } 0.3-1.8)\end{array}$} & \multirow{2}{*}{$\begin{array}{l}\text { CXCR5: * C vs TT } \\
1.0(95 \% \text { Cl: } 0.6-1.6)\end{array}$} \\
\hline Cohorts 1,2 & OR crude & & & & \\
\hline & $P$ value crude & 0.88 & 0.65 & 0.57 & 0.84 \\
\hline & OR MLR & 1.0 (95\%Cl: 0.6-1.7) & 0.9 (95\%Cl: 0.6-1.5) & 0.8 (95\%Cl: 0.3-1.9 & 1.0 (95\%Cl: 0.6-1.6) \\
\hline & $P$ value MLR & 0.90 & 0.69 & 0.60 & 0.85 \\
\hline \multirow[t]{4}{*}{ Cohort 3} & OR crude & 0.6 (95\%Cl: 0.3-1.2) & 0.8 (95\%Cl: 0.5-1.3) & 0.8 (95\%Cl: 0.3-2.9) & 0.9 (95\%Cl: 0.6-1.4) \\
\hline & $P$ value crude & 0.14 & 0.33 & 0.79 & 0.65 \\
\hline & OR MLR & 0.6 (95\%Cl: 0.3-1.2) & 0.8 (95\%Cl: $0.5-1.3)$ & 0.9 (95\%Cl: 0.3-2.9) & 0.9 (95\%Cl: $0.6-1.5)$ \\
\hline & $P$ value MLR & 0.15 & 0.32 & 0.82 & 0.66 \\
\hline
\end{tabular}

Abbreviations: MLR, multivariable logistic regression 
Table 5. Crude and adjusted results for severity of infection

\begin{tabular}{|c|c|c|c|c|c|}
\hline Severity & & IL10: GG vs. A * & NOD1: * GG vs. TT & TLR2: * A vs. GG & CXCR5: CC vs. ${ }^{*} \mathrm{~T}$ \\
\hline \multirow[t]{4}{*}{ Cohorts 1,2} & OR crude & 0.9 (95\%Cl: $0.5-1.7)$ & $1.9(95 \% \mathrm{Cl}: 1.1-3.4)$ & 2.6 (95\%Cl: 0.8-8.0) & $1.1(95 \% \mathrm{Cl}: 0.5-2.4)$ \\
\hline & $P$ value crude & 0.79 & $0.02^{* *}$ & 0.10 & 0.73 \\
\hline & OR MLR & 0.9 (95\%Cl: $0.5-1.7)$ & 1.9 (95\%Cl: $1.1-3.4)$ & 2.4 (95\%Cl: $0.8-7.5)$ & 1.1 (95\%Cl: 0.5-2.4) \\
\hline & $P$ value MLR & 0.68 & $0.03^{\star \star}$ & 0.14 & 0.79 \\
\hline \multirow[t]{4}{*}{ Cohorts 4,5} & OR crude & 1.9 (95\%Cl: $1.0-3.6)$ & $1.1(95 \% \mathrm{Cl}: 0.6-2.1))$ & 0.6 (95\% Cl: $0.1-2.8)$ & 1.9 (95\%Cl: $0.8-4.1$ \\
\hline & $P$ value crude & 0.07 & 0.74 & 0.47 & 0.12 \\
\hline & OR MLR & 1.9 (95\%Cl: $0.9-3.6)$ & 1.3 (95\%Cl: $0.6-2.4)$ & 0.5 (95\% Cl: $0.1-2.7)$ & 1.9 (95\%Cl: $0.8-4.2)$ \\
\hline & $P$ value MLR & 0.07 & 0.50 & 0.43 & 0.13 \\
\hline
\end{tabular}

Significant results are marked with ** Abbreviations: MLR, multivariable logistic regression
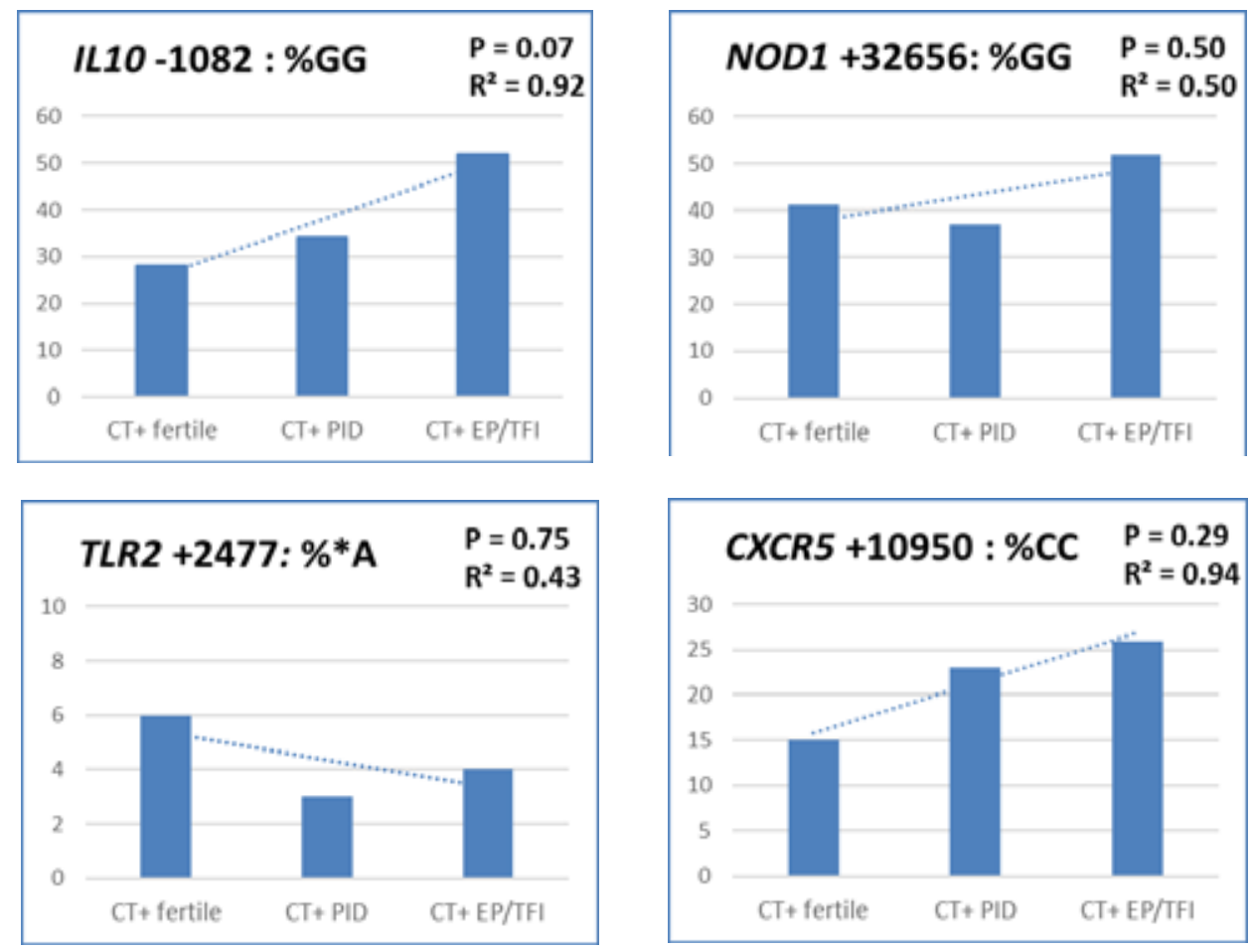

Figure 1. Trend analysis of Cohorts (4) and (5) Comparing genotype distributions between women based on increased severity: (1) Fertile CT positive women. (2) CT positive women with PID. (3) CT positive women with ectopic pregnancy and/or tubal factor infertility. Percentage of genotypes as part of total. Abbreviations: $\mathrm{CT}+$, Chlamydia trachomatis positive; PID, pelvic inflammatory disease; EP, ectopic pregnancy; TFI, tubal factor infertility. 


\section{Discussion}

The aim of this study was to add to the current evidence base on the role of human genes in CT infection, by assessing whether we could confirm the previously observed role of SNPs in the genes IL10, NOD1, TLR2, and CXCR5 in the susceptibility to and severity of a CT infection. These four genes are all involved in detecting micro-organisms and starting the inflammatory response. Disease pathology is based on the functionality of these four genes linked to the SNPs studies. Meaning the severity of infection is genetically based in individuals. The novelty of this study is confined to confirming already proven associations with new and more data. Confirmation of these previous associations would be one step further in the direction of using these SNPs as a genetic part of a tubal pathology predicting algorithm. This algorithm will aim to differentiate between women likely to have fertility problems due to CT infections and women without increased risk. Saving women without increased risk a unnecessary tubal imaging will save them an uncomfortable, expensive invasive test with health hazards. Also, an earlier tubal imaging of women with a genetically very high risk of infertility due to CT can save a couple trying to become pregnant in vain. On the other hand, women without CT antibodies (and thus a low risk to tubal pathology) but with a high genetic risk profile should be investigated in more detail instead on trying to become pregnant for a year longer._This is due to two potential effects: (1) loss of antibodies to CT and (2) tubal pathology due to other STDs like Neisseria for which the SNP algorithm potentially also works.

The viability of such an tubal pathology predicting algorithm is still subject to scientific debate. Earlier attempts in other complex diseases to use polygenic risk scores which were based on small numbers of highly significant SNPs identified from GWA studies achieved only limited predictive value [18]. However, this algorithm will not only be based on genetics. Current machine learning methods allow for unprecedented pattern detection in both genetics and other factors. Other factors could possibly include interaction between genetic variants an different disease serovars, amount of infections, co-infections, treatment (failure), age, birthplace, how positive the CAD test turns out \& sexual behavior. For now, this manuscript focusses solely on the genetic component and more specifically the confirmation of four previously proven SNP's. If such a mentioned algorithm will never be created, or if it does not employ host genetics, at the very least this study aids in further uncovering host factors driving ascension and pathology.

An association with susceptibility for CT infections was previously found for NOD1 + 32656 T>GG but could not be confirmed in the current study. In contrast, the association of IL10 -1082 A>G with susceptibility was not earlier investigated, where we observed a protective effect. However, when comparing carriers of the mutation vs. homozygote wild-type, significance was lost (aOR $0.6, p=0.15)$.

In the severity analysis we confirmed the role for NOD1 + 32656 T>GG; NOD1 + 32656 * GG associated with a twofold higher risk of a symptomatic course of CT infection. CT Positive women with IL10 -1082 GG had an almost threefold higher odds ratio for developing late complications (i.e., EP/TFI), compared to CT positive females with the $A^{*}$ genotype. When including PID in the analysis, as well as in trend analysis comparing fertile CT positive women to CT positive women 
with PID to CT positive women with EP/TFI, a near significant $(p=0.07)$ association was found for IL10-1082 GG. The role of the SNPs TLR2 + 2477 G>A and CXCR5 +10950 T>C in the severity of infection could not be confirmed in this study.

NOD1 normally functions as an intracellular pattern receptor and is capable of triggering the host innate immune signaling pathways. This results in the production of pro-inflammatory cytokines, which are a vital part of the host defense against CT [19]. However, NOD1 + 32656 T>GG creates a stop codon thus impairing the functioning of the gene and the host defense. Brankoviç et al., found a protective effect for NOD1 +32656 * GG (OR 0.52; 95\%Cl: 0.32-0.83, $p=0.006$ ) in the susceptibility to infection[15] while we did not find an association between this SNP and the susceptibility to a CT infection. A plausible explanation for this difference is that Brankoviç used a more strict definition. Our definition is positivity for CT DNA, while his research only used women who were both CT DNA and CT IgG positive, compared to women negative for both. When assessing the severity of a CT infection, Brankoviç's study found that carrying the NOD1 GG insertion increased risk of tubal pathology (OR: 2.25; 95\% Cl: 1.08-4.67, $p=0.04$ ). When comparing CT-positive women without symptoms to CT-positive women with symptoms to CT-positive women with TFI, Brankoviç found an increasing trend in carriage of the GG allele (p-trend: 0.0003). While we could confirm the NOD1 GG insertion association with a symptomatic course of a CT infection, we did not find an association between the GG insertion and late complications. Concerning the OR for late complications, an important difference between Brankoviç's research and ours is that Brankoviç reported on CT positive women diagnosed with TFI and we, in this analysis, did not take the CT status in account. The difference in results in trend analysis are probably also due to definition differences. Brankoviç compared asymptomatic women to symptomatic women to women with TFI (all CT positive) while we compared fertile women to women with PID to women with EP/TFI (all CT positive).

Our result of $I L 10-1082 A>G$ being a risk SNP is contrary to our hypothesis which was based on research done by Ohman et al., They showed that the A allele was significantly associated with increased disease severity after CT infection [17]. Also other research indicated the AA genotype as risk factor for Chlamydial TFI [20]. The GG genotype was found in $41.1 \%$ of our cases, while Ohman had found $19.8 \%$. The AA genotype was found in $24.1 \%$ and $29.2 \%$ for our study and Ohman's study respectively, a remarkable difference in genotype distributions for cases in different populations. A possible explanation could be that genotype distribution of the IL-10 -1082 SNP in Finland is quite different compared to our West-European population [17]. The results we found for $I L 10$ are seemingly contradicting when looking at the protective effect for $I L 10-1082{ }^{*} G$ in the susceptibility to CT infection versus the risk and role of the IL10-1082 GG genotype in the severity of a CT infection. An explanation could be that these are clearly two different stages in complications of CT infections. It has been shown that $I L 10$ suppresses the inflammatory functions of macrophages, NK cells, dendritic cells, Th1, Th2, and B lymphocytes by regulating the expression of interferon- $\gamma$, tumour necrosis factor- $\alpha$, major histocompatibility complex class II antigens, and co-stimulatory molecules, making it one of the most important regulatory factors. [21-23] The IL10-1082 SNP, which resides in the promotor region, forms three haplotypes with two other SNPs in this promotor region: -819 C > T and -592 C > A. The haplotypes 
formed are: GCC, ACC, and ATA. ACC and ATA are generally linked with low cytokine production; GCC is linked with a high IL10 production [24,25]. However, results differ per study and also the reverse has been suggested [26,27]. For our study it could be hypothesized that if GG is the genotype with a low IL10 production then the lack of suppression (especially of interferon- $\gamma$ [25]) will upregulate the host defense against intracellular infections, clearing the infection at an early stage. But, if the upregulated immune system is unable to clear the infection it might be stimulated too much, resulting in enhanced inflammation and tissue damage and thus increasing the chance of episodes of PID and potentially subsequent tubal scarring. The absence of an association between susceptibility and TLR2 $+2477 \mathrm{G}>\mathrm{A}$ could be explained by earlier studies, which found it only associated in haplotype combinations [14]. The haplotypes with an increased risk of infection were heterozygous (GA) or homozygous (GG) for +2477 SNP [14]. In our study TLR2 approached significance when comparing CT positive women with a symptomatic course of infection to CT positive women with an asymptomatic course of infection. In the original research, using cohort 1, no such a near-significant value was found.[14] In the study by Verweij et al. TLR2 +2477 *A was also more frequently present in patients with tubal pathology (19.2\%) compared to women without tubal pathology $(0 \%, p=0.015)$ [14]. The mechanism explaining these associations might be a lowered responsiveness to lipoproteins by the mutation [28], making it more difficult to recognise the CT particle.

CXCR5 has mainly been studied in mice so far, in which it appears to regulate CD4- and natural killer T-cells [16]. The CXCR5 +10950 CC genotype of this chemokine receptor has been found to protect CT positive women with an OR of 0.1 of developing tubal pathology.[16] In our study, this finding could not be confirmed. Consistent with the previous findings [16], it did not associate with an altered susceptibility to a CT infection.

Several limitations can be noted for this study. First, even though the women with late complications were selected out of large cohorts the total number in the end is still relatively small. Second, for power purposes we decided to include as many people as possible and thus confirmed earlier results using partly the same participants. However, sensitivity analysis yielded similar results. This sensitivity analysis were done using only the women who had never been tested before for these SNPs. Or, if this method was not possible, comparing never before used cases with already analysed controls. Third, the SNP distribution in cohort 3 did not match the SNP distribution of cohort 1 and 2 ( $p$ for difference between groups $\leq 0.05$ ). This means we needed to assess the susceptibility in cohort 1 and 2 combined and 3 separately, resulting in an unexpected loss of power. The difference between the SNP distributions could be the different geographical location of cohort 3 inside the Netherlands. A study employing whole-genome sequencing to investigate variation and population structure in the Netherlands identified non-random sharing of rare mutations within and across provinces [29]. Also it used principal component analysis of common SNPs (frequency $>5 \%$ ) to show a subtle substructure along a north-south gradient in the Netherlands [29]. Fourth, the definition of PID is a difficult one and as can be seen in Figure 1 the PID group does not always follow the same expected trend when trend analysing with increasing severity are performed, even though CT is linked to PID. Fifth, we could not correct for co-infections as we do not have this data. Therefore we can't be absolutely certain that late complications are not caused by co-infections like Gonorrhoea. However, this prevalence in Holland is low [30]. 
Considering cohort 1-3 a limitation is that susceptibility was only measured at one point in time. We could not confirm all prior findings. This shows on one hand that confirmatory studies are of high importance and on the other hand that larger studies to further investigate these four, and other SNPs, are warranted. The ultimate goal of these studies is to determine the potential of these SNPs as a genetic component of a tubal-pathology prediction algorithm among CT positive women. The aim of the algorithm is twofold. First to minimize the number of infertile women who try to become pregnant naturally, while actually IVF is indicated. Second, to reduce the number of fertile women unnecessarily undergoing a laparoscopy. In conclusion, our research does not exclude that genetics may in part be associated with the susceptibility and severity of CT infections, however, there is insufficient evidence to justify routine determination of the genetic signature of the four studies SNP's in clinical practice yet. More research, for these SNP's and other genetic variations, to provide more insight seems needed. 


\section{References}

1. Lal, J.A., et al., Chlamydia trachomatis infections and subfertility: opportunities to translate host pathogen genomic data into public health. Public Health Genomics, 2013. 16(1-2): p. 50-61.

2. WHO. Sexually transmitted infections (STIS). 2016; Available from: http://www.who.int/ mediacentre/factsheets/fs110/en/.

3. Slurink, I.A., et al, Sexually transmitted infections in the Netherlands in 2018. 2019, National Institute for Public Health and the Environment.

4. Black, C.M., Current methods of laboratory diagnosis of Chlamydia trachomatis infections. Clin Microbiol Rev, 1997. 10(1): p. 160-84.

5. den Hartog, J.E., et al., Do host genetic traits in the bacterial sensing system play a role in the development of Chlamydia trachomatisassociated tubal pathology in subfertile women? BMC Infect Dis, 2006. 6: p. 122.

6. Spaargaren, J., A multidisciplinary approach to the study of Chlamydia trachomatis infections. Female urogenital and male anorectal infections, in VUMC labaratory of immunogenetics. 2006, VU Amsterdam: www.immunogenetics.nl.

7. den Hartog, J.E., et al., TLR4 in Chlamydia trachomatis infections: knockout mice, STD patients and women with tubal factor subfertility. Drugs Today (Barc), 2009. 45 Suppl B: p. 75-82.

8. Hoenderboom, B.M., et al., Relation between Chlamydia trachomatis infection and pelvic inflammatory disease, ectopic pregnancy and tubal factor infertility in a Dutch cohort of women previously tested for chlamydia in a chlamydia screening trial. Sex Transm Infect, 2019. 95(4): p. 300-306.

9. Bailey, R.L., et al., Host genetic contribution to the cellular immune response to Chlamydia trachomatis: Heritability estimate from a Gambian twin study. Drugs Today (Barc), 2009. 45 Suppl B: p. 45-50.

10. Morre, S.A., O. Karimi, and S. Ouburg, Chlamydia trachomatis: identification of susceptibility markers for ocular and sexually transmitted infection by immunogenetics. FEMS Immunol Med Microbiol, 2009. 55(2): p. 140-53.

11. Mascellino, M.T., P. Boccia, and A. Oliva, Immunopathogenesis in Chlamydia trachomatis Infected Women. ISRN Obstet Gynecol, 2011. 2011: p. 436936.

12. Broeze, K.A., et al., Integration of patient characteristics and the results of Chlamydia antibody testing and hysterosalpingography in the diagnosis of tubal pathology: an individual patient data meta-analysis. Hum Reprod, 2012. 27(10): p. 2979-90.

13. Darville, T., et al., Toll-like receptor-2, but not Toll-like receptor-4, is essential for development of oviduct pathology in chlamydial genital tract infection. J Immunol, 2003. 171(11): p. 6187-97.

14. Verweij, S.P., et al., TLR2, TLR4 and TLR9 genotypes and haplotypes in the susceptibility to and clinical course of Chlamydia trachomatis infections in Dutch women. Pathog Dis, 2016. 74(1): p. ftv107.

15. Brankovic, I., et al., NOD1 in contrast to NOD2 functional polymorphism influence Chlamydia trachomatis infection and the risk of tubal factor infertility. Pathog Dis, 2015. 73(1): p. 1-9.

16. Karimi, O.J.J.O., S; Morré, SA, CXCR5 regulates Chlamydia tubal pahtology in mice and humans. 2011.

17. Ohman, H., et al., Cytokine polymorphisms and severity of tubal damage in women with Chlamydia-associated infertility. J Infect Dis, 2009. 199(9): p. 1353-9.

18. Ho, D.S.W., et al., Machine Learning SNP Based Prediction for Precision Medicine. Front Genet, 2019. 10: p. 267.

19. Zou, Y., et al., The role of NOD1 and NOD2 in host defense against chlamydial infection. FEMS Microbiol Lett, 2016. 363(17)

20. Kinnunen, A.H., et al., HLA DQ alleles and interleukin-10 polymorphism associated with Chlamydia trachomatis-related tubal factor infertility: a case-control study. Hum Reprod, 
2002. 17(8): p. 2073-8.

21. Sabat, R., et al., Biology of interleukin-10. Cytokine Growth Factor Rev, 2010. 21(5): p. 331-44.

22. Scapini, P., et al., B cell-derived IL-10 suppresses inflammatory disease in Lyn-deficient mice. Proc Natl Acad Sci U S A, 2011. 108(41): p. E823-32.

23. Moore, K.W et at Interleukin-10 and the interleukin-10 receptor. Annu Rev Immunol, 2001. 19: p. 683-765.

24. Turner, D.M., et al., An investigation of polymorphism in the interleukin-10 gene promoter. Eur J Immunogenet, 1997. 24(1): p. 1-8.

25. Ohman, H., et al., IL-10 polymorphism and cell-mediated immune response to Chlamydia trachomatis. Genes Immun, 2006. 7(3): p. 2439.

26. Gibson, A.W., et al., Novel single nucleotide polymorphisms in the distal IL-10 promoter affect IL-10 production and enhance the risk of systemic lupus erythematosus. J Immunol, 2001. 166(6): p. 3915-22.

27. Wang, C., et al., Human leukocyte antigen and cytokine gene variants as predictors of recurrent Chlamydia trachomatis infection 35 in high-risk adolescents. J Infect Dis, 2005. 191(7): p. 1084-92.

28. Lorenz, E., et al., A novel polymorphism in the toll-like receptor 2 gene and its potential association with staphylococcal infection. Infect Immun, 2000. 68(11): p. 6398-401.

29. Genome of the Netherlands, C., Whole-genome sequence variation, population structure and demographic history of the Dutch population. Nat Genet, 2014. 46(8): p. 818-25

30. Staritsky, L., et al., Sexually transmitted infections in the Netherlands in 2019, in Seksueel overdraagbare aandoeningen in Nederland in 2019. 2020, Rijksinstituut voor Volksgezondheid en Milieu RIVM.

31. Ouburg, S., et al., The CD14 functional gene polymorphism -260 C > T is not involved in either the susceptibility to Chlamydia trachomatis infection or the development of tubal pathology. BMC Infect Dis, 2005. 5: p. 114.

32. Verweij, S.P., et al., Significantly higher serologic responses of Chlamydia trachomatis B group serovars versus $C$ and I serogroups. Drugs Today (Barc), 2009. 45 Suppl B: p. 135-40.

33. Lanjouw, E., et al., Specific polymorphisms in the vitamin $\mathrm{D}$ metabolism pathway are not associated with susceptibility to Chlamydia trachomatis infection in humans. Pathog Dis, 2016. 74(3)

34. Jansen, M.E., et al., Potential protective effect of a $G>A$ SNP in the 3'UTR of HLA-A for Chlamydia trachomatis symptomatology and severity of infection. Pathog Dis, 2016. 74(2).

35. Hoenderboom, B.M., et al., The Netherlands Chlamydia cohort study (NECCST) protocol to assess the risk of late complications following Chlamydia trachomatis infection in women. BMC Infect Dis, 2017. 17(1): p. 264.

36. He, C., J. Holme, and J. Anthony, SNP genotyping: the KASP assay. Methods Mol Biol, 2014. 1145: p. 75-86. 


\section{Supplementary material}

Table A1 Sensitivity analyses

\begin{tabular}{|c|c|c|c|c|c|c|c|c|c|c|c|}
\hline \multirow{2}{*}{\multicolumn{2}{|c|}{$\begin{array}{l}\text { Susceptibility } \\
\text { Sensivisity } \\
\text { analysis }\end{array}$}} & \multicolumn{4}{|c|}{ NOD1 + 32656} & \multicolumn{3}{|c|}{$T L R 2+2477$} & \multicolumn{3}{|c|}{ CXCR5 + 10950} \\
\hline & & TT & TGG & $G G$ & & $G G$ & $\Delta$ & $A$ & TT & $\mathrm{TC}$ & $\mathrm{CC}$ \\
\hline & $\begin{array}{l}1 \\
\text { on), } 2\end{array}$ & $\begin{array}{l}103 \\
(53.6 \%) \\
\end{array}$ & $\begin{array}{l}79 \\
(41.1 \%) \\
\end{array}$ & $\begin{array}{l}10 \\
(5.2 \%) \\
\end{array}$ & s), 2 & $\begin{array}{l}191 \\
(91.1 \%) \\
\end{array}$ & 6) & $\begin{array}{l}0 \\
(0.0 \%) \\
\end{array}$ & $\begin{array}{l}77 \\
(36.7 \%) \\
\end{array}$ & $\begin{array}{l}103 \\
(49.0 \%) \\
\end{array}$ & $(14.3 \%)$ \\
\hline \multirow[t]{2}{*}{ CT } & iveg & $\begin{array}{l}20 \\
(51.3 \%)\end{array}$ & $\begin{array}{l}18 \\
(46.2 \%)\end{array}$ & $\begin{array}{l}1 \\
(2.6 \%)\end{array}$ & Negative & $\begin{array}{l}89 \\
(90.8 \%)\end{array}$ & 9 & $\begin{array}{l}0 \\
(0.0 \%)\end{array}$ & $\begin{array}{l}35 \\
(35.5 \%)\end{array}$ & $\begin{array}{l}50 \\
(51.0 \%)\end{array}$ & $\begin{array}{l}13 \\
(13.3 \%)\end{array}$ \\
\hline & Positive & $\begin{array}{l}83 \\
(54.2 \%)\end{array}$ & $\begin{array}{l}61 \\
(39.9 \%)\end{array}$ & $\begin{array}{l}9 \\
(5.6 \%)\end{array}$ & 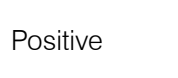 & $\begin{array}{l}102 \\
(91.1 \%)\end{array}$ & $\begin{array}{l}10 \\
(8.9 \%)\end{array}$ & $\begin{array}{l}0 \\
(0.0 \%)\end{array}$ & $\begin{array}{l}42 \\
(37.5 \%)\end{array}$ & $\begin{array}{l}53 \\
(47.3 \%)\end{array}$ & $\begin{array}{l}17 \\
(15.2 \%)\end{array}$ \\
\hline
\end{tabular}

Abbreviations: CT, Chlamydia trachomatis

Table A2 Sensitivity analysis

\begin{tabular}{|c|c|c|c|c|c|c|c|}
\hline \multirow{2}{*}{$\begin{array}{l}\text { Severity } \\
\text { Analysis I: } \\
\text { Cohorts } 1 \text { (selection), } 2\end{array}$} & \multicolumn{4}{|c|}{$N O D 1+32656$} & \multicolumn{3}{|c|}{$T L R 2+2477$} \\
\hline & TT & TGG & GGGG & $\begin{array}{l}\text { Analysis I: } \\
\text { Cohort } 2\end{array}$ & GG & GA & AA \\
\hline $\mathrm{CT}+$ total & $80(54.1 \%)$ & 59 (39.9\%) & $9(6.1 \%)$ & \multirow{3}{*}{$\begin{array}{l}C T+\text { total } \\
C T+A S \\
C T+S\end{array}$} & $98(91.6 \%)$ & $9(8.4 \%))$ & $0(0 \%)$ \\
\hline $\mathrm{CT}+\mathrm{AS}$ & $55(61.1 \%)$ & 29 (32.2\%) & $6(6.7 \%)$ & & $60(95.2 \%)$ & $3(4.8 \%)$ & $0(0 \%)$ \\
\hline $\mathrm{CT}+\mathrm{S}$ & $25(43.1 \%)$ & $30(51.7 \%)$ & $3(5.2 \%))$ & & $38(86.4 \%)$ & $6(13.6 \%)$ & $0(0 \%)$ \\
\hline$P$ value & \multicolumn{3}{|c|}{$P$ for * GG vs TT $=0.03$ ** } & $P$ value & \multicolumn{3}{|c|}{$P$ for *A vs GG $=0.08$} \\
\hline Analysis II: Cohort 5 & TT & TGG & GGGG & & & & \\
\hline Total & 97 (59.9\%) & 57 (35.2\%) & $8(4.9 \%)$ & & & & \\
\hline Controls & 69 (59.5\%) & $40(34.5 \%)$ & $7(6.0 \%)$ & & & & \\
\hline Cases & $28(60.9 \%)$ & $17(37.0 \%)$ & $1(2.2 \%)$ & & & & \\
\hline$P$ value & \multicolumn{3}{|c|}{ for ${ }^{*} G G$ vs TT $=0.87$} & & & & \\
\hline
\end{tabular}

Sensitivity analysis, $P$ values based on chi-square, significant results are marked with ** Abbreviations: $\mathrm{CT}+$, Chlamydia trachomatis positive; AS, asymptomatic; $\mathrm{S}$, symptomatic

Table A3 Sensitivity analysis

\begin{tabular}{lll}
\hline Susceptibility & & NOD1: ${ }^{*}$ GG vs TT \\
\hline Cohorts & OR crude & $0.9(95 \% \mathrm{Cl}: 0.4-1.8)$ \\
1 (selection), 2 & P value crude & 0.74 \\
& OR MLR & $0.9(95 \% \mathrm{Cl}: 0.4-1.9)$ \\
& P value MLR & 0.79 \\
\hline
\end{tabular}


Table A4 Sensitivity analyses

\begin{tabular}{|c|c|c|c|c|}
\hline Severity & & NOD1: * GG vs TT & & $T L R 2:{ }^{*} \mathrm{~A}$ vs $\mathrm{GG}$ \\
\hline Cohorts & OR crude & $2.1(95 \% \mathrm{Cl}: 1.1-4.1)$ & Cohort 2 OR crude & 3.2 (95\%Cl: $0.7-13.4)$ \\
\hline \multirow[t]{3}{*}{1 (selection), 2} & $P$ value crude & $0.03^{* *}$ & $P$ value crude & 0.10 \\
\hline & OR MLR & 2.0 (95\%Cl: $1.0-4.0)$ & OR MLR & 3.1 (95\%Cl: $0.7-13.1)$ \\
\hline & $P$ value MPL & $0.04^{* *}$ & $P$ value MPL & 0.13 \\
\hline \multirow[t]{4}{*}{ Cohort 5} & OR crude & $1.1(95 \% \mathrm{Cl}: 0.5-2.1)$ & & \\
\hline & $P$ value crude & 0.87 & & \\
\hline & OR MLR & 1.0 (95\%Cl: 0.5-2.0) & & \\
\hline & $P$ value MLR & 0.91 & & \\
\hline
\end{tabular}

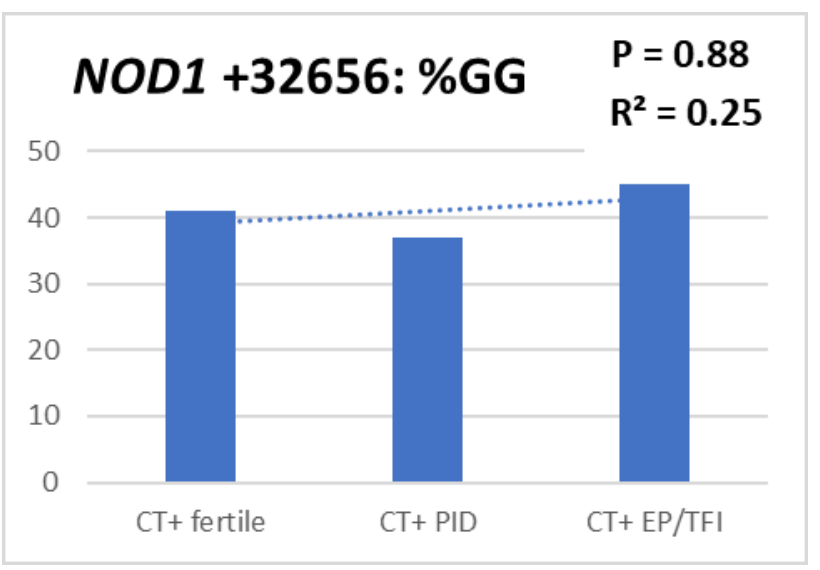

Figure A1: Sensitivity trend analysis using cohort (2) and a selection of cohort (1) Comparing genotype distributions between women based on increased severity: (1) Fertile CT positive women. (2) CT positive women with PID. 3. CT positive women with ectopic pregnancy and/ or tubal factor infertility. Percentage of genotypes as part of total. Abbreviations: $\quad \mathrm{CT}+, \quad$ Chlamydia trachomatis positive; PID, pelvic inflammatory disease; EP, ectopic pregnancy; TFI, tubal factor infertility. 


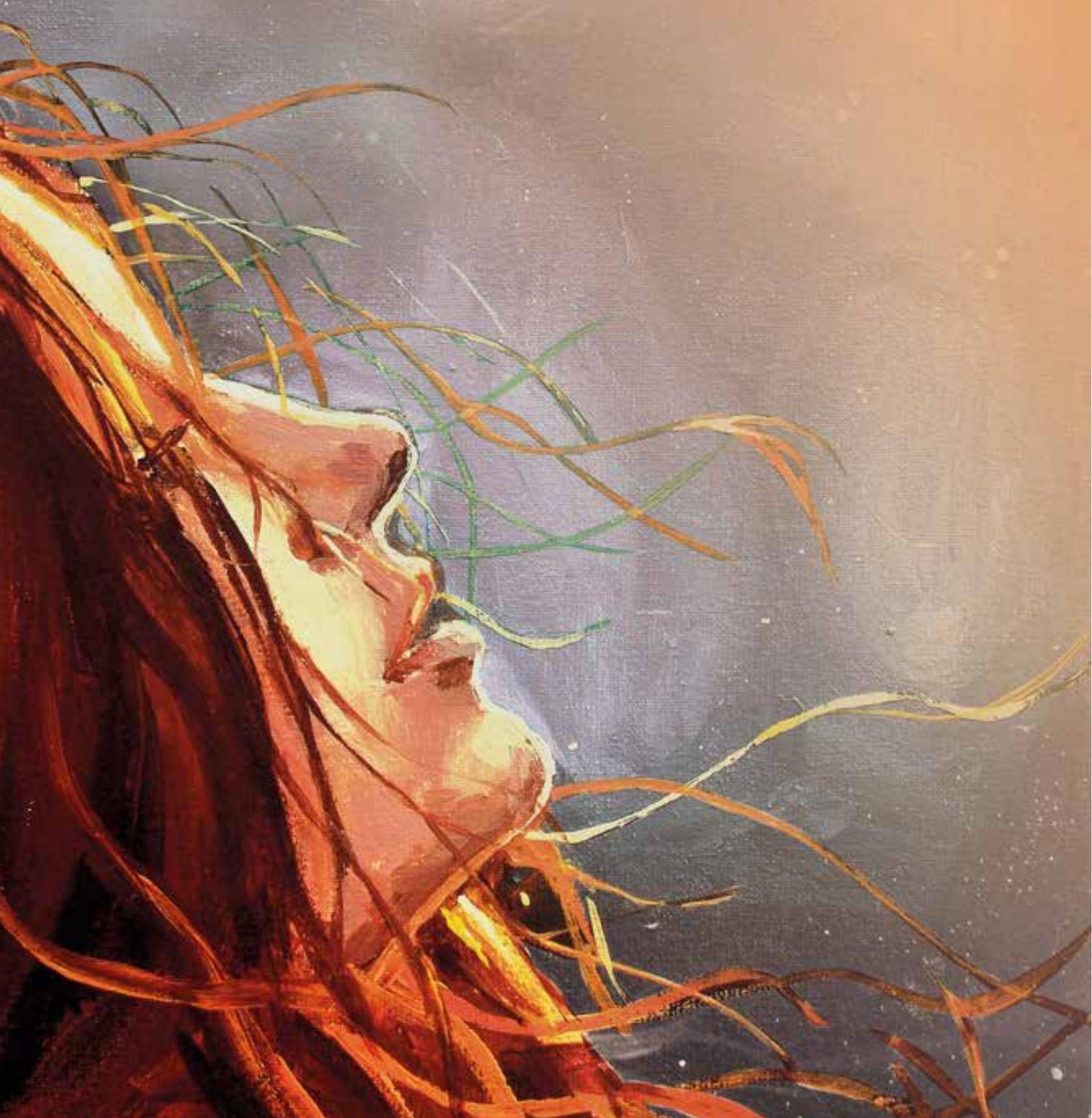




\section{Part 2}

The value of Chlamydia trachomatis serology 


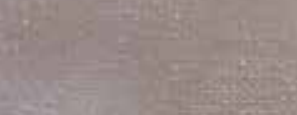

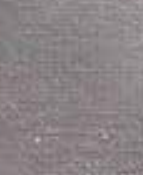

\section{sisting}

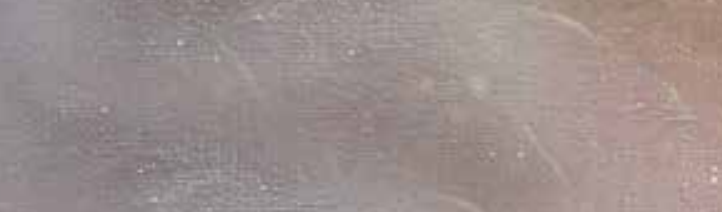

जince

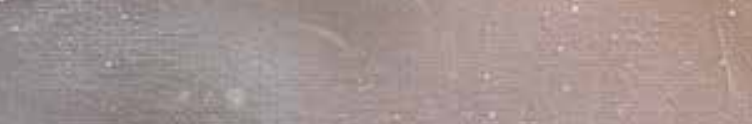

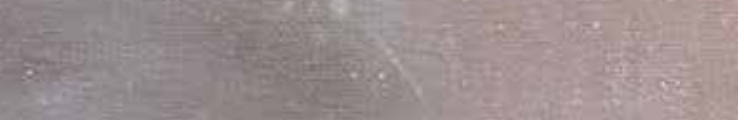

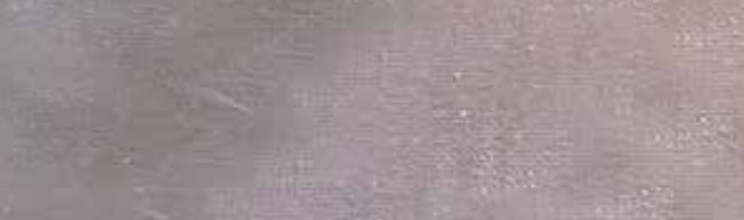

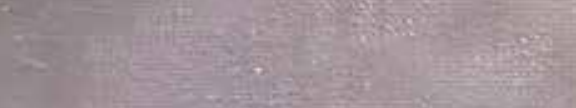

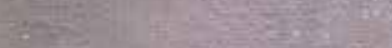

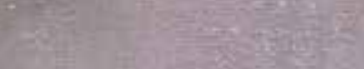

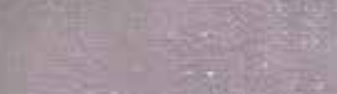

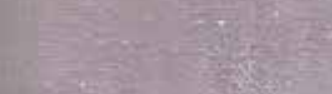

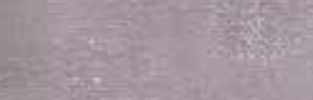

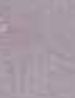

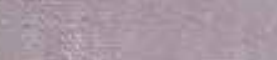

netwo

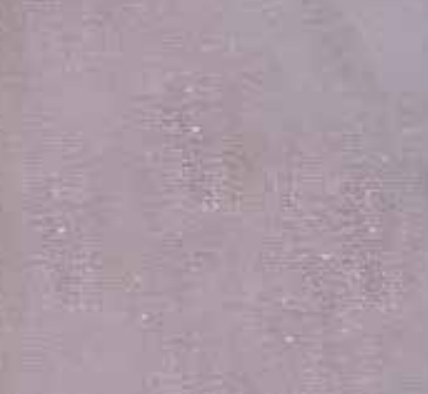

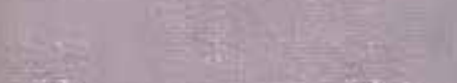

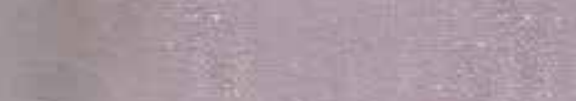




\section{Antibody Testing in Estimating Past Exposure to Chlamydia trachomatis in the Netherlands Chlamydia Cohort Study}

Bernice M Hoenderboom, Michelle E van Willige, Jolande A Land, Jolein Pleijster, Hannelore M Götz, Jan EAM van Bergen, Nicole HTM Dukers-Muijrers,

Christian JPA Hoebe, Birgit HB van Benthem, Servaas A Morré

Published in: Microorganisms 2019, 10.3390/microorganisms7100442 


\section{Abstract}

The asymptomatic course of Chlamydia trachomatis (CT) infections can result in underestimated CT lifetime prevalence. Antibody testing might improve this estimate. We assessed CT antibody positivity and predictive factors thereof in the Netherlands Chlamydia Cohort Study. Women who had $\geq 1$ CT Nucleic Acid Amplification Test (NAAT) in the study (2008-2011) and who provided self-reported information on NAATs were tested for CT major outer membrane protein specific IgG in serum (2016). CT antibody positivity was assessed and predictive factors were identified using multivariable logistic regressions, separately for CT-positive women ( $\geq 1$ positive NAAT or $\geq 1$ self-reported positive CT test) and CT-negative women (negative by study NAAT and self-report). Of the 3,613 women studied, 833 (23.1\%) were CT -positive. Among the CT-negative women, 208 (7.5\%, 95\% Cl 6.5-8.5) tested positive for CT antibodies. This increased CT lifetime prevalence with $5.8 \%$ (95\% Cl 5.0-6.5). Among women with a CT-positive history, 338 (40.6\%, 95\%Cl 38.5-44.1) tested positive. Predictive factors for antibody positivity related to lower social economic status, sexual risk behavior, multiple infections, higher body mass index, and non-smoking. CT antibody testing significantly increased the lifetime prevalence. Combining NAAT outcomes, self-reported positive tests, and antibody testing reduced misclassification in CT prevalence estimates. 


\section{Introduction}

Disease monitoring and proper evaluation of Chlamydia trachomatis (CT) control efforts are important and require accurate estimates of current and lifetime CT prevalence [1]. This is, however, challenging given the asymptomatic nature of the CT infection. Current prevalence estimates and our understanding of CT related complications are primarily based on studies that measure current (vaginal) infections by using nucleic acid amplification tests (NAATs) $[2,3]$. These tests have a high sensitivity to detect CT [4]. However, CT infections are asymptomatic in up to $70 \%$ of the cases in women [5]. Therefore, a considerable part of these infections might go unnoticed and thus remain undetected [2,6]. Presumably, an underestimation of the CT lifetime prevalence is the result $[6,7]$. Furthermore, estimating the proportion of infected women that later experiences CT related complications is difficult $[8,9]$.

Given these uncertainties, several studies proposed the use of CT antibody testing as an additional means to more accurately determine CT lifetime prevalence $[1,3,10]$. Elevated CT IgG levels in serum are a marker of a previous CT infection and can provide information on past infection $[3,11]$. Undetected and cleared infections can be included in lifetime prevalence estimates and improve the accuracy in either CT surveillance or in cohort studies assessing the effects of CT infections. However, the interpretation of CT seropositivity is not straightforward because not all women with CT infections will develop specific antibodies, furthermore, there is poor insight into the course of antibody titers, during and after CT infection, and individual variation thereof $[1,12,13]$. Characteristics of women with and without a positive CT NAAT test who are CT seropositive are not well-established $[3,14]$. We wanted to gain more insight into the benefit of CT antibody testing in estimating CT lifetime prevalence. First, we aimed to assess and compare CT antibody positivity among women with a CT-positive history (i.e., NAAT-positive results or self-reported positive test results) and in women with a negative history (NAAT-negative and no self-reported positive test). Second, we aimed to identify the predictive factors of CT antibody positivity in the two groups.

\section{Materials and Methods}

\section{Study Design and Population}

We used cross-sectional data from the Netherlands Chlamydia Cohort Study (NECCST), an ongoing longitudinal cohort study of women of reproductive age in the Netherlands, prospectively followed for $\geq 10$ years to investigate disease progression of CT [15]. NECCST is a follow-up study from the Chlamydia Screening Implementation (CSI) study, see Figure 1. In CSI, women were tested annually for CT through vaginal swabs or urine samples between 2008 and 2011 [16]. Women who participated in at least one round of CSI were recruited for NECCST in 2015-2016. All participants had $\geq 1$ NAAT test for CT during CSI, referred to as CSI-NAAT. Between 2008-2011 (CSI) and 2015-2016 (NECCST), women completed questionnaires about previous CT tests (and results), and on demographics, health characteristics, and (sexual) risk behavior. At the start of NECCST, 
capillary blood samples were collected to test for CT IgG antibodies. Women were included in the present study in case there was a negative or positive CT IgG antibody result available.

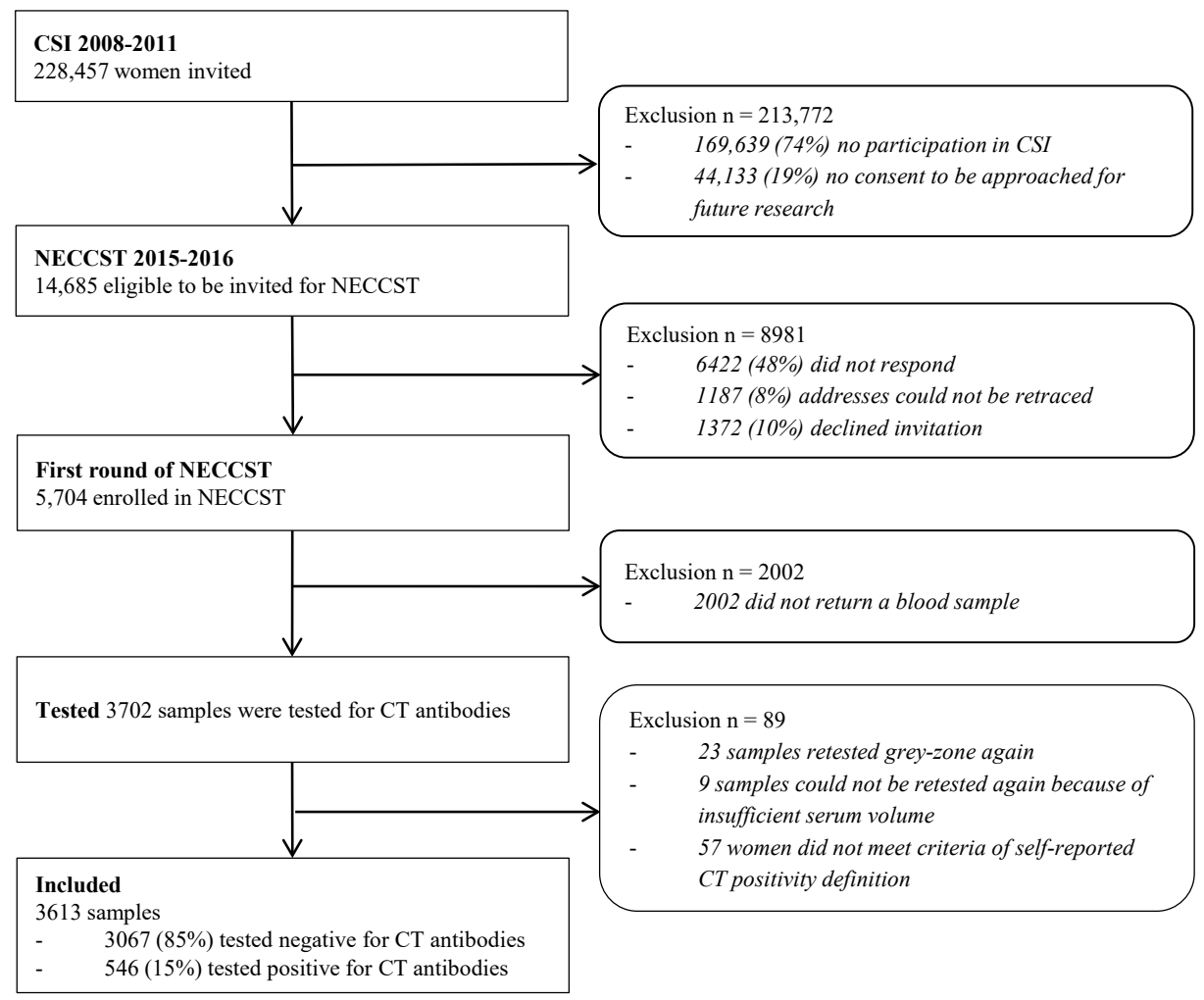

Figure 1. Flowchart for inclusion of participants in the present study, based on previous participation in the Chlamydia Screening Implementation (CSI) $(n=58,818)$ and Netherlands Chlamydia Cohort Study (NECCST) $(n=5704)$ projects.

\section{CT Antibody Testing}

Within two weeks after completing the initial NECCST questionnaire, participants received a kit at home for self-collection of capillary blood for the CT IgG antibody testing. Capillary blood was obtained via finger prick and collected in a collection tube (BD Microtainer ${ }^{\circ}$ serum separator tube, Becton, Dickinson and Company, USA). Samples were sent by regular mail to the laboratory and were immediately processed and centrifuged, in order to collect the serum. Subsequently, serum samples were stored at $-20^{\circ} \mathrm{C}$ until the serological measurement was performed [17]. To test for CT antibodies, the commercially available CT IgG ELISA plus (CT IgG ELISA plus; Medac, Wedel, Germany) test was chosen because this assay is the most widely used serology test in the Netherlands, which utilizes comparability between studies. In addition, this assay is 
used in clinics as part of infertility diagnostics. It is a peptide-based assay and used major outer membrane protein (MOMP) as the antigen of CT. The synthetic MOMP peptide is based on all available genetic information of the CT serovar, such that they detect all CT serovars without cross reactivity to other Chlamydiae, including Chlamydia pneumoniae [18]. This resulted in a high specificity of $97 \%$. Other advantages of the ELISA plus are the high-throughput and quantitative outcomes [18]. Sensitivity varied between $71 \%-93 \%$ in comparison to the microimmunofluorescence (MIF) or other serological tests, and between 30\%-77\% compared to the NAAT-positive women, depending on time since infection $[11,18,19]$. The assay was performed according to the manufacturer's instructions to test for the presence of CT IgG antibodies.

Quantitative serological outcomes were classified as negative (lgG concentration $<22 \mathrm{AU} / \mathrm{ml}$ ), grey-zone (IgG concentration 22-28 AU/ml), or positive (lgG concentration $\geq 28 \mathrm{AU} / \mathrm{ml}$ ). Samples with a grey-zone serological outcome were retested and the retest outcome was used. In cases where the samples tested in the grey-zone again or the samples could not be retested, the participants were excluded from this study (Figure 1, study flowchart).

\section{Nucleic Acid Amplification-Based Diagnostics}

During the annual CSI, CT tests were performed in self-collected vaginal swabs or urine samples. These samples were tested by NAATs, which are considered to be the preferred method for diagnosis of CT infections [4]. In CSI, there was a minimum of one and a maximum of four NAAT outcomes for all women, depending on participation.

\section{Questionnaires}

Potential predictive factors were obtained by CSI questionnaires and the initial NECCST questionnaire. CSI questionnaires addressed previous STIs, demographics, and sexual risk behavior. The NECCST questionnaire contained questions related to CT infections, sexual health, sexual risk behavior, and health characteristics. In case of at least one self-reported CT infection, the year of the positive CT test and the CT-related symptoms, such as vaginal discharge, abdominal pain, or pain during intercourse or intermenstrual vaginal bleeding, were documented. We used CSI data where possible in order to shorten the recall distance and to verify data.

\section{CT History}

A CT-positive history was determined by positive NAAT outcomes during CSI and self-reported positive CT tests during CSI or NECCST. A self-reported test was defined as a positive CT test (NAAT) performed in an STI clinic or hospital, by a GP, or performed in samples collected at home but tested in a laboratory. A CT-negative history was defined as no positive NAAT result and no self-reported positive CT tests.

\section{Potential Predictive Factors}

First, demographics were taken into account. Age of participants was assessed at the time of the initial NECCST questionnaire. Migration background was classified as 'Western' if both parents had a Western country of birth (i.e., a country from Europe [excluding Turkey], North-America, 
Oceania, Indonesia, and Japan), 'non-Western' if at least one parent had a non-Western country of birth, and 'unknown' if one parent was Western whereas the country of birth of the other parent was unknown, or when country of birth of both parents was unknown. Educational level was categorized by low- and middle-educational level versus high-educational level (low/medium —no education, primary education only, lower general secondary education, and vocational education, high-higher professional education and university education). Other potential predictive factors were - the number of CT infections, number of CT tests, years since last infection until the year of the initial NECCST questionnaire (serum test), CT symptoms (yes/no), and previous gonorrhea infections. Factors related to sexual risk behavior were-the number of lifetime sex partners, condom use with a casual partner, condom use with a steady partner, and age of sexual debut. Last, the following health characteristics were considered—smoking and Body Mass Index (BMI).

\section{Statistical Analysis}

Prior to conducting analyses, the amount of missing data was investigated. In addition, it was determined whether the missing data were dependent on the participants' characteristics or the outcome variable. Descriptive analyses were performed and characteristics of the total study population are presented. To compare characteristics between women with a positive and negative chlamydia history, the chi-squared test, $t$-tests, and the Wilcoxon rank-sum test were used.

CT antibody status was described and compared by a CT-negative history and a CT-positive history, using the chi-squared tests. The latter was further divided between CSI-NAAT positive women and women with self-reported positive CT tests only. The additional value of the CT antibody testing for estimating CT lifetime prevalence in combination with CT-NAAT and self-reported positive tests was calculated. Differences in CT lifetime prevalence's were tested with the McNemar test. To examine predictive factors of CT antibody positivity, multivariable logistic regression analyses were performed. The analyses were stratified by CT history (a negative history or a positive history). Univariable analyses tested associations between potential predictive factors and CT IgG antibody presence. $p$-values $<0.10$ were used as cut-off for inclusion in the multivariable model. In the multivariable model, a backward stepwise selection was used in order to manually remove factors that were not significantly $(p \geq 0.05)$ associated with CT antibody positivity anymore. Results of univariable and multivariable analyses were presented as odds ratios (ORs) and adjusted odds ratios (aORs) with 95\% confidence intervals (Cls). Statistical analyses were performed using STATA (Version 15.1; StataCorp, College Station, TX, USA).

\section{Ethics Approval}

This study was approved by the Medical Ethical Committee VU medical Center, Amsterdam the Netherlands (NL 51553.094.14/2015.903(A2019.336)). Date of approval 10/13/2015. 


\section{Results}

In total, 3,702 blood samples were tested for CT antibodies from women who had an available NAAT result and who completed the initial NECCST questionnaire. Of the 80 grey-zone samples, 32 were excluded from analyses because of insufficient serum volume for retesting or a second grey-zone test outcome. Additionally, 57 women were excluded because they did not meet the criteria for CT history positivity resulting in 3,613 women being included in the analyses; see Figure 1 . The amount of missing data was $0.5 \%$. Missing values did not depend on participant characteristics and were assumed to be at random.

\section{Participant Characteristics}

Table 1 summarizes the main characteristics of all participants, $n=3,613$. In total, 2,780 (76.9\%) participants had a CT-negative history and 833 (23.1\%) participants had a CT-positive history. Of participants with a CT-positive history, 206 (24.7\%) had $\geq 1$ positive CSI-NAAT result and 627 (75.3\%) were based on self-reported positive CT tests only. Participants predominantly had a Western migration background (83.4\%), were often highly educated (81.6\%), and the mean age was 31.2 (SD 3.8, range 21-38) years in 2015/2016 at the time of CT IgG antibody test. 


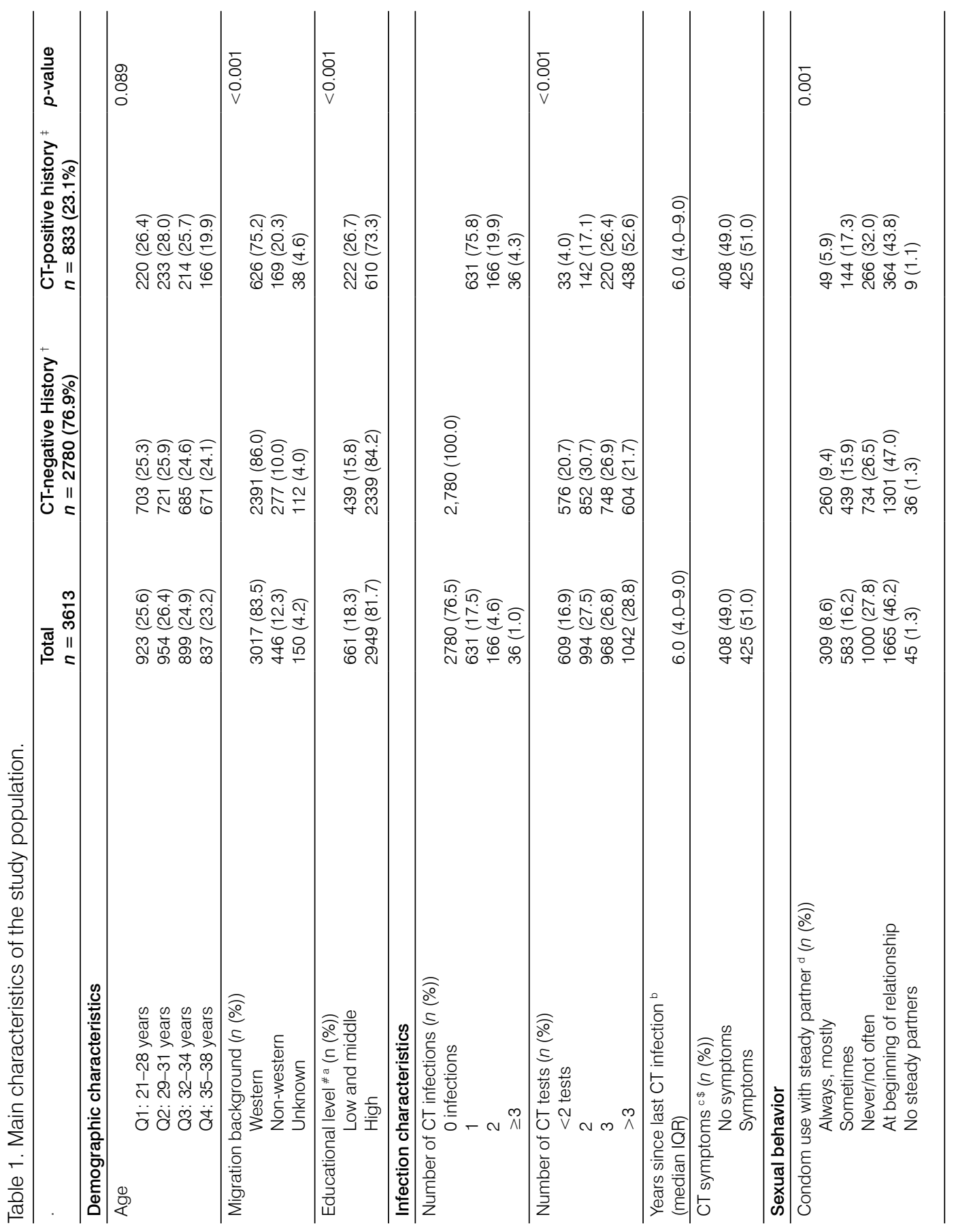




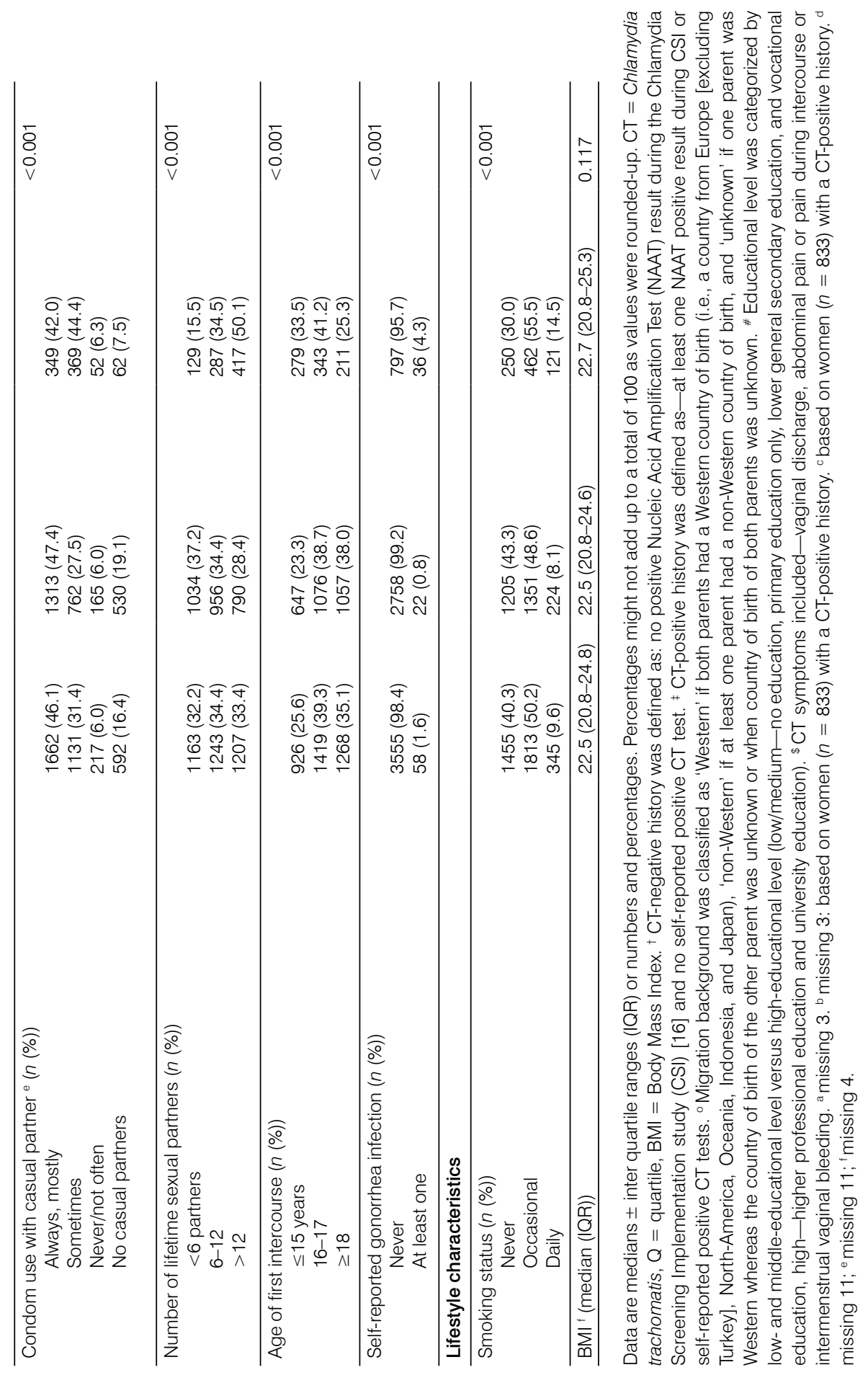




\section{CT Antibody Status}

Table 2 presents CT antibody status stratified by CT history, with a further distinction between participants with at least one CSI-NAAT positive result and participants with only self-reported positive CT tests. Of the 2,780 participants with a CT-negative history, 208 , i.e., $7.5 \%(95 \% \mathrm{CI}$ 6.5-8.5) tested positive for CT IgG, and among the 833 participants with a CT-positive history, 338, 40.6\% (95\% Cl 37.2-43.9) tested positive for CT IgG $(p<0.001)$. CSI-NAAT positives were more often ( $n=98,47.6 \%, 95 \% \mathrm{Cl} 40.6-54.6)$ CT-lgG-positive, compared to women with only self-reported positive tests ( $n=240,38.3 \%, 95 \% \mathrm{Cl} 34.5-42.1), p=0.018$.

Including outcomes of the CT antibody tests in the CT history definition (i.e., combining CSI-NAAT positive tests $(n=206)$, only the self-reported positive tests $(n=627)$, and only the CT antibody positives $(n=208)$ increased the lifetime CT prevalence from $23.1 \%(95 \% \mathrm{Cl} 21.7-24.5)$ to $28.8 \%$ (95\% Cl 27.3-30.3). This was a significant increase of 5.8\% (95\% Cl 5.0-6.5), $p<0.001$.

Table 2. CT IgG antibody status in women with a CT-positive history and a CT-negative history.

\begin{tabular}{rlll}
\hline & CT IgG Positive & Total & $p$ value \\
\hline CT-negative history $^{\dagger}$ & $208(7.5 \%)$ & $2,780(100 \%)$ & $<0.001^{*}$ \\
CT-positive history $^{\ddagger}$ & $338(40.6 \%)$ & $833(100 \%)$ & \\
NAAT positive in CSI & $98(47.6 \%)$ & & $0.018^{* *}$ \\
Self-reported positive & $240(38.3 \%)$ & & \\
Total & $546(15.1 \%)$ & $3,613(100 \%)$ & \\
\hline
\end{tabular}

CT_Chlamydia trachomatis, IgG-Immunoglobulin G, NAAT_Nucleic Acid Amplification Test, CSIChlamydia Screening Implementation study. ${ }^{\dagger}$ CT-negative history was defined as: no positive NAAT result during the CSI [16] and no self-reported positive CT test. ${ }^{~ C T-p o s i t i v e ~ h i s t o r y ~ w a s ~ d e f i n e d ~ a s: ~ a t ~ l e a s t ~}$ one NAAT positive result during CSI or self-reported positive CT test. * $p$ value of the difference in CT IgG positivity between CT history negative and CT history positive women. ${ }^{*} p$-value of the difference in CT IgG positivity between CSI-NAAT positives and self-reported positive tests only.

\section{Predictors of Having CT Antibodies}

Table 3 shows univariable associations with CT antibodies and predictive factors of CT antibody positivity, identified by multivariable analyses. For women with a CT-positive history, multivariable analysis indicated that only the following factors remains associated with CT antibody positivity: unknown migration background, higher number of CT infections, and non-smoking.

For women with a CT-negative history, multivariable analysis demonstrated that the predictive factors of CT antibody positivity were non-Western or unknown migration background, low/middleeducational level, higher number of lifetime sexual partners, younger age at first intercourse, and $\mathrm{BMI}>30$. 


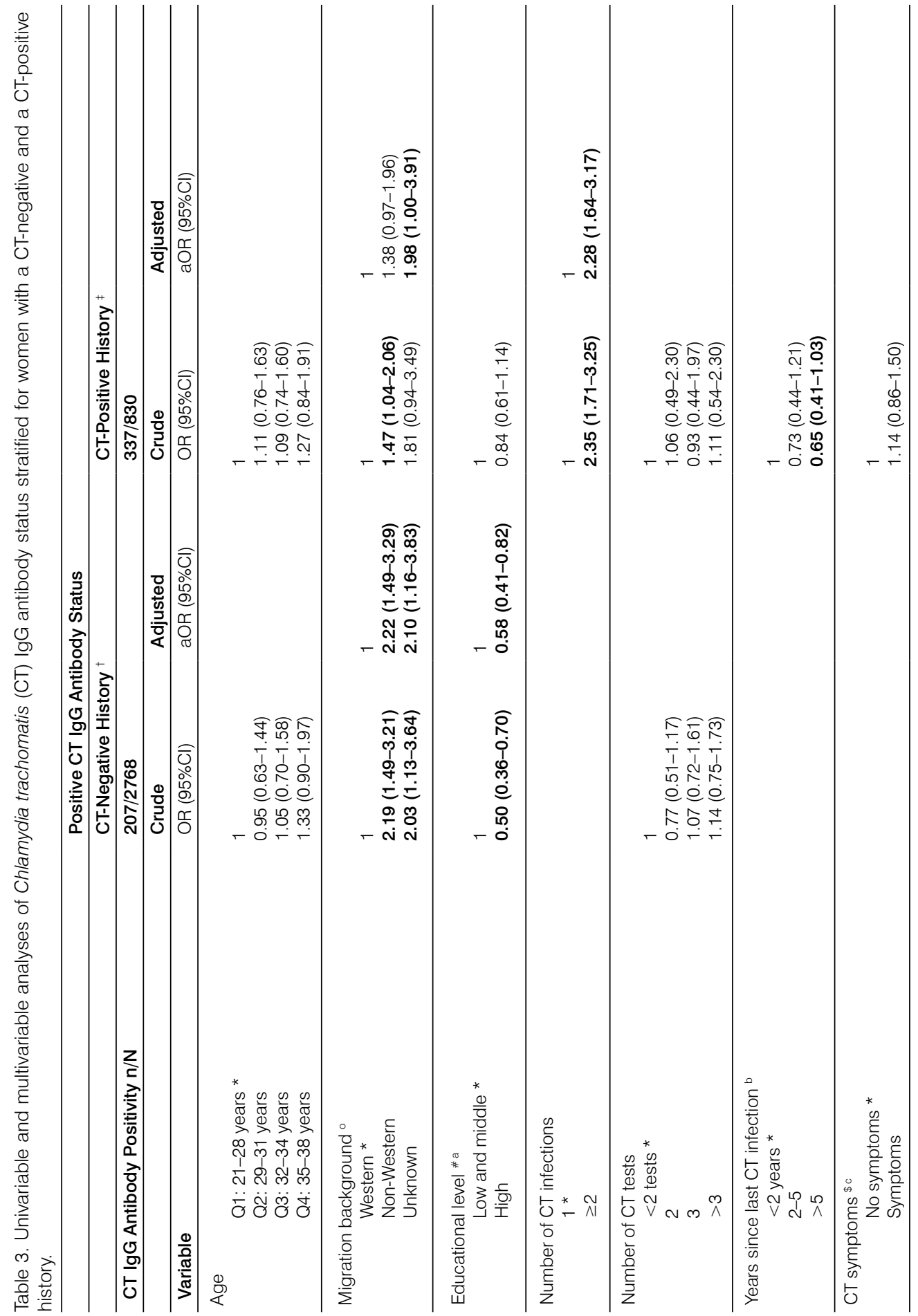




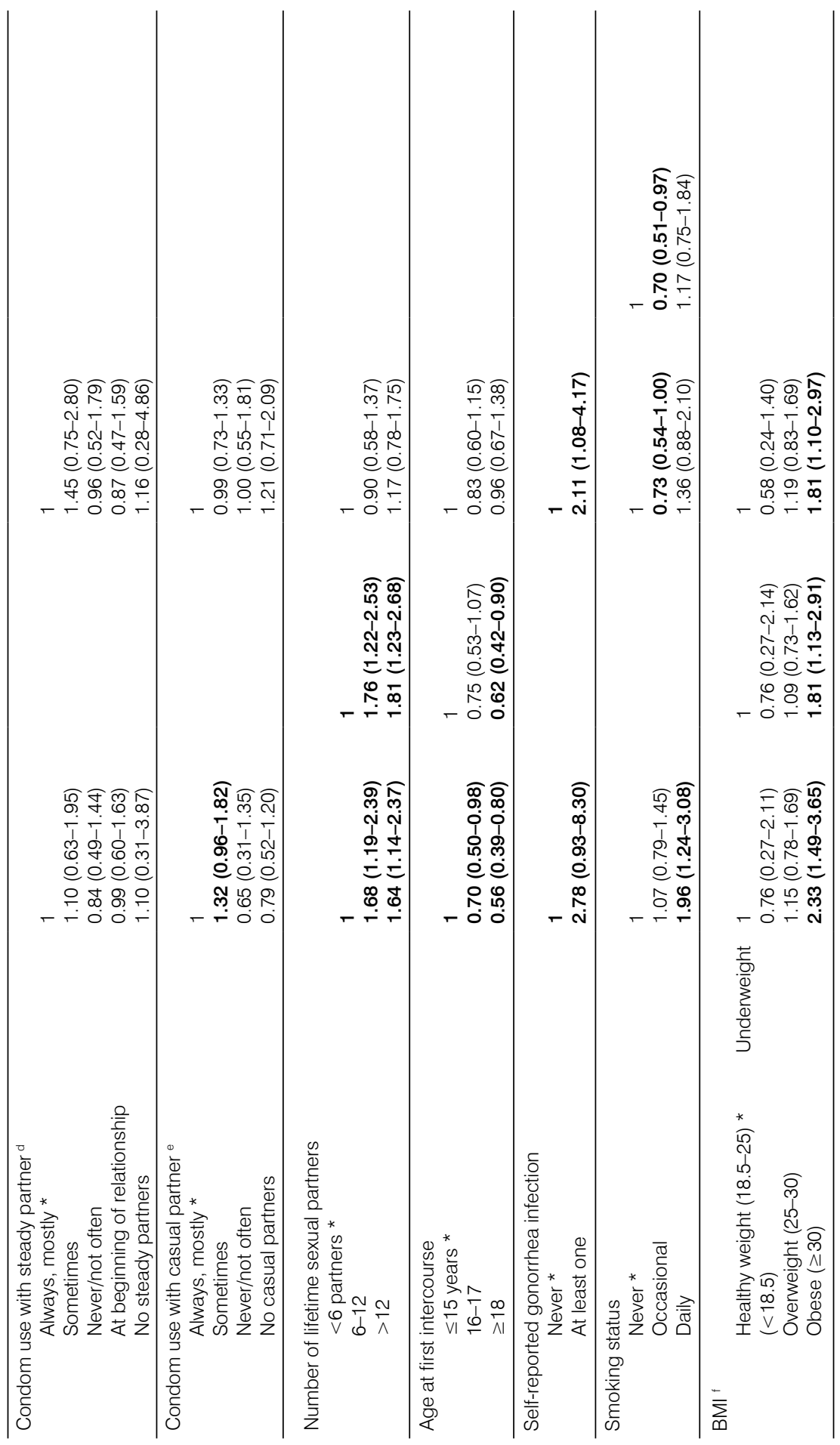

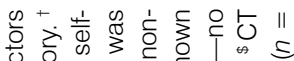

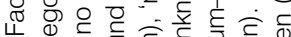
$\times$ 정뭉

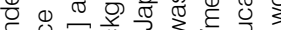

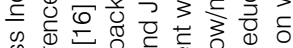

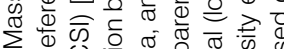

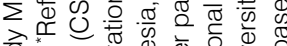

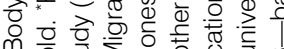

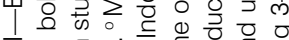

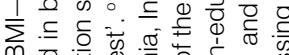

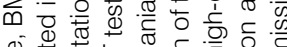

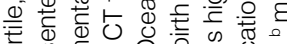
宁 क ह

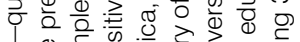

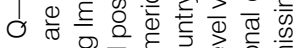

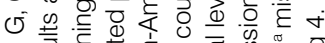

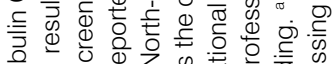

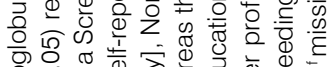

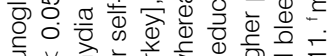
है $\vee$ है

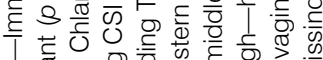
ग)

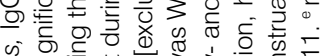
心) है $\frac{\dot{\omega}}{\omega}$ क गे $\frac{0}{0}$ की

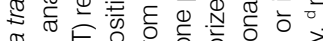

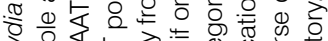

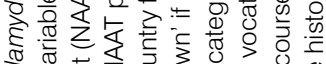

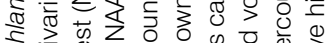
Uิ

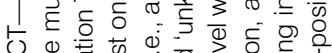

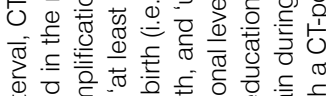

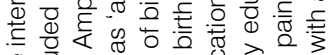

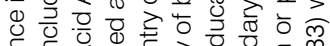
ब. 更

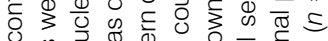

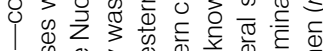

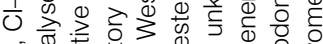

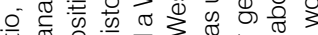
స్ర

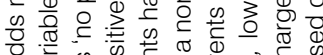
व

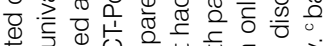

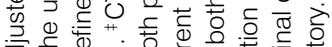

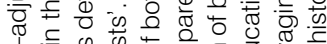

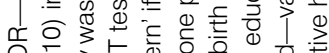

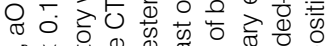

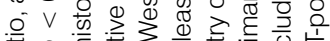

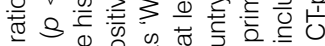

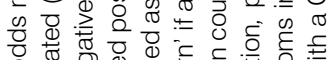

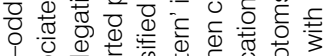

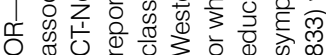




\section{Discussion}

In this cohort of women of reproductive age, eight percent of women who previously tested negative for CT by NAAT, tested positive for CT IgG antibodies. This increased the CT lifetime prevalence estimate with six percent. Among women who previously tested positive for CT by NAAT, 40\% tested positive for the CT IgG antibodies. In women with a CT-positive history (CSINAAT positive or self-reported positive CT tests), the predictive factors of CT antibody positivity were an unknown migration background and multiple CT infections, while occasional smoking (compared to current smoking) had a preventive effect. In women with a CT-negative history, predictive factors of previous unnoticed infections were a non-Western and unknown migration background, low- or middle-educational level, higher number of lifetime sexual partners, younger age at first sexual intercourse, and obesity.

CT IgG antibody testing identified 208 unnoticed CT infections among 2,780 women; infections that otherwise would have been missed. Therefore, in this population of women with a mean age of 31 (SD 4), lifetime CT prevalence increased by six percent from 23\% (i.e., CSI-NAAT positive or self-reported positive tests) to $29 \%$, including CT antibody testing. Although an increase of six percent suggests only a minor increase, the 208 additional CT history positives accounted for twenty percent $(208 / 1,041)$ of all CT history positives. This was similar for CSI-NAAT positives that also accounted for $20 \%(206 / 1,041)$ of CT history positives, whereas the contribution from self-reported positive CT tests was $60 \%$. Focusing only on NAAT and self-reported tests has its limitations. Although NAATs are highly sensitive and specific [4], only current CT infections can be measured. Median number of NAAT tests per participant in CSI was two. Including only NAAT outcomes for the CT lifetime prevalence would result in a large underestimation. Self-reported positive tests contributed the most in CT lifetime prevalence estimates, but might have induced recall bias and resulted in misclassifications in CT history. This was demonstrated in the difference in CT antibodies among women with a CSI-NAAT positive result (48\% CT IgG positive) and women who only had a self-reported positive test (38\% CT IgG positive). Therefore, combining NAAT outcomes, self-reported positive test outcomes, and CT antibody test outcomes results in more robust prevalence estimates. This would not erase misclassification but would reduce it. Consequently, estimation of the proportion of women who experiences CT-related complications, for example in cohort studies, would be more precise by including lgG antibody testing because there would be less misclassification in CT status [1,20].

Nevertheless, among women with a positive NAAT, less than half tested positive for CT IgG antibodies. Therefore, the proportion of women with past CT infections is presumably higher. Among CT- positive history women, $60 \%$ of infections were missed while using antibody testing. Assuming a similar test sensitivity in both the CT- positive and CT-negative group, and by applying this percentage missed to the CT- negative group, one could hypothesize that true CT (sero-) positivity is around $20 \%$ instead of $8 \%$ in the CT- negative group. However, sensitivity might differ between these two groups as in the CT- positive group, 51\% of women reported symptoms and $24 \%$ of women had multiple CT infections, which could have caused higher antibody responses $[11,21]$. 
In participants with a CT-positive history, an unknown migration background and multiple CT infections were strongly associated with CT antibody positivity. This provides additional evidence that repeated exposure to CT infections is an important predictor of CT antibody positivity $[11,22]$. Occasional smoking compared to non-smoking was demonstrated to be a condition that decreases the likelihood of having CT IgG antibodies. We only found this association among women who previously tested positive for CT. It is, therefore, not a risk or protective factor for acquiring a CT infection, but specifically in having CT IgG antibodies following CT infection. The association might be explained by alterations in the immune response, such as an accelerated decrease of antibodies over time or weaker antibody responses. It is known that smoking can downregulate the immune system [23]. However, we did not find a significant difference with current smokers as compared to non-smokers.

In participants with a CT-negative history, predictive factors for antibody positivity could be interpreted as predictive factors for women with unnoticed infections next to predictors for seroconversion. A non-Western or unknown migration background and low/middle-educational level were predictive for antibody positivity $[24,25]$ and both characteristics related to a lower socioeconomic status (SES), which was associated with increased sexual risk behavior [26]. Additionally, previous research found higher CT antibody responses among African-Americans compared to Caucasians [13]. A positive association was found between a high number of lifetime sexual partners and CT antibody positivity, although this association was less strong than demonstrated in a previous study [10]. Age at time of antibody test was not a risk factor for having antibodies, probably because the majority of women in the cohort were all around thirty years of age. However, younger age at first sexual intercourse also increased the risk of being CT antibody positive and was consistent with previous research $[10,27]$. Young age is a consistent risk factor for CT acquisition, which could suggest an acquired and protective immune response [28]. Moreover, women with obesity had an increased risk of being CT antibody positive. We do not have a clear explanation for this finding. Although obesity is a condition that affects those with a low SES more often [29], the association remained after correcting for education level and migration status in the multivariable model. This suggests that a lower SES is not an explanation for this finding. On the other hand, we cannot completely rule out residual confounding. It might be that $\mathrm{BMI}$ is not completely explained by the variables we have in the dataset and that $\mathrm{BMI}$ is still linked to a lower SES and, therefore, poses a higher risk for acquiring CT infection and not specifically CT IgG antibodies. Especially because obesity is associated with impaired immunity and reduced immunocompetence [30], we would expect to find a decreased risk for CT antibodies instead of an increased risk. It would be interesting to study a possible association between CT infection or presence of CT antibodies and BMI in other cohorts. Last, participants with a CTnegative history and a positive CT antibody test presumably did not receive treatment for the infection. This IgG test does not give any information on whether the infection was past or current. Hence, treatment is not indicated, which is in line with national and international guidelines [31]. Several limitations should be noted. The antibody test specificity (although high-97\%) could, in combination with the low seroprevalence in the CT- negative group, result in a considerable number of false positives. However, the specificity issues reported in general in the past based on 
the potential cross reactivity with Chlamydia pneumoniae, Chlamydia psittaci, and Acinetobacter were not applicable in the current peptide-based ELISA from Medac in which a small peptide from the MOMP protein was selected with no cross reactivity with $C$. pneumoniae, in contrast to either lipopolysaccharide-based ELISAs or total MOMP-based ones [32-34]. Furthermore, there was the possibility of selection bias. The overall CT antibody positivity rate of $16 \%$ found in this study was higher than that found in a population-based seroprevalence study among 25-39-year old women, which was previously conducted in the Netherlands; a seroprevalence of $9 \%$ in 2007 was found [14]. A possible explanation might be that NECCST participants were at higher risk for CT when compared to the general population who participated in the population-based study. This was also seen in the screening trial. CSI participants had a higher CT positivity rate compared to the estimated population prevalence and had higher behavioral risk factors compared to people not participating in the CSI, which suggest a self-selection for screening, based on personal risk perception $[16,35]$. This might influence the generalizability of the results. The $8 \%$ antibody positives in the CT-negative group could be an overestimation when compared to the general population. To gain more insight into the proportion of women with unnoticed previous CT infections and thereby improve the prevalence estimates even more, further research should be conducted. For example, assays based on different CT antigens such as the Pgp3 in combination with MOMP-based assays or assays using a range of CT antigens to determine the most reactive CT antigens might increase insight $[12,36,37]$. Additionally, the effect of time on antibody levels requires further study. In our multivariable analyses, time measured in years, since last infection was not a predictive factor for CT antibody positivity in multivariable analysis. According to a study by Horner et al. [11], antibodies were detected in only $38 \%$ of participants from genitourinary medicine clinics six months after infection, as compared to $77 \%$ at time of infection, using the ELISA plus from Medac $[19,21]$. This suggests that the antibody levels decreased within the first months after infection, but no significant further reduction happened after six months. This was comparable with a recent study in which the same assay was used, antibodies were measured within three months and three to ten years after infection; seroprevalence was $66 \%$ and $35 \%$, respectively [22].

In conclusion, we demonstrated that CT IgG antibody testing identified women with unnoticed CT infections among women previously tested negative for CT by NAAT. Although not all women tested positive for CT antibodies despite a previous CT infection, the extra infections found with antibody testing significantly increased the CT lifetime prevalence estimates. Consequently, this improved the estimates on proportions of women who experienced CT-related complications. In estimating the CT lifetime prevalence, we recommended CT antibody testing in combination with previous chlamydia test outcomes. 


\section{References}

1. Woodhall, S.C.; Gorwitz, R.J.; Migchelsen, S.J.; Gottlieb, S.L.; Horner, P.J.; Geisler, W.M.; Winstanley, C.; Hufnagel, K.; Waterboer T.; Martin, D.L.; et al. Advancing the Public Health Applications of Chlamydia Trachomatis Serology. Lancet Infect. Dis. 2018, 18, e399e407.

2. Jackson, L.J.; Auguste, P.; Low, N.; Roberts, T.E. Valuing the Health States Associated with Chlamydia Trachomatis Infections and Their Sequelae: A Systematic Review of Economic Evaluations and Primary Studies. Value Health 2014, 17, 116-130.

3. Johnson, A.M.; Horner, P. A New Role for Chlamydia Trachomatis Serology? Sex. Transm Infect. 2008, 84, 79-80.

4. Meyer, T. Diagnostic Procedures to Detect Chlamydia Trachomatis Infections. Microorganisms 2016, 4, 25.

5. Low, N.; McCarthy, A.; Macleod, J.; Salisbury, C.; Campbell, R.; Roberts, T.E.; Horner, P.; Skidmore, S.; Sterne, J.A.; Sanford, E.; et al. Epidemiological, Social, Diagnostic and Economic Evaluation of Population Screening for Genital Chlamydial Infection. Health Technol. Assess. 2007, 11, iii-iv, ix-xii, 1-165.

6. ECDC. Chlamydia control in Europe Literature review ECDC. In Stockholm: European Centre for Disease Prevention and Control; European Centre for Disease Prevention and Control: Stockholm, Sweden, 2014.

7. Miller, W.C.; Epidemiology of Chlamydial Infection: Are We Losing Ground? Sex. Transm Infect. 2008, 84, 82-86.

8. Haggerty, C.L.; Gottlieb, S.L.; Taylor, B.D.; Low, N.; Xu, F.; Ness, R.B. Risk of Sequelae After Chlamydia Trachomatis Genital Infection in women. J. Infect. Dis. 2010, 201 (Suppl. 2), S134-S155.

9. Wallace, L.A.; Scoular, A.; Hart, G.; Reid, M.; Wilson, P.; Goldberg, D.J. What is the Excess Risk of Infertility in Women After Genital Chlamydia Infection? A Systematic Review of the Evidence. Sex. Transm Infect. 2008, 84, 171-175.

10. Woodhall, S.C.; Wills, G.S.; Horner, P.J.; Craig, R.; Mindell, J.S.; Murphy, G.; McClure, M.O.; Soldan, K.; Nardone, A.; Johnson, A.M. Chlamydia Trachomatis Pgp3 Antibody Population Seroprevalence before and during an Era of Widespread Opportunistic Chlamydia Screening in England (1994-2012). PLOS ONE 2017, 12, e0152810

11. Horner, P.J.; Wills, G.S.; Reynolds, R.; Johnson, A.M.; Muir, D.A.; Winston, A.; Broadbent, A.J.; Parker, D.; McClure, M.O. Effect of Time Since Exposure to Chlamydia Trachomatis on Chlamydia Antibody Detection in Women: A Cross-Sectional Study. Sex. Transm Infect. 2013, 89, 398-403.

12. Wills, G.S.; Horner, P.J.; Reynolds, R.; Johnson, A.M.; Muir, D.A.; Brown, D.W.; Winston, A.; Broadbent, A.J.; Parker, D.; McClure, M.O. Pgp3 Antibody Enzyme-Linked Immunosorbent Assay, a Sensitive and Specific Assay for Seroepidemiological Analysis of Chlamydia Trachomatis Infection. Clin. Vaccine Immunol. 2009, 16, 835-843.

13. Geisler, W.M.; Morrison, S.G.; Doemland, M.L.; lqbal, S.M.; Su, J.; Mancevski, A.; Hook, E.W., 3rd; Morrison, R.P. Immunoglobulin-Specific Responses to Chlamydia Elementary Bodies in Individuals with and at Risk for Genital Chlamydial Infection. J. Infect. Dis. 2012, 206, 1836-1843.

14. Van Aar, F.; De Moraes, M.; Morre, S.A.; Van Bergen, J.E.; Van Der Klis, F.R.; Land, J.A.; Van Der Sande, M.A.; Van Den Broek, I.V. Chlamydia Trachomatis IgG Seroprevalence in the General Population of the Netherlands in 1996 and in 2007: Differential Changes by Gender and Age. Sex. Transm Infect. 2014, 90, 434-440.

15. Hoenderboom, B.M.; Van Oeffelen, A.A.; Van Benthem, B.H.; Van Bergen, J.E.; DukersMuijrers, N.H.; Gotz, H.M.; Hoebe, C.J.; Hogewoning, A.A.; Van Der Klis, F.R.; Van 
Baarle, D.; et al. The Netherlands Chlamydia Cohort Study (NECCST) Protocol to Assess the Risk of Late Complications Following Chlamydia Trachomatis Infection in Women. BMC Infect. Dis. 2017, 17, 264.

16. Van Den Broek, I.V.F.; Van Bergen, J.E.A.M.; Brouwers, E.E.H.G.; Fennema, J.S.A.; Gotz, H.M.; Hoebe, C.J.P.A.; Koekenbier, R.H.; Kretzschmar, M.; Over, E.A.B.; Schmid, B.V.; et al. Effectiveness of Yearly, Register Based Screening for Chlamydia in the Netherlands: Controlled Trial with Randomised Stepped Wedge Implementation. BMJ Br. Med. J. 2012, $345, \mathrm{e} 4316$.

17. Hoenderboom, B.M.; Van Ess, E.F.; Van Den Broek, I.V.F.; Van Loo, I.H.M.; Hoebe, C.; Ouburg, S.; Morre, S.A. Chlamydia Trachomatis Antibody Detection in home-Collected Blood Samples for Use in Epidemiological Studies. J. Microbiol. Methods 2018, 144, 164-167.

18. Baud, D.; Regan, L.; Greub, G. Comparison of Five Commercial Serological Tests for the Detection of Anti-Chlamydia Trachomatis Antibodies. Eur. J. Clin. Microbiol Infect. Dis. 2010, 29, 669-675.

19. Morre, S.A.; Munk, C.; Persson, K.; KrugerKjaer, S.; Van Dijk, R.; Meijer, C.J.L.M.; Van Den Brule, A.J.C. Comparison of Three Commercially Available Peptide-Based Immunoglobulin $\mathrm{G}$ ( $\mathrm{IgG}$ ) and IgA Assays to Microimmunofluorescence Assay for Detection of Chlamydia Trachomatis Antibodies. J. Clin. Microbiol. 2002, 40, 584-587.

20. Price, M.J.; Ades, A.E.; Welton, N.J.; Macleod, J.; Turner, K.; Simms, I.; Horner, P.J. How Much Tubal Factor Infertility is Caused by Chlamydia? Estimates Based on Serological Evidence Corrected for Sensitivity and Specificity. Sex. Transm Dis. 2012, 39, 608-613.

21. Blomquist, P.B.; Mighelsen, S.J.; Wills, G.; McClure, E.; Ades, A.E.; Kounali, D.; Dunbar, J.K.; McClure, M.O.; Soldan, K.; Woodhall, S.C.; et al. Sera Selected from National STI Surveillance System Shows Chlamydia Trachomatis PgP3 Antibody Correlates with
Time Since Infection and Number of Previous Infections. PLOS ONE 2018, 13, e0208652.

22. Ohman, H.; Rantsi, T.; Joki-Korpela, P.; Tiitinen, A.; Surcel, H.M. Prevalence and Persistence of Chlamydia Trachomatis-Specific Antibodies after Occasional and Recurrent Infections. Sex. Transm Infect. 2019, doi:10.1136/ sextrans-2018-053915.

23. Qiu, F.; Liang, C.L.; Liu, H.; Zeng, Y.Q.; Hou, S.; Huang, S.; Lai, X.; Dai, Z. Impacts of Cigarette Smoking on Immune Responsiveness: Up and Down or Upside Down? Oncotarget 2017, 8 , 268-284.

24. Hulstein, S.H.; Matser, A.; Alberts, C.J.; Snijder, M.B.; Willhauck-Fleckenstein, M.; Hufnagel, K.; Prins, M.; De Vries, H.J.C.; Schim Van Der Loeff, M.F.; Waterboer, T. Differences in Chlamydia Trachomatis Seroprevalence between Ethnic Groups cannot be Fully Explained by Socioeconomic Status, Sexual Healthcare Seeking Behavior or Sexual Risk Behavior: A Cross-Sectional Analysis in the HEalthy Llfe in an Urban Setting (HELIUS) study. BMC. Infect. Dis. 2018, 18, 612.

25. Crichton, J.; Hickman, M.; Campbell, R.; Batista-Ferrer, H.; Macleod, J. Socioeconomic Factors and Other Sources of Variation in the Prevalence of Genital Chlamydia Infections: A Systematic Review and Meta-Analysis. BMC. Public Health 2015, 15, 729.

26. Kipping, R.R.; Smith, M.; Heron, J.; Hickman, M.; Campbell, R. Multiple Risk Behaviour in Adolescence and Socio-Economic Status: Findings from a UK Birth Cohort. Eur. J. Public Health 2015, 25, 44-49.

27. Horner, P.J.; Wills, G.S.; Righarts, A.; Vieira, S.; Kounali, D.; Samuel, D.; Winston, A.; Muir, D.; Dickson, N.P.; McClure, M.O. Chlamydia Trachomatis Pgp3 Antibody Persists and Correlates with Self-Reported Infection and Behavioural Risks in a Blinded Cohort Study. PLOS ONE 2016, 11, e0151497.

28. Rekart, M.L.; Brunham, R.C. Epidemiology of chlamydial infection: Are We Losing Ground? Sex. Transm Infect. 2008, 84, 87-91. 
29. Wang, Y.; Beydoun, M.A. The obesity epidemic in the United States-Gender, Age, Socioeconomic, Racial/Ethnic, and Geographic Characteristics: A Systematic Review and MetaRegression Analysis. Epidemiol. Rev. 2007, 29, 6-28.

30. Milner, J.J.; Beck, M.A. The Impact of Obesity on the Immune Response to Infection. Proc. Nutr. Soc. 2012, 71, 298-306.

31. Lanjouw, E.; Ouburg, S.; de Vries, H.J.; Stary, A.; Radcliffe, K.; Unemo, M. 2015 European Guideline on the Management of Chlamydia Trachomatis Infections. Int. J. STD AIDS 2016, 27, 333-348.

32. Forsey, T. Antibodies to Chlamydia Trachomatis. Genitourin Med. 1987, 63, 279.

33. Brade, H.; Brunner, H. Serological Cross- 37 Reactions between Acinetobacter Calcoaceticus and Chlamydiae. J. Clin. Microbiol 1979, 10, 819-822.

34. Land, J.A.; Gijsen, A.P.; Kessels, A.G.; Slobbe, M.E.; Bruggeman, C.A. Performance of Five Serological Chlamydia Antibody Tests in
Subfertile Women. Hum. Reprod. 2003, 18, 2621-2627.

35. Op De Coul, E.L.; Gotz, H.M.; Van Bergen, J.E.; Fennema, J.S.; Hoebe, C.J.; Koekenbier, R.H.; Pars, L.L.; Van Ravesteijn, S.M.; Van Der Sande, M.A.; Van Den Broek, I.V. Who Participates in the Dutch Chlamydia Screening? A Study on Demographic and Behavioral Correlates of Participation and Positivity. Sex. Transm Dis. 2012, 39, 97-103.

36. Wang, J.; Zhang, Y.; Lu, C.; Lei, L.; Yu, P.; Zhong, G. A Genome-Wide Profiling of the Humoral Immune Response to Chlamydia Trachomatis Infection Reveals Vaccine Candidate Antigens Expressed in Humans. J. Immunol. 2010, 185, 1670-1680.

7. Hufnagel, K.; Lueong, S.; WillhauckFleckenstein, M.; Hotz-Wagenblatt, A.; Miao, B.; Bauer, A.; Michel, A.; Butt, J.; Pawlita, M.; Hoheisel, J.D.; et al. Immunoprofiling of Chlamydia Trachomatis Using whole-Proteome Microarrays Generated by on-Chip in Situ Expression. Sci. Rep. 2018, 8, 7503. 



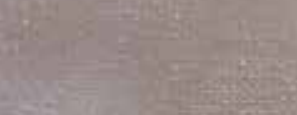

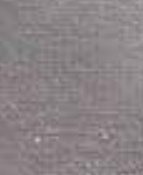

\section{sisting}

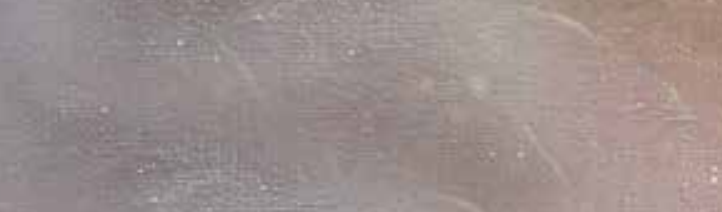

जince

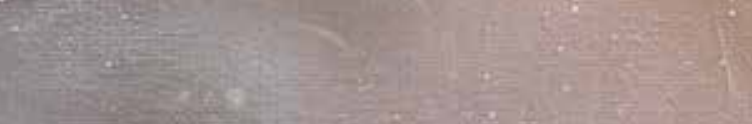

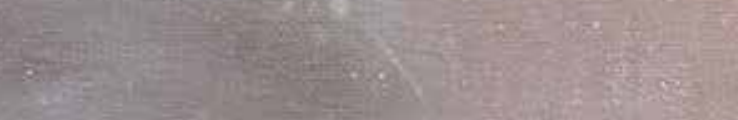

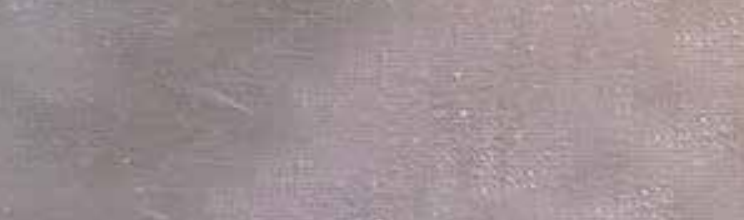

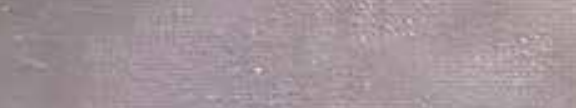

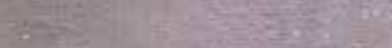

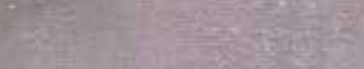

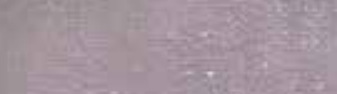

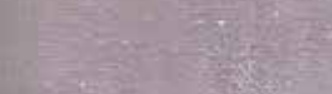

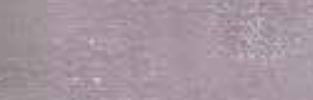

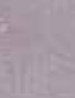

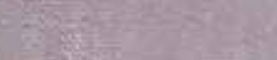

netwo

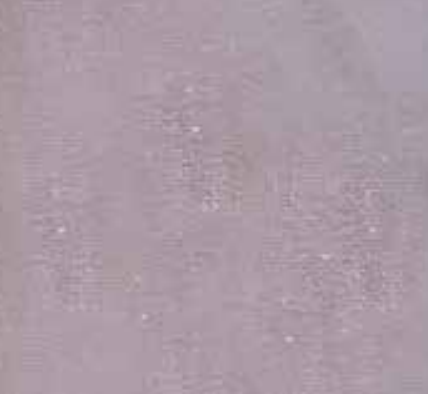

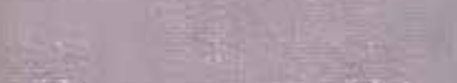

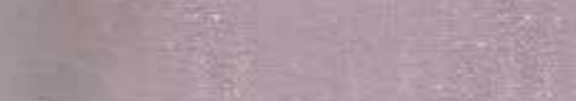




\section{6}

\section{Chlamydia trachomatis Whole-Proteome Microarray Analysis of the Netherlands Chlamydia Cohort Study}

Katrin Hufnagel*, Bernice M Hoenderboom*, Christoph Harmel, Juliane K Rohland Birgit HB van Benthem, Servaas A Morré, Tim Waterboer

*Shared first authorship

Published in: Microorganisms 2019, 10.3390/microorganisms7120703 


\section{Abstract}

Chlamydia trachomatis (Ct) whole-proteome microarrays were utilized to identify antibody patterns associated with infection; pelvic inflammatory disease (PID), tubal factor infertility, chronic pelvic pain (CPP) and ectopic pregnancy in a subsample of the Netherlands Chlamydia cohort study. Serum pools were analyzed on whole-proteome arrays. The 121 most reactive antigens identified during whole-proteome arrays were selected for further analysis with minimized microarrays that allowed for single sera analysis. From the 232 single sera; 145 (62.5\%) serum samples were reactive for at least one antigen. To discriminate between positive and negative serum samples; we created a panel of in total 18 antigens which identified $96 \%$ of all microarray positive samples. Antigens CT_858; CT_813 and CT_142 were most reactive. Comparison of antibody reactivity's among women with and without Ct related sequelae revealed that the reactivity of CT_813 and CT_142 was less common among women with PID compared to women without $(29.0 \%$ versus $58.6 \%, p=0.005$ and $25.8 \%$ versus $50.6 \%, p=0.017$ respectively). CT_858 was less common among CPP cases compared to controls (33.3\% versus $58.6 ; p=0.028)$. Using a whole-proteome array to select antigens for minimized arrays allows for the identification of novel informative antigens as general infection markers or disease associated antigens. 


\section{Introduction}

Chlamydia trachomatis (Ct) is the most frequently reported bacterial sexually transmitted infectious agent, and caused 127 million incident infections worldwide in 2016 [1]. Mostly women are affected, with an estimated worldwide prevalence of $4 \%$ among 15-49 years olds [1]. In women, Ct infections can ascend to the upper genital tract and cause pelvic inflammatory disease (PID), chronic pelvic pain (CPP), tubal factor infertility (TFI) and ectopic pregnancy (EP) [2].

$\mathrm{Ct}$ infections can be detected by either detecting the pathogen directly in clinical specimens, or by measuring antibodies against $\mathrm{Ct}$ in patient serum [3]. Nucleic acid amplification tests (NAAT) have replaced cell culture-based methods as the gold standard in clinical Ct diagnostics $[4,5]$. Although NAATs are highly suited to detect acute Ct infections, they are not able to detect past infections and may miss persistent, low load or upper genital tract infections. We hypothesize that when persistent upper genital tract infections are established and Ct resides in the form of aberrant reticulate bodies (RBs) inside epithelial cells, Ct as well as its DNA or RNA may no longer be detectable in swabs or other clinical specimens [6]. However, these infections may still be detectable indirectly through detection of Ct antibodies in serum [6].

The Microimmunofluorescence (MIF) test was formerly considered the gold standard for serodiagnosis of Ct infections [7]. In this test, serum antibodies against chlamydial elementary bodies (EB) are measured. Due to poor standardization, subjective microscopic readings and labor-intensive procedures of this method, alternative assay formats based on enzyme-linked immunosorbent assays (ELISA) have been developed [8]. However, most of these assays are limited to few defined chlamydial antigens. Commercially available assays use the Ct major outer membrane protein (MOMP), the heat shock protein 60 (hsp60), protease-like activity factor (CPAF) or translocated actin-recruiting phosphoprotein (TARP) as antigens [9-11]. The plasmid encoded Ct protein pGP3 is used in several published assays to detect Ct antibodies [12-15]. Another method to detect $\mathrm{Ct}$ antibodies to a large but still limited number of Ct proteins is multiplex serology, a bead-based high-throughput method used to detect antibodies to multiple antigens simultaneously [16]. This method has already been used to detect serum antibodies against selected Ct antigens in large cohorts [17,18].

Analysis of antibodies to the entire Ct proteome, consisting of 895 proteins, however allows the de novo identification of additional immunogenic Ct proteins. We recently developed a method to generate bacterial whole-proteome microarrays using a combination of Multiple Spotting Technique and cell-free, on-chip protein expression [19]. Bacterial proteins expressed on microarrays display antigenic epitopes, thereby providing an efficient method for immunoprofiling of patients and allowing de novo identification of antibodies associated with general infection as well as disease-related serum antibodies. Through comparison of antibody reactivity patterns, we identified antigens as markers for either general Ct infection or cervical cancer and validated these antigens using a high-throughput suspension bead array called multiplex serology [19] . De novo-identified disease-specific antibody profiles might be useful in clinical diagnosis, and allow the epidemiological investigation of the role of $\mathrm{Ct}$ infections in the development of different Ct-associated diseases. 
In the Netherlands Chlamydia Cohort Study (NECCST), women of reproductive age were followed over time to assess Ct disease progression [20]. Serum samples from a subsample of NECCST participants were analyzed with Ct whole-proteome microarrays for the following aims: to identify informative antigens to distinguish between Ct infected and non-infected participants, and to explore associations between novel Ct antigens and PID, CPP, ectopic pregnancy and TFI.

\section{Methods}

\section{Study Population}

Samples were selected from participants from NECCST. In NECCST, women of reproductive age are followed for a minimum of ten years until 2022 to assess Ct disease progression. NECCST participants previously participated in the Chlamydia Screening Implementation study (CSI) between 2008 and 2011 in which they participated in annual Ct NAAT tests [21]. In 2015-2016, these women were re-invited for NECCST. Participants filled in questionnaires concerning Ct infections, sexual risk behavior, PID, CPP, pregnancies and fertility. In case participants reported to be infertile they were asked how this diagnosis was made, the cause of infertility and possible treatments. Furthermore, additional informed consent was asked to request their medical files. Following the questionnaire, participants sent in a capillary blood sample to test for CT IgG to determine unnoticed infections using a Ct ELISA (CT IgG ELISA plus; Medac, Wedel, Germany).

\section{Sample Selection}

For the current study, we selected samples from a total of 5700 NECCST participants based on the following criteria: First, participants had given consent for the serum samples to be tested in other scientific research concerning sexually transmitted infections. Second, when sufficient amounts of serum volume, i.e., $>24 \mu \mathrm{L}$ were available. Lastly, when participants fell in any of the following three categories:

Group 1. Ct positive (i.e., tested positive by NAAT during the CSI, reported to have had a Ct infection, and/or tested positive for Ct IgG), but without any of the complications and at least once pregnant for a minimum of 20 weeks.

Group 2. Ct negative (i.e., no positive NAAT result during the CSI, no self-reported Ct infections and no Ct IgG antibodies), but with any of the complications, either PID, CPP, ectopic pregnancy or TFI. Group 3. Ct positive (i.e., tested positive by NAAT during the CSI, reported to have had a Ct infection, and/or tested positive for Ct $\operatorname{lgG}$ ) and with any of the complications, i.e., either PID, CPP, ectopic pregnancy or TFI.

In total, 259 serum samples were selected of which 143 (55.2\%) belonged to group 1, 80 (30.9\%) to group 2 and 36 (13.9\%) to group 3. Characteristics of the selected study participants are shown in Table 1. 
Table 1. Characteristics of the study population.

\begin{tabular}{|c|c|c|c|c|}
\hline & $\begin{array}{l}\text { Overall } \\
\mathrm{N}=259\end{array}$ & $\begin{array}{l}\text { Ct Positive Without } \\
\text { Complications } \\
\mathrm{N}=143 \text { (55.2) }\end{array}$ & $\begin{array}{l}\text { Ct Negative with } \\
\text { Complications } \\
\mathrm{N}=80(30.9)\end{array}$ & $\begin{array}{l}\text { Ct Positive with } \\
\text { Complications } \\
\mathrm{N}=36(13.9)\end{array}$ \\
\hline & $\mathrm{n}(\%)$ & $\mathrm{n}(\%)$ & $\mathrm{n}(\%)$ & $\mathrm{n}(\%)$ \\
\hline \multicolumn{5}{|l|}{ Age (years) } \\
\hline$<30$ & 77 (29.7) & $41(28.7)$ & 27 (33.8) & $9(25.0)$ \\
\hline $30-32$ & $69(26.6)$ & $31(21.7)$ & $22(27.5)$ & $16(44.4)$ \\
\hline $33-35$ & $75(29.0)$ & $45(31.5)$ & $23(28.8)$ & $7(19.4)$ \\
\hline$\geq 36$ & $38(14.7)$ & $26(18.2)$ & $8(10.0)$ & $4(11.1)$ \\
\hline \multicolumn{5}{|l|}{ CT history } \\
\hline Negative & $80(30.9)$ & $0(0.0)$ & $80(100.0)$ & $0(0.0)$ \\
\hline $\begin{array}{l}\text { Positive by at least } \\
\text { NAAT } \$\end{array}$ & $32(12.4)$ & $25(17.5)$ & $0(0.0)$ & $7(19.4)$ \\
\hline $\begin{array}{l}\text { Positive by at least } \\
\text { MOMP ELISA }\end{array}$ & $71(27.4)$ & $56(39.2)$ & $0(0.0)$ & $15(41.7)$ \\
\hline $\begin{array}{l}\text { Positive by only self- } \\
\text { reported infections }\end{array}$ & $76(29.3)$ & $62(43.4)$ & $0(0.0)$ & $14(38.9)$ \\
\hline \multicolumn{5}{|l|}{ Ct complications * } \\
\hline None & $143(56.9)$ & $143(100.0)$ & $0(0.0)$ & $0(0.0)$ \\
\hline PID & $52(20.1)$ & $0(0.0)$ & $31(38.8)$ & $21(58.3)$ \\
\hline CPP & $54(20.9)$ & $0(0.0)$ & $39(48.8)$ & $15(41.7)$ \\
\hline EP & $11(5.5)$ & $0(0.0)$ & $9(11.3)$ & $2(5.6)$ \\
\hline TFI & $13(5.0)$ & $0(0.0)$ & $8(10.0)$ & $5(13.9)$ \\
\hline
\end{tabular}

\$All women with a positive NAAT result were included. ${ }^{\circ}$ All women with a positive MOMP ELISA test, excluding women with a positive NAAT, were included. * Numbers do not add up to 100\%, i.e., women could have multiple sequelae. IQR = interquartile range. $\mathrm{PID}=$ pelvic inflammatory disease, $\mathrm{CPP}=$ chronic pelvic pain, EP = ectopic pregnancy, TFI = tubal factor infertility, MOMP = major outer membrane protein, ELISA = enzyme-linked immunosorbent assays.

\section{Whole-Proteome Microarray}

Whole-proteome microarrays representing the entire proteome of Ct serovar D with 895 proteins were generated as previously described by Hufnagel et al. 2018 [19]. In order to generate Ct whole-proteome arrays, two successive PCRs were performed for all open reading frames (ORFs) based on Ct genomic DNA. The products of the second PCR carrying all sequences necessary for transcription and translation (T7 Promoter, untranslated region, ribosome binding site, start codon, T7 Terminator), fusion peptide tags ( $\mathrm{N}$-terminal $6 \mathrm{x}$-His and $\mathrm{C}$-terminal V5 tags) were directly (without purification) used as the expression constructs for cell-free expression.

Protein microarrays expressing in situ the entire Ct proteome were generated using Multiple Spotting Technique [22]. During the first spotting step, the products of the second PCR were transferred onto Ni-NTA slides using a Nanoplotter 2 (GeSIM, Radeberg, Germany). Subsequently, the S30 T7 High-Yield Protein Expression Kit (Promega, Madison, WI, USA) was transferred directly on top of the expression construct spots. The slides were then incubated overnight in a humidified 
environment and proteins were expressed directly on the microarray slide and immobilized to the nickel surface.

Success of protein expression on the microarray was determined by incubation with fluorescenceconjugated antibodies directed against the $\mathrm{N}$ - and C-terminal fusion tags (Anti-6xHis, DyLight 650 (Abcam, Cambridge, UK) and anti-V5, Cy3 conjugate (Sigma-Aldrich, St. Louis, Missouri, USA)). The slides were scanned on a Power Scanner (Tecan, Männedorf, Switzerland) at $532 \mathrm{~nm}$ and $635 \mathrm{~nm}$ excitation wavelengths, respectively, and analyzed using the microarray acquisition and analysis software GenePix Pro 6.0 (Molecular Devices, Sunnyvale, CA, USA). Signal intensities were measured as median fluorescence intensity (MFI) signal of all pixels measured for one protein. In addition to the 895 CT proteins, we included the Epstein-Barr virus (EBV) viral capsid antigen p18 (VCA) as a positive control. An EBV seroprevalence of around 95\% in adults ensures a positive signal during proteome immunoassays (PIAs) even for Ct seronegative samples in the vast majority of cases. As a negative control (n.c.), both successive PCRs were performed without DNA template and the product of the second PCR was spotted as template for on-chip protein expression in one row of 20 replicates at the bottom of each slide. A protein was considered to be expressed if its signal intensity generated by the labeled antibodies to either the 6xHis or the V5 tag was higher than the mean plus five standard deviations of all n.c. replicates.

PIAs were performed by incubating serum samples in pools of five. These pools of five sera of $6 \mu \mathrm{l}$ each were generated based on long-term complications and if possible on different age categories of participants. In total, we had 53 pools either Ct positive with sequelae (separately for PID, CPP, ectopic pregnancy or TFI) or Ct negative with sequelae (Supplementary Figure S1). Because of the large sample, CT positive women without sequelae were pooled in different age categories i.e., $<30,30-32,33-35$, and $>35$ years. Of 53 pools, 48 were included in the PIA because of batch size. Five pools of controls were randomly excluded. Binding of a serum antibody to an antigen on the microarray was detected by a secondary fluorescence-conjugated antibody (Alexa Fluor 647-conjugated goat anti-human IgA, IgG, IgM; Jackson Immuno Research, West grove, PA, USA). The microarrays were scanned at an excitation wavelength of $635 \mathrm{~nm}$. The signal intensity obtained for a given antigen was considered proportional to the amount of primary antibody bound on the microarray.

Quality (QC) control of PIAs was performed by assessing the scanned images of each slide, and representing each PIA as a plot in which the acquired MFI data is visualized according to its position on the slide.

This allows for the recognition of technical artefacts on the individual slides, such as gradient effects of the background or MFI signals. Whole-proteome slides were excluded if they met one of the following criteria:

(i) gradient effects (e.g., loss of reactivity from top to bottom of a slide);

(ii) smearing of spots (due to e.g., insufficient washing);

(iii) disproportional high readouts for all negative controls (leading to the detection of false negative readouts of antigens).

As QC procedures were qualitative in nature, they were performed independently by two scientists $(\mathrm{KH}$ and $\mathrm{CH})$. 


\section{Selection of Informative Antigens}

The files generated with GenePix Pro 6.0 contained the MFIs values for each processed slide and were analyzed using the statistical programming language $R$ (R Core Team, 2018).

For analysis of the PIAs, data were imported to $\mathrm{R}$ and seropositivity was determined by neighborhood averaging, i.e., by calculating a specific threshold for each spot position in order to address local variation of the signal intensity across the array (Equation (1)). The threshold criterion takes the relative distances of the spots into account, so that for the calculation of one spot's threshold the MFI values of the 50 closest spots are considered, not distinguishing between negative controls and protein spots but excluding positive controls.

$$
\begin{gathered}
\text { Seropositive }=M F I_{\text {spot }}>\left(M E D\left(M F I_{50 \text { closest spots }}\right)+3 M A D\left(M F I_{50 \text { closest spots }}\right)\right) \\
M E D=\text { median, } M A D=\text { median absolute deviation }
\end{gathered}
$$

Different approaches to calculate a threshold were evaluated in order to determine the most robust approach. The calculation and comparison of three different threshold criteria are described in Supplementary Methods S1. After removing slides from the dataset based on the described quality control criteria, Ct proteins were selected for further spotting of microarrays that allow for the incubation of 8 single sera per slide. This new spotting layout limits the number of spots per block to 140 (121 Ct proteins, 4 positive and 15 negative controls). The 120 most reactive antigens identified during incubation of serum pools on whole-proteome microarrays were included in the minimized microarray. In addition, CT_798 was included since it was identified during a previous study using Ct whole-proteome microarrays and was already validated using multiplex serology [19]. This resulted in a total number of 121 antigens. Although antibodies that exceeded the threshold-fold only once might be of low prevalence or present only in low titers, they might nevertheless be promising markers for general infection or disease association. The 121 antigens were expressed on microarrays containing eight blocks separated by frames. Immunoassays as well as quality controls were performed as described for whole-proteome microarrays. Since the 15 negative controls were distributed over the whole slide a weighted negative control approach was used. For each spot, an individual threshold is calculated based on distance-weighted negative control signals. The threshold $t$ is defined for each spot $j$ as (Equation (2)):

$$
t_{j}=\bar{z}_{j}+3 \cdot \sqrt{\hat{\sigma}_{j}^{2}}
$$

With $\overline{z_{j}}$ being the weighted mean of the MFI values of the negative controls $z_{i}$ and $\hat{\sigma}_{j}^{2}$ being the respective weighted variance. The weights $w_{i, j}$ are defined as the reciprocal euclidean distances of the negative controls $z_{i}$ to the spots $j$ with $\mathrm{x}$ and $\mathrm{y}$ being the respective cartesian coordinates of the negative controls $i$ and spots $j$ on the array (Equations (3-5)):

$$
\begin{gathered}
w_{i, j}={\sqrt{\left(x_{j}-x_{i}\right)^{2}+\left(y_{j}-y_{i}\right)^{2}}}^{-1} \\
\bar{z}_{j}=\frac{\sum_{i=1}^{n} w_{i, j} z_{i}}{\sum_{i=1}^{n} w_{i, j}}
\end{gathered}
$$




$$
\hat{\sigma}_{j}^{2}=\frac{\sum_{i=1}^{n}\left(z_{i}-\bar{z}_{j}\right)^{2}}{\sum_{i=1}^{n} w_{i, j}}
$$

\section{Statistical Analyses}

Descriptive analyses were performed and characteristics of the study population were presented.

1. Reactivity of the antigens.

Percentage positive was described for serum samples positive for at least one antigen up until at least five positive antigens in the minimized array. Furthermore, a panel of the most distinctive antigens to capture all the serum samples positive for at least one antigen were determined. Last, the fifty most reactive antigens were identified based on the number of positive sera.

2. Exploration of $\mathrm{Ct}$ antigens related to sequelae.

The percentage positive, the median number of positive antigens and the most frequent found antigens (three highest percentages of at least $40 \%$ ) were described for Ct positive without complications (group 1), Ct negative with complications (group 2), and Ct positive with complications (group 3). Proportions of positive serum samples against specific antigens were compared between PID-, CPP-, EP- and TFI-cases and controls (Ct positive without complications) using chi-squared tests.

Statistical analyses were performed using R studio and STATA (Version 14.2; StataCorp, College Station, TX, USA).

This study was approved by the Medical Ethical VU Medical Center, Amsterdam, the Netherlands (NL 51553.094.14/2015.903(A2019.336)). Date of approval Committee 10/13/2015. All participants provided informed consent.

\section{Results}

\section{Antigen Selection}

We were able to successfully express more than $96 \%$ of all $895 \mathrm{Ct}$ proteins. The result of the expression staining using labelled antibodies against the terminal fusion tags of the $895 \mathrm{Ct}$ proteins is illustrated in 2a. In total, PIAs were performed for 48 serum pools of the NECCST study. Exemplary results for three PIAs are illustrated in Figure 1b-d. Of these 48 slides, six were removed after quality control. Two slides were removed due to vertical gradients based on incomplete protein expression, and two other slides showed horizontal gradients probably due to insufficient washing; two additional slides did not pass quality control because of extensive smearing between spots. Seventeen of the analyzed pools only comprised sera from women who were considered Ct negative based on previous tests or questionnaire. The serum samples were nevertheless included, since they were all collected from women with $\mathrm{Ct}$ associated complications. Only five of these pools were identified as truly negative on the Ct whole-proteome microarray. Based on the results of the 42 remaining whole-proteome arrays (Figure 2), we selected the 121 
most reactive and thus potentially informative antigens. No differences were seen between the different pools. The 121 most reactive antigens represent $13.5 \%$ of the complete Ct proteome. Fourteen of the 121 antigens showed $\mathrm{N}$-terminal but no $\mathrm{C}$-terminal expression signals on wholeproteome arrays. Although no C-terminal expression signal was detected, these 14 antigens were nevertheless recognized by serum antibodies of Ct-infected individuals at least once. A list of the 120 most reactive $\mathrm{Ct}$ antigens and their corresponding threshold-fold values can be found in Supplementary Table S1. CT_798 is not included in the table since it was not identified as a reactive antigen in this study but was added due to previous findings.

a

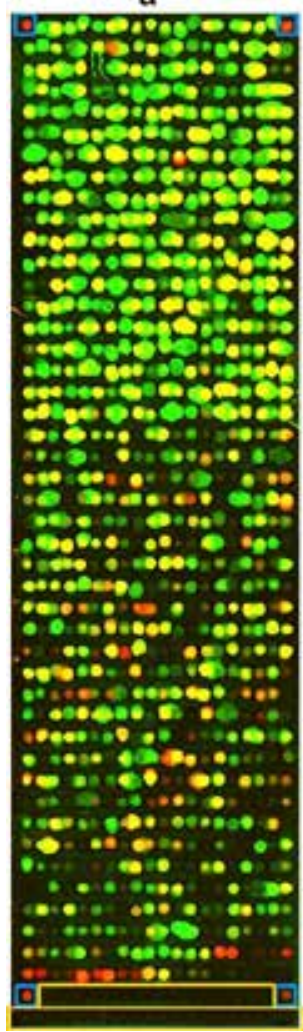

b

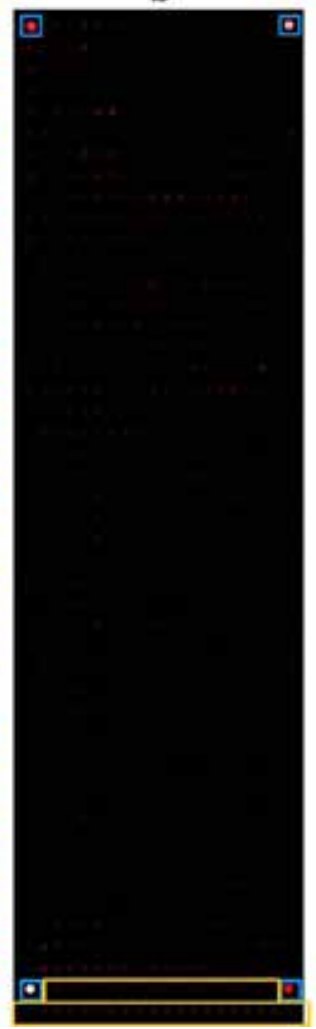

c

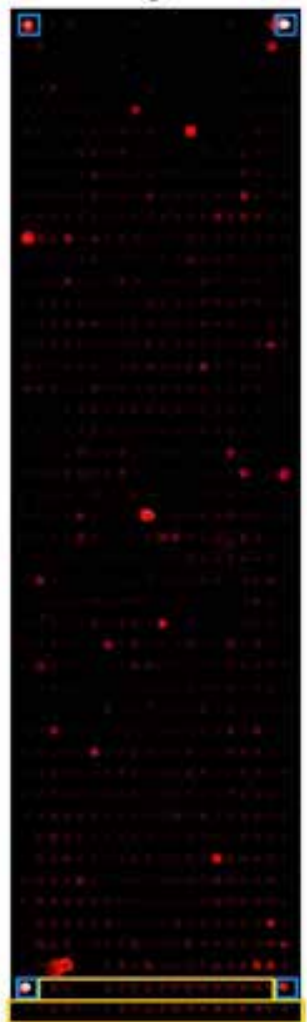

d

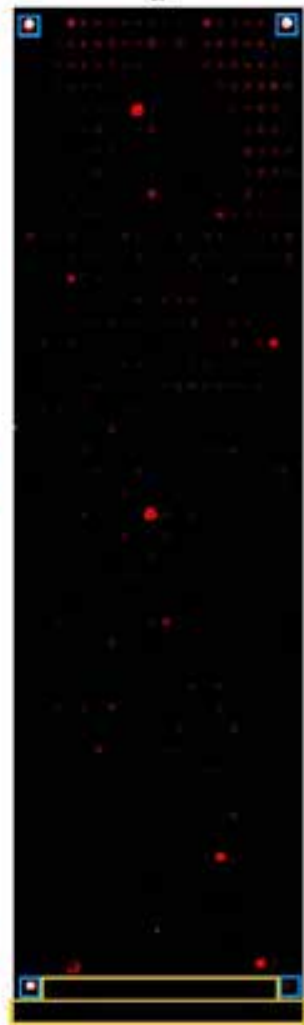

Figure 1. Proteome immunoassays (PIA) using pools of five sera. In total, $895 \mathrm{Ct}$ proteins were spotted on one array. Negative and positive controls are highlighted with yellow and blue boxes, respectively. (a): Expression control using fluorescence-conjugated antibodies against the terminal tags of the Ct proteins (green signal: anti-V5 antibody; red signal: anti-His antibody; yellow signal: overlay of both signals). (b): No signal was obtained with a pool of five sera from Ct-uninfected women for any of the Ct antigens but showed reactivities with all four positive controls. (c,d): Results of two PIAs with pools of five sera from women infected with Ct. 
a

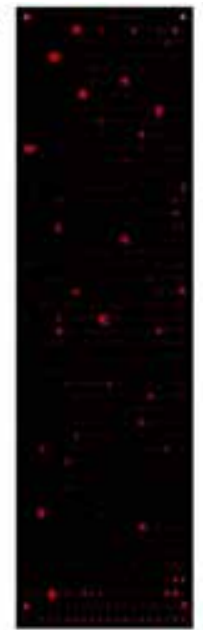

b

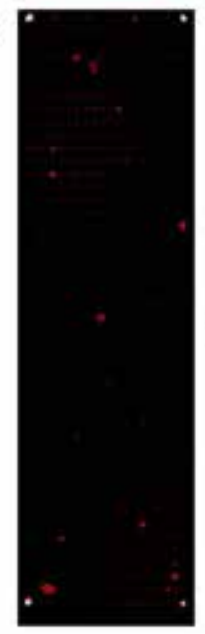

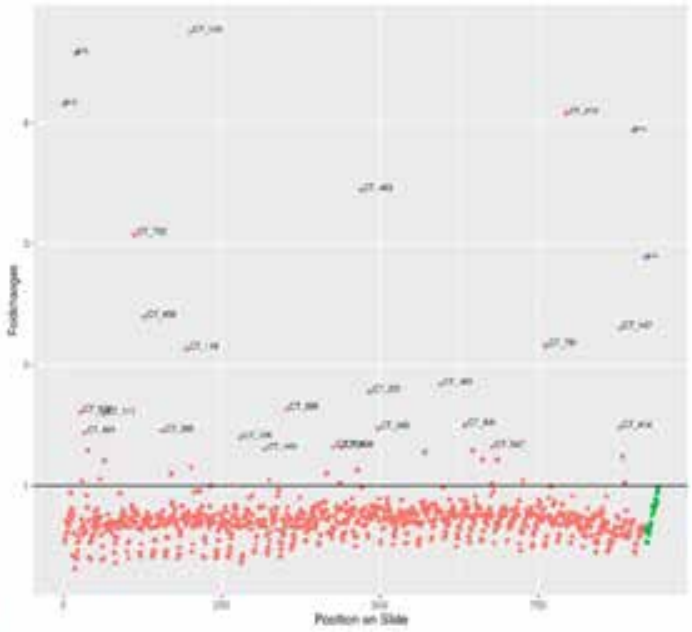

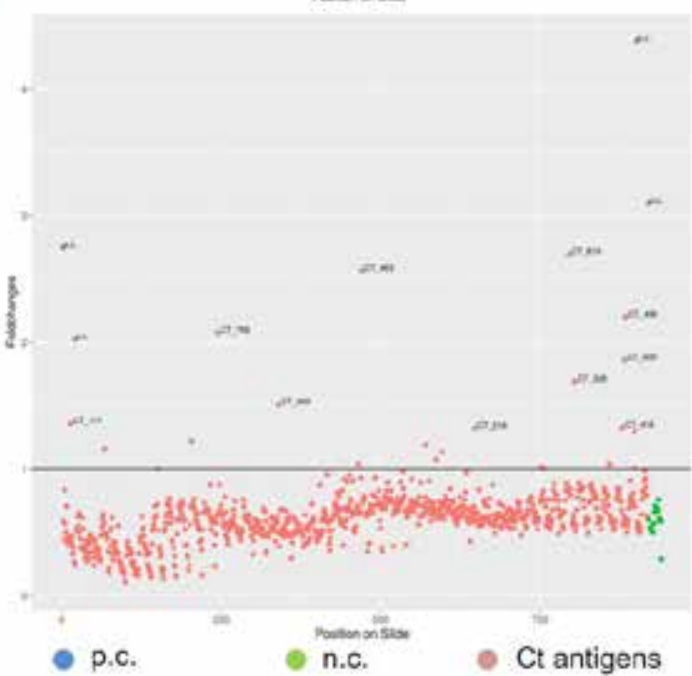

Figure 2. Result of two PIAs using pools of five sera from Ct-infected patients with (a) EP and (b) PID. Pictures on the left show scanned images of microarray slides after performing PIAs. Red and white spots indicate reactivities of the incubated serum pool with the antigen expressed on this position of the slide. The graphs on the right side illustrate signal intensity by Foldchanges of each antigen. Antigens are plotted onto the $x$-axis based on their position on the microarray slide. The threshold is illustrated by a straight black line in the graph. Individual Ct proteins with elevated reactivity are labeled with individual Ct ORF numbers. The Epstein-Barr virus viral capsid antigen was spotted as a positive control (p.c., blue) in all four corners. Negative controls (n.c., green) were spotted in the last row of each slide. An antigen was considered to be immunogenic if its signal was higher than the defined threshold (indicated by the black line $y=1$ ).

\section{Single Sera Outcomes}

Of 259 selected serum samples, 248 single samples were tested and following quality control, 16 were excluded, (Figure 3). No significant differences in characteristics were found between the 
initial 259 samples and the in total 232 single included sera. Typical results and corresponding data analysis are shown in Figure 4.

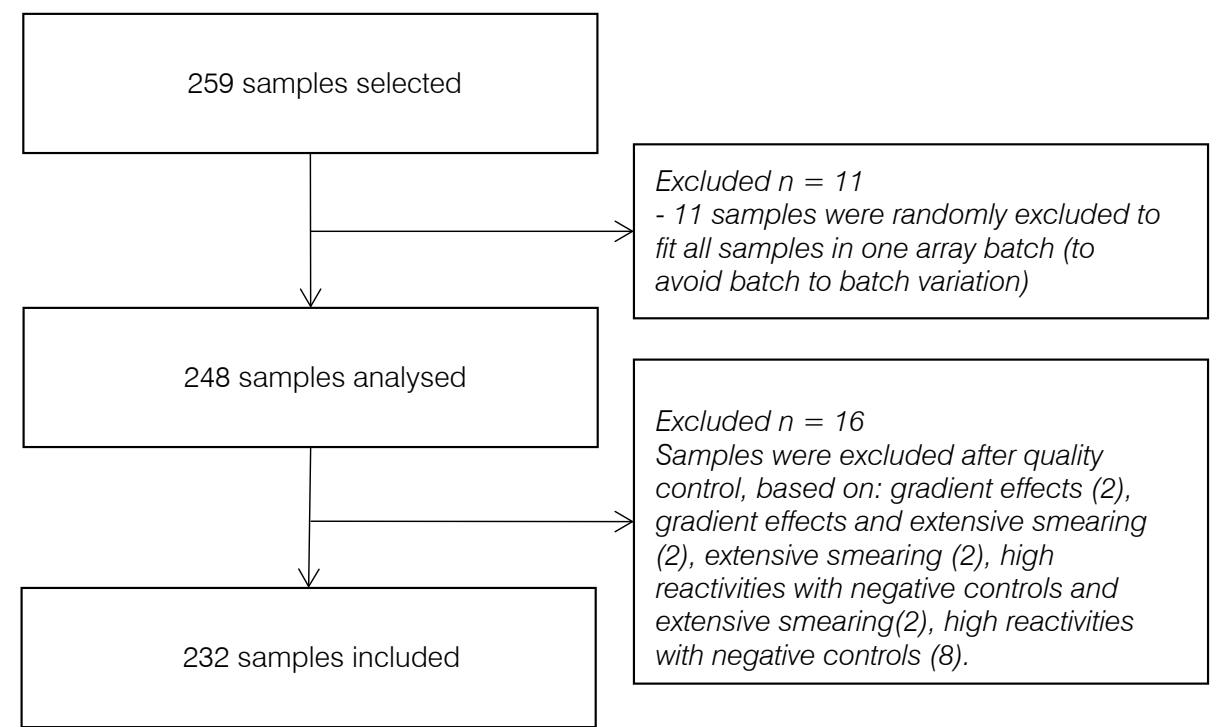

Figure 3. Flowchart of the included samples.

\section{Reactivity of the antigens}

From the 232 single sera, 145 (62.5\%) serum samples were reactive for at least one antigen and 76 (32.8\%) serum samples for at least five antigens (Figure 5). The median number of reactive antigens was five (IQR 2-11) (Table 2). The percentage of reactive sera was highest among group 3: Ct positive women with complications group 3 (78.1\%), while among group 1 (Ct positive controls) $65.9 \%$ of all tested sera showed reactivity with at least one antigen. Lowest reactivities were observed among group 2 (Ct negative women with complications) which was $48.5 \%, p=$ 0.008. Using a stricter definition of seropositive (i.e., at least positive against two, three or five antigens) showed similar patterns though with lower percentage of reactive sera and no significant difference between the three groups. Among serum samples from women that were previously tested as Ct-NAAT positive, $81.3 \%$ showed reactivity with antigens on the microarray. This was 78.2\% among MOMP ELISA positives and 55.7\% among self-reported positives only, $p=0.001$. Of previously Ct-NAAT negatives, $51.5 \%$ were negative in the minimized array. 
a
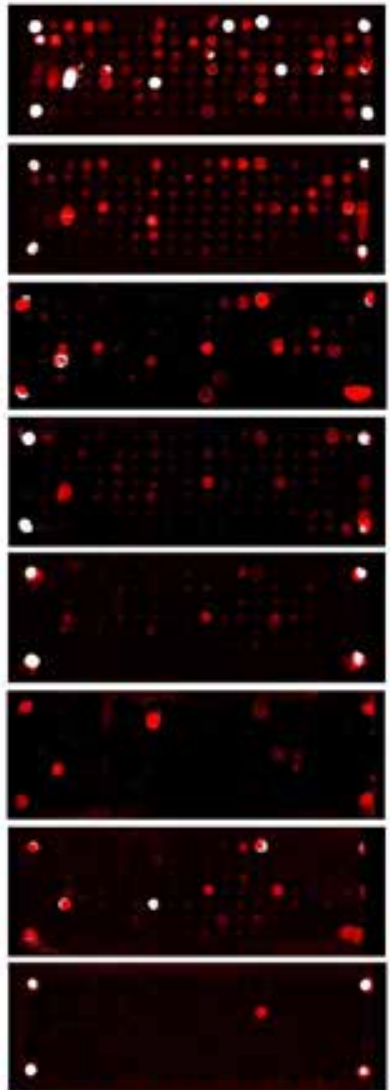

-

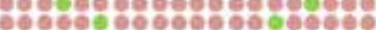

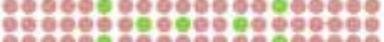

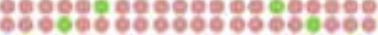

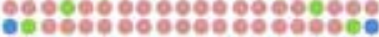

b
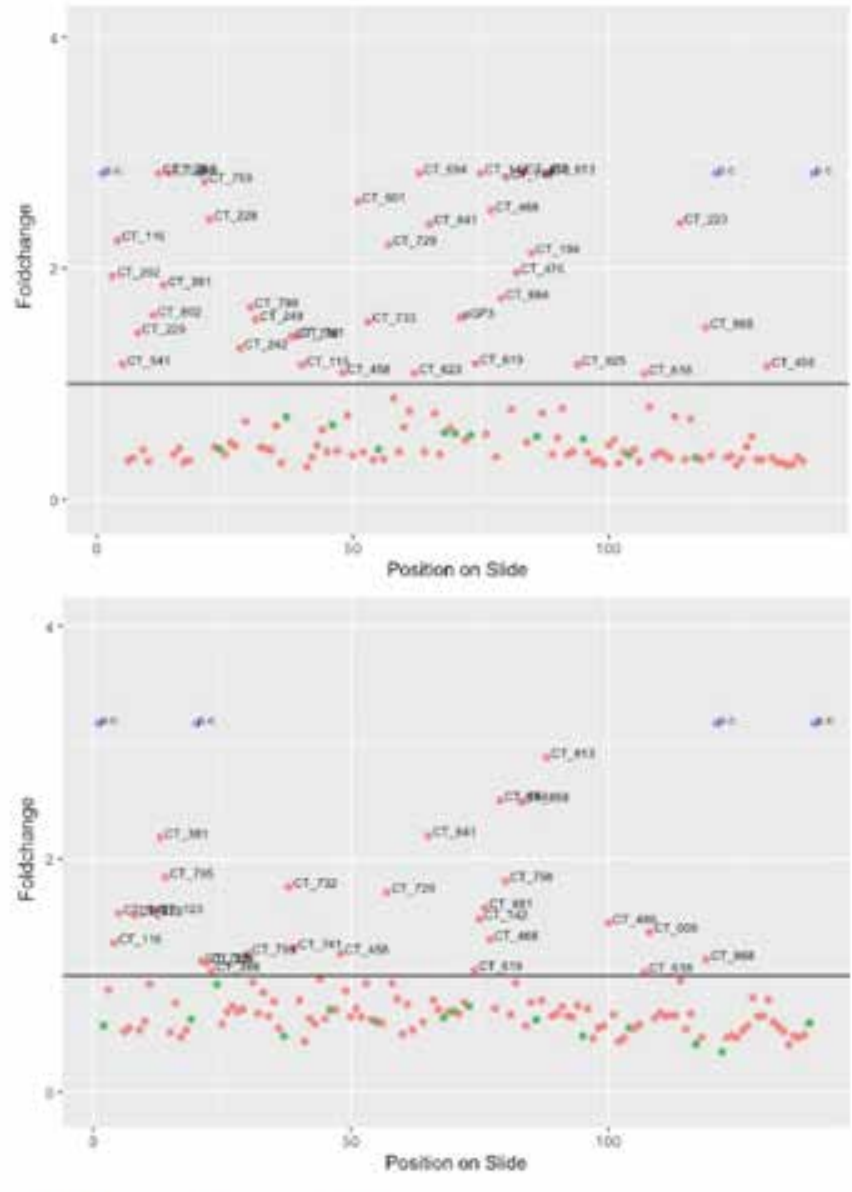

- p.c. $\mathrm{Cl}$. $\mathrm{Ct}$ antigens

Figure 4. Results obtained after performing immunoassays with single serum samples on minimized arrays containing 121 identified $\mathrm{Ct}$ antigens. a: scanned images of immunoassays with 8 single sera from Ctinfected women and layout of each of the eight blocks. Red and white spots indicate reactivities of the incubated serum sample with the antigen expressed on this position of the slide. $\mathbf{b}$ : data analysis for the two topmost blocks in panel a. The graphs illustrate signal intensity by Foldchanges of each antigen. Antigens are plotted onto the $\mathrm{x}$-axis based on their position on the microarray slide. The threshold is illustrated by a straight black line in the graph. Individual $\mathrm{Ct}$ proteins with elevated reactivity are labeled with individual $\mathrm{Ct}$ ORF numbers. The Epstein-Barr Virus Viral Capsid Antigen was spotted as a positive control (p.c., blue) in all four corners. Negative controls (n.c., green) were spotted throughout each block. An antigen was considered to be immunogenic if its signal was higher than the defined threshold (indicated by the black line $y=1)$. 


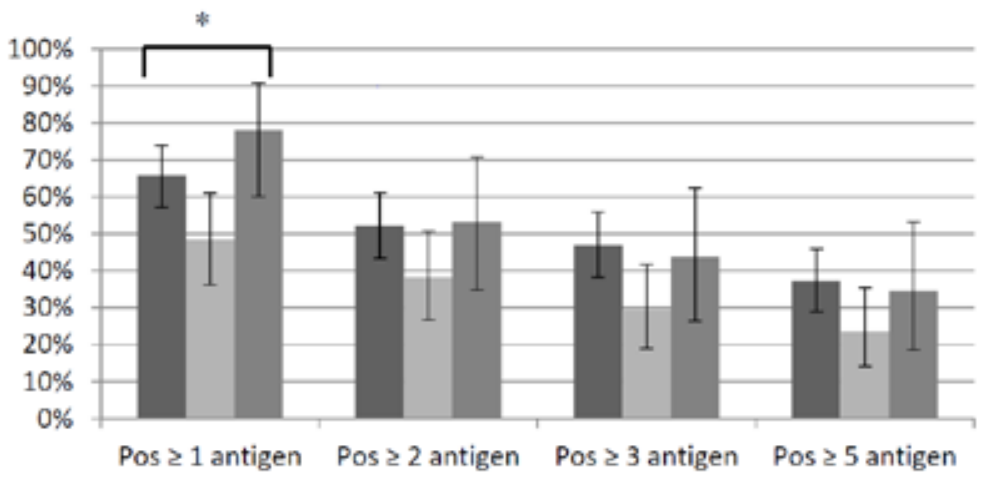

1. Ct pos without complications $=2$. Ct neg with complications $\quad$ a. Ct pos with complications

Figure 5. Percentage positive by minimized array between Ct positive without complications, Ct negative with complications and Ct positive with complications. * $=$ a significant difference $(p<0.05)$ in percentage positive.

Table 2. Percentage positive serum samples by different subgroups.

\begin{tabular}{|c|c|c|c|}
\hline & $\begin{array}{l}\text { Positive Serum Samples } \\
N(\%, 95 \% \mathrm{Cl})\end{array}$ & $\begin{array}{l}\text { No. of Positive } \\
\text { Antigens } \\
\text { Median (IQR) }\end{array}$ & $\begin{array}{l}\text { Top Three Antigens with Highest } \\
\text { Seroprevalence ( } \geq 40 \%) \\
\%\end{array}$ \\
\hline $\begin{array}{l}\text { Controls-Ct } \\
\text { positive }\end{array}$ & $87(65.9,57.2-73.9)$ & $5(2-15)$ & $\begin{array}{l}\text { CT_858 (58.6), CT_813 (58.6), } \\
\text { CT_142 (50.6), }\end{array}$ \\
\hline Cases-Ct negative & $33(48.5,36.2-61.0)$ & $4(2-7)$ & - \\
\hline PID positive & $28(57.1,37.2-75.5)$ & $3.5(2-6)$ & - \\
\hline CPP positive & $13(43.3,25.5-62.6)$ & $5(1-6)$ & - \\
\hline EP positive & $2(22.2,2.8-60.0)$ & $10.5(7-14)$ & NA \\
\hline TFI positive & $4(66.7,22.3-95.7)$ & $7(2-14)$ & $\begin{array}{l}\text { CT_123 (50.0), CT_142 (50.0), } \\
\text { CT_664 (50.0), CT_858 (50.0), } \\
\text { CT_104 (50.0), CT_813 (50.0) }\end{array}$ \\
\hline Cases-Ct positive & $25(78.1,60.0-90.7)$ & $3(1-9)$ & $\begin{array}{l}\text { CT_142 (56.0), CT_858 (52.0), } \\
\text { CT_813 (44.0) }\end{array}$ \\
\hline PID positive & $15(83.3,58.6-96.4)$ & $2(1-9)$ & $\begin{array}{l}\text { CT_}^{-} 858(53.3), C_{-} 142(40.0), \\
\left.\text { CT_813 }^{-} 40.0\right),\end{array}$ \\
\hline CPP positive & $11(78.6,49.2-95.3)$ & $6(1-11)$ & $\begin{array}{l}\text { CT }^{-} 142(54.5), C_{1} 841 \text { (45.5), } \\
\text { CT }^{-} 858(45.5), \text { CT }^{-} 813(45.5)\end{array}$ \\
\hline EP positive & $1(50.0,1.25-98.7)$ & 1 & NA \\
\hline TFI positive & $5(100.0)$ & $6(2-9)$ & CT_142 (100.0)*, CT_858 (60.0) * \\
\hline
\end{tabular}

Controls-Ct positive $=$ tested positive for Ct by NAAT, self-reported positive test or tested positive in Medac Momp assay but without any complication. Cases-Ct negatives = never tested positive for Ct by NAAT, no self-reported infections and negative for Ct antibodies by Medac Momp assay but with any of the complications (i.e., PID, CPP, EP or TFI). Ct positives = tested positive for Ct by NAAT, self-reported positive test or tested positive in Medac Momp assay but with any of the complications. PID = pelvic inflammatory disease, $\mathrm{CPP}=$ chronic pelvic pain, $\mathrm{EP}=$ ectopic pregnancy, and TFI = tubal factor infertility. IQR $=$ interquartile range. * = only the first two highest percentages were described. NA = not applicable (sample size too small). 
To discriminate between serum samples positive for at least one antigen and serum samples negative for all antigens, we created a panel of in total 18 antigens which can identify $96 \%$ of all serum samples positive for at least one antigen, Figure 6.

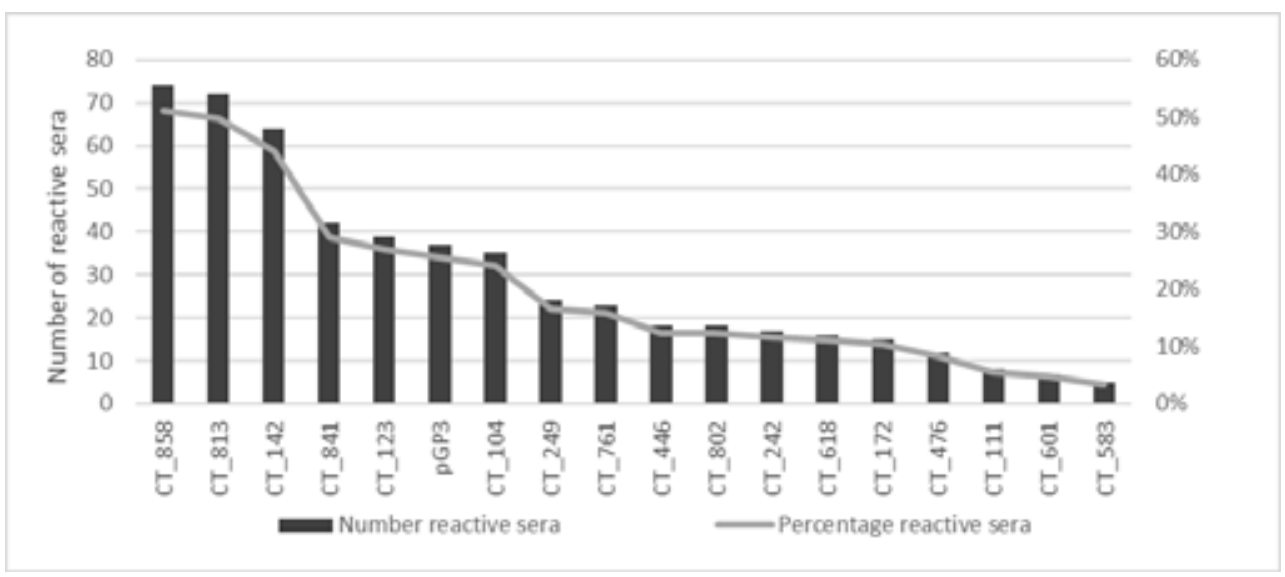

Figure 6. Panel of 18 antigens to identify $96 \%$ of all positive serum samples in the cohort.

The fifty most reactive antigens are shown in Table 3. CT_858 (CPAF) and CT_813 were most reactive and positive in $51 \%$ and $49 \%$ among serum samples positive for at least 1 antigen, respectively. Antigen CT_142 was reactive in 44\% of the positive samples. Besides CPAF other known immunogenic antigens such as pGP3, CT_681 (MOMP), CT_456 (TARP), and CT_110 (Hsp60) were also among the fifty most reactive antigens. 


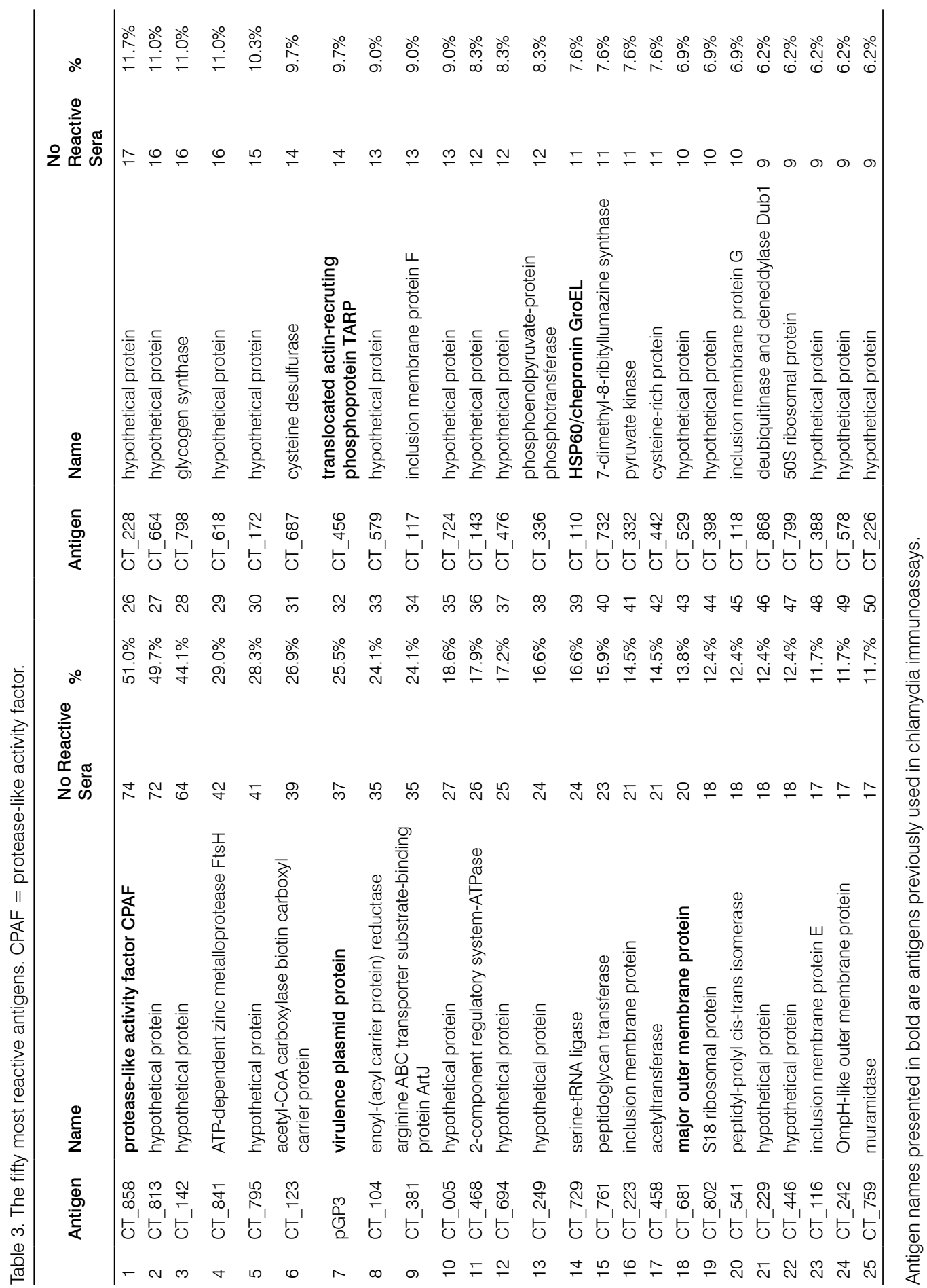




\section{Exploration of $\mathrm{Ct}$ antigens related to sequelae}

Of eleven TFI cases, nine (81.2\%) were positive for antibodies against at least one antigen, most frequently CT_142 and CT_858 (Table 2). Thirty out of forty-six PID cases were positive against at least one antigen (67.4\%), which was most often CT_858. Among CPP and EP cases, 54.5\% and only $27.3 \%$ were positive for at least one antigen, respectively.

Table 4 shows the presence of antibodies against antigens that were significantly different between cases (i.e., PID or CPP positive) and controls (Ct positive without any complication). The presence of antibodies against CT_813 and CT_142 was less common among women with PID compared to women without PID. In CPP positive women, the presence of antibodies against CT_858 was less common compared to women without CPP. No significant differences in the presence of specific antibodies were found between TFI or EP cases compared to controls.

Table 4. The presence of specific antigens in controls and cases with pelvic inflammatory disease and chronic pelvic pain

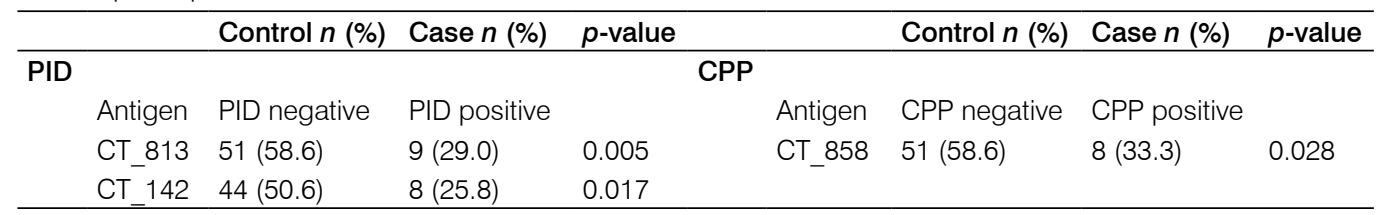

$\mathrm{PID}=$ pelvic inflammatory disease, $\mathrm{CPP}=$ chronic pelvic pain. Control $=$ Ct positive without any complication . Case $=$ is either Ct positive of negative (in NECCST) but with PID or CPP.

\section{Discussion}

Using Ct whole-proteome microarrays and minimized arrays, we aimed to identify informative antigens to distinguish between $\mathrm{Ct}$ infected and non-infected women and to identify antigens associated with Ct-associated disease outcomes. To this end, we analyzed 232 samples from the NECCST study, a cohort study which follows women of reproductive age to assess Ct disease progression. We found 145 (63\%) serum samples to be positive for at least one antigen in the minimized array. The antigens CT_858, CT_813 and CT_142 were most reactive in our sample of women. Among PID positive women, fewer serum samples were positive for the antigens CT_142 and CT_813 compared to women without PID. In CPP positive women, CT_858 was less common compared to controls.

In the Ct whole-proteome arrays, 860 out of all $895 \mathrm{Ct}$ proteins were successfully expressed. Of all expressed Ct proteins, 121 antigens were selected for the minimized array based on analysis of pools of sera. Using minimized arrays that included the 121 selected antigens, 232 single serum samples were tested. Overall, 63\% of sera were positive for at least one of these 121 antigens. In sera from women who previously tested positive for Ct by NAAT, $81 \%$ were positive for at least one antigen. Considering that women were Ct NAAT positive between 2008 and 2011 , that antibodies can wane over time, and given not every infected woman seroconverts [12,23], this is a high agreement. This agreement is higher than other published serological assays which 
utilize only one or few Ct antigens for serodiagnosis of Ct infections. In a study in which five Ct antibody assays with different target antigens were compared, seropositivity was only between $38 \%$ and $68 \%$ after a median of three years and a minimum of six months after infection [24]. On the other hand agreement with Ct NAAT negatives was only $51.5 \%$, because $48.5 \%$ of previously Ct-NAAT negatives were reactive for at least one antigen in the minimized array. This is difficult to interpret. Although NAAT tests are very sensitive and specific, the test can only measure current infections and does not rule out past infections [3]. In addition, samples from these women were tested with the Medac MOMP ELISA, but this assay only detects about $40 \%$ of past infections [25-27]. Furthermore, these Ct-NAAT negative tested women, were all women with CPP, PID, ectopic pregnancy or TFI, complications which could relate to Ct infections and thereby possible exposed to past Ct infection. Nevertheless, it is unlikely that all these $48.5 \%$ microarray positives are true positives. For further research this array has to be tested in a population with true or reliable Ct negatives, for example in blood samples from infants or children or nuns.

In our cohort, CT_858 and CT_813 were identified as the most reactive antigens and showed reactivities with $51 \%$ and $49 \%$ of all Ct seropositive sera, respectively. While CT_858 alone would react only with $51 \%$ of all Ct seropositive samples, a combination of CT_813 and CT_858 would result in a 65\% detection rate. Furthermore, a panel of CT_858, CT_813, CT_142 and CT_104 would be able to identify $78 \%$ of all seropositive samples. This means, we observe a higher coverage with more antigens and the coverage rate cumulatively increases with number of antigens due to different reactivity patterns of individual samples. Therefore, we suggest a panel of 18 antigens to identify $96 \%$ of all Ct seropositive sera. Each of the six serum samples not reacting with any of the 18 antigens shown in Figure 6 showed only extremely weak reactivities with a single additional antigen. These reactivities might be unspecific signals. However, it cannot be excluded that these sera are true Ct seropositive samples and we miss them with our panel of 18 antigens. An ideal platform for such a serological assay would be high-throughput bead-based multiplex serology. For the bacterium Helicobacter pylori a panel of 17 antigens is already widely used to detect differential antibody responses [28].

CT_858 is a serine protease known as chlamydial proteasome/protease-like activity factor (CPAF) which is secreted from the chlamydial intracellular inclusion into the host cell cytoplasm [29]. The role of CPAF as a virulence factor that degrades host proteins is currently widely discussed $[30,31]$. As a secreted virulence factor that is expressed during Ct infection, CPAF is likely a target for host antibodies and was therefore already used in published serological assays [32,33]. The hypothetical protein CT_813 was identified as an antigen to determine general Ct infections and was described by Chen et al. to be located within the Ct inclusion membrane. They were able to detect the protein $12 \mathrm{~h}$ after chlamydial infection. In addition, it was shown that CT_813 is present in the inclusion membrane during the entire growth cycle and an interaction of this protein with host cells was described [34]. CT_813 is expressed in many Ct serovars but not in other chlamydial species, which makes it a promising candidate to specifically detect Ct infections without any cross-reactivity to other species. Another promising antigen identified during this study to detect general Ct infections is the hypothetical protein CT_142. So far, and to the best of our knowledge, nothing is published about its function and role in the Ct infection cycle. Previously, CT_813 and 
CT_142 were validated as general infection markers in a large seroepidemiological study using multiplex serology [19]. Among women positive for at least one antigen in our study, antibodies against antigens CT_813 and CT_142 were less common among PID cases compared to women without PID. This might indicate an effective immune response that may render a protective effect against PID. Among CPP cases, CT 858 was less common compared to women without CPP. More research is needed to gain insight in the functions and roles of CT_813, CT_142 and CT_858 in Ct-associated disease. Disease specific antigens for EP or TFI were not found.

Besides the identification of novel immunogenic antigens we were able to confirm known immunogenic antigens as infection markers, like the major outer membrane protein (CT_681), the plasmid encoded protein pGP3, and Hsp60 (CT_110) all of which are already used in both commercial and non-commercial serological assays to detect Ct serum antibodies $[9,12]$ The most reactive antigens (CT_858, CT_813, CT_142, CT_841, CT_795, CT_123, pGP3) which reacted with more than $25 \%$ of all tested sera, were also identified as highly immunogenic antigens when analyzing serum samples from the Mongolian population based cross-sectional HPV prevalence study on Ct whole-proteome microarrays [19]. The identification of the same set of most reactive antigens in two independent studies indicates that these antigens are promising markers to detect general Ct infections.

In total $63 \%$ of all tested sera reacted at least with one $\mathrm{Ct}$ antigen on the microarrays. The humoral immune system of these $\mathrm{Ct}$ infected women recognizes and responds to different $\mathrm{Ct}$ antigens by eliciting a variety of antibodies. The remaining $38 \%(n=87)$ of the tested serum samples did not result in significant signals, although $52(60 \%)$ of these were from women previously tested positive for Ct (either self-report or study NAAT) or ELISA assay. The immune system of these individuals did not produce antibodies against Ct antigens included in our assay. However, these women showed a specific reactivity with the positive control (EBV VCA) as illustrated in Figure 4. We observed an EBV seroprevalence of $>90 \%$ in minimized arrays both for Ct-infected as well as Ct-uninfected sera, which corresponds well to the expected seroprevalence in adults of around 95\%.

\section{Limitations}

The major outer membrane protein MOMP (CT_681) reacted only with 20 analyzed serum samples in this study. Since this antigen is used in many commercially available assays, we expected to detect reactivities to this particular antigen more often. However, these assays often utilize linear peptides that might, in this case, be a better target for serum antibodies. If the full length MOMP on the microarray is incorrectly folded, the epitope might not be accessible to the serum antibodies. Incorrect protein folding or aggregation might result in false negative signals and therefore some immunogenic antigens might not be detectable on the microarray. However, since we were able to detect high signals for antigens such as CT_858, CT_813 and CT_142 this may be a limitation for some but not for all antigens.

Second, we had a small sample size to identify disease specific antigens, especially for EP and TFI which consisted of only three and nine seropositive serum samples, respectively. This limitation makes it impossible to come to definitive conclusions about associations between 
specific antigens and these Ct outcomes.

The third limitation is the possibility of antibody cross-reactivity. In order to investigate crossreactivities of the identified antigens with antibodies to antigens of the closely related organism Chlamydophila pneumoniae (Cp), homologies to $\mathrm{Cp}$ proteins were analyzed using the NCBI protein blast tool (https://blast.ncbi.nlm.nih.gov/Blast.cgi?PAGE=Proteins). The hypothetical protein CT_142 showed an identity of 35\% to a hypothetical protein of Cp while CT_858 showed $48 \%$ sequence identity to CPAF of $\mathrm{Cp}$. Therefore, cross-reactivities cannot be excluded for these antigens, although substantial cross-reactivity usually requires a higher degree of amino acid relatedness. However, CT_813 showed no homologies to any protein of Cp. Therefore, cross-reactivity for CT_813 with antibodies against Cp antigens is unlikely. Nevertheless, these proteins should be validated in a cohort with serum samples that are truly seronegative for $\mathrm{Ct}$ and positive for any of the other chlamydial species to test possible cross-reactivity. In addition, highly immunogenic proteins of closely related species, like the major outer membrane protein of $\mathrm{Cp}$, should be included in whole-proteome and minimized arrays to test for possible cross-reactivities.

\section{Conclusion}

Using a whole-proteome array to select antigens for minimized arrays allows for the identification of novel and potentially promising antigens to distinguish between $\mathrm{Ct}$ negative and Ct positive women. In our minimized Ct array containing 121 antigens, seropositivity was $81 \%$ in women previously tested Ct NAAT positive. A panel of 18 antigens of these 121 antigens can identify 96\% of all microarray seropositive samples in this cohort. Antigens CT_858, CT_813 and CT_142 were most reactive and might be of value for future $\mathrm{Ct}$ antibody assays. 


\section{References}

1. Rowley, J.; Vander Hoorn, S.; Korenromp, E.; Low, N.; Unemo, M.; Abu-Raddad, L.J.; Chico, R.M.; Smolak, A.; Newman, L.; Gottlieb, S.; 9. et al. Chlamydia, gonorrhoea, trichomoniasis and syphilis: Global prevalence and incidence estimates, 2016. Bull. World Health Organ. 2019, 97, 548-562, doi:10.2471/BLT.18.228486.

2. Haggerty, C.L.; Gottlieb, S.L.; Taylor, B.D.; Low, N.; Xu, F.; Ness, R.B. Risk of sequelae after Chlamydia trachomatis genital infection in women. J. Infect. Dis 2010, 201, S134-S155, doi:10.1086/652395.

3. Meyer, T. Diagnostic procedures to detect Chlamydia trachomatis infections. Microorganisms 2016, 4, doi:10.3390/ microorganisms 4030025 .

4. Centers for Disease Control and Prevention. Recommendations for the laboratorybased detection of Chlamydia trachomatis and Neisseria gonorrhoeae-2014. MMWR Recomm. Rep. 2014, 63, 1-19.

5. Nwokolo, N.C.; Dragovic, B.; Patel, S.; Tong, C.Y.; Barker, G.; Radcliffe, K. 2015 UK national guideline for the management of infection with Chlamydia trachomatis. Int. J. STD AIDS 2016, 27, 251-267, doi:10.1177/0956462415615443.

6. Hokynar, K.; Korhonen, S.; Norja, P.; Paavonen, J.; Puolakkainen, M. Antibody to Chlamydia trachomatis proteins, TroA and HtrA, as a biomarker for Chlamydia trachomatis infection. Eur J. Clin. Microbiol Infect. Dis 2017, 36, 49-56, doi:10.1007/s10096-016-2769-7.

7. Wang, S.P.; Grayston, J.T. Immunologic relationship between genital TRIC, Lymphogranuloma venereum, and related organisms in a new microtiter indirect immunofluorescence test. Am. J. Ophthalmol. 1970, 70, 367-374.

8. Morre, S.A.; Munk, C.; Persson, K.; Kruger-Kjae S.; van Dijk, R.; Meijer, C.J.; van Den Brule, A.J. Comparison of three commercially available peptide-based immunoglobulin $\mathrm{G}$ (IgG) and IgA assays to microimmunofluorescence assay for detection of Chlamydia trachomatis antibodies. J. Clin. Microbiol. 2002, 40, 584-587.

9. Böttcher, M. Chlamydia trachomatis. Information and Notes on Diagnosis. Available online: http:// www.medac-diagnostika.de/index.php?id product $=3526 \&$ controller $=$ product $\&$ id lang=1 (accessed on 11 November 2019).

10. Labsystems diagnostics. Chlamydia trachomatis EIA tests. Available online: https:// www.labsystemsdx.com /specialty/enzymaticimmuno-assays-eia/chlamydia-trachomatiseia-tests (accessed on 11 November 2019).

11. Savyon diagnostics. SeroCT ${ }^{T M}$ RT. Available online: https://www.savyondiagnostics.com/ elisa/ (accessed on 11 November 2019).

12. Wills, G.S.; Horner, P.J.; Reynolds, R.; Johnson, A.M.; Muir, D.A.; Brown, D.W.; Winston, A.; Broadbent, A.J.; Parker, D.; McClure, M.O. Pgp3 antibody enzyme-linked immunosorbent assay, a sensitive and specific assay for seroepidemiological analysis of Chlamydia trachomatis infection. Clin. Vaccine Immunol. 2009, 16, 835-843, doi:10.1128/CVI.00021-09.

13. Horner, P.J.; Wills, G.S.; Righarts, A.; Vieira, S.; Kounali, D.; Samuel, D.; Winston, A.; Muir, D.; Dickson, N.P.; McClure, M.O. Chlamydia trachomatis Pgp3 antibody persists and correlates with self-reported infection and behavioural risks in a blinded cohort study. PLOS ONE 2016, 11, e0151497, doi:10.1371/ journal.pone.0151497.

14. Winstanley, C.E.; Ramsey, K.H.; Marsh, P.; Clarke, I.N. Development and evaluation of an enzyme-linked immunosorbent assay for the detection of antibodies to a common urogenital derivative of Chlamydia trachomatis plasmidencoded PGP3. J. Immunol. Methods 2017, 445, 23-30, doi:10.1016/j.jim.2017.03.002.

15. Migchelsen, S.J.; Martin, D.L.; Southisombath, K.; Turyaguma, P.; Heggen, A.; Rubangakene, P.P.; Joof, H.; Makalo, P.; Cooley, G.; Gwyn, S.; et al. Defining seropositivity thresholds for use in trachoma elimination studies. PLoS Negl. 
Trop. Dis. 2017, 11, e0005230, doi:10.1371/ journal.pntd.0005230.

16. Waterboer, T.; Sehr, P.; Michael, K.M.; Franceschi, S.; Nieland, J.D.; Joos, T.O.; Templin, M.F.; Pawlita, M. Multiplex human papillomavirus serology based on in situpurified glutathione s-transferase fusion proteins. Clin. Chem. 2005, 51, 1845-1853, doi:10.1373/clinchem.2005.052381.

17. Trabert, B.; Waterboer, T.; Idahl, A.; Brenner, N.; Brinton, L.A.; Butt, J.; Coburn, S.B.; Hartge, P.; Hufnagel, K.; Inturrisi, F.; et al. Antibodies against Chlamydia trachomatis and ovarian cancer risk in two independent populations. J. Natl. Cancer Inst. 2018, doi:10.1093/jnci/djy084.

18. Goodhew, E.B.; Priest, J.W.; Moss, D.M.; 2 Zhong, G.; Munoz, B.; Mkocha, H.; Martin, D.L.; West, S.K.; Gaydos, C.; Lammie, P.J. CT694 and pgp3 as serological tools for monitoring trachoma programs. PLoS Negl. Trop. Dis. 2012, 6, e1873.

19. Hufnagel, K.; Lueong, S.; WillhauckFleckenstein, M.; Hotz-Wagenblatt, A.; Miao, 25. B.; Bauer, A.; Michel, A.; Butt, J.; Pawlita, M.; Hoheisel, J.D.; et al. Immunoprofiling of Chlamydia trachomatis using wholeproteome microarrays generated by on-chip in situ expression. Sci. Rep. 2018, 8, 7503, doi:10.1038/s41598-018-25918-3.

20. Hoenderboom, B.M.; van Oeffelen, A.A.; van Benthem, B.H.; van Bergen, J.E.; Dukers- 26. Muijrers, N.H.; Gotz, H.M.; Hoebe, C.J.; Hogewoning, A.A.; van der Klis, F.R.; van Baarle, D.; et al. The Netherlands Chlamydia cohort study (NECCST) protocol to assess the risk of late complications following Chlamydia trachomatis infection in women. BMC Infect. Dis. 2017, 17, 264, doi:10.1186/s12879-017- 27 2376-y.

21. van den Broek, I.V.; van Bergen, J.E.; Brouwers, E.E.; Fennema, J.S.; Gotz, H.M.; Hoebe, C.J.; Koekenbier, R.H.; Kretzschmar, M.; Over, E.A.; Schmid, B.V.; et al. Effectiveness of yearly, register based screening for chlamydia in the 28 . Netherlands: Controlled trial with randomised stepped wedge implementation. BMJ 2012, 345, e4316, doi:10.1136/bmj.e4316.

22. Angenendt, P.; Kreutzberger, J.; Glokler, J.; Hoheisel, J.D. Generation of high density protein microarrays by cell-free in situ expression of unpurified PCR products. Mol. Cell Proteomics 2006, 5, 1658-1666, doi:10.1074/mcp. T600024-MCP200

23. Woodhall, S.C.; Gorwitz, R.J.; Migchelsen, S.J.; Gottlieb, S.L.; Horner, P.J.; Geisler, W.M.; Winstanley, C.; Hufnagel, K.; Waterboer, T.; Martin, D.L.; et al. Advancing the public health applications of Chlamydia trachomatis serology. Lancet Infect. Dis. 2018, 18, e399-e407, doi:10.1016/S1473-3099(18)30159-2.

24. Horner, P.J.; Wills, G.S.; Reynolds, R.; Johnson, A.M.; Muir, D.A.; Winston, A.; Broadbent, A.J.; Parker, D.; McClure, M.O. Effect of time since exposure to Chlamydia trachomatis on chlamydia antibody detection in women: A cross-sectional study. Sex. Transm. Infect. 2013, 89, 398-403.

25. Hoenderboom, B.M.; van Willige, M.E.; Land, J.A.; Pleijster, J.; Gotz, H.M.; van Bergen, J.; Dukers-Muijrers, N.; Hoebe, C.; van Benthem, B.H.B.; Morre, S.A. Antibody testing in estimating past exposure to Chlamydia trachomatis in the Netherlands Chlamydia Cohort Study. Microorganisms 2019, 7 , doi:10.3390/microorganisms 7100442 .

26. Horner, P.J.; Wills, G.S.; Reynolds, R.; Johnson, A.M.; Muir, D.A.; Winston, A.; Broadbent, A.J.; Parker, D.; McClure, M.O. Effect of time since exposure to Chlamydiatrachomatis on chlamydia antibody detection in women: A cross-sectional study. Sex. Transm. Infect. 2013, 89, 398-403, doi:10.1136/sextrans-2011-050386.

27. Ohman, H.; Rantsi, T.; Joki-Korpela, P.; Tiitinen, A.; Surcel, H.M. Prevalence and persistence of Chlamydia trachomatis-specific antibodies after occasional and recurrent infections. Sex. Transm. Infect. 2019, 10.1136/ sextrans-2018-053915.

28. Michel, A.; Waterboer, T.; Kist, M.; Pawlita, M. Helicobacter pylori multiplex serology. 
Helicobacter 2009, 14, 525-535, doi:10.1111/ j.1523-5378.2009.00723.X.

29. Zhong, G.; Fan, P.; Ji, H.; Dong, F.; Huang, Y. Identification of a chlamydial protease-like activity factor responsible for the degradation of host transcription factors. J. Exp. Med. 2001, 193, 935-942, doi:10.1084/jem.193.8.935.

30. Chen, A.L.; Johnson, K.A.; Lee, J.K.; Sutterlin, C.; Tan, M. CPAF: A Chlamydial protease in search of an authentic substrate. PLoS Pathog. 2012, 8, e1002842, doi:10.1371/journal. ppat.1002842.

31. Korhonen, S.; Hokynar, K.; Mannonen, L.; 34 Paavonen, J.; Hiltunen-Back, E.; Puolakkainen, M. Transcriptional expression of the ompA, cpaf, tarp, and tox genes of Chlamydia trachomatis clinical isolates at different stages of the developmental cycle. Microorganisms 2019, 7 , doi:10.3390/microorganisms7060153.

32. Van Ess, E.F.; Eck-Hauer, A.; Land, J.A.;
Morre, S.A.; Ouburg, S. Combining individual Chlamydia trachomatis IgG antibodies MOMP, TARP, CPAF, OMP2, and HSP60 for tubal factor infertility prediction. Am. J. Reprod. Immunol. 2019, 81, e13091, doi:10.1111/aji.13091.

33. van Ess, E.F.; Ouburg, S.; Spaargaren, J.; Land, J.A.; Morre, S.A. Performance of the multitarget Mikrogen Chlamydia trachomatis IgG ELISA in the prediction of tubal factor infertility (TFI) in subfertile women: Comparison with the Medac MOMP IgG ELISA plus. Pathog. Dis. 2017, 75, doi:10.1093/femspd/ftx067.

34. Chen, C.; Chen, D.; Sharma, J.; Cheng, W.; Zhong, Y.; Liu, K.; Jensen, J.; Shain, R.; Arulanandam, B.; Zhong, G. The hypothetical protein CT813 is localized in the Chlamydia trachomatis inclusion membrane and is immunogenic in women urogenitally infected with C. trachomatis. Infect. Immun. 2006, 74, 4826-4840, doi:10.1128/IAI.00081-06. 


\section{Supplementary material}

- $\quad$ Supplementary Figure 1: Composition of serum pools

- $\quad$ Supplementary Methods 1: Calculation and comparison of three different threshold criteria

- Supplementary Table 1: The 120 highest reactive antigens selected from the whole proteome array 


\section{Supplementary Figure 1: Composition of serum pools}

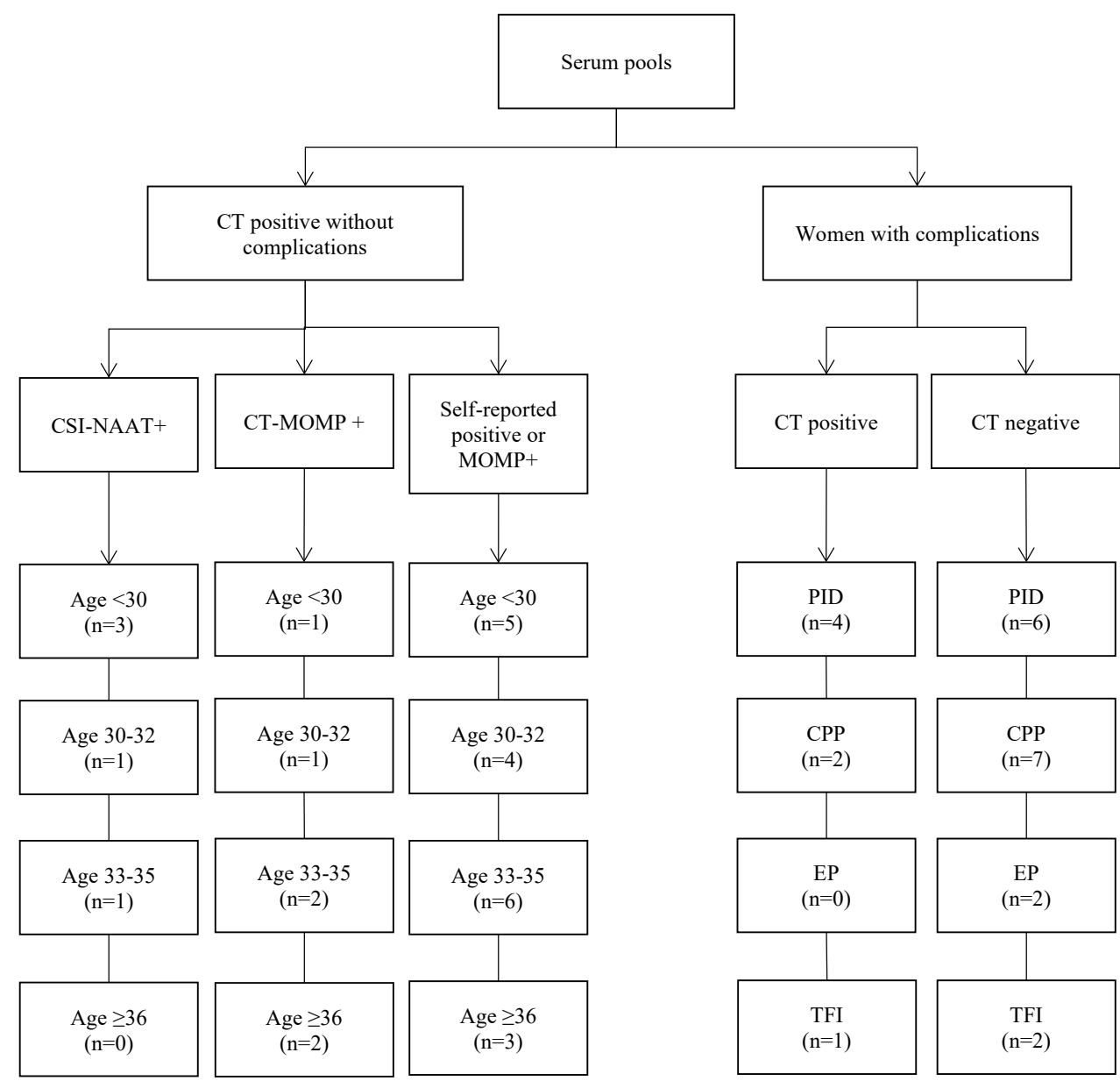

Supplementary Figure 1 Composition of serum pools; CT = Chlamydia trachomatis, NAAT = Nucleic Acid Amplification Test, MOMP = Major Outer Membrane Protein, PID = Pelvic Inflammatory Disease, CPP = chronic pelvic pain, $\mathrm{EP}=$ ectopic pregnancy, $\mathrm{TFI}=$ tubal factor infertility. 


\section{Supplementary Methods 1: Calculation and comparison of three different threshold criteria}

For analysis of the proteome immunoassays, the acquired .gpr-files of all performed immunoassays were imported to $\mathrm{R}$ and different threshold criteria were calculated which are the following: the former used global threshold (Hufnagel et al., 2018) which considers a signal as significant if a MFI of a spot meets the following criterion:

$$
\text { (1) } \mathrm{MFI}_{\text {spot }}>\mathrm{MFIs}_{\text {n.c. }}+5 \sigma \mathrm{MFIs}_{\text {n.c. }}
$$

A second more robust global threshold criterion which utilizes the median (MED) and median absolute deviation (MAD):

(2) $\mathrm{MFI}_{\text {spot }}>\operatorname{MED}\left(\mathrm{MFI}_{\text {n.c. }}\right)+5 \mathrm{MAD}\left(\mathrm{MFI}_{\text {n.c. }}\right)$

And a third approach in which seropositivity was determined by neighborhood averaging, i.e. by calculating a specific threshold for each spot position in order to address local variation of the signal intensity across the array. The threshold criterion takes the relative distances of the spots into account, so that for the calculation of one spot's threshold the MFI values of the 50 closest spots are considered, not distinguishing between negative controls and protein spots but excluding positive controls.

(3) $\mathrm{MFI}_{\text {spot }}>\mathrm{MED}\left(\mathrm{MFI}_{50 \text { closest spots }}\right)+3 \mathrm{MAD}\left(\mathrm{MFI}_{50 \text { closest spots }}\right)$

An antigen is selected if it shows a given threshold-fold on any of the analyzed slides. The antigens were sorted by their maximal observed threshold-fold value and the top 120 antigens of that list are chosen for further analysis. Thereby, the applied threshold-fold criterion for seropositivity is set by the technical restrictions of the single sera analysis. Concerning this selection procedure, the global application of a threshold which is calculated from negative controls located on just one end of the slide might result in a biased selection. Therefore, antigens which are located in an area which possess high intensity signals will be overrepresented in the above described selection list. In order to adjust for this issue individual thresholds were calculated for each spot by neighborhood averaging.

For each spot an individual threshold was calculated according to equation (3), whereas positive controls were excluded from the calculations. The effect of the neighborhood averaging method on threshold-fold data is shown in Figure 1. 

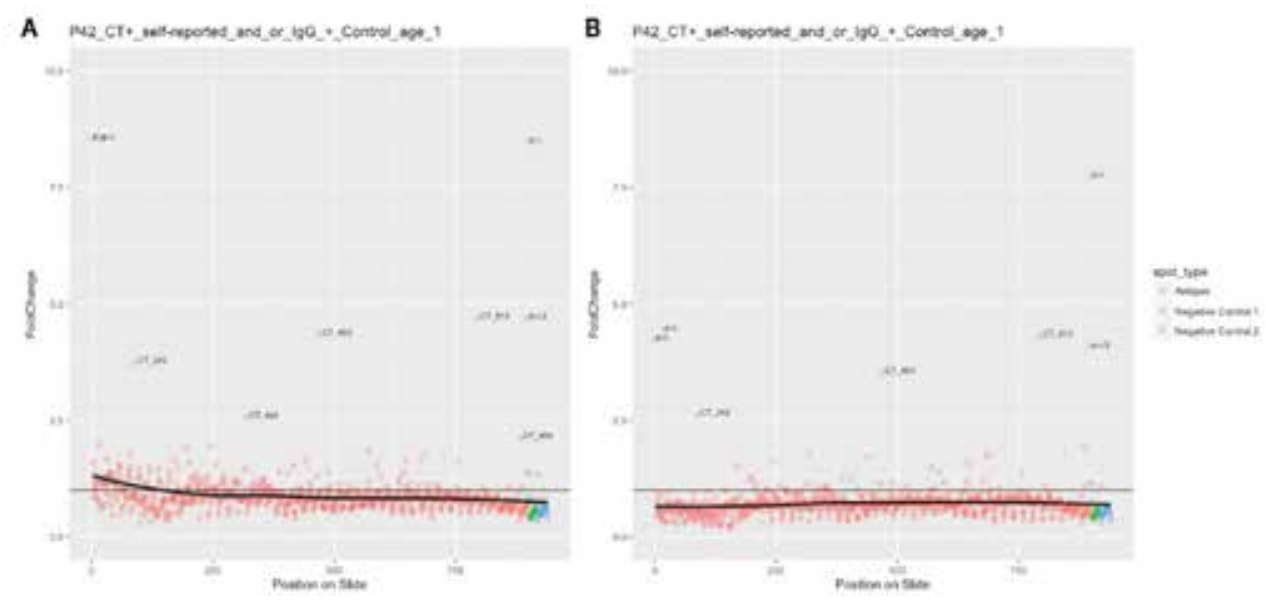

Figure 1: Effects of neighborhood averaging on local signal in-varieties. All MFl-values are foldchange transformed after equation 2 (A) utilizing the median and mad of the negative controls and equation 3 which describes the neighborhood averaging (B) and plotted against the position on the slide (row-wise). Reactive antigens were labeled when they exceeded the fold-change of 1.7 which was chosen for illustration concerns of the plot.

In Figure $1 \mathrm{~A}$, the threshold-fold data calculated by the traditional method shows a gradient from the top to the bottom of the slide. After applying neighborhood averaging to the data (Figure $1 \mathrm{~B}$ ), the horizontal gradient disappeared. Selection lists were created using the global threshold and the spot individual neighborhood averaging method (see Supplementary Table 1). Both antigen lists were sorted by the maximal observed threshold-fold. The overlap between both lists was calculated and hits $51.67 \%$ at a list length of 120 . The comparison of the selection list gained by applying a global threshold and by using the neighborhood method revealed that antigens with high reactivities are shared by both lists. The major differences lie within the lower ends of the lists which comprise reactivities that are slightly above threshold. Based on the knowledge of the presence of local signal in-varieties, the list generated from the neighborhood averaging was chosen to be used when selecting the 120 antigens based on serum pool incubations on whole-proteome microarrays. 
Supplementary Table 1: The 120 most reactive antigens

\begin{tabular}{|c|c|c|c|c|c|c|c|c|}
\hline \multicolumn{5}{|c|}{ Global Threshold } & \multicolumn{4}{|c|}{ Neighborhood Averaging } \\
\hline Rank & Antigen & $\max$ & mean & sd & ID & $\max$ & mean & sd \\
\hline 1 & CT_813 & 7.282 & 1.401 & 1.469 & CT_813 & 11.096 & 2.114 & 2.091 \\
\hline 2 & CT_463 & 6.362 & 1.053 & 1.202 & CT_463 & 8.670 & 1.723 & 1.761 \\
\hline 3 & CT_242 & 4.830 & 1.004 & 0.943 & CT_242 & 8.488 & 1.371 & 1.482 \\
\hline 4 & CT_123 & 3.889 & 0.626 & 0.902 & CT_123 & 7.437 & 1.001 & 1.670 \\
\hline 5 & CT 456 & 3.253 & 0.735 & 0.671 & CT_336 & 6.547 & 0.953 & 0.958 \\
\hline 6 & CT_183 & 2.850 & 0.647 & 0.444 & CT_326 & 6.110 & 1.008 & 0.889 \\
\hline 7 & CT_442 & 2.552 & 0.691 & 0.498 & CT_351 & 5.851 & 0.936 & 0.828 \\
\hline 8 & CT_868 & 2.419 & 0.664 & 0.480 & CT_082 & 5.591 & 0.981 & 0.830 \\
\hline 9 & pGP3 & 2.377 & 0.486 & 0.452 & CT_856 & 4.979 & 0.885 & 0.681 \\
\hline 10 & CT_732 & 2.369 & 0.648 & 0.509 & CT_456 & 4.691 & 1.144 & 1.015 \\
\hline 11 & CT_858 & 2.363 & 0.662 & 0.464 & pGP3 & 4.375 & 0.726 & 0.672 \\
\hline 12 & CT_759 & 2.181 & 0.684 & 0.474 & CT_732 & 4.272 & 0.873 & 0.742 \\
\hline 13 & CT_618 & 2.157 & 0.603 & 0.369 & CT_057 & 4.008 & 0.828 & 0.579 \\
\hline 14 & CT_555 & 2.071 & 0.498 & 0.316 & CT_744 & 3.850 & 0.844 & 0.665 \\
\hline 15 & CT_040 & 2.039 & 0.485 & 0.312 & CT_414 & 3.826 & 0.925 & 0.628 \\
\hline 16 & $\mathrm{CT}_{-}^{-} 746$ & 2.003 & 0.492 & 0.371 & CT_778 & 3.745 & 0.753 & 0.534 \\
\hline 17 & CT_117 & 1.987 & 0.467 & 0.366 & CT_027 & 3.730 & 1.111 & 0.611 \\
\hline 18 & CT_467 & 1.975 & 0.655 & 0.457 & CT_555 & 3.673 & 0.838 & 0.656 \\
\hline 19 & CT_027 & 1.927 & 0.675 & 0.417 & CT_183 & 3.668 & 1.053 & 0.677 \\
\hline 20 & CT_822 & 1.920 & 0.464 & 0.336 & CT_166 & 3.540 & 0.850 & 0.528 \\
\hline 21 & CT_116 & 1.914 & 0.618 & 0.349 & CT_147 & 3.447 & 0.661 & 0.574 \\
\hline 22 & CT_218 & 1.908 & 0.586 & 0.408 & CT_618 & 3.288 & 0.960 & 0.484 \\
\hline 23 & $\mathrm{CT}_{-} 147$ & 1.901 & 0.481 & 0.365 & CT_105 & 3.192 & 0.749 & 0.429 \\
\hline 24 & CT_229 & 1.895 & 0.622 & 0.391 & CT_467 & 3.184 & 1.098 & 0.697 \\
\hline 25 & CT_639 & 1.818 & 0.451 & 0.330 & CT_858 & 3.107 & 0.972 & 0.563 \\
\hline 26 & CT_249 & 1.807 & 0.640 & 0.369 & CT_116 & 3.088 & 0.858 & 0.434 \\
\hline 27 & CT_001 & 1.806 & 0.509 & 0.322 & CT_825 & 3.073 & 0.777 & 0.564 \\
\hline 28 & CT_115 & 1.776 & 0.567 & 0.353 & CT_381 & 3.044 & 0.862 & 0.430 \\
\hline 29 & CT_346 & 1.695 & 0.424 & 0.284 & CT_751 & 2.912 & 0.674 & 0.397 \\
\hline 30 & CT_579 & 1.659 & 0.586 & 0.317 & CT_333 & 2.865 & 0.668 & 0.414 \\
\hline 31 & CT_584 & 1.656 & 0.572 & 0.379 & CT_759 & 2.854 & 0.927 & 0.560 \\
\hline 32 & CT_795 & 1.652 & 0.593 & 0.276 & CT_720 & 2.824 & 0.787 & 0.403 \\
\hline 33 & CT_307 & 1.631 & 0.537 & 0.284 & CT_019 & 2.794 & 0.575 & 0.501 \\
\hline 34 & CT_703 & 1.596 & 0.519 & 0.292 & CT_372 & 2.774 & 0.660 & 0.377 \\
\hline 35 & CT_541 & 1.579 & 0.475 & 0.314 & CT_681 & 2.723 & 0.970 & 0.357 \\
\hline 36 & CT_181 & 1.570 & 0.581 & 0.333 & CT_708 & 2.695 & 0.700 & 0.391 \\
\hline 37 & CT_228 & 1.562 & 0.320 & 0.276 & CT_639 & 2.675 & 0.725 & 0.597 \\
\hline 38 & CT_381 & 1.557 & 0.592 & 0.314 & CT_866 & 2.665 & 0.711 & 0.364 \\
\hline 39 & CT_814 & 1.556 & 0.588 & 0.286 & CT_458 & 2.635 & 0.685 & 0.354 \\
\hline 40 & CT_048 & 1.543 & 0.419 & 0.301 & CT_796 & 2.634 & 0.884 & 0.384 \\
\hline 41 & CT_313 & 1.539 & 0.453 & 0.282 & CT_746 & 2.609 & 0.778 & 0.430 \\
\hline 42 & CT_741 & 1.523 & 0.611 & 0.297 & CT_529 & 2.587 & 1.019 & 0.572 \\
\hline 43 & CT_372 & 1.523 & 0.409 & 0.260 & CT_205 & 2.535 & 0.841 & 0.365 \\
\hline 44 & CT_414 & 1.518 & 0.626 & 0.281 & CT_442 & 2.517 & 0.937 & 0.491 \\
\hline
\end{tabular}




\begin{tabular}{|c|c|c|c|c|c|c|c|c|}
\hline \multicolumn{5}{|c|}{ Global Threshold } & \multicolumn{4}{|c|}{ Neighborhood Averaging } \\
\hline Rank & Antigen & $\max$ & mean & sd & ID & $\max$ & mean & sd \\
\hline 45 & CT_388 & 1.517 & 0.554 & 0.313 & CT_579 & 2.517 & 0.790 & 0.416 \\
\hline 46 & CT_681 & 1.515 & 0.585 & 0.276 & CT_587 & 2.442 & 0.604 & 0.383 \\
\hline 47 & CT_232 & 1.495 & 0.420 & 0.291 & CT_664 & 2.435 & 0.687 & 0.368 \\
\hline 48 & CT_567 & 1.490 & 0.578 & 0.292 & CT_4 418 & 2.400 & 0.793 & 0.304 \\
\hline 49 & CT_802 & 1.485 & 0.510 & 0.277 & CT_857 & 2.374 & 0.836 & 0.339 \\
\hline 50 & CT_526 & 1.482 & 0.428 & 0.273 & CT_231 & 2.314 & 0.761 & 0.357 \\
\hline 51 & CT_691 & 1.480 & 0.411 & 0.282 & CT_040 & 2.302 & 0.753 & 0.350 \\
\hline 52 & CT_110 & 1.475 & 0.465 & 0.251 & CT_249 & 2.253 & 0.860 & 0.434 \\
\hline 53 & CT_659 & 1.473 & 0.443 & 0.273 & CT_868 & 2.250 & 0.965 & 0.464 \\
\hline 54 & CT_853 & 1.466 & 0.524 & 0.290 & CT_802 & 2.244 & 0.666 & 0.294 \\
\hline 55 & CT_300 & 1.465 & 0.519 & 0.280 & CT_398 & 2.238 & 0.762 & 0.386 \\
\hline 56 & CT_009 & 1.460 & 0.444 & 0.261 & CT_096 & 2.237 & 0.571 & 0.416 \\
\hline 57 & CT_081 & 1.453 & 0.579 & 0.270 & $\mathrm{CT}_{-}^{-} 762$ & 2.226 & 0.714 & 0.339 \\
\hline 58 & CT_353 & 1.447 & 0.526 & 0.286 & CT_218 & 2.211 & 0.934 & 0.415 \\
\hline 59 & CT_601 & 1.438 & 0.476 & 0.325 & CT_682 & 2.203 & 0.769 & 0.364 \\
\hline 60 & CT_830 & 1.418 & 0.500 & 0.265 & CT_118 & 2.180 & 0.944 & 0.417 \\
\hline 61 & CT_844 & 1.413 & 0.454 & 0.331 & CT_532 & 2.167 & 0.658 & 0.320 \\
\hline 62 & CT_482 & 1.408 & 0.570 & 0.299 & CT_111 & 2.164 & 0.618 & 0.295 \\
\hline 63 & CT_330 & 1.408 & 0.465 & 0.309 & CT_741 & 2.136 & 0.823 & 0.270 \\
\hline 64 & CT_186 & 1.403 & 0.396 & 0.264 & CT_346 & 2.135 & 0.661 & 0.282 \\
\hline 65 & CT_067 & 1.399 & 0.425 & 0.229 & CT_872 & 2.113 & 0.694 & 0.310 \\
\hline 66 & CT_398 & 1.385 & 0.511 & 0.285 & CT_822 & 2.030 & 0.701 & 0.324 \\
\hline 67 & CT_159 & 1.380 & 0.495 & 0.266 & CT_603 & 2.016 & 0.663 & 0.373 \\
\hline 68 & CT_597 & 1.376 & 0.530 & 0.259 & CT_229 & 1.955 & 0.826 & 0.463 \\
\hline 69 & CT_458 & 1.370 & 0.537 & 0.269 & CT_601 & 1.954 & 0.641 & 0.369 \\
\hline 70 & CT_751 & 1.369 & 0.411 & 0.269 & CT_190 & 1.951 & 0.748 & 0.309 \\
\hline 71 & CT_602 & 1.363 & 0.488 & 0.272 & CT_701 & 1.940 & 0.770 & 0.299 \\
\hline 72 & CT_569 & 1.361 & 0.544 & 0.259 & CT_313 & 1.936 & 0.712 & 0.253 \\
\hline 73 & CT_503 & 1.361 & 0.416 & 0.258 & $\mathrm{CT}_{-}^{-} 110$ & 1.894 & 0.689 & 0.257 \\
\hline 74 & CT_325 & 1.360 & 0.411 & 0.273 & CT_841 & 1.821 & 0.796 & 0.270 \\
\hline 75 & CT_756 & 1.359 & 0.384 & 0.268 & CT_226 & 1.802 & 0.808 & 0.231 \\
\hline 76 & CT_789 & 1.358 & 0.598 & 0.274 & CT_224 & 1.799 & 0.686 & 0.220 \\
\hline 77 & CT_657 & 1.355 & 0.394 & 0.276 & CT_706 & 1.710 & 0.619 & 0.260 \\
\hline 78 & CT_143 & 1.352 & 0.553 & 0.313 & CT_260 & 1.675 & 0.643 & 0.245 \\
\hline 79 & CT_587 & 1.333 & 0.397 & 0.273 & CT_545 & 1.662 & 0.694 & 0.274 \\
\hline 80 & CT_538 & 1.321 & 0.418 & 0.245 & CT_541 & 1.661 & 0.614 & 0.358 \\
\hline 81 & $\mathrm{CT}^{-}$129 & 1.320 & 0.474 & 0.261 & CT_842 & 1.640 & 0.746 & 0.243 \\
\hline 82 & $\mathrm{CT}_{-}^{-} 118$ & 1.318 & 0.629 & 0.320 & $\mathrm{CT}_{-}^{-} 143$ & 1.636 & 0.814 & 0.303 \\
\hline 83 & CT_603 & 1.314 & 0.480 & 0.281 & CT_228 & 1.629 & 0.441 & 0.286 \\
\hline 84 & CT_480 & 1.307 & 0.432 & 0.242 & CT_799 & 1.601 & 0.670 & 0.227 \\
\hline 85 & CT_421 & 1.306 & 0.502 & 0.258 & CT_795 & 1.601 & 0.800 & 0.283 \\
\hline 86 & CT_772 & 1.300 & 0.386 & 0.280 & CT_480 & 1.586 & 0.595 & 0.254 \\
\hline 87 & CT_ 444 & 1.299 & 0.403 & 0.267 & CT_331 & 1.571 & 0.708 & 0.219 \\
\hline 88 & $\mathrm{CT}_{-}^{-} 173$ & 1.286 & 0.537 & 0.253 & CT_547 & 1.570 & 0.746 & 0.280 \\
\hline 89 & CT_724 & 1.279 & 0.540 & 0.255 & CT_273 & 1.534 & 0.506 & 0.250 \\
\hline
\end{tabular}




\begin{tabular}{|c|c|c|c|c|c|c|c|c|}
\hline \multicolumn{5}{|c|}{ Global Threshold } & \multicolumn{4}{|c|}{ Neighborhood Averaging } \\
\hline Rank & Antigen & $\max$ & mean & sd & ID & $\max$ & mean & sd \\
\hline 90 & CT_829 & 1.273 & 0.436 & 0.245 & CT_827 & 1.520 & 0.646 & 0.256 \\
\hline 91 & CT_532 & 1.271 & 0.517 & 0.308 & CT_117 & 1.506 & 0.604 & 0.308 \\
\hline 92 & CT_449 & 1.269 & 0.558 & 0.249 & CT_382 & 1.478 & 0.724 & 0.230 \\
\hline 93 & CT_568 & 1.265 & 0.435 & 0.274 & CT_703 & 1.459 & 0.765 & 0.230 \\
\hline 94 & CT_433 & 1.265 & 0.431 & 0.265 & CT_724 & 1.458 & 0.769 & 0.177 \\
\hline 95 & CT_342 & 1.261 & 0.505 & 0.227 & CT_446 & 1.438 & 0.718 & 0.199 \\
\hline 96 & CT_231 & 1.258 & 0.488 & 0.297 & CT_821 & 1.429 & 0.381 & 0.254 \\
\hline 97 & CT_457 & 1.257 & 0.477 & 0.219 & CT_089 & 1.421 & 0.396 & 0.260 \\
\hline 98 & CT_529 & 1.257 & 0.574 & 0.329 & CT_004 & 1.418 & 0.660 & 0.213 \\
\hline 99 & CT_446 & 1.256 & 0.554 & 0.277 & CT_307 & 1.404 & 0.797 & 0.217 \\
\hline 100 & СT_832 & 1.254 & 0.427 & 0.275 & CT_609 & 1.388 & 0.705 & 0.198 \\
\hline 101 & CT_080 & 1.250 & 0.432 & 0.272 & CT_292 & 1.371 & 0.588 & 0.203 \\
\hline 102 & CT_841 & 1.244 & 0.530 & 0.275 & CT_544 & 1.367 & 0.601 & 0.221 \\
\hline 103 & CT_357 & 1.243 & 0.398 & 0.261 & CT_022 & 1.358 & 0.658 & 0.154 \\
\hline 104 & CT_281 & 1.240 & 0.470 & 0.268 & CT_812 & 1.352 & 0.591 & 0.304 \\
\hline 105 & CT_847 & 1.235 & 0.459 & 0.258 & CT_115 & 1.336 & 0.712 & 0.239 \\
\hline 106 & CT_539 & 1.234 & 0.420 & 0.230 & CT_482 & 1.322 & 0.731 & 0.188 \\
\hline 107 & CT_528 & 1.234 & 0.519 & 0.260 & CT_325 & 1.317 & 0.528 & 0.247 \\
\hline 108 & CT_797 & 1.234 & 0.423 & 0.243 & CT_277 & 1.315 & 0.517 & 0.259 \\
\hline 109 & CT_076 & 1.234 & 0.480 & 0.287 & CT_341 & 1.308 & 0.642 & 0.173 \\
\hline 110 & CT_440 & 1.232 & 0.480 & 0.259 & CT_452 & 1.302 & 0.559 & 0.264 \\
\hline 111 & CT_737 & 1.231 & 0.384 & 0.251 & CT_871 & 1.302 & 0.603 & 0.179 \\
\hline 112 & CT_481 & 1.228 & 0.484 & 0.244 & CT_342 & 1.301 & 0.685 & 0.173 \\
\hline 113 & CT_277 & 1.220 & 0.384 & 0.270 & CT_678 & 1.284 & 0.664 & 0.166 \\
\hline 114 & CT_212 & 1.216 & 0.481 & 0.227 & CT_162 & 1.283 & 0.724 & 0.188 \\
\hline 115 & CT_192 & 1.213 & 0.390 & 0.225 & CT_121 & 1.280 & 0.632 & 0.225 \\
\hline 116 & CT_612 & 1.211 & 0.460 & 0.239 & CT_521 & 1.273 & 0.672 & 0.178 \\
\hline 117 & CT_873 & 1.210 & 0.465 & 0.310 & CT_388 & 1.268 & 0.686 & 0.213 \\
\hline 118 & CT_563 & 1.209 & 0.542 & 0.269 & CT_468 & 1.268 & 0.713 & 0.167 \\
\hline 119 & CT_272 & 1.209 & 0.449 & 0.239 & CT_172 & 1.256 & 0.699 & 0.168 \\
\hline 120 & CT_172 & 1.208 & 0.512 & 0.237 & CT_474 & 1.253 & 0.613 & 0.218 \\
\hline
\end{tabular}

Max = maximum MFI value, mean = mean MFI value, $\mathrm{SD}=$ standard deviation, $\mathrm{CT}=$ Chlamydia trachomatis . 


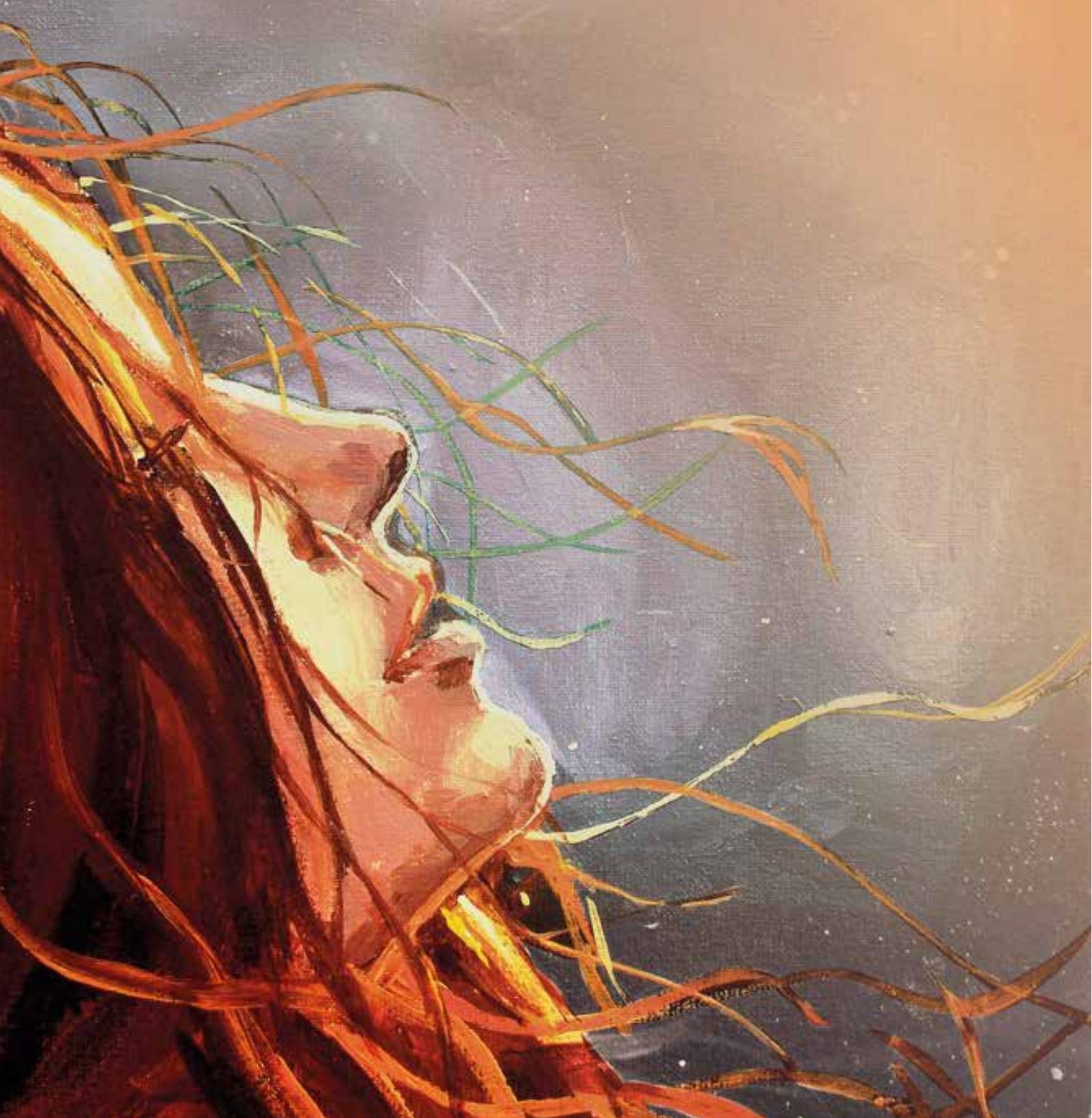




\section{Part 3}

Long-term complications of chlamydla infections and predictive factors 


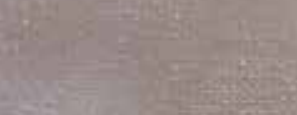

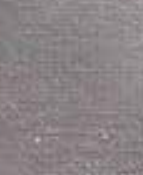

\section{sisting}

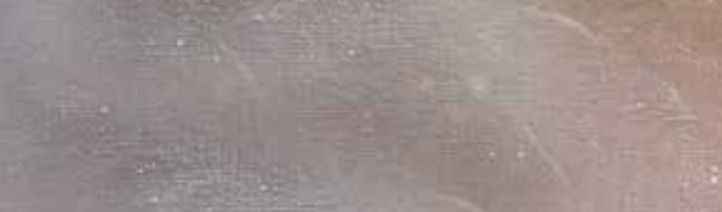

जince

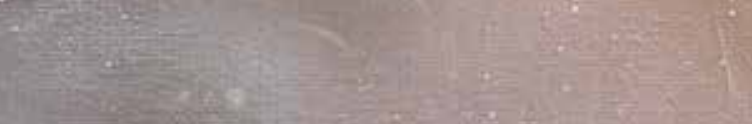

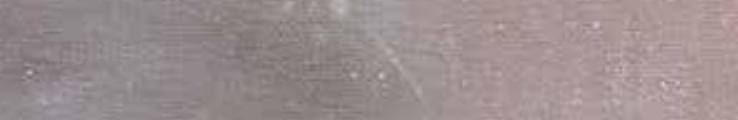

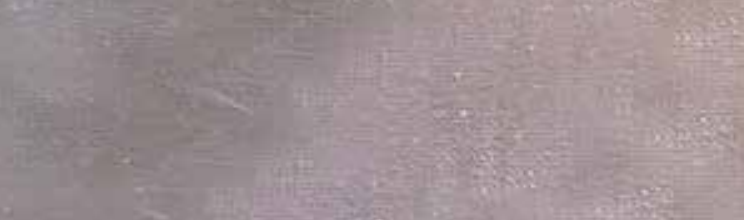

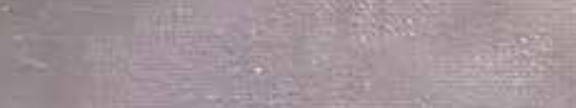

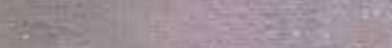

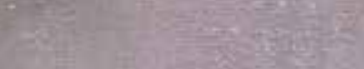

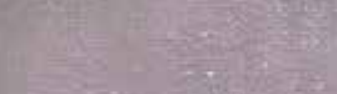

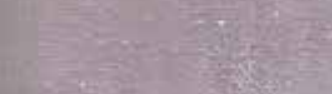

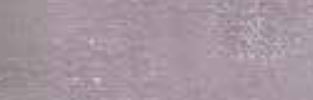

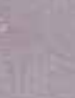

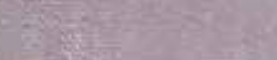

netwo

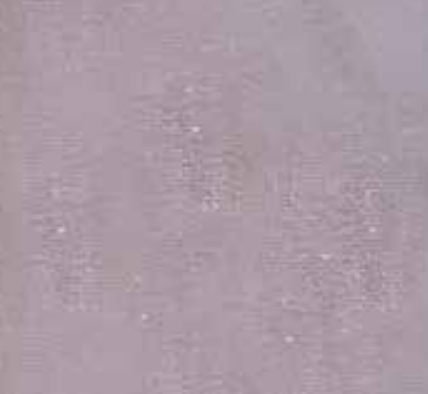

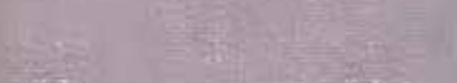

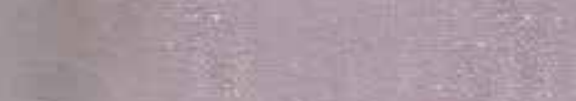




\section{7}

Relation between Chlamydia trachomatis infection and pelvic inflammatory disease, ectopic pregnancy. and tubal factor infertility in a Dutch cohort of women previously tested for chlamydia in a chlamydia screening trial

Bernice M Hoenderboom, Birgit HB van Benthem, Jan EAM van Bergen, Nicole HTM DukersMuijrers, Hannelore M Götz, Christian JPA Hoebe, Arjan A Hogewoning, Jolande A Land, Marianne $A B$ van der Sande, Servaas A Morré, Ingrid VF van den Broek 


\section{Abstract}

Objectives: A better understanding of Chlamydia trachomatis infection (chlamydia) -related sequelae can provide a framework for effective chlamydia control strategies. The objective of this study was to estimate risks and risk factors of pelvic inflammatory disease (PID), ectopic pregnancy, and tubal factor infertility (TFI) with a follow-up time of up until 8 years in women previously tested for chlamydia in the Chlamydia Screening Implementation study (CSI) and participating in the Netherlands Chlamydia Cohort Study (NECCST).

Methods: Women who participated in the CSI (2008-2011, $n=13,498)$, were invited in 2015-2016 for NECCST. Chlamydia positive was defined as a positive CSI-PCR test, positive chlamydia serology and/or self-reported infection (time-dependent). Data on PID, ectopic pregnancy, and TFI were collected by self-completed questionnaires. Incidence rates and hazards ratios were compared between chlamydia positive and chlamydia negative women corrected for confounders.

Results: Of 5704 women included, 29.5\% (95\% Cl 28.3-30.7) were chlamydia-positive. The incidence rate of PID was 1.8 per 1000 person-years (py) (1.6-2.2) overall, 4.4 per 1000py (3.35.7) among chlamydia positives compared to 1.4 per 1000py (1.1-1.7) for chlamydia negatives. For TFI this was 0.4 per 1000py (0.3-0.5) overall, 1.3 per 1000py (0.8-2.1) and 0.2 per 1000py (0.1-0.4) among chlamydia positives and negatives, respectively. And for ectopic pregnancy this was 0.6 per 1000py (0.5-0.8) overall, 0.8 per 1000py (0.4-1.5) and 0.6 per 1000py (0.4-0.8) for chlamydia negatives. Among chlamydia-positive women, the strongest risk factor for PID was symptomatic versus asymptomatic infection (aHR 2.88, 1.4-4.5) and for TFI age $<20$ versus $>24$ years at first infection (HR 4.35, 1.1-16.8).

Conclusion: We found a considerably higher risk for PID and TFI in chlamydia-positive women, but the incidence for ectopic pregnancy was comparable between chlamydia positive and negative women. Overall, the incidence rates of sequelae remained low. 


\section{Background}

Chlamydia trachomatis infection (chlamydia) prevalence has remained high with an estimated annual number of infections of 130 million worldwide in 2012(1). Chlamydia disproportionately affects women of reproductive age with a prevalence of $4.0 \%$ compared to $2.8 \%$ among men(1). Studies indicate that $10-30 \%$ of women experience one or more chlamydia episodes(2, 3). With up to $70 \%$ of infections being asymptomatic, many women are left untreated and are prone to chlamydia-related sequelae(4) such as pelvic inflammatory disease (PID), ectopic pregnancy, and tubal factor infertility (TFI)(5).

Proportions of PID following chlamydia were found between $3.0 \%$ to $30.0 \%(6-10)$, for ectopic pregnancy between $0.2 \%$ to $2.7 \%(7,9-11)$ and for TFI between 0.1 and $6.0 \%(6,7,12)$. Questions remain concerning true risks of sequelae due to study limitations, such as small sample size or limited follow-up time(13), unavoidable misclassification of chlamydia status as it is primarily based on incident (NAAT) tests (9), or lack of information on potential confounders such as sexual risk behaviour, demographic data, and lifestyle factors in cohort studies based on large medical databases(10). These limitations need to be addressed to determine true risks of chlamydiarelated sequelae.

The only way to interrupt the course of a chlamydia infection is to test and treat women at risk for chlamydia. However, there is no scientific evidence that screening decreases prevalence $(2,14$, 15). It might be more effective to move towards targeted screening of women at highest risk for developing sequelae since the clinical course of chlamydia differs greatly between individuals and depends on pathogen factors, environmental factors, and host factors (9, 16-19). To study chlamydia-related sequelae and risk factors, the Netherlands Chlamydia Cohort Study (NECCST) was initiated in 2015. NECCST is an ongoing cohort study among Dutch women of reproductive age and a follow-up study of the 2008-2011 Chlamydia Screening Implementation programme (CSI) in which all women were tested for chlamydia(20). We estimated risks of PID, ectopic pregnancy, and TFI up to eight years after chlamydia infection in women taking into account sexual risk behaviour, demographics and lifestyle factors. To identify women with high risk for sequelae, potential risk factors were evaluated.

\section{Methods}

\section{Study design and participants}

NECCST is a cohort of women of reproductive age to be followed until 2022. The design has been described previously(20). Briefly, women between 16-29 years participated in 2008-2011 in the CSI(14). CSI participants were tested for chlamydia via PCR test $\geq 1$ with a maximum of four times in a four year period. In case of chlamydia positive PCR outcomes participants were referred to the GP or STI clinic for treatment. CSI women were traced in municipal registers and invited for participation in NECCST, Supplementary Figure S1. Participants provided online informed consent to comply with study procedures for collection of questionnaires and biological 
samples. This study was approved by the Medical Ethical Committee Noord-Holland in Alkmaar, the Netherlands (NL 51553.094.14/M014-042).

\section{Procedures}

In 2015-2016, women were invited and informed by regular mail and email. The first NECCST data collection moment included an electronic questionnaire followed by a test kit for self-collection of blood via a finger-prick for chlamydia lgG analyses as a marker for previous infection. The initial NECCST-questionnaire retrospectively inquired about previous chlamydia infections, PID, ectopic pregnancy, and TFI. Additionally, the NECCST-questionnaire addressed demographic factors, sexual behaviour, other STIs, contraceptive use and health characteristics. For all events timing in calendar year was asked. This was done to reconstruct a timeline for time-to-event analysis. Subsequently, CSI data about chlamydia PCR results, self-reported chlamydia infections and age of sexual debut acquired via the CSI-questionnaires were merged with NECCST data.

Chlamydia IgG antibodies were determined in self-collected capillary blood samples, collected in tubes [BD Microtainer SST, USA] and returned to the laboratory via mail. Serum samples were stored at $-20^{\circ} \mathrm{C}$ until thawed for ELISA assay with a sensitivity of $71 \%$ and specificity of $97 \%$ (Medac CT IgG ELISA plus, Wedel, Germany) $(21,22)$.

\section{Definitions and outcomes}

Chlamydia positivity was defined as a positive PCR-test outcome in the CSI study (CSI-PCR+), and/or the presence of chlamydia IgG in serum and/or a self-reported chlamydia infection. From the year of first chlamydia infection onwards, a woman was classified as chlamydia-positive and remained positive. If year of first infection was unknown, i.e. in women with a positive chlamydia IgG result only, multiple imputations were done for the Cox model analyses using truncated regression with 15 simulation datasets(23) to estimate time of first chlamydia infection following sexual debut based on available data from women with a known year of first infection. Women were classified as chlamydia-negative when they were CSI-PCR negative, had no chlamydia antibodies, and did not report a previous infection.

The primary outcomes were PID, ectopic pregnancy, and TFI:

1. PID was defined as a self-reported episode of inflammation of the ovaries, uterus and/or fallopian tubes, diagnosed by a medical professional. To obtain more information, women were asked whether the diagnosis was based on reported symptoms, physical examination, laboratory testing (either blood or vaginal swab examination), laparoscopy or whether this was unknown to them. Furthermore, we asked if women had been admitted to the hospital for the PID episode.

2. Ectopic pregnancy was defined as a self-reported extra uterine pregnancy. Women who were/had been pregnant were asked if they had experienced an ectopic pregnancy, and if so, the number and year of ectopic pregnancies.

3. TFI was defined as self-reported infertility caused by abnormalities of the tubes (possibly combined with other causes of infertility), diagnosed by a medical professional. All women who reported infertility were asked which fertility examinations had been conducted, i.e. 
chlamydia antibody testing (CAT), hysterosalpingography (HSG), contrast ultrasonography, laparoscopy or 'none of the above'. Although self-reported TFI was found a reliable measure of TFI in the Netherlands (24) we validated a subset of self-reported TFI cases. Substantial agreement (Kappa value 0.73) between self-reported TFI and medical registers was found in the first NECCST-round.

Exposure time in time-to-event analyses was defined as the total number of person-years, starting from when a woman became sexually active (or minimum age defined at 12 years for earlier reports). Exposure time ended at last data collection point or when a specific outcome event occurred.

\section{Statistical analyses}

Incidence rates (calculated as the number of new outcomes divided by total person-years at risk) of primary study endpoints PID, ectopic pregnancy, and TFI were described.

The association between chlamydia status and each of the primary study endpoints was retrospectively assessed through Kaplan-Meier curves and univariable and multivariable Cox Proportional Hazards regression models. Risks were expressed in hazard ratios (HR). Chlamydia status was included as a time-dependent variable. The following variables were considered potential confounders and included in the model if there was $a \geq 10 \%$ change in the regression coefficient: age (time-varying), gonorrhoea infection, migration background (Western vs non-Western, based on parental country of birth), educational level low/middle versus high (i.e. low/medium: no education, primary education only, lower general secondary education, and vocational education; high level: all other education levels), lifetime sex partners, age of sexual debut, condom use with casual partner, smoking behaviour, and Intrauterine Device (IUD) insertion. The proportional-hazard assumption was checked using log-log plots and testing Schoenfeld residuals.

To be able to identify chlamydia-positive women with a high risk for sequelae risk factors for PID and TFI were determined by Cox regression models. Included risk factors were demopgraphics, sexual risk behaviour, contraceptive use, chlamydia infection variables, and serology (positive/ negative outcome). All variables were assessed via univariable analysis and those associated $(p<0.10)$ with the outcome were entered via backward stepwise selection into the multivariable model.

To establish how closely risk factors were associated with the outcomes regardless of chlamydia status, factors were univariably tested with each outcome.

Multiple imputations and analyses were performed in STATA (version 14; StataCorp, College Station, TX, USA).

\section{Sensitivity analyses}

First, we repeated analyses without multiple imputations for women with an unknown year of chlamydia-infection. Second, analyses were repeated including only women with CSI-PCRpositive test results, because CSI-PCR results have high sensitivity and specificity and no recall bias. Third, repeated analyses for ectopic pregnancy and TFI were restricted to women who had been pregnant at least once or who had ever tried to become pregnant. Fourth, due to residual 
uncertainties in PID diagnosis, we repeated analyses for PID including only hospitalised cases and for PID cases that occurred later than year of first chlamydia infection. This was not indicated for ectopic pregnancy and TFI since for both only one case occurred in the same year as the first chlamydia-infection.

\section{Results}

Between November 2015-August 2016, 13,498/14,865 (91.9\%) of eligible CSI women were able to be retraced and invited for NECCST. 5,704 (42.3\%) women were enrolled and completed the initial questionnaire, Supplementary Figure S1. In total, 1,682 (29.5\%, 95\% Cl 28.3-30.7) women were ever chlamydia-positive and 4,022 (70.5\%, 95\% $0.69-0.72)$ had remained chlamydia-negative. Chlamydia positivity was based on 1,469 (87.3\%) self-reported infections, of which 341 (23.2\%) also tested PCR-positive during CSI, and 360 (24.5\%) also tested positive for chlamydia IgG. Chlamydia antibody testing was done in 3,670 (65\%) women of whom 15.5\% (95\%Cl 14.3-16.7) was positive. The remaining chlamydia positives $(n=213)$ only had a positive chlamydia lgG test $(n=208)$, were only PCR-positive in CSI $(n=4)$ or were both chlamydia IgG- and PCR-positive $(n=1)$ but did not report this, Supplementary Figure S2. Multiple imputations to impute time for first chlamydia were used for 226/1682 (13.4\%) chlamydia-positive women. These women were slightly older (31.6 vs. 30.8 years) and had less lifetime partners and more condom use compared to chlamydia positives with known date of infection. Mean age at start of exposure time (sexual debut) was 16.9 (SD 2.4) years and mean exposure time was 14.2 (SD 3.9) years. Total exposure time was 86,610 person-years. Characteristics of study participants are presented in table 1 . 
Table 1. Characteristics of the study population by chlamydia status at start of NECCST.

\begin{tabular}{|c|c|c|c|c|}
\hline & $\begin{array}{l}\text { Overall } \\
\mathrm{n}(\%)\end{array}$ & $\begin{array}{l}\text { Chlamydia-negative } \\
\mathrm{n}(\%)\end{array}$ & $\begin{array}{l}\text { Chlamydia-positive } \\
\mathrm{n}(\%)\end{array}$ & p-value \\
\hline & $5704(100 \%)$ & $4022(70.5 \%)$ & $1682(29.5 \%)$ & \\
\hline Age (years) mean (SD) & $31.1(3.8)$ & $31.2(3.8)$ & $30.9(3.8)$ & $0.003^{*}$ \\
\hline Chlamydia positivity in CSI-PCR n (\%) & $908(15.9)$ & & $908(54.0)$ & \\
\hline Gonorrhoea positivity n (\%) & $112(2.0)$ & $32(0.8)$ & $80(4.8)$ & $<0.001^{*}$ \\
\hline Migration background & & & & $<0.001^{*}$ \\
\hline Western & $4565(80.0)$ & $3382(84.1)$ & $1183(70.3)$ & \\
\hline Non-Western & $869(15.2)$ & $464(11.5)$ & $405(24.1)$ & \\
\hline Unknown & $270(4.7)$ & $176(4.4)$ & $94(5.6)$ & \\
\hline Educational level $\left.\right|^{\$ \#}$ & & & & $<0.001^{*}$ \\
\hline Low/Middle & $1170(20.5)$ & $661(16.5)$ & 509 (30.3) & \\
\hline High & $4529(79.5)$ & $3358(83.6)$ & $1171(69.7)$ & \\
\hline Age at sexual debut mean (SD) & $16.9(2.4)$ & $17.2(2.4)$ & $16.4(2.1)$ & $<0.001^{*}$ \\
\hline Lifetime sex partners & & & & $<0.001^{*}$ \\
\hline$<6$ & $1797(31.5)$ & $1.499(37.3)$ & $298(17.7)$ & \\
\hline $6-12$ & $2033(35.6)$ & $1424(35.4)$ & 609 (36.2) & \\
\hline$>12$ & $1874(32.9)$ & $1099(27.3)$ & $775(46.1)$ & \\
\hline Condom use with casual partners + & & & & $<0.001^{*}$ \\
\hline Never/not often & $340(6.0)$ & $243(6.1)$ & $97(5.8)$ & \\
\hline Sometimes & $1807(31.7)$ & $1100(27.4)$ & $707(42.1)$ & \\
\hline Always/mostly & $2579(45.9)$ & $1890(47.1)$ & $720(42.9)$ & \\
\hline No casual partners & $936(16.4)$ & $780(19.4)$ & $156(9.3)$ & \\
\hline Use of IUD & & & & 0.230 \\
\hline Never & $3672(64.4)$ & $2609(64.9)$ & $1063(63.2)$ & \\
\hline At least once & $2032(35.6)$ & $1413(35.1)$ & $619(36.8)$ & \\
\hline Smoking & & & & $<0.001^{*}$ \\
\hline Never & $2264(39.7)$ & $1724(42.9)$ & $540(32.1)$ & \\
\hline Sometimes/in the past & $2843(49.8)$ & $1960(48.7)$ & $883(52.5)$ & \\
\hline Daily & $597(10.5)$ & $338(8.4)$ & $259(15.4)$ & \\
\hline Number of chlamydia tests & & & & $<0.001^{*}$ \\
\hline 1 & $1042(18.3)$ & $931(23.2)$ & $111(6.6)$ & \\
\hline 2 & $1539(27.0)$ & $1222(30.4)$ & $317(18.9)$ & \\
\hline 3 & $1485(26.0)$ & $1032(25.7)$ & $453(26.9)$ & \\
\hline$>3$ & $1638(28.7)$ & $837(20.8)$ & $801(47.6)$ & \\
\hline \multicolumn{5}{|l|}{ Chlamydia symptoms ${ }^{\Omega}$} \\
\hline No & & - & $952(56.6)$ & \\
\hline Yes & & - & $730(43.4)$ & \\
\hline \multicolumn{5}{|l|}{ Number of chlamydia infections ${ }^{\ddagger}$} \\
\hline 1 & & - & $1325(78.8)$ & \\
\hline 2 & & - & $291(17.3)$ & \\
\hline 3 or more & & - & $66(3.9)$ & \\
\hline \multicolumn{5}{|l|}{ Age at first chlamydia infection } \\
\hline$<20$ & & - & $305(20.8)$ & \\
\hline $20-24$ & & - & $640(43.7)$ & \\
\hline$>24$ & & - & $519(35.5)$ & \\
\hline \multicolumn{5}{|l|}{ Chlamydia IgG antibodies (serology) } \\
\hline No & $3101(54.4)$ & $2572(64.0)$ & $529(31.5)$ & \\
\hline Yes & $569(10.0)$ & - & $569(33.8)$ & \\
\hline Unknown & $2034(35.7)$ & $1450(36.0)$ & $584(34.7)$ & \\
\hline
\end{tabular}

Chlamydia positive was defined as a positive PCR-test outcome in the CSI study (CSI-PCR), and/or the presence of chlamydia IgG and/or a self-reported chlamydia infection. \#At start of NECCST and based on 5699 observations, 5 missing. \$Educational level: Low/medium level of education: no education, primary education only, lower general secondary education and vocational education; high level of education: all other education levels. Chlamydia symptoms were vaginal discharge, abdominal pain or pain during intercourse and/or intermittent vaginal bleeding. + Based on 5693 observations, 11 missing. $\Omega$ based on 1665 observations, 15 missing values. $\ddagger$ based on 1639 observations, 43 missing. SD = standard deviation. $\mathrm{CSI}=$ Chlamydia Screening Implementation. $\mathrm{PCR}=$ polymerase chain reaction. IUD = Intrauterine Device. $\lg \mathrm{G}=$ Immunoglobulin G. * = statistically significant, $\mathrm{p}<0.05$. 
PID

Women with an episode of PID before exposure time were excluded $(n=6)$. In total, $159(2.8 \%$, 95\% Cl 2.4-3.2) women reported at least one episode of PID, of which 57 (35.9\%) were admitted to hospital. Of 33 (20.8\%) women with $>1$ reported PID episode, $45.5 \%$ were admitted to hospital at least once. The incidence rate of PID was 1.8 per 1000py (95\% Cl 1.6-2.2). Chlamydia positives had a higher incidence rate of 4.4 episodes of PID per 1000py $(95 \% \mathrm{Cl} 3.3-5.7)$ compared to 1.4 per 1000py (95\% Cl 1.1-1.7) for chlamydia negatives. Incidence rates among the different chlamydia parameters, CSI-PCR +, chlamydia antibody presence or self-reported infections, were $4.5(95 \% \mathrm{Cl} 2.6-7.9), 5.2(95 \% \mathrm{Cl} 2.9-9.1)$ and $4.1(95 \% \mathrm{Cl} 2.8-5.8)$ per 1000py, respectively. In multivariable analysis, adjusted for age and educational level, chlamydia positivity remained strongly associated with PID (adjusted HR 2.22, 95\%Cl 1.57-3.13), Table 2 and Figure 1.

Table 2. Association between chlamydia positivity and pelvic inflammatory disease, ectopic pregnancy, and tubal factor infertility in women participating in NECCST.

\begin{tabular}{|c|c|c|c|c|c|c|c|c|}
\hline & \multirow{2}{*}{$\begin{array}{l}\text { Cases } \\
\mathrm{N}^{*}\end{array}$} & \multirow{2}{*}{$\begin{array}{l}\text { Time } \\
\text { Person-years }{ }^{\#}\end{array}$} & \multicolumn{3}{|c|}{ Crude hazard ratio } & \multicolumn{3}{|c|}{ Adjusted Hazard Ratio ${ }^{a, b, c}$} \\
\hline & & & $\mathrm{HR}$ & $95 \% \mathrm{Cl}$ & $P$ value & $\mathrm{aHR}$ & $95 \% \mathrm{Cl}$ & $P$ value \\
\hline \multicolumn{9}{|c|}{ Pelvic Inflammatory Disease } \\
\hline Chlamydia negative & 100 & 71,029 & 1 & & & 1 & & \\
\hline Chlamydia positive & 59 & 14,320 & 2.60 & $1.85-3.66$ & $<0.0001$ & 2.22 & $1.57-3.13$ & $<0.0001$ \\
\hline \multicolumn{9}{|l|}{ Ectopic pregnancy } \\
\hline Chlamydia negative & 41 & 71,412 & 1 & & & 1 & & \\
\hline Chlamydia positive & 11 & 14,684 & 0.99 & $0.49-1.97$ & 0.9720 & 0.80 & $0.39-1.63$ & 0.5335 \\
\hline \multicolumn{9}{|l|}{ Tubal factor infertility } \\
\hline Chlamydia negative & 15 & 71,523 & 1 & & & 1 & & \\
\hline Chlamydia positive & 18 & 14,699 & 4.27 & $2.08-8.77$ & 0.0001 & 4.22 & $2.05-8.69$ & 0.0001 \\
\hline
\end{tabular}

Chlamydia positive was defined as a positive PCR-test outcome in the CSI study (CSI-PCR), and/or the presence of chlamydia IgG and/or a self-reported chlamydia infection. For these analyses, multiple imputations were used to estimate time of first chlamydia infection in women without a known first year of chlamydia infection. *Median cases of 15 multiple imputation datasets. "Estimated from 15 multiple imputation datasets. a PID Model adjusted for age (time-varying) and educational level. ${ }^{b}$ Ectopic pregnancy model adjusted for age (time-varying), educational level, number of lifetime partners and migration background. ${ }^{\mathrm{C}} \mathrm{TFI}$ model adjusted for age (time-varying). $\mathrm{HR}=$ Hazard Ratio. $\mathrm{Cl}=\mathrm{Confidence} \mathrm{Interval.}$

\section{Ectopic pregnancy}

In total, 2633 (46.2\%) women were pregnant at least once or tried to become pregnant. 52 (0.9\%, $95 \% \mathrm{Cl}$ 0.7-1.2) women reported one or more ectopic pregnancies. The incidence rate of ectopic pregnancy was 0.6 per 1000py $(95 \% \mathrm{Cl} 0.5-0.8)$. Ectopic pregnancy rates were not different between chlamydia positives $(0.8$ per $1000 \mathrm{py}, 95 \% \mathrm{Cl} 0.4-1.5)$ and chlamydia negatives $(0.6$ per 1000py, 95\% $\mathrm{Cl} 0.4-0.8)$, nor in multivariable analysis after adjustment for age, educational level, number of lifetime partners and migration background (aHR 0.80, 95\% Cl 0.39-1.63), Table 2, Figure 1. Incidence rates among the different chlamydia parameters, CSI-PCR+, chlamydia antibody presence or self-reported infections, were $0.4(95 \% \mathrm{Cl} 0.1-2.6), 0.4(95 \% \mathrm{Cl} 0.1-3.0)$ and $1.1(95 \% \mathrm{Cl}$ 0.5-2.1) per 1000py, respectively. 


\section{Tubal factor infertility}

In total, $33(0.6 \%, 95 \% \mathrm{Cl} 0.4-0.8)$ women reported to be diagnosed with TFI. The incidence rate was 0.4 per 1000 person-years $(95 \% \mathrm{Cl}$ 0.3-0.5). Among chlamydia positives, the incidence rate of TFI was considerably higher compared to chlamydia negatives: 1.3 per $1000 \mathrm{py}(95 \% \mathrm{Cl}$ 0.8-2.1) and 0.2 per 1000py $(95 \% \mathrm{Cl} 0.1-0.4)$ respectively. Incidence rates among the different chlamydia parameters, CSI-PCR +, Chlamydia antibody presence or self-reported infections, were 1.1 (95\% Cl 0.4-3.4), 0.8 (95\%Cl 0.2-3.4) and 1.4 (95\% Cl 0.8-2.6) per 1000py, respectively. Chlamydia positivity was highly associated with TFI: aHR 4.22, $(95 \% \mathrm{Cl} 2.05-8.69)$, Table 2 and Figure 1. The model was adjusted for age.

Sensitivity analyses on all three outcomes (PID, ectopic pregnancy, TFI) using data restrictions as indicated in methodology section yielded mostly similar results, Figure 2.

Risk factors for long-term sequelae among chlamydia-positive women

In multivariable analyses, having had chlamydia symptoms and IUD insertion were associated with PID, Supplementary Table S1. For TFI, a younger age at first chlamydia infection ( $<20$ years) was the only risk factor in univariable analyses. For both PID and TFI, we were unable to determine an increased risk for women with an unnoticed chlamydia infection (chlamydia IgG-positive only) compared to women with a known and presumably treated infection.

Associations between risk factors and outcomes regardless of chlamydia-status can be found in Supplementary Table S2. 
A

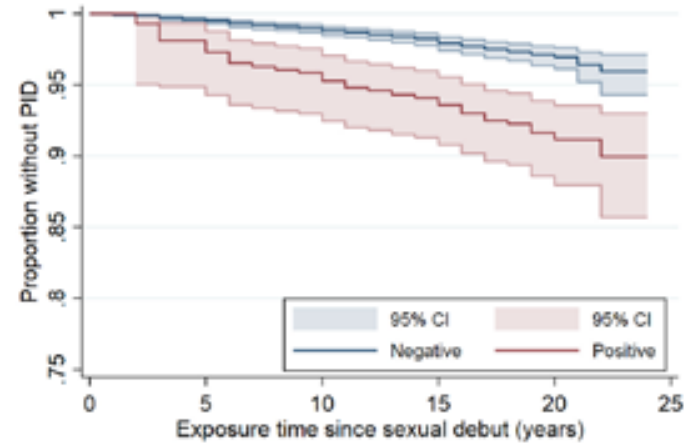

Number at risk

$\begin{array}{ccccccc}\text { Chlamydia negative } & 5433 & 4970 & 3981 & 2217 & 596 & 0 \\ \text { Chlamydia positive } & 52 & 486 & 998 & 769 & 196 & 0\end{array}$

B

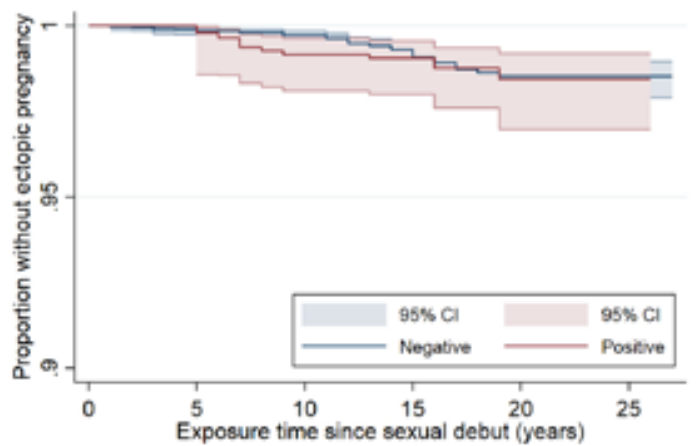

Number at risk

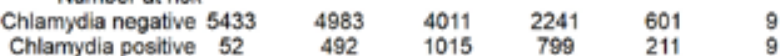

C

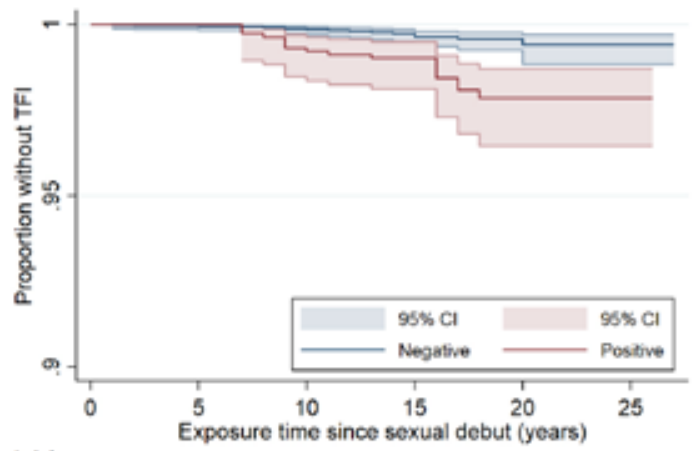

Number at risk

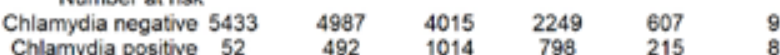

Figure 1. Kaplan-Meier plots of time (years) by chlamydia status since sexual debut. A. Pelvic inflammatory disease. B. Ectopic pregnancy. C. Tubal factor infertility. Chlamydia positive was defined as a positive PCR-test outcome in the CSI study (CSI-PCR), and/or the presence of chlamydia IgG and/or a self-reported chlamydia infection. 


\section{Forest plot sensitivity analyses}

HR (902) a)

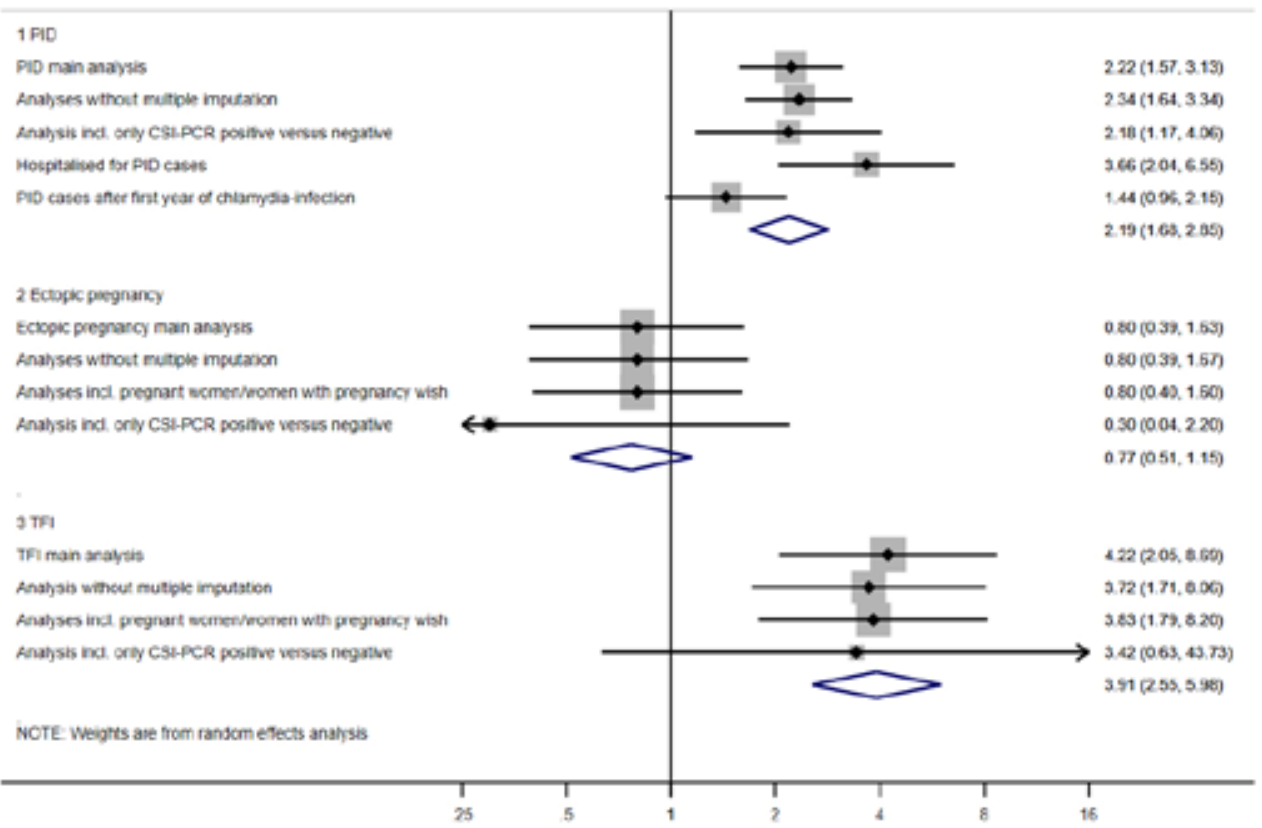

Figure 2. Forest plot of various sensitivity analyses of the risk for PID, ectopic pregnancy and TFI between chlamydia positive and chlamydia negative women in hazard ratio's and $95 \% \mathrm{Cl}$. $\mathrm{HR}=$ hazard ratio. $\mathrm{Cl}=$ confidence interval.

\section{Discussion}

We estimated risks of PID, ectopic pregnancy, and TFI in a unique cohort of over 5,500 women with up to eight years of follow-up. Chlamydia positivity was strongly associated with PID, as shown by a twofold higher risk, and with TFI, by a fourfold higher risk. Incidence rates and risks on sequelae were comparable among all chlamydia positives (either PCR positive, antibody positive or self-reported). Among chlamydia-positive women, PID was found to be more common in women who had symptoms during infection. For TFI among chlamydia-positive women, young age $(<20)$ at first chlamydia infection was the strongest risk factor. We did not find an association between chlamydia positivity and ectopic pregnancy. Although differences in risk between chlamydiapositive and chlamydia-negative women were considerable and highly significant, the cumulative incidence for any of the sequelae studied was low $(<5 \%)$.

This cohort study yielded an exhaustive dataset in which chlamydia-related sequelae were determined. First, over 5,500 women were followed since 2008 with multiple data collection 
points. Second, we adopted a thorough approach in determining previous chlamydia infections using three outcome measures: CSI-PCR, serology and self-reporting. Thereby increasing the true proportion of chlamydia-positive women, however we cannot completely exclude infections in the negative group due to moderate sensitivity of the chlamydia IgG ELISA test. Third, we were able to adjust the relation between chlamydia and its sequelae for multiple confounders, i.e. sexual risk behaviour, previous pregnancies and pregnancy wishes, demographics and lifestyle factors. Therefore, our results may approach true risk estimates by reducing some important uncertainties(5).

This study had several limitations. First, we used self-reported data on PID, ectopic pregnancy and TFI and in part on chlamydia infections, which are subject to recall bias. In addition, PID diagnoses are imprecise since a non-invasive gold standard test is not available(25). Both over-reporting and diagnostic bias towards chlamydia-positive women could have taken place(5), possibly leading to an overestimation of the risk among chlamydia-positive women. TFI was based on self-reports, but partly validated in medical registers, showing good agreement. Second, because the NECCST cohort is based on a sample of the CSI study, we have a non-representative sample which decreased generalizability of the results. Third, timing of events was defined in years. As PID sometimes occurred in the same year as the first chlamydia infection, it cannot be ruled out that some PID cases might have occurred before the first chlamydia infection. Sensitivity analyses excluding PID cases that occurred in the same year as the chlamydia infection still yielded an increased risk for chlamydia-positive women, though not significant. Thus, while the largest risk for PID following a chlamydia infection was in the year of infection, the majority of PIDs occurred thereafter. Lastly, to draw conclusions on a possible association between chlamydia and ectopic pregnancy, which remains inconclusive also in previous studies $(9,26)$, longer follow-up of NECCST will be needed(20).

Incidence rates of PID and TFI among chlamydia-positive women found in our study (4.4/1000 and 1.1/1000py, respectively) were comparable with results from two Danish studies and an Australian population-based cohort study. However, our incidence rate for ectopic pregnancy was lower: 0.8/1000py. These studies found incidence rates of 4.0-8.0/1000py for PID, 2.8/1000py for ectopic pregnancy and 0.7/1000py for TFI and reported cumulative incidences of 2-3\%, 2-3\% and 0.6\%, respectively $(9,10,27)$. For PID and TFI, the Danish and Australian studies found smaller differences in risk between chlamydia positives and negatives than we did: for PID 1.3-1.8 and for TFI 1.3(9, $10,27)$ compared to our HRs of 2.2 and 4.2, respectively. A different comparison was made in these studies because their definition of chlamydia-negative was based on negative test (NAAT) results, excluding non-tested women, who were at lower risk than women who tested negative. We want to be able to identify chlamydia-positive women with the highest risk of complications. Identified risk factors can help develop chlamydia specific prevention methods. In chlamydianegative women, it is more likely that the cause of complications is due to causes other than chlamydia. The strongest risk factors for PID among chlamydia-positive women were chlamydia symptoms and insertion of an IUD. However, women with an IUD might be more focused on abdominal pain and visit a doctor earlier compared to women without an IUD (diagnostic bias) (28). Thereby, only part of PID, but also ectopic pregnancy and TFI, is caused by chlamydia, 
estimated population excess fractions are between 20-30\% for PID, around 5\% for ectopic pregnancy and $30 \%$ for $\operatorname{TFI}(7)$.

This study adds to the evidence that chlamydia increases the risk for PID and TFI in women even if the infection was treated(29), but also showed that incidence rates were small. However, alertness for PID in years after infection remains warranted among health care providers, especially in women who experienced chlamydia-related symptoms during infection. Though chlamydia is an important causative agent for TFI (i.e. fourfold increased risk) during the eight-year follow-up time of our cohort, TFI was only found in $1 \%$ of women following chlamydia infection, in accordance with modelling studies(12). It is important to identify that $1 \%$ of women with high risk. The following step in NECCST is to assess single nucleotide polymorphisms (SNPS) associations.

The present results underscore the need for effective prevention of reproductive tract complications following chlamydia infection. Given the small cumulative risks and the lack of evidence that chlamydia screening is effective in decreasing prevalence, it might be more effective to focus on women at highest risk for complications rather than promoting widespread chlamydia screening(30). 


\section{References}

1. Newman L, Rowley J, Vander Hoorn S, Wijesooriya NS, Unemo M, Low N, et al. Global Estimates of the Prevalence and Incidence of Four Curable Sexually Transmitted Infections in 9 2012 Based on Systematic Review and Global Reporting. PLoS One. 2015;10(12):e0143304.

2. Woodhall SC, Wills GS, Horner PJ, Craig R, Mindell JS, Murphy G, et al. Chlamydia trachomatis Pgp3 Antibody Population Seroprevalence before and during an Era of Widespread Opportunistic Chlamydia Screening in England (1994-2012). PLoS One. 2017;12(1):e0152810.

3. van Aar F, de Moraes M, Morre SA, van Bergen $\mathrm{JE}$, van der Klis FR, Land JA, et al. Chlamydia trachomatis IgG seroprevalence in the general population of the Netherlands in 1996 and in 2007: differential changes by gender and age. Sex Transm Infect. 2014;90(5):434-40.

4. Low N, McCarthy A, Macleod J, Salisbury C Campbell R, Roberts TE, et al. Epidemiological, social, diagnostic and economic evaluation of population screening for genital chlamydial infection. Health Technol Assess. 2007;11(8):iiiiv, ix-xii, 1-165.

5. Haggerty CL, Gottlieb SL, Taylor BD, Low N, Xu F, Ness RB. Risk of sequelae after Chlamydia trachomatis genital infection in women. J Infect Dis. 2010;201 Suppl 2:S134-55.

6. Land J, Van Bergen J, Morre S, Postma M. Epidemiology of Chlamydia trachomatis infection in women and the cost-effectiveness of screening. Human reproduction update. 2009:dmp035.

7. Price $\mathrm{MJ}$, Ades $\mathrm{AE}$, Soldan $\mathrm{K}$, Welton $\mathrm{NJ}$, Macleod J, Simms I, et al. The natural history of Chlamydia trachomatis infection in women: a multi-parameter evidence synthesis. Health Technol Assess. 2016;20(22):1-250.

8. van Valkengoed IG, Morré $S A$, van den Brule AJ, Meijer CJ, Bouter LM, Boeke AJP. Overestimation of complication rates in evaluations of Chlamydia trachomatis screening programmes-implications for costeffectiveness analyses. International Journal of Epidemiology. 2004;33(2):416-25.

9. Davies B, Turner KM, Frolund M, Ward H, May MT, Rasmussen S, et al. Risk of reproductive complications following chlamydia testing: a population-based retrospective cohort study in Denmark. Lancet Infect Dis. 2016.

10. Low N, Egger $M$, Sterne JA, Harbord RM, Ibrahim F, Lindblom B, et al. Incidence of severe reproductive tract complications associated with diagnosed genital chlamydial infection: the Uppsala Women's Cohort Study. Sex Transm Infect. 2006;82(3):212-8.

11. Bakken IJ, Skjeldestad FE, Lydersen S, Nordbo SA. Births and ectopic pregnancies in a large cohort of women tested for Chlamydia trachomatis. Sex Transm Dis. 2007;34(10):73943.

12. Kavanagh $\mathrm{K}$, Wallace LA, Robertson C, Wilson $\mathrm{P}, \mathrm{Scoular}$ A. Estimation of the risk of tubal factor infertility associated with genital chlamydial infection in women: a statistical modelling study. International journal of epidemiology. 2013;42(2):493-503.

13. Oakeshott P, Kerry S, Aghaizu A, Atherton H, Hay S, Taylor-Robinson D, et al. Randomised controlled trial of screening for Chlamydia trachomatis to prevent pelvic inflammatory disease: the POPI (prevention of pelvic infection) trial. BMJ. 2010;340:c1642.

14. van den Broek IV, van Bergen JE, Brouwers EE, Fennema JS, Gotz HM, Hoebe CJ, et al. Effectiveness of yearly, register based screening for chlamydia in the Netherlands: controlled trial with randomised stepped wedge implementation. BMJ. 2012;345:e4316.

15. Low N, Redmond S, Uuskula A, van Bergen J, Ward $\mathrm{H}$, Andersen $\mathrm{B}$, et al. Screening for genital chlamydia infection. Cochrane Database Syst Rev. 2016;9:CD010866.

16. Menon S, Timms P, Allan JA, Alexander K, Rombauts $L$, Horner $P$, et al. Human and 
Pathogen Factors Associated with Chlamydia 23 trachomatis-Related Infertility in Women. Clin Microbiol Rev. 2015;28(4):969-85.

17. Tiitinen A, Surcel $\mathrm{H}-\mathrm{M}$, Halttunen $\mathrm{M}$, Birkelund S, Bloigu A, Christiansen $G$, et al. Chlamydia trachomatis and chlamydial heat shock protein 60-specific antibody and cell-mediated responses predict tubal factor infertility. Human Reproduction. 2006;21(6):1533-8.

18. Morre SA, Karimi O, Ouburg S. Chlamydia trachomatis: identification of susceptibility markers for ocular and sexually transmitted infection by immunogenetics. FEMS Immunol Med Microbiol. 2009;55(2):140-53.

19. Hay PE, Kerry SR, Normansell R, Horner PJ, Reid F, Kerry SM, et al. Which sexually active young female students are most at risk of pelvic inflammatory disease? A prospective study. Sex Transm Infect. 2016;92(1):63-6.

20. Hoenderboom BM, van Oeffelen AA, van Benthem BH, van Bergen JE, Dukers-Muijrers $\mathrm{NH}$, Gotz HM, et al. The Netherlands Chlamydia cohort study (NECCST) protocol to assess the risk of late complications following Chlamydia trachomatis infection in women. BMC Infect Dis. 2017; 17(1):264

21. Hoenderboom BM, van Ess EF, van den Broek IVF, van Loo IHM, Hoebe C, Ouburg S, et al. Chlamydia trachomatis antibody detection in home-collected blood samples for use in epidemiological studies. J Microbiol Methods. 2017;144:164-7.

22. Morre SA, Munk C, Persson K, Kruger-Kjaer S, van Dijk R, Meijer CJLM, et al. Comparison of Three Commercially Available Peptide-Based Immunoglobulin $\mathrm{G}$ ( $\mathrm{Ig} \mathrm{G})$ and IgA Assays to Microimmunofluorescence Assay for Detection of Chlamydia trachomatis Antibodies. Journal of Clinical Microbiology. 2002;40(2):584-7.
23. Raghunathan TE, Lepkowski, J.M., Van Hoewyk, J., Solenberger, P.,. A Multivariate Technique for Multiply Imputing Missing Values Using a Sequence of Regression Models. Survey Methodology. 2001;27(No. 1):85-95.

24. de Boer EJ, den Tonkelaar I, Burger CW, van Leeuwen FE, Group OP. Validity of selfreported causes of subfertility. Am J Epidemiol. 2005;161(10):978-86.

25. Simms I, Warburton F, Westrom L. Diagnosis of pelvic inflammatory disease: time for a rethink. Sex Transm Infect. 2003;79(6):491-4.

26. Andersen B, stergaard L, Puho E, Skriver MV, Sch??nheyder HC. Ectopic Pregnancies and Reproductive Capacity After Chlamydia trachomatis Positive and Negative Test Results: A Historical Follow-Up Study. Sexually Transmitted Diseases. 2005;32(6):377-81.

27. Reekie J, Donovan B, Guy R, Hocking JS, Kaldor JM, Mak DB, et al. Risk of Pelvic Inflammatory Disease in Relation to Chlamydia and Gonorrhea Testing, Repeat Testing, and Positivity: A Population-Based Cohort Study. Clin Infect Dis. 2017.

28. Hubacher D, Grimes DA, Gemzell-Danielsson $K$. Pitfalls of research linking the intrauterine device to pelvic inflammatory disease. Obstet Gynecol. 2013;121(5):1091-8.

29. Dahlberg J, Hadad R, Elfving K, Larsson I, Isaksson J, Magnuson A, et al. Ten years transmission of the new variant of Chlamydia trachomatis in Sweden: prevalence of infections and associated complications. Sex Transm Infect. 2017.

30. Unemo M, Bradshaw CS, Hocking JS, de Vries HJC, Francis SC, Mabey D, et al. Sexually transmitted infections: challenges ahead. Lancet Infect Dis. 2017;17(8):e235-e79. 


\section{Supplementary material}

- $\quad$ Figure S1. Study flowchart.

- $\quad$ Figure S2. Venn diagram of chlamydia positivity.

- Table S1. Risk factors for pelvic inflammatory disease and tubal factor infertility among chlamydia-positive women.

- $\quad$ Table S2. Risk factors for pelvic inflammatory disease and tubal factor infertility 


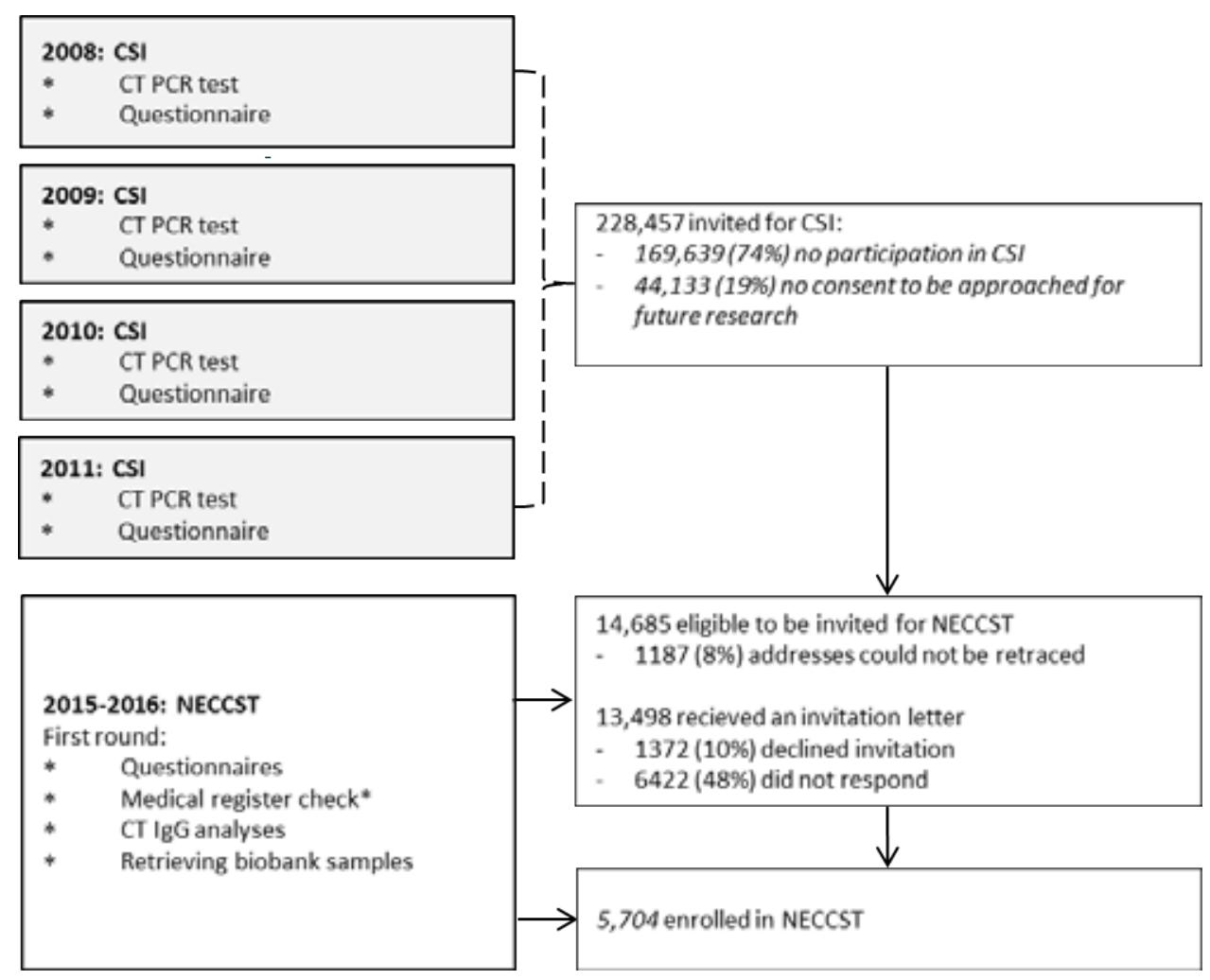

Figure S1. Study flowchart (20). CT = Chlamydia trachomatis. PCR $=$ polymerase chain reaction. CSI $=$ Chlamydia Screening Implementation. IgG = Immunoglobulin G. NECCST = Netherlands Chlamydia Cohort Study. 


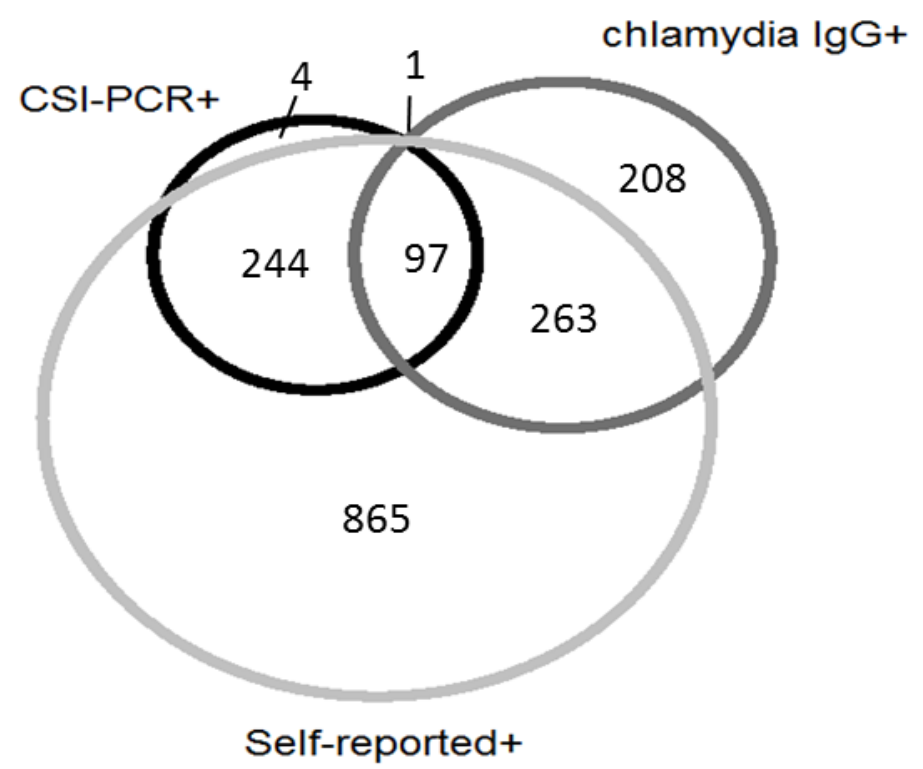

Figure S2. Venn diagram of chlamydia positivity. Numbers are based on 1682 chlamydia-positive women with self-reported infection, positive CSI-PCR and/or chlamydia IgG antibodies. Chlamydia IgG = chlamydia Immunoglobulin G. CSI = Chlamydia Screening Implementation. 


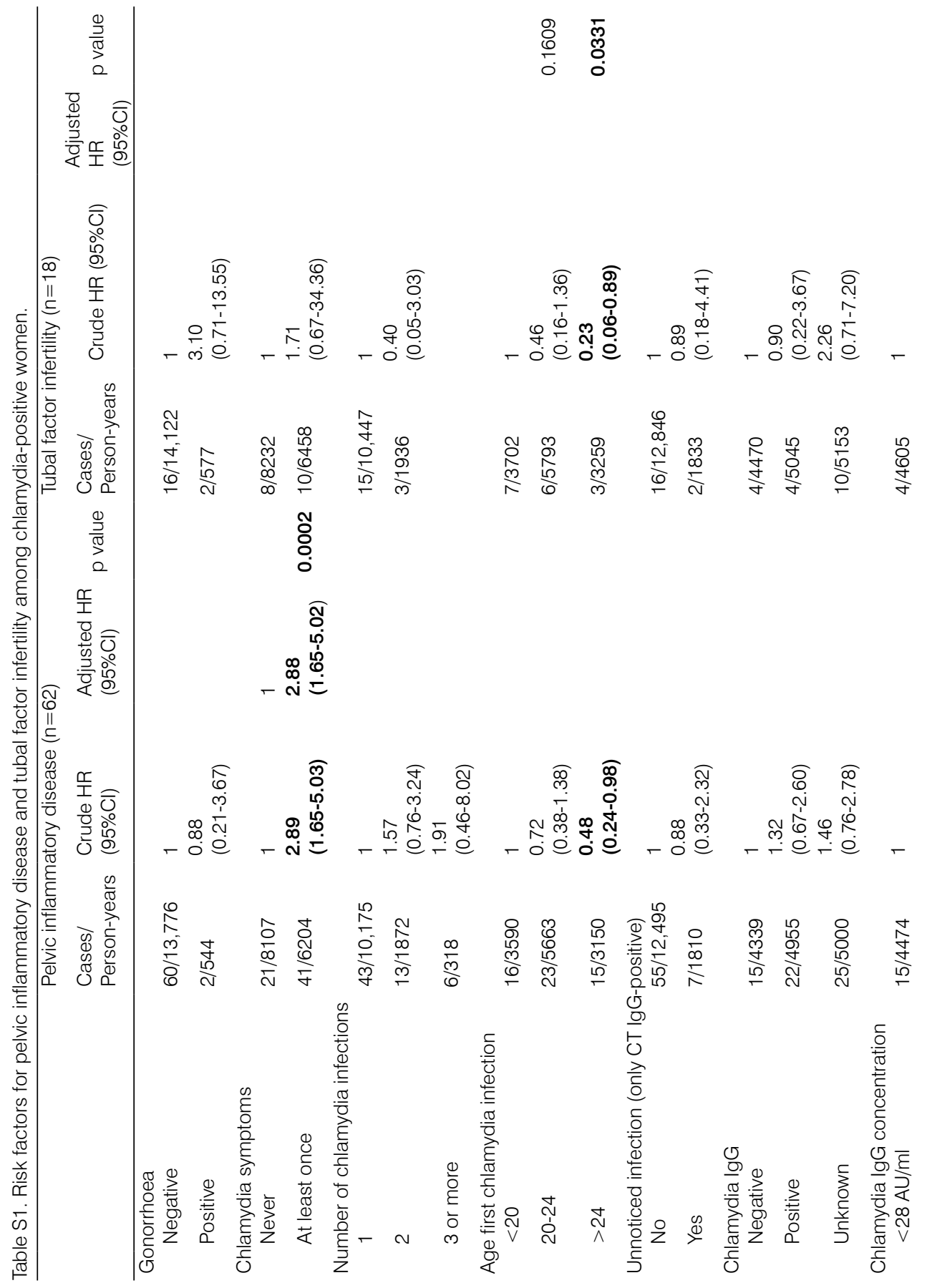




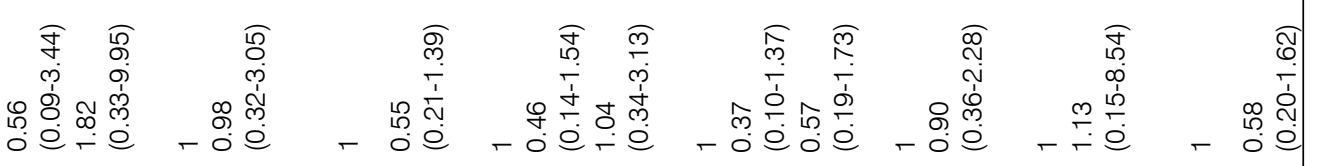

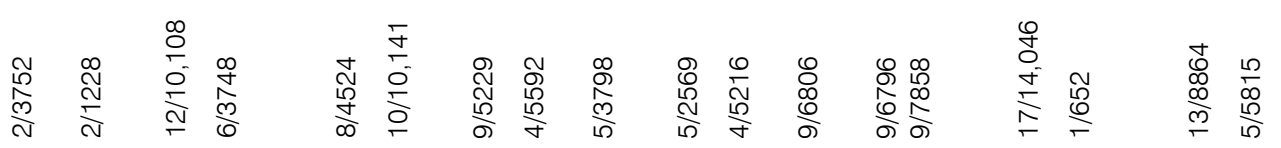

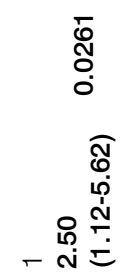

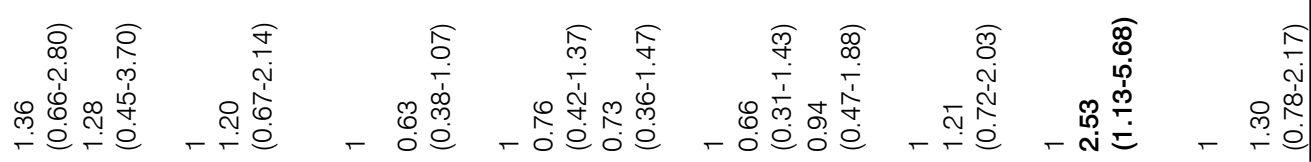

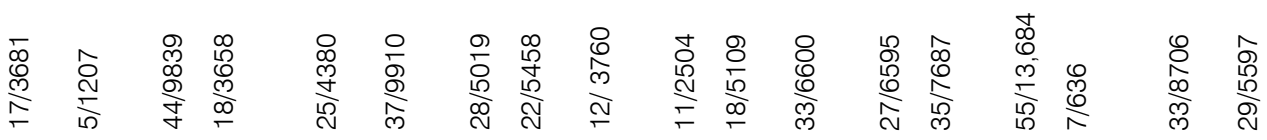

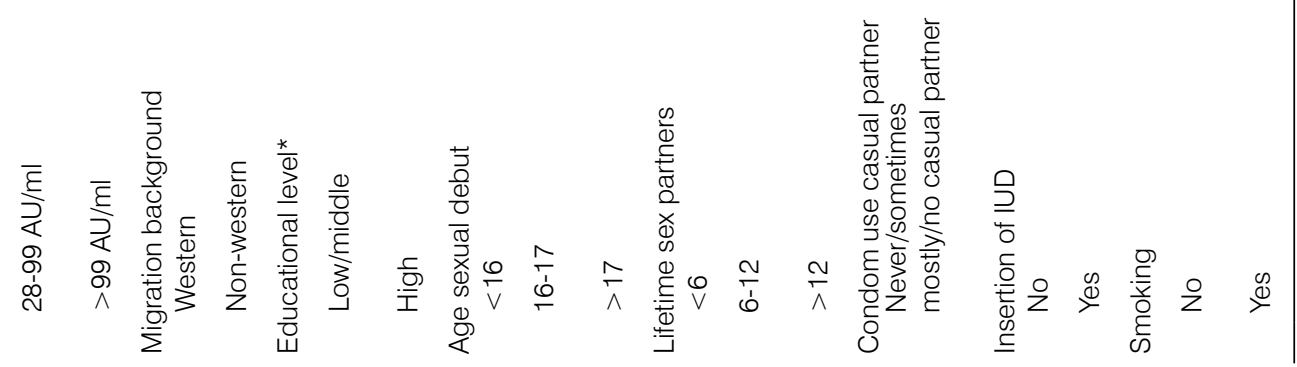




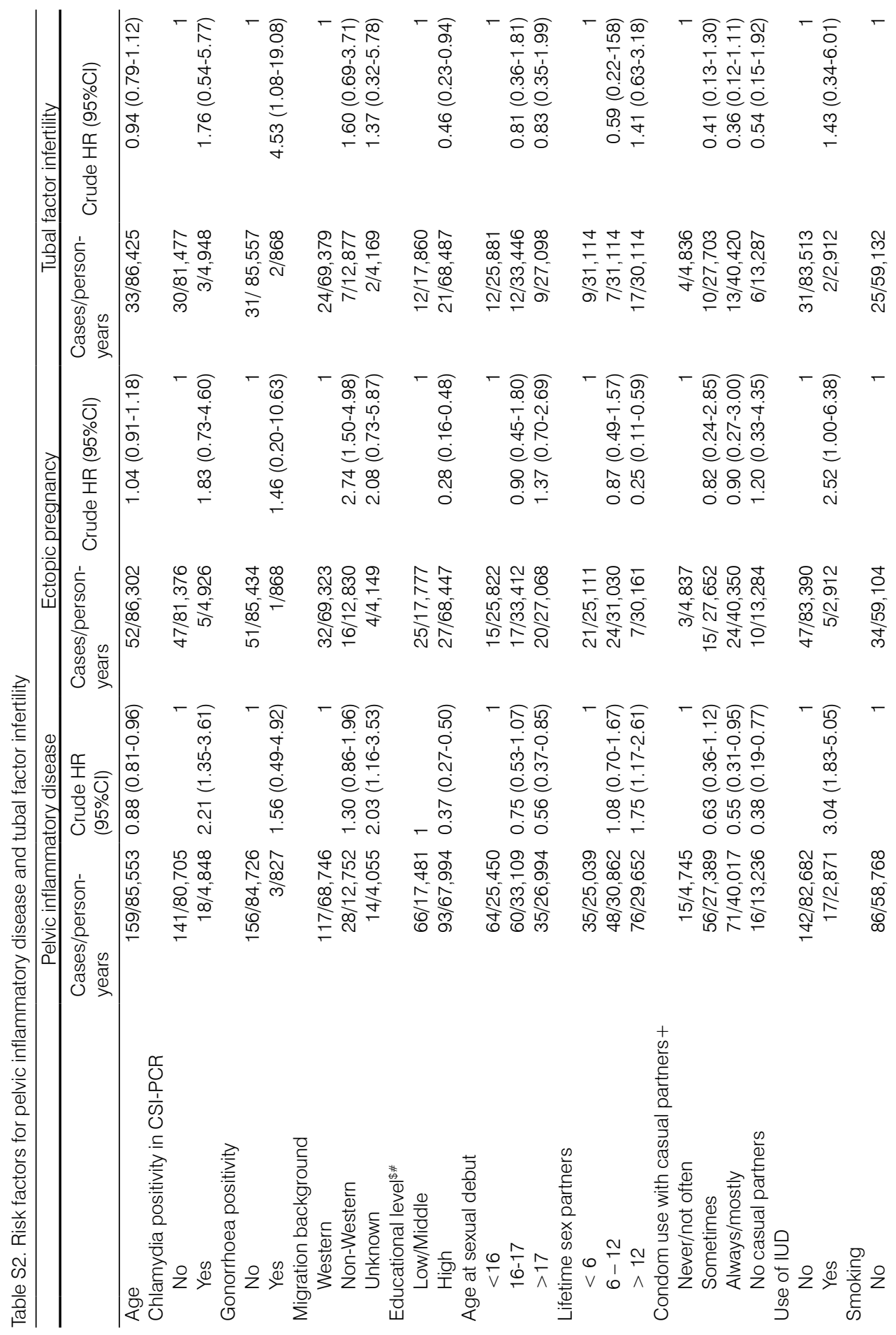




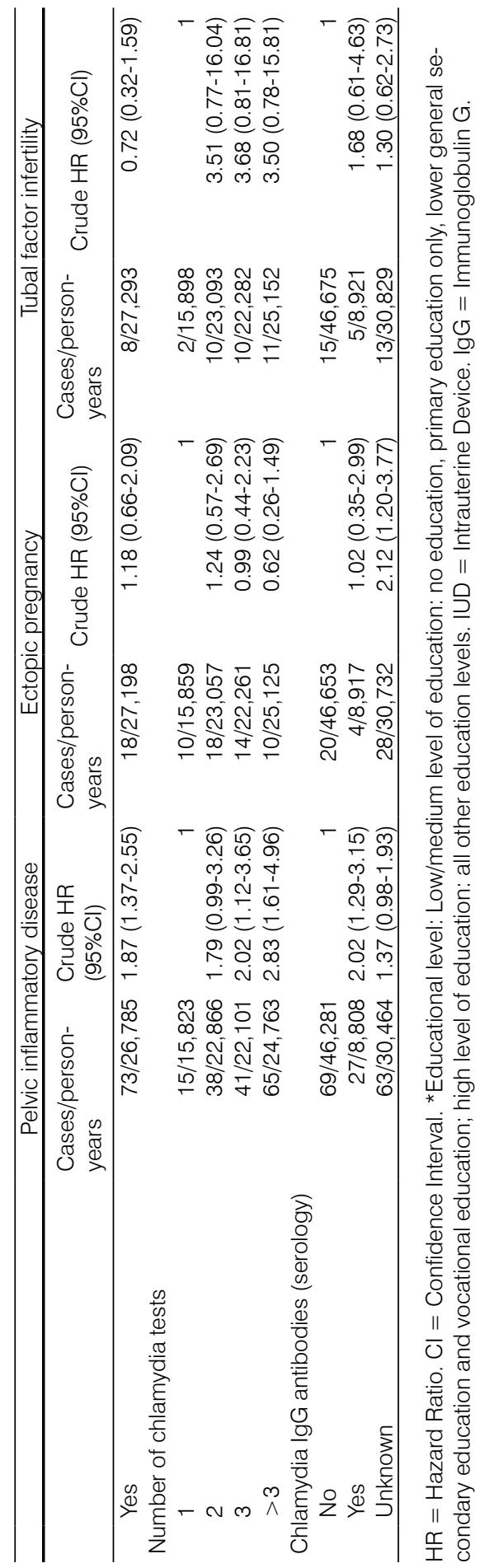





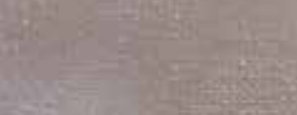

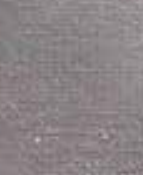

\section{sisting}

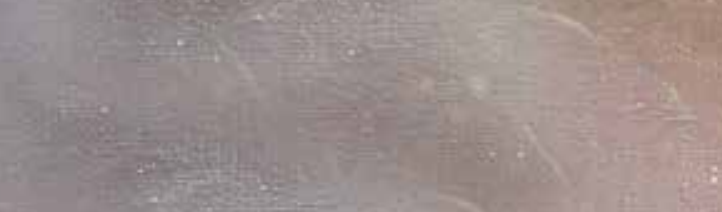

जince

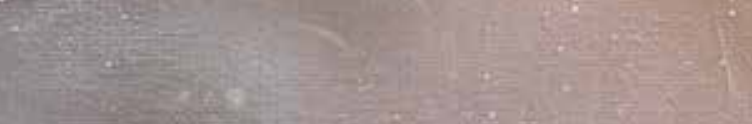

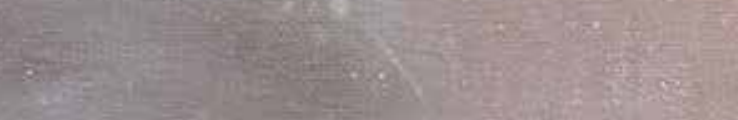

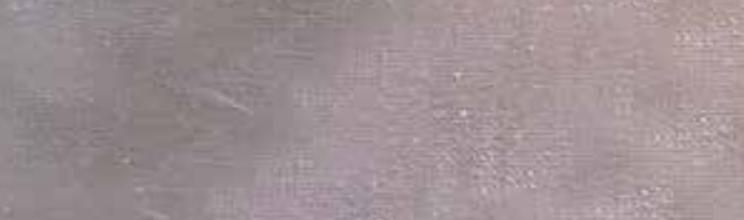

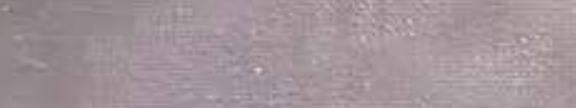

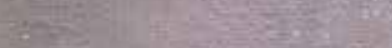

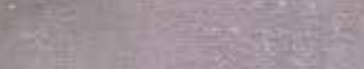

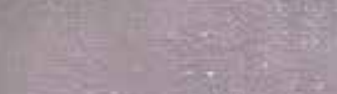

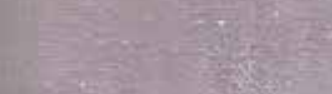

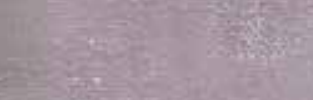

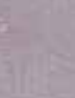

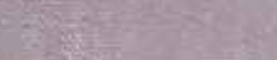

netwo

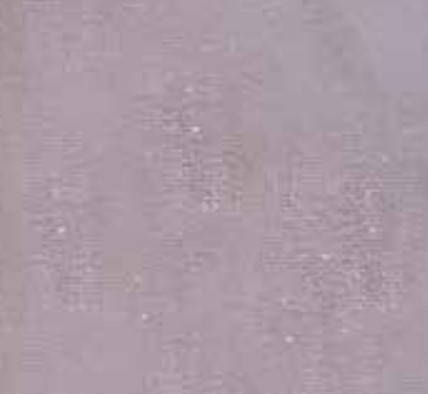

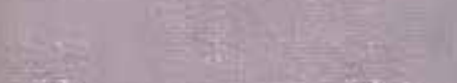

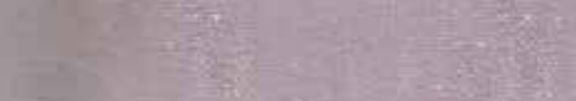




\section{8}

\section{Pregnancies and time to pregnancy in women with and without a previous Chlamydia trachomatis infection}

Bernice M Hoenderboom, Jan EAM van Bergen, Nicole HTM Dukers-Muijrers, Hannelore M Götz, Christian JPA Hoebe, Henry JC de Vries, Ingrid VF van den Broek, Frank de Vries, Jolande A Land, Marianne AB van der Sande, Servaas A Morré, Birgit $\mathrm{HB}$ van Benthem 


\section{Abstract}

Background: A Chlamydia trachomatis infection (chlamydia) can result in tubal factor infertility in women. To assess if this association results in fewer pregnant women, we aimed to assess pregnancy incidences and time to pregnancy among women with a previous chlamydia infection compared to women without one and who were participating in the Netherlands Chlamydia Cohort Study (NECCST).

Methods: NECCST is a cohort of women of reproductive age tested for chlamydia in a chlamydia screening trial (CSI) between 2008-2011 and re-invited for NECCST in 2015-2016. Chlamydia status (positive/negative) was defined using CSI-NAAT results, chlamydia IgG presence in serum or self-reported chlamydia infections. Data on pregnancies was collected via questionnaires in 20152016 and 2017-2018. Overall pregnancies (i.e. planned and unplanned) and time to pregnancy (among women with a pregnancy intention) were compared between chlamydia-positive and chlamydia-negative women using Cox regressions.

Results: Of 5,704 women enrolled, 1717 (30.1\%, 95\%Cl 28.9-31.3) women was chlamydia positive. Overall pregnancy proportions were similar in chlamydia-positive and -negative women $(49.0 \%$, $95 \% \mathrm{Cl} 46.5-51.4$ versus 50.5\%, 95\% Cl 48.9-52.0). Pregnancies per 1000 person-years was 53.2 (95\%Cl 51.5-55.0) for chlamydia negatives and 83.0 (95\% Cl 78.5-87.9) for chlamydia positives. Among women with a pregnancy intention, $12 \%$ of chlamydia-positive women had a time to pregnancy of $>12$ months compared to $8 \%$ of chlamydia negatives, $p<0.01$.

Conclusions: Overall pregnancy rates were not lower in chlamydia-positive women compared to chlamydia-negative women, but among women with a pregnancy intention time to pregnancy was longer and pregnancy rates were lower in chlamydia-positive women. 


\section{Introduction}

Studies over the last twenty years show a stable three percent Chlamydia trachomatis (chlamydia) infection prevalence among Dutch women in the ages between 16-34 years(1-3). Chlamydia can cause pelvic inflammatory disease (PID), which can then result in tubal scarring due to an intense and chronic inflammatory response(4). Tubal scarring may lead to tubal factor infertility (TFI)(5). In the Netherlands, TFI was diagnosed in approximately $11 \%$ of infertile couples between 20022004(6). It is estimated that about one percent of women will develop TFI following chlamydia infection $(7,8)$. In the case of high chlamydia prevalence in a population, this will result in considerable numbers of infertile women. Women with chlamydia-related TFI may need medical assistance, for example in vitro fertilization (IVF) to become pregnant(9). However, the mean chance of a live birth following IVF - with large variability because of determinants such as age and cause of infertility - has been estimated at only about $42 \%$ after three complete IVF cycles(10). Hence, we hypothesized that pregnancy rates are lower, or time to pregnancy is longer among women with a previous chlamydia infection versus women without a previous chlamydia infection. So far, only a few studies have assessed the association between chlamydia infections and subsequent pregnancies or births. A large retrospective study (>20,000 women included) compared birth rates between women who tested chlamydia-positive either by serology or DNA test compared to women with negative tests only(11). No differences in birth rates between chlamydia-positive and -negative women were found. Similar results were found in a historical follow-up study from Denmark in which again >20,000 women were included; birth rates (alive or stillborn) were similar between chlamydia-seropositive and -seronegative women(12). In both studies, exposure time was calculated from a first chlamydia test onwards. Factors such as sexual behavior, previous contraceptive use, intention to conceive, and time to pregnancy in months were unknown in these studies and noted as limitations. These limitations need to be addressed to determine true differences in pregnancies between chlamydia-positive and -negative women. One main aim of chlamydia control is to prevent complications like infertility through early testing and treating infections. It is therefore important to not only focus on chlamydial-induced infertility, but also on fertility after infection. We investigated differences in pregnancy incidences and time to pregnancy between women with and without a previous chlamydia infection, where time was defined as the months women attempted to conceive, and where factors such as sexual (risk) behavior, intention to conceive, and contraceptive use were included.

\section{Materials and Methods}

\section{Study design and participants}

In the Netherlands Chlamydia Cohort Study (NECCST), Dutch women of reproductive age are followed until 2022. The design of NECCST has been described previously(13). Briefly, in 20082011, women between the age of 16-29 participated in the population-based Chlamydia Screening Implementation study (CSI)(2). During CSI, women received annual invitations for chlamydia 
nucleic acid amplification tests (NAAT). Women who participated at least once and gave consent to be approached for further research were invited to participate in NECCST in 2015-2016, Figure I. Participants gave online informed consent to comply with study procedures for the collection of repeat questionnaires and biological samples.

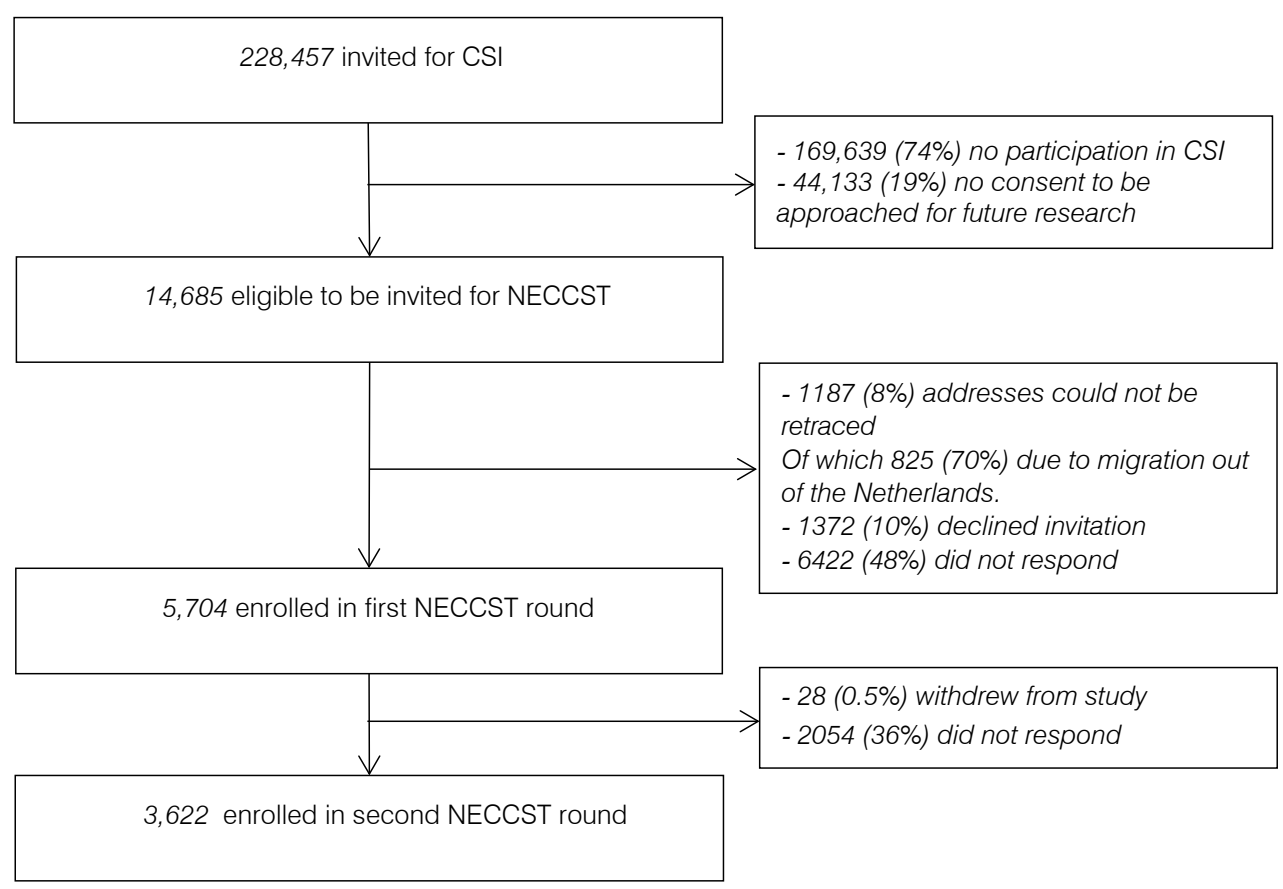

Figure 1. Study inclusion flowchart. CSI = chlamydia screening implementation study, NECCST = Netherlands Chlamydia Cohort Study(22).

\section{Procedures}

The first NECCST data collection round included an electronic questionnaire followed by a test kit for self-collection of blood via a finger-prick for chlamydia IgG analysis. The capillary blood samples, collected in tubes [BD Microtainer SST, USA] were returned to the laboratory via mail. Time between blood collection and storage was a median of four days (IQR 3-5), which was previously validated(14). Serum samples were stored at $-20^{\circ} \mathrm{C}$ until thawed for ELISA assay (Medac CT IgG ELISA plus, Wedel, Germany) with a sensitivity of $71 \%$ and specificity of $97 \%(14,15)$. Presence of chlamydia IgG served as a marker for previous infection. In 2017-2018, participants were invited to complete the second questionnaire. Both questionnaires retrospectively enquired about chlamydia infections, pregnancy intention, pregnancies, and time to pregnancy in months. Additionally, demographic factors were collected such as migration background and education level. Migration background was classified as 'Western' if both parents had a Western country of birth i.e. a country from Europe [excluding Turkey], North America, Oceania, Indonesia and 
Japan, 'non-Western' if at least one parent had a non-Western country of birth, and 'unknown' if one parent was Western while the country of birth of the other parent was unknown or when the country of birth of both parents was unknown. Education level was defined as low/medium: no education, primary education only, lower general secondary education and vocational education; and high: higher professional education and university education) at last completed questionnaire. Furthermore, sexual behavior, other STIs, contraceptive use, and health characteristics were addressed. For all events (i.e. chlamydia infections, gonorrhea infections, and pregnancies), timing in calendar year was requested in order to reconstruct a timeline. Subsequently, CSI data about chlamydia NAAT results, self-reported chlamydia infections, and age of sexual debut, were merged with NECCST data.

\section{Definitions}

Chlamydia status: Women were defined as chlamydia-positive if they were either chlamydiaNAAT positive in CSI, chlamydia-positive by IgG antibody presence, or self-reported an infection during CSI or NECCST; women were considered positive from the year of first infection onwards. If women were tested negative by NAAT, negative for chlamydia antibodies, and did not report a previous infection, they were defined as chlamydia-negative.

Overall pregnancies: Pregnancy was defined as a self-reported pregnancy, which also included miscarriages and induced abortions. Overall pregnancies included planned as well as unplanned pregnancies, and irrespective of fertility treatments.

Women with a pregnancy intention: This includes all women who had at least one planned pregnancy (irrespective of fertility treatments) or who had ever tried to conceive or were still trying. Time period of the cohort: Women were first invited for the CSI study between 2008 and 2011. Women were invited for NECCST in 2015 or 2016 and re-invited in 2017 or 2018. The cohort period was shortest from 2011-2015 and longest from 2008-2018. The last received questionnaire was dated 8/21/2018.

Exposure time in Cox regression was defined in two ways.

- $\quad$ First, to assess the chance of getting pregnant for chlamydia-positive women compared to chlamydia-negative women, exposure time was defined as the years since sexual debut until last completed questionnaire. This starting point was chosen because from sexual debut on, women were 'at risk' for chlamydia infection and (unplanned) pregnancies.

- Second, to determine the chance of getting pregnant over time for chlamydia-positive women compared to chlamydia-negative women, time to pregnancy was defined as the number of months women attempted to become pregnant until first planned pregnancy, until she stopped attempting to get pregnant, or until the last completed questionnaire.

\section{Statistical analyses}

Population characteristics, pregnancy proportions and time to pregnancy were compared between chlamydia-positive women and chlamydia-negative women using student t test, Mann-Whitney $\mathrm{U}$ tests, and chi-square tests. In addition, pregnancy incidence rates (calculated as the number of pregnancies divided by total person-years at risk) were given. 


\section{- Overall pregnancies}

The association between chlamydia and getting pregnant was assessed through a KaplanMeier curve and Cox regression among all participants. To include multiple pregnancies per participant, we used recurrent event analyses based on the method of Prentice, Williams and Peterson, which is known as the conditional risk set model. In this model, the assumption is that a subject is not at risk of a second event until the first event has occurred, and so on(16). Risks were expressed in (adjusted) hazard ratios ((a)HR). Chlamydia status was included as a time-varying variable. Multiple imputations were done to estimate the time of first chlamydia infection if this was unknown, i.e. in women with a positive chlamydia IgG result only (a positive IgG result can be a result of an infection from years earlier). With STATA's multiple imputation command using truncated regression analyses with 10 simulation datasets $(17,18)$ time of first chlamydia infection following sexual debut was estimated based on available data from women with a known year of first infection. This was done for 222 women (4\% of all participants and $13 \%$ among chlamydia-positive women). The proportional-hazard $(\mathrm{PH})$ assumption was checked using log-log plots and testing Schoenfeld residuals. Effect modification, to identify whether the effect of chlamydia infection was different in groups with different characteristics, was assessed for the following variables: age, sex of the partner, and migration background. In the case of significant effect modification, analyses were stratified. The following variables were considered potential confounders and included in the model if there was a $\geq 10 \%$ change in the regression coefficient: age (time-varying), migration background, educational level. Additionally, smoking behavior, and BMI category at start of NECCST, gonorrhea infection, number of lifetime sex partners, age of sexual debut, and condom use with casual partner were considered potential confounders.

\section{- Women with a pregnancy intention}

Timing in months until the first planned pregnancy and chlamydia status was also assessed by Kaplan-Meier curves and Cox regression. All women with a pregnancy intention were included in this analysis. The PH assumption and effect modification were assessed as described above. Chlamydia status was negative in the case of no positive test outcome or in the case that a first planned pregnancy occurred before the first chlamydia diagnosis. Chlamydia status was defined as positive when the first infection occurred before the first planned pregnancy. The following potential confounders at time of trying to get pregnant, in addition to confounders described above, were evaluated: age, BMI, last contraceptive, and smoking behavior. Contraceptive use was categorized as: No contraceptive use, Hormonal contraceptives, Mirena, Copper IUD, or Condoms.

\section{Sensitivity analyses}

Various sensitivity analyses were performed as described in Table 1. 
Table 1. Sensitivity analyses

\begin{tabular}{|c|c|}
\hline & Sensitivity analyses \\
\hline 1 & $\begin{array}{l}\text { Analyses were repeated without multiple imputations, i.e. women without a known year of first } \\
\text { chlamydia infection were excluded }\end{array}$ \\
\hline 2 & $\begin{array}{l}\text { Analyses were repeated selecting CSI-NAAT-positive women versus chlamydia-negative women, } \\
\text { due to high sensitivity and specificity and no recall bias using CSI-NAAT results. In addition, to } \\
\text { understand the impact of measurement bias, an analysis was performed where chlamydia was } \\
\text { classified as NAAT negative, NAAT positive, serology positive, or self-reported positive. }\end{array}$ \\
\hline 3 & $\begin{array}{l}\text { The analysis was restricted to women who participated in both the first and second questionnaire } \\
\text { round of NECCST to test for selection bias response. }\end{array}$ \\
\hline 4 & $\begin{array}{l}\text { Analyses were repeated where we excluded women with a first chlamydia infection that occurred } \\
\text { in the same year as a first pregnancy because of the possibility that the infection occurred after } \\
\text { the pregnancy rather than before. }\end{array}$ \\
\hline 5 & $\begin{array}{l}\text { Analyses were repeated where we excluded women who had their first infection diagnosed after } \\
\text { a planned pregnancy }\end{array}$ \\
\hline 6 & $\begin{array}{l}\text { Analyses were repeated in which chlamydia status was categorized as chlamydia-negative, one } \\
\text { infection or multiple chlamydia infections }\end{array}$ \\
\hline 7 & $\begin{array}{l}\text { Last, analyses were repeated in which chlamydia status was categorized as chlamydia-negative, } \\
\text { chlamydia-positive or chlamydia-seropositive-only. }\end{array}$ \\
\hline
\end{tabular}

CSI $=$ chlamydia screening implementation. NAAT $=$ Nucleic Acid Amplification Test. NECCST $=$ Netherlands Chlamydia Cohort Study.

\section{Medical ethical approval}

This study was approved by the Medical Ethical Committee VU medical Center, Amsterdam, the Netherlands (NL 51553.094.14/2015.903(A2019.336)). All participants provided informed consent.

\section{Results}

In total, 5,704 women were enrolled in NECCST and completed the initial questionnaire. In $2017 / 2018,5,676$ (99.5\%) ( $n=28$ study withdrawals) women were invited to complete the second NECCST questionnaire, of whom 3,622 (63.5\%) did, see Figure 1. Total exposure time from sexual debut until last questionnaire was 93,854 person-years.

\section{Study population}

Characteristics of the study population by chlamydia status at last data collection round (either the first or second NECCST questionnaire round) are presented in Table 2. Mean age was 32.0 years (SD 3.9). Eighty percent of women had a high educational level and a Western migration background. Thirty percent of women were chlamydia-positive. Compared to chlamydia-negative women, chlamydia-positive women were younger at sexual debut, had more lifetime sex partners, had a history of gonorrhea more often, and were more often smokers. 
Table 2. Study population characteristics by chlamydia status at timing of last data collection moment

\begin{tabular}{|c|c|c|c|c|}
\hline & $\begin{array}{r}\text { Overall } \\
\mathrm{n}(\%)\end{array}$ & $\begin{array}{r}\text { Chlamydia-negative } \\
\mathrm{n}(\%)\end{array}$ & $\begin{array}{r}\text { Chlamydia-positive } \\
\mathrm{n}(\%)\end{array}$ & P-value \\
\hline Overall & $5704(100.0)$ & 3987 (69.9) & $1717(30.1)$ & \\
\hline \multicolumn{5}{|l|}{ Chlamydia status } \\
\hline Negative & 3987 (69.9) & 3987 (100.0) & $0(0.0)$ & $<0.01$ \\
\hline \multicolumn{5}{|l|}{ Positive by category: } \\
\hline Self-reported & $900(15.8)$ & $0(0.0)$ & $900(52.4)$ & \\
\hline CSI-NAAT & $4(0.1)$ & $0(0.0)$ & $4(0.2)$ & \\
\hline Chlamydia IgG & $208(3.7)$ & $0(0.0)$ & $208(12.1)$ & \\
\hline Self-reported + CSI-NAAT & $244(4.3)$ & $0(0.0)$ & $244(14.2)$ & \\
\hline Self-reported + chlamydia lgG & $263(4.6)$ & $0(0.0)$ & $263(15.3)$ & \\
\hline CSI-NAAT + chlamydia IgG & $1(0.0)$ & $0(0.0)$ & $1(0.1)$ & \\
\hline All positive & $97(1.7)$ & $0(0.0)$ & $97(5.7)$ & \\
\hline Age (years) mean (SD) & $32.4(3.9)$ & $32.5(3.9)$ & $32.1(3.9)$ & $<0.01$ \\
\hline \multicolumn{5}{|l|}{ Migration background } \\
\hline Western & $4565(80.0)$ & $3354(84.1)$ & $1211(70.5)$ & $<0.01$ \\
\hline Non-Western & 869 (15.2) & $458(11.5)$ & $411(23.9)$ & \\
\hline Unknown & $270(4.7)$ & $175(4.4)$ & $95(5.5)$ & \\
\hline \multicolumn{5}{|l|}{ Educational level ${ }^{\$ \#}$} \\
\hline Low/middle & $1163(20.4)$ & $648(16.3)$ & $515(30.0)$ & $<0.01$ \\
\hline High & $4539(79.6)$ & $3338(83.7)$ & $1201(70.0)$ & \\
\hline \multicolumn{5}{|l|}{ Age at sexual debut mean (SD) } \\
\hline$<16$ & $1558(27.3)$ & $956(24.0)$ & $602(35.1)$ & $<0.01$ \\
\hline $16-17$ & $2178(38.2)$ & $1507(37.8)$ & $674(30.8)$ & \\
\hline$>17$ & $1968(34.5)$ & $1524(38.2)$ & $444(25.9)$ & \\
\hline \multicolumn{5}{|l|}{ Lifetime sex partners } \\
\hline$<6$ & $1306(22.9)$ & 1077 (27.0) & 229 (13.3) & $<0.01$ \\
\hline $6-12$ & $2174(38.1)$ & $1598(40.1)$ & 576 (33.6) & \\
\hline$>12$ & 2224 (39.0) & 1312 (32.9) & $912(53.1)$ & \\
\hline \multicolumn{5}{|l|}{ Condom use with casual partners +} \\
\hline Never/not often & $340(6.0)$ & $242(6.1)$ & $98(5.7)$ & $<0.01$ \\
\hline Sometimes & 1807 (31.7) & $1081(27.2)$ & 726 (42.3) & \\
\hline Always/mostly & 2610 (45.9) & $1876(47.2)$ & $734(42.8)$ & \\
\hline No casual partners & $936(16.4)$ & $779(19.6)$ & $157(9.2)$ & \\
\hline Gonorrhea positivity n (\%) & $120(2.1)$ & $36(0.9)$ & $84(4.9)$ & $<0.01$ \\
\hline \multicolumn{5}{|l|}{ Use of IUD } \\
\hline Never & 3507 (61.5) & $2471(62.0)$ & $1036(60.3)$ & 0.24 \\
\hline At least once & 2197 (38.5) & $1516(38.0)$ & $681(39.7)$ & \\
\hline \multicolumn{5}{|l|}{ Smoking } \\
\hline Never & 2260 (39.6) & $1712(42.9)$ & 548 (31.9) & $<0.01$ \\
\hline Sometimes/in the past & $2919(51.2)$ & $1981(49.7)$ & 938 (54.6) & \\
\hline Daily & $525(9.2)$ & $294(7.4)$ & $231(13.5)$ & \\
\hline \multicolumn{5}{|l|}{$\mathrm{BMI} \&$} \\
\hline$<20$ & $730(12.8)$ & $536(13.5)$ & $194(11.3)$ & $<0.01$ \\
\hline $20-<25$ & $3448(60.6)$ & 2437 (61.2) & $1011(59.0)$ & \\
\hline $25-<30$ & $1052(18.5)$ & $713(17.9)$ & $339(19.8)$ & \\
\hline$\geq 30$ & $464(8.2)$ & $294(7.4)$ & $170(9.9)$ & \\
\hline
\end{tabular}

Chlamydia-positive was defined as a positive NAAT test outcome in the CSI study (CSI-NAAT), and/or the presence of chlamydia IgG or a self-reported chlamydia infection. \#At start of NECCST and based on 5702 observations, 2 missing. \$Educational level: Low/medium level of education: no education, primary education only, lower general secondary education and vocational education; high level of education: all other education levels. +Based on 5693 observations, 11 missing and based on first questionnaire data. "based on 5694 observations, 10 missing. SD = standard deviation. $\mathrm{CSI}=$ chlamydia screening implementation. NAAT = Nucleic Acid Amplification Test. IUD = Intrauterine Device. IgG = Immunoglobulin G. BMI = Body mass index. * = statistically significant, $\mathrm{p}<0.05$. 


\section{Overall pregnancies}

In total, 3,146 (55.2\%) women (ever) tried to become pregnant or were pregnant at least once, see Table 3. Mean age at first pregnancy was 29.4 (95\%Cl 29.2-29.6). There was no significant difference in percentage of women who had ever tried/were trying to become pregnant, had been pregnant, or had never tried to become pregnant between chlamydia-negative women and chlamydia-positive women $(p=0.316)$.

Pregnancy incidences were described in Table 3. The Kaplan-Meier curve is presented in Figure 2A. Time was censored at 23 years after sexual debut due to sample size reduction. The Schoenfeld residuals test $(p=0.001)$ indicated that the chance ratio of getting pregnant was not proportional over time. Therefore, to calculate hazard ratios, the exposure time was stratified in four intervals based on graphs and testing the $\mathrm{PH}$ assumption. In the first two exposure time intervals of 0-6 years and 7-10 years, chances for pregnancy were higher in chlamydia-positive women compared to chlamydia-negative women: aHR $1.54(95 \% \mathrm{Cl} 1.26-1.89)$ and aHR $1.42(95 \% \mathrm{Cl} 1.22-1.66)$ respectively. In the two last exposure time categories of $11-13$ years and $13-23$ years, no differences were seen: aHR 0.93 (95\%Cl 0.80-1.07) and aHR 1.00 (95\%Cl 0.90-1.10), see Table 4.

Table 3. Pregnancies in chlamydia-negative and chlamydia-positive women

\begin{tabular}{|c|c|c|c|c|c|c|c|c|c|}
\hline \multirow[b]{2}{*}{ Never pregnant nor tried } & \multicolumn{3}{|c|}{$\begin{array}{c}\text { All women } \\
\text { N, \% }(95 \% \mathrm{Cl})\end{array}$} & \multicolumn{3}{|c|}{$\begin{array}{c}\text { Chlamydia-negative } \\
\text { women } \\
\text { N, \% }(95 \% \mathrm{Cl})\end{array}$} & \multicolumn{3}{|c|}{$\begin{array}{c}\text { Chlamydia-positive } \\
\text { women } \\
\text { N, \% }(95 \% \mathrm{Cl})\end{array}$} \\
\hline & 2558 & 44.9 & $(44-46)$ & 1821 & 44.7 & $(43.1-46.2)$ & 737 & 45.3 & $(42.9-47.8)$ \\
\hline $\begin{array}{l}\text { Tried or trying and failed to } \\
\text { become pregnant }\end{array}$ & 291 & 5.1 & $(4.5-5.7)$ & 198 & 4.9 & $(4.2-5.6)$ & 93 & 5.7 & $(4.6-7.0)$ \\
\hline Ever pregnant* & 2855 & 50.1 & $(48.7-51.4)$ & 2058 & 50.5 & $(48.9-52.0)$ & 797 & 49.0 & $(46.5-51.4)$ \\
\hline Planned (at least once) & 2051 & 71.8 & $(70.1-73.5)$ & 1558 & 75.7 & $(73.8-77.5)$ & 493 & 61.9 & 58.4-65.2) \\
\hline $\begin{array}{l}\text { Pregnancies per } 1000 \\
\text { person-years }\end{array}$ & & 58.4 & $(56.7-60.0)$ & & 53.2 & $(51.5-55.0)$ & & 83.0 & $(78.5-87.9)$ \\
\hline
\end{tabular}

* 35 women who had a previous unplanned pregnancy were also trying to get pregnant without success yet. In this table they are included in the ever pregnant category. 
Table 4. Association between chlamydia status and getting pregnant by exposure time intervals

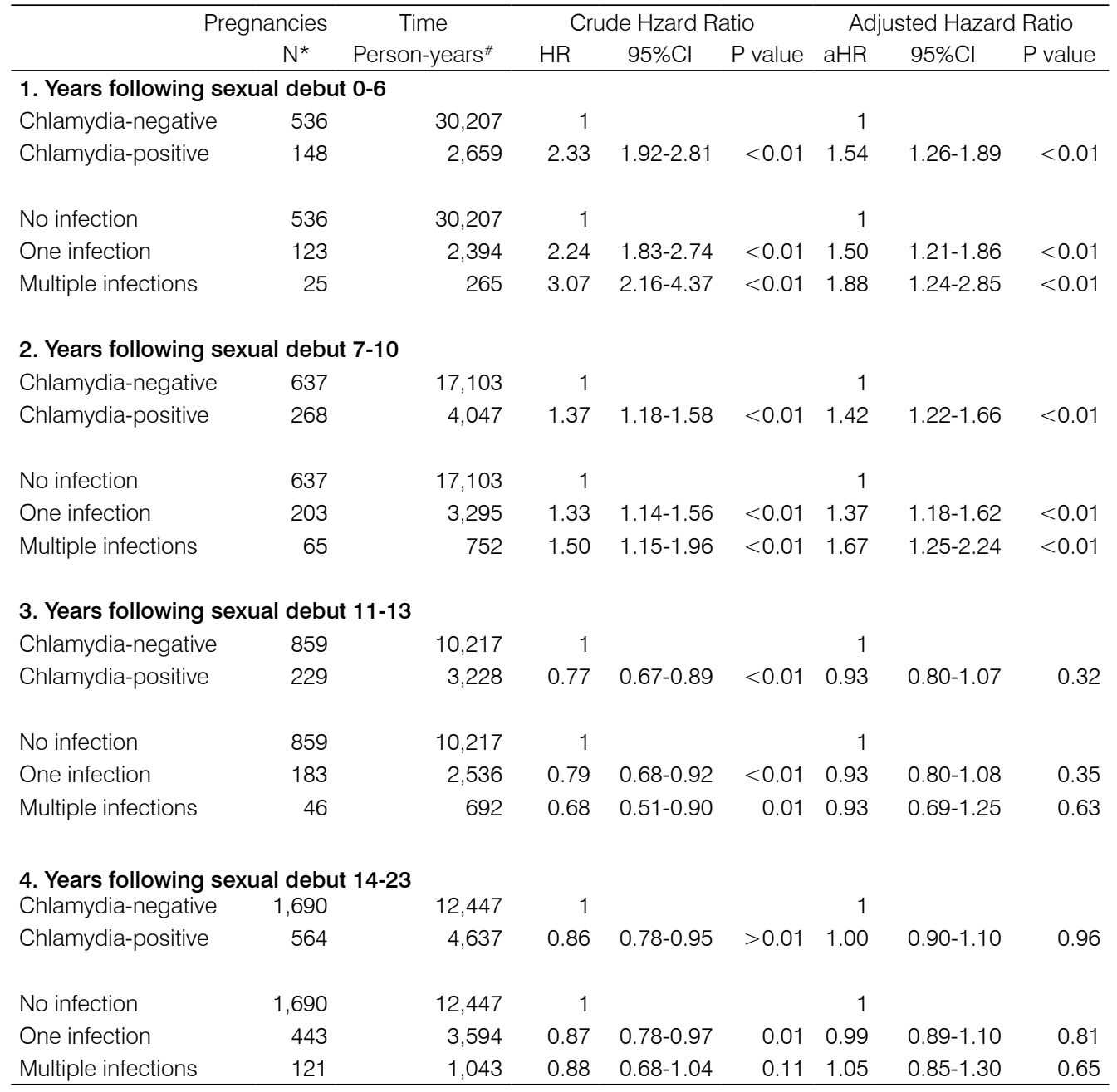

Chlamydia-positive was defined as a positive NAAT-test outcome in the CSI study (CSI-NAAT), and/or the presence of chlamydia IgG and/or a self-reported chlamydia infection. For these analyses, multiple imputations were used to estimate time of first chlamydia infection in women without a known first year of chlamydia infection. ${ }^{\star}$ Complete cases. ${ }^{\# P e r s o n-y e a r s ~ o f ~ c o m p l e t e ~ c a s e s . ~ M o d e l s ~ w e r e ~ a d j u s t e d ~ f o r ~}$ age, education level, migration background and number of lifetime partners. $\mathrm{HR}=$ Hazard Ratio. $\mathrm{Cl}=$ Confidence Interval. aHR = adjusted Hazard Ratio.

\section{Women with a pregnancy intention}

In total, 2,377 (41.7\%) women had ever attempted to conceive. After excluding records with missing values in time $(n=29)$ and with an unclear number of pregnancies $(n=33), 2,315$ women were included in the analyses. In total, the pregnancy proportion was $87.4 \%$. Overall $83.3 \%$ became pregnant within 12 months. This was $85.0 \%$ (95\% Cl 83.3-86.7) among chlamydia-negative women 
and $77.9 \%(95 \% \mathrm{Cl} 73.9-81.5)$ among chlamydia-positive women. Of the remaining chlamydianegative women, $7.7 \%$ had a time to pregnancy $>12$ months and the remaining $7.3 \%$ had time $<13$ months but did not yet conceive. For chlamydia positives this was $11.6 \%$ and $10.7 \%$ respectively. Median (IQR) and mean (SD) time to pregnancy was 3 (IQR 1-6) months and 5.6 (SD 9.0) months for chlamydia negatives and 3 (IQR 1-7) months and 6.7 (SD 11.0) months for chlamydia positives, $\mathrm{p}=0.007$ (based on Mann-Whitney $U$ test). The overall pregnancy rate per py was $1.8(95 \% \mathrm{Cl} 1.7-1.9) ; 1.9(95 \% \mathrm{Cl} 1.8-2.0)$ for chlamydia-negative women and $1.5(95 \% \mathrm{Cl}$ 1.3-1.6) for chlamydia-positive women. Figure 2B shows the Kaplan-Meier curve. Time was censored at 36 months due to sample size reduction. In Cox regression analyses, stratified for age due to effect modification by age (i.e. age was divided into tertiles), chances of pregnancy for chlamydia-positive women were lower compared to chlamydia-negative women in the age categories $16-29$ years and $30-32$ years (aHR 0.79, 95\% Cl 0.67-0.94 and aHR 0.74, 95\%Cl 0.600.92 , respectively), Table 5. In age category 33-39 years, no significant difference was found (aHR 1.07, 95\% Cl 0.89-1.28).

Table 5. Association between chlamydia status and getting pregnant among women with a pregnancy intention stratified by age at time of attempting to conceive

\begin{tabular}{|c|c|c|c|c|c|c|c|c|}
\hline & \multirow{2}{*}{$\begin{array}{c}\text { Planned } \\
\text { pregnancies } \\
\mathrm{N}^{*}\end{array}$} & \multirow{2}{*}{$\begin{array}{l}\text { Time } \\
\text { Person- } \\
\text { months }{ }^{\#}\end{array}$} & \multicolumn{3}{|c|}{ Crude Hazard Ratio } & \multicolumn{3}{|c|}{ Adjusted Hazard Ratio } \\
\hline & & & $\mathrm{HR}$ & $95 \% \mathrm{Cl}$ & $P$ value & $\mathrm{aHR}$ & $95 \% \mathrm{Cl}$ & $P$ value \\
\hline \multicolumn{9}{|l|}{ 1. Age $16-29$} \\
\hline Chlamydia-negative & 709 & 3,871 & 1 & & & 1 & & \\
\hline Chlamydia-positive & 162 & 1,374 & 0.78 & $0.66-0.92$ & $<0.01$ & 0.79 & $0.67-0.94$ & $<0.01$ \\
\hline No infection & 709 & 3,871 & 1 & & & 1 & & \\
\hline One infection & 132 & 1,069 & 0.80 & $0.67-0.97$ & 0.01 & 0.81 & $0.68-0.97$ & 0.02 \\
\hline Multiple infections & 30 & 305 & 0.71 & $0.49-1.03$ & 0.07 & 0.71 & $0.49-1.03$ & 0.07 \\
\hline \multicolumn{9}{|l|}{ 2. Age $30-32$} \\
\hline Chlamydia-negative & 425 & 2,162 & 1 & & & 1 & & \\
\hline Chlamydia-positive & 98 & 692 & 0.72 & $0.59-0.89$ & $<0.01$ & 0.74 & $0.60-0.92$ & $<0.01$ \\
\hline No infection & 425 & 2,162 & 1 & & & 1 & & \\
\hline One infection & 79 & 571 & 0.72 & $0.58-0.90$ & $<0.01$ & 0.75 & $0.59-0.94$ & 0.01 \\
\hline Multiple infections & 19 & 121 & 0.71 & $0.45-1.13$ & 0.15 & 0.72 & $0.45-1.14$ & 0.16 \\
\hline \multicolumn{9}{|l|}{ 3. Age $33-39$} \\
\hline Chlamydia-negative & 485 & 3649 & 1 & & & 1 & & \\
\hline Chlamydia-positive & 131 & 842 & 1.07 & $0.89-1.28$ & 0.48 & 1.07 & $0.89-1.28$ & 0.49 \\
\hline No infection & 485 & 3649 & 1 & & & 1 & & \\
\hline One infection & 115 & 690 & 1.11 & $0.92-1.34$ & 0.29 & 1.10 & $0.90-1.33$ & 0.35 \\
\hline Multiple infections & 16 & 152 & 0.81 & $0.49-1.34$ & 0.42 & 0.85 & $0.50-1.42$ & 0.52 \\
\hline
\end{tabular}

Chlamydia-positive was defined as a positive NAAT-test outcome in the CSI study (CSI-NAAT), and/or the presence of chlamydia lgG and/or a self-reported chlamydia infection. For these analyses, multiple imputations were used to estimate time of first chlamydia infection in women without a known first year of chlamydia infection. Analyses were stratified for age categories based on tertiles. ${ }^{\star}$ Complete cases. \#Person-months of complete cases. All models were adjusted for age at pregnancy/trying to get pregnant and migration background. $\mathrm{HR}=$ Hazard Ratio. $\mathrm{Cl}=$ Confidence Interval. aHR = adjusted Hazard Ratio. 
A

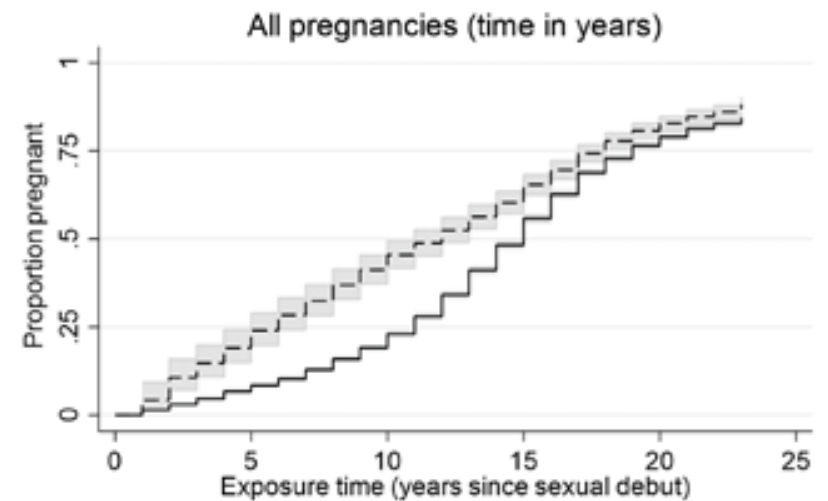

Number at risk

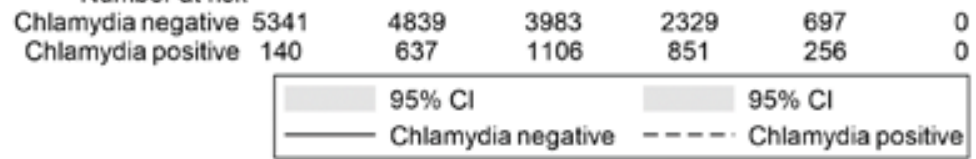

B
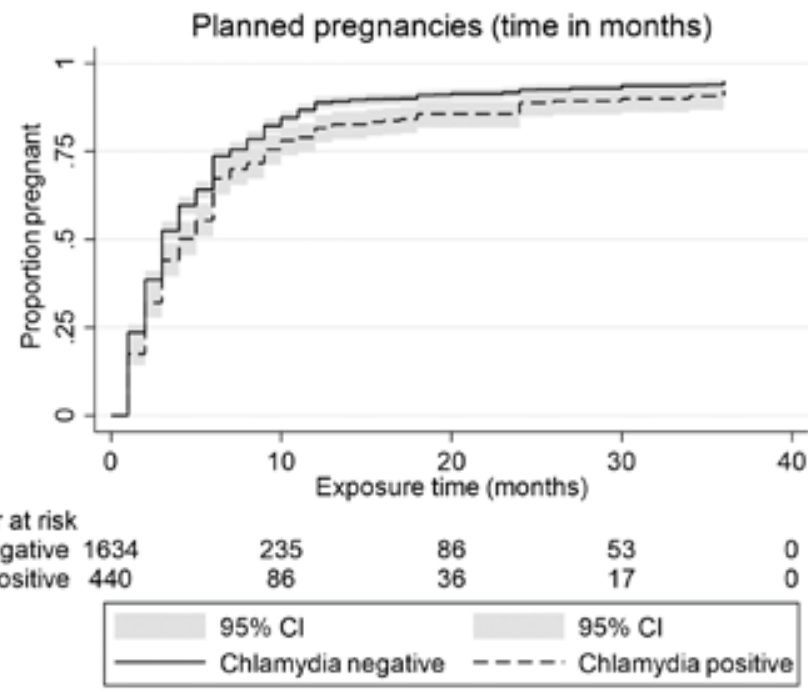

Number at risk

Chlamydia negative 1634

Chlamydia positive 440

86

Chlamydia positive

Figure 2. Kaplan-Meier plots of years since sexual debut until first pregnancy (A) and time to a planned pregnancy in months among women with a pregnancy intention (B) by chlamydia status. Chlamydia-positive was defined as a positive NAAT-test outcome in the CSI study (CSI-NAAT), and/or the presence of chlamydia $\lg \mathrm{G}$ and/or a self-reported chlamydia infection.

Sensitivity analyses

Sensitivity analyses on pregnancies and time to pregnancy using data restrictions as indicated in the methodology section yielded comparable results, see Supplementary Figures 1 and 2, and Supplementary tables 1 and 2 . 


\section{Discussion}

In this longitudinal cohort study with over ten years follow-up, we assessed pregnancies and time to pregnancy among chlamydia-positive and chlamydia-negative women. Overall pregnancy proportions (either planned or unplanned) in chlamydia-positive and chlamydia-negative women were relatively similar. More chlamydia-positive women were pregnant in the first ten years following sexual debut, but this difference decreased thereafter. By contrast, among women below 33 years of age with a pregnancy intention, pregnancy rates were lower in chlamydia-positive women compared to chlamydia-negative women and time to pregnancy was longer. No differences in pregnancy rates were seen after 33 years of age.

This unique cohort is a follow-up study from the population-based CSI and included information about previous chlamydia infections based on NAAT results, chlamydia IgG presence, and self-reported positive test results, thereby reducing misclassification. However, in common with other chlamydia studies it is inevitable that there is misclassification in chlamydia status. NAAT tests can only detect current infections, not past infections and these were performed only once per year in the CSI(2). Serology can identify past infections, but sensitivity is not optimal and serology was only done in the first NECCST questionnaire round, not in the second $(19,20)$. Furthermore, participants were classified as 'chlamydia negative' (until first positive test), which could have been before first test outcome. Last, self-reported infections may be subjected to recall bias. To understand the impact of measurement bias, several sensitivity analyses were performed that yielded similar results. NECCST is a subset of the population-based CSI study, and selection bias cannot be ruled out. Participants were more often highly educated and from a Western migration background than the general Dutch population. However, our pregnancy results are quite comparable with the general Dutch population. The average age of women when having their first child in the Netherlands in 2013 (i.e. the median year of first pregnancy in this cohort) was 29.4, which is equal to the age of first pregnancy in this cohort. Furthermore, in the Netherlands, about $83 \%$ of women who wish to become pregnant conceive within one year, as similarly reported by $82 \%$ of women in this study.

In contrast to our hypothesis, overall pregnancy proportions were rather comparable between chlamydia-positive and chlamydia-negative women - both about $50 \%$ in the total cohort. We expected lower pregnancy proportions because several studies, including a previous study of this cohort, demonstrated a 1.3-4 times increased risk for TFI following chlamydia infection(21-23). Our results are, however, in agreement with population-based cohort studies from Denmark and Norway, in which no differences were seen in birth rates between chlamydia-seropositive women and chlamydia-seronegative-tested women(12). In the population-based study from Denmark, a slightly higher chance of births was seen for chlamydia-positive women, either chlamydiaseropositive or by a positive chlamydia DNA test(11). In a clinical study in Iran in which 250 infertile couples were tested for chlamydia DNA and chlamydia serology, no difference in pregnancy chances was found either(24). How can this be explained? Although 1.3-4 times higher compared to chlamydia-negative women, the risk for TFI following chlamydia infection is low $(0.5-1.0 \%)$ (7). This effect might get lost in large cohorts in which many other factors for getting pregnant 
played a role. We did not have data about frequency of sexual contacts, which may have had an impact. We found that unplanned pregnancies were significantly more common (14\% more) among chlamydia-positive women compared to chlamydia-negative women. As both are linked to unprotected sexual contact, this is not unexpected(25). Despite not having information about sexual frequency, our results imply that sexual activity among chlamydia-positive women could have been higher i.e. significantly more lifetime partners, younger age at sexual debut, and more previous gonorrhea infections. In the Netherlands, there is no antenatal chlamydia screening, but it might be that part of unplanned pregnancies were terminated in abortion clinics were women were offered a chlamydia test. More chlamydia tests could have been done which might have led to an association between chlamydia positivity and pregnancy. However abortion rates in the Netherlands are low (8.8 per 1000 women in 2018) and only eight percent of abortions were done among teenagers(26). Nevertheless, these factors in combination with less condom use might have resulted in more pregnancies and explain why more chlamydia-positive women were pregnant within the first ten years following sexual debut.

By contrast, among women with a pregnancy intention, pregnancy proportions and rates per year were lower among chlamydia-positive women. These lower rates can only partly be explained by the known TFI cases, which is only $1 \%$ in this cohort. Lower pregnancy proportions were also seen in the study following infertile women with and without chlamydia antibodies where fewer chlamydia-antibody-positive women became pregnant within nine months(27). In that study, other causes of infertility including visible TFI were excluded. The possibility is that even without visible tubal pathology, there might be intratubal microdamage due to a previous chlamydia infection(27). On the other hand, we cannot completely rule out selection bias for the effect. These lower pregnancy rates were found among women with a pregnancy intention. In this analyses, all women who only had an unplanned pregnancy were excluded. In the questionnaire, women were asked if reported pregnancies were 'planned' without the definition of 'planned' being explained further. A planned pregnancy could have been interpreted differently between women and wrongly classified as 'unplanned', which might explain our high rates of unplanned pregnancies. Although excluding unplanned pregnancies led to more clear analyses, a bias might be introduced because more chlamydia-positive women had unplanned pregnancies, which could have either reduced or underestimated the effect.

It is difficult to put our results in the context of chlamydia control. In the Netherlands, extensive chlamydia control efforts are being made. Youngsters up to the age of 25 and high-risk groups can be tested and treated for chlamydia free of charge(28). It is possible that because of the extensive testing and treating of chlamydia infections, pregnancy rates are not lower in chlamydia-positive women compared with chlamydia-negative women. However, we did see lower pregnancy rates among women with a pregnancy intention. In sensitivity analyses in which we estimated risks for women who were positive only by a chlamydia antibody test and had not reported a chlamydia infection, and thus were presumably not treated for chlamydia, pregnancy rates did not differ from chlamydia positives, of which the vast majority had been treated. Although the group of chlamydia-positive women who were presumably not treated for their infection was rather small, it is possible that the effect of chlamydia control in preventing late complications that might result 
in infertility is rather small(29).

Using pregnancy rates and time to pregnancy in addition to PID, ectopic pregnancy, and TFI to study reproductive health outcomes following chlamydia infections contributes to the insight in chlamydia disease burden. Pregnancy characteristics are easy to collect with high validity using questionnaires(30). Nevertheless, in using questionnaires to address events dating back to years ago, recall bias cannot be ruled out. Given that in our study the median number of years between first infection and first pregnancy was five, we assume that not many first pregnancies were incorrectly reported before first infection, however this possibility should be taken into account in interpreting the results.

To conclude, our results do not indicate that women with a previous chlamydia infection have lower pregnancy chances compared to women without a chlamydia infection. However, we did find lower pregnancy rates and longer time to pregnancy among women with a pregnancy intention, which could be due to complications of the chlamydia infection caused by either visible or invisible tubal pathology. It is worthwhile to not only focus on chlamydia complications but to also include pregnancy as an outcome in surveillance and research. 


\section{References}

1. Heijne JCM, van den Broek IVF, Bruisten SM, van Bergen JEA, de Graaf $H$, van Benthem BHB. National prevalence estimates of chlamydia and gonorrhoea in the Netherlands. Sex Transm Infect. 2019;95(1):53-9.

2. van den Broek IV, van Bergen JE, Brouwers $\mathrm{EE}$, et al. Effectiveness of yearly, register based screening for chlamydia in the Netherlands: controlled trial with randomised stepped wedge implementation. BMJ. 2012;345:e4316.

3. van Bergen J, Gotz $\mathrm{H}$, Richardus $\mathrm{JH}$, et al. Prevalence of urogenital Chlamydia trachomatis infections in the Netherlands suggests selective screening approaches. Results from the PILOT CT Population Study. Drugs Today (Barc). 2006;42 Suppl A:25-33.

4. Haggerty CL, Gottlieb SL, Taylor BD, Low N, Xu $F$, Ness RB. Risk of sequelae after Chlamydia trachomatis genital infection in women. J Infect Dis. 2010;201 Suppl 2:S134-55.

5. Hafner LM. Pathogenesis of fallopian tube damage caused by Chlamydia trachomatis infections. Contraception. 2015;92(2):108-15.

6. van der Steeg JW, Steures P, Eijkemans MJ, et al. Predictive value of pregnancy history in subfertile couples: results from a nationwide cohort study in the Netherlands. Fertil Steril. 2008;90(3):521-7.

7. Price MJ, Ades AE, Soldan K, et al. The natural history of Chlamydia trachomatis infection in women: a multi-parameter evidence synthesis. Health Technol Assess. 2016;20(22):1-250.

8. Hoenderboom BM, van Benthem BHB, van Bergen $\mathrm{J}$, et al. Relation between Chlamydia trachomatis infection and pelvic inflammatory disease, ectopic pregnancy and tubal factor infertility in a Dutch cohort of women previously tested for chlamydia in a chlamydia screening trial. Sex Transm Infect. 2019.

9. Kamel RM. Management of the infertile couple: an evidence-based protocol. Reprod Biol Endocrinol. 2010;8:21.

10. McLernon DJ, Maheshwari A, Lee AJ,
Bhattacharya S. Cumulative live birth rates after one or more complete cycles of IVF: a population-based study of linked cycle data from 178,898 women. Hum Reprod. 2016;31(3):572-81.

11. Bakken IJ, Skjeldestad FE, Lydersen S, Nordbo SA. Births and ectopic pregnancies in a large cohort of women tested for Chlamydia trachomatis. Sex Transm Dis. 2007;34(10):73943.

12. Andersen B, Ostergaard L, Puho E, Skriver MV, Schonheyder HC. Ectopic pregnancies and reproductive capacity after Chlamydia trachomatis positive and negative test results: a historical follow-up study. Sex Transm Dis. 2005;32(6):377-81.

13. Hoenderboom BM, van Oeffelen AA, van Benthem $\mathrm{BH}$, et al. The Netherlands Chlamydia cohort study (NECCST) protocol to assess the risk of late complications following Chlamydia trachomatis infection in women. BMC Infect Dis. 2017;17(1):264

14. Morre SA, Munk C, Persson K, et al. Comparison of Three Commercially Available Peptide-Based Immunoglobulin $G(\mathrm{lg} G)$ and $\lg A$ Assays to Microimmunofluorescence Assay for Detection of Chlamydia trachomatis Antibodies. Journal of Clinical Microbiology. 2002;40(2):584-7.

15. Hoenderboom BM, van Ess EF, van den Broek IVF, et al. Chlamydia trachomatis antibody detection in home-collected blood samples for use in epidemiological studies. J Microbiol Methods. 2017;144:164-7.

16. Prentice RL, Williams, B.J., Peterson, A.V. On the regression analysis of multivariate failure time data. Biometrika. 1981;68(2):373-9.

17. Raghunathan TE, Lepkowski, J.M., Van Hoewyk, J., Solenberger, P.,. A Multivariate Technique for Multiply Imputing Missing Values Using a Sequence of Regression Models. Survey Methodology. 2001;27(No. 1):85-95.

18. StataCorp. Stata: Release 13. Statistical Software. College Station, TX: StataCorp LP.; 
2013.

19. Morre SA, Munk C, Persson K, et al. Comparison of three commercially available peptide-based immunoglobulin $G$ ( $(\mathrm{gG})$ and $\lg A$ assays to microimmunofluorescence assay for detection of Chlamydia trachomatis antibodies. J Clin Microbiol. 2002;40(2):584-7.

20. Horner PJ, Wills GS, Reynolds R, et al. Effect of time since exposure to Chlamydia trachomatis on chlamydia antibody detection in women: a cross-sectional study. Sex Transm Infect. 2013;89(5):398-403.

21. Davies B, Turner KME, Frølund M, et al. Risk of reproductive complications following chlamydia testing: a population-based retrospective cohort study in Denmark. The Lancet Infectious Diseases. 2016;16(9):1057-64.

22. Hoenderboom BM, van Benthem BHB, van Bergen $\mathrm{J}$, et al. Relation between Chlamydia trachomatis infection and pelvic inflammatory disease, ectopic pregnancy and tubal factor infertility in a Dutch cohort of women previously tested for chlamydia in a chlamydia screening trial. Sex Transm Infect. 2019;95(4):300-6.

23. Low N, Egger M, Sterne JA, et al. Incidence of severe reproductive tract complications associated with diagnosed genital chlamydial infection: the Uppsala Women's Cohort Study. Sex Transm Infect. 2006;82(3):212-8.

24. Dehghan Marvast L, Aflatoonian A, Talebi AR, Eley A, Pacey AA. Relationship between Chlamydia trachomatis and Mycoplasma genitalium infection and pregnancy rate and outcome in Iranian infertile couples. Andrologia. 2017;49(9).

25. Steiner RJ, Liddon N, Swartzendruber AL, Pazol K, Sales JM. Moving the Message Beyond the Methods: Toward Integration of Unintended Pregnancy and Sexually Transmitted Infection/ HIV Prevention. Am J Prev Med. 2018;54(3):4403.

26. EU Ar 2020;Pages. Accessed at https://abortreport.eu/netherlands/.

27. Coppus SF, Land JA, Opmeer BC, et al. Chlamydia trachomatis IgG seropositivity is associated with lower natural conception rates in ovulatory subfertile women without visible tubal pathology. Hum Reprod. 2011;26(11):3061-7.

28. Slurink IALVA, F.; Op de Coul, E.L.M.; Heijne, J.C.M.; van Wees, D.A.; Hoenderboom, B.M.; Visser, M;, den Daas, C.; Woestenberg, P.J.; Götz, H.M.; Nielen, M.; van Sighem, A.l.; Benthem, B.H.B Sexually transmitted infections in the Netherlands in 2018. 2019.

29. Hocking JS, Temple-Smith M, Guy R, et al. Population effectiveness of opportunistic chlamydia testing in primary care in Australia: a cluster-randomised controlled trial. Lancet. 2018;392(10156):1413-22.

30. Skulstad SM, Igland J, Johannessen A, et al. Validation of maternal reported pregnancy and birth characteristics against the Medical Birth Registry of Norway. PLoS One. 2017;12(8):e0181794. 


\section{Supplementary files}

- Supplementary table 1. Differences in pregnancy rate since sexual debut and time to pregnancy between chlamydia negative, positive and positive only by serology women.

- Supplementary Table 2. Differences in pregnancy rate since sexual debut and time to pregnancy between chlamydia negative, positive by CSI-NAAT, positive by chlamydia $\lg G$ presences or positive by self-reported infection.

- Supplementary figure 1. Forest plot of various sensitivity analyses of the risk for pregnancy following sexual debut between chlamydia positive and chlamydia negative women in hazard ratio's and $95 \% \mathrm{Cl}$.

- Supplementary figure 2 Forest plot of various sensitivity analyses of the chance for a pregnancy between chlamydia positive and chlamydia negative women with a pregnancy wish in hazard ratio's and $95 \% \mathrm{Cl}$. 
Supplementary Table 1. Differences in pregnancy rate since sexual debut and time to pregnancy between chlamydia negative, positive and positive only by serology women

Adjusted Hazard Ratio

Pregnancies

Chlamydia negative 1

Chlamydia positive 1.52

Chlamydia seropositive only $\quad 1.6$

$95 \% \mathrm{Cl}$

p-value

2. Years following sexual debut 7-10
Chlamydia negative
Chlamydia positive
1
1.48
$1.21-1.73$
$<0.01$
Chlamydia seropositive only
1.08
$0.69-1.67$
0.75

3. Years following sexual debut 11-13

Chlamydia negative 1

Chlamydia positive $\quad 0.95$

Chlamydia seropositive only $\quad 0.83$

$\begin{array}{lll}0.95 & 0.81-1.10 & 0.49 \\ 0.83 & 0.59-1.18 & 0.31\end{array}$

4. Years following sexual debut 13-23

Chlamydia negative

Chlamydia positive

1

Chlamydia seropositive only

$\begin{array}{lll}1.02 & 0.92-1.13 & 0.72\end{array}$

0.88

$0.69-1.11$

0.28

\section{Time to pregnancy}

\section{Age $16-29$ years}

Chlamydia negative

1

Chlamydia positive

0.77

0.65-0.91

$<0.01$

Chlamydia seropositive only

1.16

$0.69-1.94$

0.57

\section{Age 30-32 years}

Chlamydia negative

Chlamydia positive

1

Chlamydia seropositive only

0.76

0.60-0.95

0.02

0.67

$0.41-1.10$

0.11

\section{Age 33-39 years}

Chlamydia negative

Chlamydia positive

1

Chlamydia seropositive only

1.10

0.90-1.33

0.36

0.93

$0.59-1.44$

0.73

Chlamydia positive was defined as a positive NAAT-test outcome in the CSI study (CSI-NAAT), the presence of chlamydia IgG or a self-reported chlamydia infection. Chlamydia seropositive only was defined as being positive for chlamydia IgG, but not by self-report or by NAAT test outcome in the CSI study. For these analyses, multiple imputations were used to estimate time of first chlamydia infection in women without a known first year of chlamydia infection. Models 1-4 were adjusted for age, education level, migration background and number of lifetime partners. Models 5-7 were adjusted for age at first planned pregnancy/ age at trying to become pregnant and migration background. $\mathrm{HR}=$ Hazard Ratio. $\mathrm{Cl}=$ Confidence Interval. aHR = adjusted Hazard Ratio. 
Supplementary Table 2. Differences in pregnancy rate since sexual debut and time to pregnancy between chlamydia negative, positive by CSI-NAAT, positive by chlamydia IgG presences or positive by self-reported infection.

\begin{tabular}{|c|c|c|c|c|c|c|c|}
\hline \multirow[b]{2}{*}{ Pregnancies } & \multicolumn{3}{|c|}{ Adjusted Hazard Ratio } & & \multicolumn{3}{|c|}{ Adjusted Hazard Ratio } \\
\hline & $\mathrm{aHR}$ & $95 \% \mathrm{Cl}$ & P-value & & $\mathrm{aHR}$ & $95 \% \mathrm{Cl}$ & P-value \\
\hline \multicolumn{8}{|c|}{ Years following sexual debut $0-6$} \\
\hline Chlamydia negative & 1 & & & Chlamydia negative & 1 & & \\
\hline \multirow[t]{3}{*}{ Chlamydia positive } & 1.54 & $1.26-1.89$ & $<0.01$ & CSI-NAAT+ & 1.36 & $0.99-1.87$ & 0.06 \\
\hline & & & & $C t \lg G+$ & 1.90 & $1.23-2.93$ & $<0.01$ \\
\hline & & & & Self-reported + & 1.50 & $1.17-1.91$ & $<0.01$ \\
\hline \multicolumn{8}{|c|}{ Years following sexual debut 7-10 } \\
\hline Chlamydia negative & 1 & & & Chlamydia negative & 1 & & \\
\hline \multirow[t]{3}{*}{ Chlamydia positive } & 1.42 & $1.22-1.66$ & $<0.01$ & CSI-NAAT+ & 1.21 & $0.92-1.60$ & 0.18 \\
\hline & & & & Ct lgG + & 1.44 & $1.10-1.90$ & $<0.01$ \\
\hline & & & & Self-reported + & 1.52 & $1.26-1.84$ & $<0.01$ \\
\hline \multicolumn{8}{|c|}{ Years following sexual debut $11-13$} \\
\hline Chlamydia negative & 1 & & & Chlamydia negative & 1 & & \\
\hline \multirow[t]{3}{*}{ Chlamydia positive } & 0.93 & $0.80-1.07$ & 0.32 & CSI-NAAT + & 0.99 & $0.76-1.29$ & 0.94 \\
\hline & & & & $\mathrm{Ct} \lg \mathrm{G}+$ & 0.88 & $0.69-1.13$ & 0.32 \\
\hline & & & & Self-reported + & 0.93 & $0.77-1.13$ & 0.48 \\
\hline \multicolumn{8}{|c|}{ Years following sexual debut $14-23$} \\
\hline Chlamydia negative & 1 & & & Chlamydia negative & 1 & & \\
\hline \multirow[t]{3}{*}{ Chlamydia positive } & 1.00 & $0.90-1.10$ & 0.96 & CSI-NAAT+ & 0.95 & $0.76-1.18$ & 0.64 \\
\hline & & & & $\mathrm{Ct} \lg \mathrm{G}+$ & 0.81 & $0.68-0.96$ & 0.01 \\
\hline & & & & Self-reported + & 1.13 & $1.00-1.28$ & 0.05 \\
\hline \multicolumn{8}{|l|}{ Time to pregnancy } \\
\hline \multicolumn{8}{|l|}{ Age $16-29$ years } \\
\hline Chlamydia negative & 1 & & & Chlamydia negative & 1 & & \\
\hline \multirow[t]{3}{*}{ Chlamydia positive } & 0.79 & $0.67-0.94$ & $<0.01$ & CSI-NAAT + & 0.64 & $0.47-0.88$ & $<0.01$ \\
\hline & & & & Ct lgG + & 0.82 & $0.61-1.11$ & 0.21 \\
\hline & & & & Self-reported + & 0.87 & $0.70-1.08$ & 0.21 \\
\hline \multicolumn{8}{|l|}{ Age $30-32$ years } \\
\hline Chlamydia negative & 1 & & & Chlamydia negative & 1 & & \\
\hline \multirow[t]{3}{*}{ Chlamydia positive } & 0.74 & $0.60-0.92$ & $<0.01$ & CSI-NAAT + & 0.86 & $0.55-1.35$ & 0.51 \\
\hline & & & & Ct lgG + & 0.71 & $0.48-1.04$ & 0.08 \\
\hline & & & & Self-reported + & 0.73 & $0.55-0.95$ & 0.02 \\
\hline \multicolumn{8}{|l|}{ Age $33-39$ years } \\
\hline Chlamydia negative & 1 & & & Chlamydia negative & 1 & & \\
\hline \multirow[t]{3}{*}{ Chlamydia positive } & 1.07 & $0.89-1.28$ & 0.49 & CSI-NAAT+ & 1.32 & $0.82-2.12$ & 0.26 \\
\hline & & & & Ct lgG + & 0.88 & $0.64-1.22$ & 0.45 \\
\hline & & & & Self-reported + & 1.13 & $0.91-1.42$ & 0.27 \\
\hline
\end{tabular}

CSI-NAAT+ (positive) was defined as a positive NAAT-test outcome in the CSI study (CSI-NAAT) irrespectively of other positive chlamydia outcomes. Ct IgG + (Chlamydia trachomatis - Immunoglobulin G positive) was defined as the presence of chlamydia IgG, unless a participant was already positive by CSINAAT. Self-reported chlamydia positive was defined as a self-reported infection without a positive CSI-NAAT or presence of chlamydia IgG. For these analyses, multiple imputations were used to estimate time of first chlamydia infection in women without a known first year of chlamydia infection. Pregnancy models (first four) were adjusted for age, education level, migration background and number of lifetime partners. Time to pregnancy models (last three) were adjusted for age at first planned pregnancy/age at trying to become pregnant and migration background. $\mathrm{HR}=$ Hazard Ratio. $\mathrm{Cl}=$ Confidence Interval. aHR = adjusted Hazard Ratio. 


\section{Sensitivity analyses overall pregnancy rates}

$\operatorname{aHR}(95 \% \mathrm{Cl})$

Exposure time 0-6 year

Main analysis

Only second questionnaire women included

Analysis incl. only CSI-PCR positive versus negative

Pregnancy and $\mathrm{Ct}$ in same year excluded

Without multiple imputation

Exposure time 7-10 year

Main analysis

Only second questionnaire women included

Analysis incl. only CSI-PCR positive versus negative

Pregnancy and $\mathrm{Ct}$ in same year excluded

Without multiple imputation

Exposure time 11-13 year

Main analysis

Only second questionnaire women included

Analysis incl. only CSI-PCR positive versus negative

Pregnancy and $\mathrm{Ct}$ in same year excluded

Without multiple imputation

Exposure time 14-23 year

main analysis

only second questionnaire women included

Analysis incl. only CSI-PCR positive versus negative

Pregnancy and $\mathrm{Ct}$ in same year excluded

Without multiple imputation
$1.54(1.26,1.80)$

$1.81(1.37,2.39)$

$1.41(0.99,1.99)$

$1.29(1.04,1.60)$

$1.56(1.28,1.91)$

$1.51(1.36,1.67)$

$1.42(1.22,1.66)$

$1.36(1.11,1.66)$

$1.26(0.95,1.68)$

$1.37(1.17,1.60)$

$1.49(1.27,1.75)$

$1.40(1.29,1.52)$

$0.93(0.80,1.07)$

$0.94(0.78,1.14)$

$0.96(0.73,1.25)$

$0.88(0.76,1.02)$

$0.95(0.82,1.11)$

$0.93(0.86,1.00)$

$1.00(0.90,1.10)$

$0.99(0.88,1.11)$

$0.96(0.77,1.19)$

$0.99(0.89,1.09)$

$1.03(0.93,1.14)$

$1.00(0.95,1.05)$

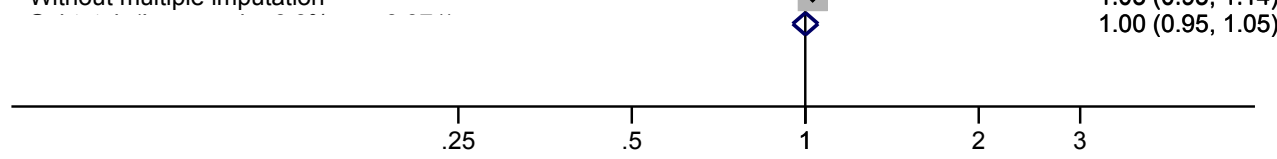

Adjusted Hazard Ratio's

Supplementary figure 1. Forest plot of various sensitivity analyses of the risk for pregnancy following sexual debut between chlamydia positive and chlamydia negative women in hazard ratio's and $95 \% \mathrm{Cl}$. Diamond shape $=$ overall aHR aHR $=$ adjusted hazard ratio $. \mathrm{Cl}=$ confidence interval. 


\section{Sensitivity analyses women with a pregnancy intention}

$\operatorname{aHR}(95 \% \mathrm{Cl})$

\section{Age group 16-29}

Main analysis

Only second questionnaire women included

Analysis incl. only CSI-PCR positive versus negative

Pregnancy and $\mathrm{Ct}$ in same year excluded

Without multiple imputation

Chlamydia positive after first pregnancy excluded

Age group 30-32

Main analysis

Only second questionnaire women included

Analysis incl. only CSI-PCR positive versus negative

Pregnancy and $\mathrm{Ct}$ in same year excluded

Without multiple imputation

Chlamydia positive after first pregnancy excluded

Age group 33-39

Main analysis

Only second questionnaire women included

Analysis incl. only CSI-PCR positive versus negative

Pregnancy and $\mathrm{Ct}$ in same year excluded

Without multiple imputation

Chlamydia positive after first pregnancy excluded

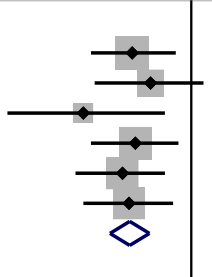

$0.79(0.67,0.94)$

$0.85(0.68,1.05)$

$0.65(0.48,0.90)$

$0.80(0.67,0.95)$

$0.76(0.63,0.90)$

$0.78(0.65,0.93)$

$0.78(0.72,0.85)$

$0.74(0.60,0.92)$

$0.71(0.56,0.90)$

$0.85(0.54,1.34)$

$0.73(0.58,0.91)$

$0.73(0.58,0.92)$

$0.73(0.58,0.92)$

$0.73(0.66,0.81)$

$1.07(0.89,1.28)$

$1.05(0.87,1.28)$

$1.26(0.79,2.01)$

$1.07(0.88,1.29)$

$1.01(0.83,1.23)$

$1.09(0.90,1.33)$

$1.06(0.98,1.16)$

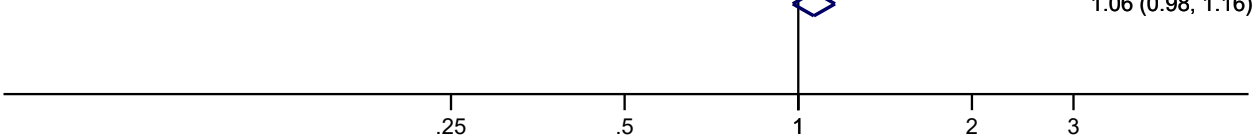

\section{Adjusted Hazard Ratio's}

Supplementary figure 2 Forest plot of various sensitivity analyses of the chance for a pregnancy between chlamydia positive and chlamydia negative women with a pregnancy wish in hazard ratio's and $95 \% \mathrm{Cl}$. Diamond shape $=$ overall aHR. aHR $=$ hazard ratio. $\mathrm{Cl}=$ confidence interval. 



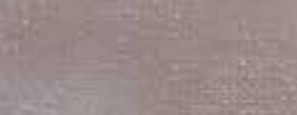

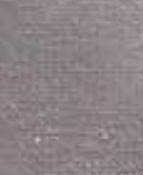

\section{sisting}

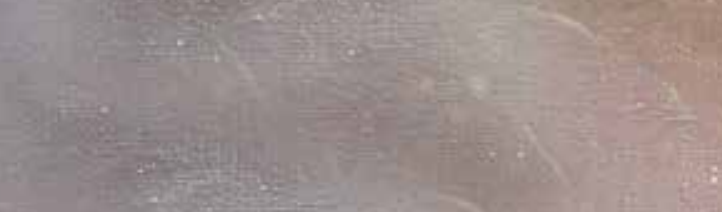

जince

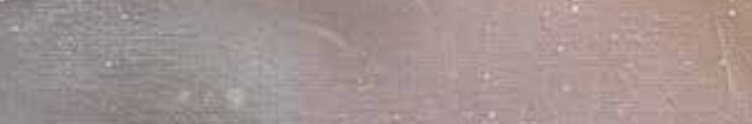

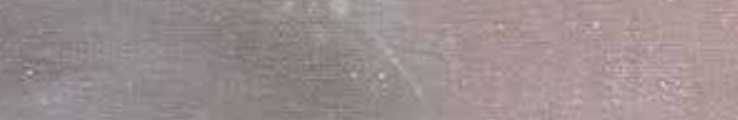

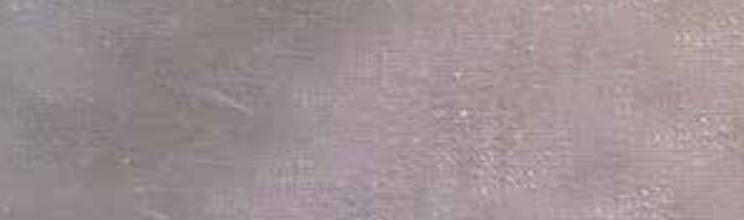

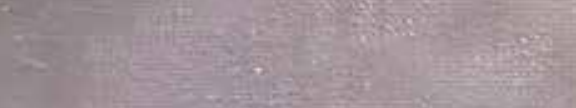

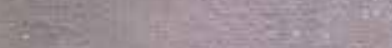

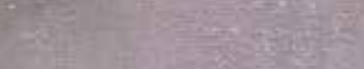

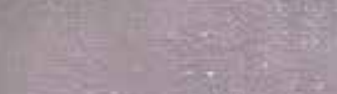

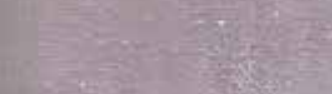

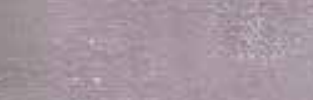

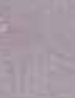

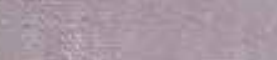

netwo

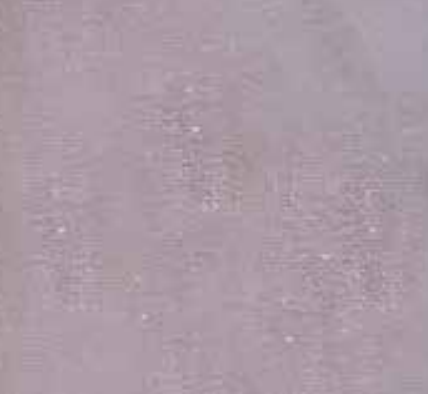

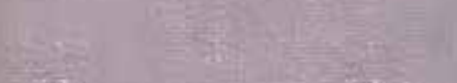

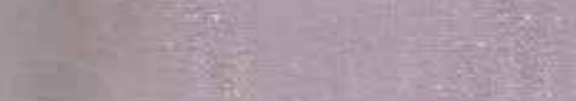


9

General discussion 


\section{General discussion}

Chlamydia trachomatis (chlamydia) natural history has been studied for decades, but questions remain and challenges in studying the natural history of chlamydia are not easily resolved.

This thesis describes research about the risk of, and related risk factors for, Chlamydia trachomatis related complications studied in a longitudinal cohort study of women of reproductive age. Advantages of this cohort construction, challenges in studying the natural history, and the validation of the followed methods were described. Chlamydia serology in its use either in determining previous chlamydia infections as well as a potential predictive factor for related complications were examined. The risk for pelvic inflammatory disease (PID), ectopic pregnancy and tubal factor infertility (TFI) following a chlamydia infection was determined as also chances for pregnancy and time to pregnancy in women with and without a previous chlamydia infection. In this discussion, the most important findings of this thesis will be elaborated, discussed and future perspectives will be explored in the following order:

1. Lessons learned from the Netherlands Chlamydia Cohort set up

2. The value of Chlamydia trachomatis serology

3. Long-term complications of chlamydia infections and predictive factors

4. Chlamydia control: The way forward

\section{Lessons learned from the Netherlands Chlamydia Cohort set up}

In the Dutch national action plan for STIs, HIV and sexual health it was proposed to develop a new strategy for chlamydia control to reduce the burden of chlamydia infection(1). Chlamydia prevalence reduction is not feasible, but reduction of chlamydia sequelae might be. To gain insight in the burden of chlamydia infections and to estimate risks and risk factors for chlamydia related complications the Netherlands Chlamydia Cohort Study (NECCST) was set up to guide policy making in a changing mentality of chlamydia control. To prevent publication bias and to maximize insight in the used protocol, the study protocol was published (Chapter 2) (2, 3). This study not only yielded relevant results, but strengths and limitations from the methodology itself can aid future research(4). The most relevant strengths and limitations will be discussed.

\section{Building on a previous cohort}

NECCST has a longitudinal study design in which a combination of retrospective and prospective data was used. This design allows us to accurately study temporality in chlamydia infections and multiple late chlamydia complication outcomes(5). The cohort builds on the previously executed Chlamydia Screening Implementation (CSI) study. The CSI, a population based screening study, included $>300.000$ men and women. Although women could only be invited for NECCST if they consented to be approached again for future STI research, this still comprised of about 15,000 women. These women were followed from 2008 onwards, resulting in a long follow-up. While building on the CSI ensured long follow-up, relevant data, and samples which could be reused, the prospective part of NECCST enabled the possibility to collect additional biological samples 
and to collect specific data over time needed to answer the research questions. The latter is a major advantage over registered based studies in which data is already collected and therefore not specific for the research target(5-7). Although properly conducted randomized controlled trials (RCTs) have the highest level of evidence, RCTs are for evaluating an intervention not for evaluating a natural course of an infection(8). Furthermore, RCTs are not a preferred design when long-follow up is needed. This makes it difficult to study chlamydia disease outcomes (specifically ectopic pregnancies and tubal factor infertility [TFI]). For example, a previous well-designed RCT that studied the intervention of chlamydia screening on PID outcome had only one year of follow-up and failed to find statistical significant results due to insufficient sample size(9).

Although prospective cohort studies can be too time-consuming and costly, building on the CSI allowed us to study chlamydia disease outcomes in this observational cohort study design. Despite major advantages from this design, biases should be carefully addressed and considered in drawing conclusions and generalizing the results.

\section{Defining chlamydia status using multiple parameters}

A major challenge in preventing bias in the exposure outcome is misclassification in chlamydia status. This misclassification arises due to the asymptomatic nature of the infection. To reduce misclassifications three parameters were used to determine previous infection (Figure 1), although all with its own limitations. NAAT tests only detect current infections and infections in the lower genital tract. Serology detects previous infection, but with imperfect test sensitivity, and selfreported infections are prone to recall bias. However, in absence of a perfect serology test and the ability to test everybody monthly, combining these three outcomes will results in the least misclassifications possible in chlamydia research.

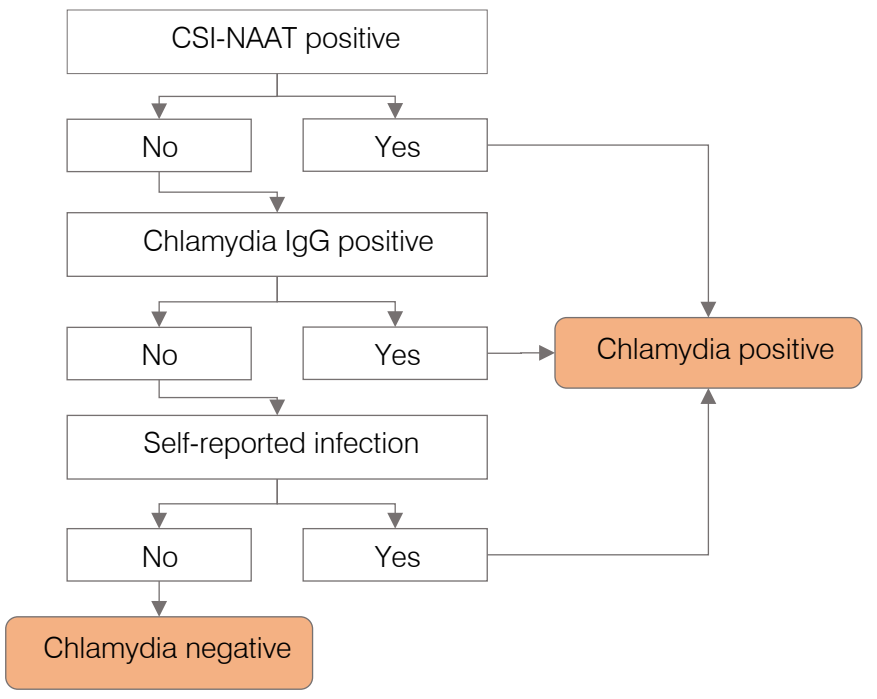

Figure 1. Decision tree for chlamydia status definition. CSI-NAAT = chlamydia screening implementation nucleic acid amplification test. IgG = immunoglobulin. 
Medical record checks for outcome variables

Self-reported outcomes were used for PID status, ectopic pregnancies, TFI, and (time to) pregnancy. Self-reporting is always prone to recall bias. But bias on pregnancy outcomes was expected to be limited. Validity of self-reported data concerning pregnancies from questionnaires was considered high in a study comparing questionnaire data and birth registry's in Norway. This was independent of the number of recall years (up to 45 years after birth)(10). For PID diagnosis this is more challenging. Due to lack of clear criteria for a PID diagnosis(11), occurrence of silent PIDs(12), and the risk for diagnostic bias(13), possible bias is difficult to prevent. To increase the validity on PID, incidences of PIDs among chlamydia positive and negative women found in NECCST were compared to other studies that determine PID with different methods. For the diagnosis of TFI, a validation in medical registers was carried out. All women who reported to be infertile were asked for permission to retrieve their medical files to validate their answers. Selfreported TFI was found to be a valid answer after comparison with medical files(14).

\section{Validation of methods for blood collections and extraction of DNA on stored samples}

It is essential to validate methods prior use as a (diagnostic) tool to test the accuracy and to assess if the method measures what is was intended to do(15). In NECCST, biological samples were collected for chlamydia antibody determination and retrieved for host DNA analyses. Capillary blood was home-collected by participants themselves, this method was tested using a selection of the NECCST cohort that yielded valid results before it was applied to the entire cohort (chapter 3). To reduce costs, old CSI samples (i.e. vaginal swabs and urine samples) were retrieved from the biobank and tested for host DNA. In a SNP pilot study the use of these old samples for DNA retrieval SNP determination was successful in over $90 \%$ of DNA samples (chapter 4).

In answering the research questions all strengths and limitations of this unique cohort that might result in biases were considered and taken into account. Answers to the research questions should help chlamydia control to be more effective, more efficient and goal oriented.

\section{The value of Chlamydia trachomatis serology}

Chlamydia serology might be one of the means that can help making chlamydia control more effective, efficient and targeted(16). Nowadays chlamydia serology has four key attributes: 1) Chlamydia surveillance (17), 2) Measuring population attributable fraction of chlamydia on complications (18), 3) Vaccine development \& evaluation(19) and 4) Identify antigens/combinations of antigens that identify women with chlamydia related complications(20). Regarding the use of serology for chlamydia surveillance, testing for chlamydia antibodies in a specific population can identify how many persons have been ever exposed to chlamydia. Seroprevalence between different age-groups and birth cohorts can be compared to gain insight into cumulative risk of exposure and contribute to monitoring frequency and control of chlamydia(17). Using serology to monitor chlamydia seroprevalence is probably more effective in women compared to men, because chlamydia antibodies assays seem to have a higher sensitivity in women(21). But also in women sensitivity and specificity are not optimal. Not all women develop antibodies against 
specific antigens and antibodies can wane over time. In Chapter 5 the value of chlamydia MOMP antibody testing was evaluated within women from the NECCST study. Over 3,500 NECCSTparticipants were tested for chlamydia IgG antibodies using the commercially available CT IgG ELISA plus (Medac, Wedel, Germany). Test sensitivity was previously determined at $71 \%$ at time of infection(22). Seroprevalence was $41 \%$ among chlamydia positive women (previously chlamydia NAAT positive) and 8\% among chlamydia negative women (no positive NAAT test results). The specificity of the Medac test (although high-97\%) could, in combination with the low seroprevalence in the chlamydia negative group, result in a considerable number of false positives. However, the specificity issues reported in general in the past based on the potential cross reactivity with Chlamydia pneumoniae, Chlamydia psittaci, and Acinetobacter were not applicable in the current peptide-based ELISA from Medac in which a small peptide from the MOMP protein was selected with no cross reactivity with $C$. pneumoniae, in contrast to either lipopolysaccharide-based ELISAs or total MOMP-based ones(23-25). When outcomes of selfreported positives tests, chlamydia NAAT tests and chlamydia serology were taken together, the combined chlamydia positivity increased from $23 \%$ to $29 \%$. The seroprevalence of forty percent among women previously tested positive for chlamydia is comparable to the $38 \%$ and $35 \%$ found in other studies determining chlamydia antibodies using the Medac MOMP assay years following infection(26, 27). The eight percent seropositives among previously negative tested women were infections otherwise missed. These women were than misclassified as chlamydia negative. Reductions in misclassifications increase the precision of estimates on proportions of women who experience chlamydia related complications. Because of this it is recommended to use serology in addition to other chlamydia measures such as previous NAAT results and possible self-reported infections.

However, other assays for example the EB ELISA and the Pgp3 ELISA reported higher sensitivities and specificities than the Medac assasy for estimating chlamydia population seroprevalence(21, 28). The Medac assay uses the MOMP antigen as a target. MOMP is a large protein involved in maintaining rigidity of the chlamydia membrane, attachment to human epithelial cell and that functions as a pore to provide nutrients once the bacterium entered the host cell $(29,30)$. Nevertheless, not everyone will develop antibodies against this specific antigen. This will probably be similar with other one-target assays. Chlamydia trachomatis consist of 895 open reading frames. In theory once infected the host immune response can develop antibodies against all of these 895 antigens. In Chapter 6 the development and use of a Chlamydia trachomatis whole proteome array was described. The aim was to identify antigens best in distinguishing between chlamydia infected and non-infected and between women with and without chlamydia related complications using all 895 open reading frames(31). A panel of in total eighteen antigens could be constructed to identify $96 \%$ of all seropositives instead of using all antigens. A panel of eighteen different antigens can easily be used in multiplex assays similar to for example an HPV assay with seventeen antigens(32). Starting from including all 895 antigens to finally include less than two dozen antigens most reactive is an elegant method to detect the best suitable and promising antigens. In the panel several well-known and used antigens were validated such as 
CPAF, MOMP, Pgp3, TARP and HSp60 (17, 20), but also antigens not previously identified as targets or even described were found such as CT_142. Validation of this panel is however vital before it can be used as an assay to identify previous infections. These antigens were most reactive among NECCST participants, which might not be comparable to all women of reproductive age. However, the seven most reactive antigens were also identified as highly immunogenic when analyzing samples from the Mongolian population based study on chlamydia whole proteome arrays(31). Nevertheless, validation in different cohorts and preferably in cohort without any Chlamydia trachomatis exposure but with Chlamydia pneumoniae exposure is needed to rule out cross-reactivity. Due to a high seroprevalence among previously tested chlamydia negative women (although with chlamydia related complications) cross-reactivity with the whole proteome array with other chlamydia species cannot be excluded.

A second objective was to explore disease specific markers. The sample size of women with a complication, specifically ectopic pregnancy and TFI unfortunately was too low to detect any differences in prevalence. However, CT_142 and CT_813 were less common among PID cases compared to controls and CT_858 was less common among women with chronic pelvic pain compared to controls. Larger sample sizes are needed to further explore these associations.

Chlamydia serology can aid to the surveillance, evaluation of chlamydia control activities and identification of disease specific risk. Current assays however lack sensitivity and specificity in determining population seroprevalence. This diminishes the value of chlamydia serology for this purpose. Assays with improved sensitivity and specificity are needed. Whole proteome arrays are useful in determining important potential antigens for inclusion in assays. Additionally, identifying disease specific markers will give the opportunity to identify women most at risk for complications. In NECCST using chlamydia MOMP-serology reduced to a certain extent misclassification of chlamydia status, but did not remove it completely.

\section{Long-term complications of chlamydia infections and predictive factors}

Pelvic inflammatory disease, ectopic pregnancy and tubal factor infertility are major severe complications following a (asymptomatic) chlamydia infection. The risk of developing one or more of these complications is determinative for chlamydia control activities(33). It is therefore vital to have decent risk estimates of late complications following an infection. In the first chlamydia natural history studies risk estimates following infections were high. For PID, either clinical or subclinical, the risk following chlamydia infection was estimated between 25 to $80 \%(34-43)$. The risk of ectopic pregnancy following a PID was estimated between 5-25\%(37, 44-48) and TFI was assumed to occur among 10 to $20 \%$ of all women with chlamydia-related $\operatorname{PID}(44,45,49-55)$. In recent studies, risk estimates for late complications are much lower. In a multi-parameter evidence synthesis published in 2016 risk estimates for PID (symptomatic and asymptomatic) following untreated chlamydia infection was $17 \%$, for ectopic pregnancy this was $0.2 \%$ and for TFI 0.5\%(18). Explanations for the differences in risk between older studies and recent studies might be that older studies included high risk STI populations, in high risk STI populations multiple chlamydia infections are more common and the risk for other STI such as Gonorrhea, which is 
also an etiological factor for PID, is also higher(34). Furthermore, case-control studies were used to estimate absolute risk, which is not a suitable design, and last some studies assumed that all ectopic pregnancies were preceded by PID(33). These factors might have overestimated risks for chlamydia complications. In Chapter 7 risks for PID, ectopic pregnancy and TFI were estimated in the NECCST cohort. The NECCST cohort includes 5700 women between the age of 21 and 38 following the first round. Three in ten women were classified as chlamydia positive based on CSI-NAAT tests, chlamydia serology, or self-reported chlamydia infections. Incidence rates for PID were considerably higher among chlamydia positive versus negative women, 4.4 per 1000 person-years (py) and 1.4/1000py respectively. For TFI this was 1.3/1000py for positives and $0.2 / 1000$ py for negatives. No difference was seen in the incidence rate of ectopic pregnancies, 0.8/1000py for chlamydia positives and 0.6/1000py for negatives. These risk estimates were rather comparable to other more recent studies on chlamydia related complications. Population based cohort studies from the UK, Denmark and Australia found risk differences and incidence rates for PID in chlamydia positive women of 4.0-8.0/1000py, 2.8/1000py for ectopic pregnancy and $0.7 / 1000$ py for $\operatorname{TFI}(6,7,56,57)$. Given the rather different study design of the NECCST study and the population based cohort studies, it is very interesting and reassuring that risk estimates were comparable. This increases the certainty of yielded results.

Although all these studies showed an increased risk for PID and TFI following chlamydia infection, this does not automatically support chlamydia screening or testing/treating of risk groups to prevent complications. In NECCST, as well as in the population based studies, higher risks between 30-100\% for PID were found in women who were almost all treated for their infection compared to women tested negative for a chlamydia infection. Why does treatment not prevent these women from PID? Is treatment given to late? Moreover, women in NECCST who were tested positive for chlamydia only by a serology test and did therefore presumably not get treated for their infection, had similar risk estimates for complications as women who were treated. If treatment in asymptomatic women would reduce the risk for complications, one would have expected higher risks among serology positives only. In addition to this, overall risk estimates were small. Cumulative risk estimates in NECCST as well as in other studies ranged between $2-5 \%$ for PID, $0.7-3 \%$ for ectopic pregnancy and $0.6-1.1 \%$ for $\operatorname{TFI}(6,7,56)$. Low incidence rates challenge the cost-effectiveness of chlamydia screening programs(58-60). Because the lower the overall risk for PID, ectopic pregnancy and TFI, the higher the number needed to screen to prevent a case(61). Instead of taking TFI as an outcome, one can also focus on pregnancy rates. In Chapter $\mathbf{8}$ pregnancy rates and time to pregnancy was compared between chlamydia positive and negative women. Overall pregnancy rates, either planned an unplanned, were rather similar in the two groups. In the first ten years following sexual debut, even more chlamydia positive women became pregnant. However, among women with a pregnancy wish, time to pregnancy was significantly longer among chlamydia positive women compared to negative women; twelve versus eight percent had a time to pregnancy of over twelve months. These results were difficult to interpret. Chlamydia positive women had significantly more often unplanned pregnancies compared to negative women. Although this is not unexpected since unplanned pregnancies and chlamydia infection are both related to unprotected sexual contact(62), this could have introduced 
selection bias in time to pregnancy analyses. In time to pregnancy analyses only women who were planning to become pregnant were included, because of the need for a fixed 'time to pregnancy', which is absent in case of an unplanned pregnancy. Since more chlamydia positive women were excluded, whom were al proven fertile, it is difficult to conclude that the longer time to pregnancy is associated with the chlamydia infection or that the found effect is due to selection bias. Only few other studies determined pregnancy rates, birth rates, or time to pregnancy in chlamydia positive and negative women. Two large population based register studies from Denmark and Norway in 2005 and 2007 indicated no difference in birth rates following a positive or negative chlamydia test result $(63,64)$. In a study among infertile women in whom no specific cause of infertility could be determined, time to pregnancy was longer among women with chlamydial antibodies compared to women without(65). All pregnancy related results taken together might indicate that the effect of chlamydia on infertility is too small to be found in large population based studies. Other factors such as sexual risk behavior and sexual frequency might have a larger impact. In specific groups time to pregnancy was found to be longer among chlamydia positive women. This might indicate that infertility and longer time to pregnancy is only found in a small proportion of women following a chlamydia infection.

Taking all chlamydia outcomes into account, i.e. PID, ectopic pregnancy, TFI, overall pregnancies and time to pregnancies, it can be concluded from our data as well as previous studies that previous chlamydia infections does increase the risk for some reproductive tract outcomes. Overall complications rates were however low and pregnancy rates high. Only few women suffer from late chlamydia complications. Why is the course of infection different between these women and women without complications? How then to address the chlamydia burden? Reinfection rates, sexual risk behavior, bacterial factors, and host genetics all play an important role in the course of infection(6, 66-69). In the NECCST cohort women who had a symptomatic infection were more at risk for a PID compared to women with an asymptomatic infection. TFI was more often diagnosed among women who had a young age at first chlamydia infection ( $<20$ years of age). Both suggest that a different immunological response might be an explanation for the increased risk. Young age is a consistent risk factor for chlamydia infection and PID, despite lower sexual activity compared to women of older age, which implies that host immunity is at work $(66,70)$. Host immunity and genetics might be key in differentiating between women with and without (risk for) chlamydia related complications(71). In the pilot SNP study (chapter 4) a first exploration as predicters for developing chlamydia complications was done for four SNPS (TLR2 +2477 $\mathrm{G}>\mathrm{A}$, NOD1 + $32656 \mathrm{~T}->\mathrm{GG}, \mathrm{CXCR5}+10950 \mathrm{~T}>\mathrm{C}$, and IL10 -1082 A > G) previously associated with chlamydia disease outcomes. We found that NOD1 + 32656 T->GG increased the risk for a symptomatic (versus asymptomatic) chlamydia infection and IL10 -1082 A>G increased the risk for late chlamydia complications. When having enough associated factors, a predictive tool can be built to identify women with high risk profiles for late chlamydia complications. Preventive measures can be targeted on those women. 


\section{Chlamydia control: The way forward}

In the Netherlands extensive chlamydia control efforts are ongoing. Risk groups, such as people with symptoms, people notified for a chlamydia infection, and youngsters until the age of 25 can be tested and treated for chlamydia free of charge(72). In 2018/2019 in the Netherlands, over 150,000 tests were performed at the STI clinics and over 300,000 STI consultations (either infection of fear of STI) were performed by general practitioners. About 60,000 people tested positive and were treated accordingly(72). These numbers increase yearly. Evidence of a decline in chlamydia prevalence due to screening is lacking as seen in many national and international studies $(21,59,73,74)$. The effect of screening on late complications seems marginal, maybe test and treatment was too late to prevent complications $(9,73)$. Additionally, complication rates as seen in the NECCST cohort were low as well as the estimated attributable fractions of chlamydia of PID, ectopic pregnancy and TFI(18). A decline of PID and ectopic pregnancy cases since the implementation of chlamydia control efforts was seen in many countries, however the decline was similar in countries with extensive chlamydia control efforts as well as in countries without(11). In a natural experiment in Sweden where many chlamydia infections were missed (and not treated), because of a chlamydia variant which was not picked up by diagnostics, complications rates were only marginally effected(75). And last in the NECCST cohort women who tested positive by serology only (and were presumably not treated) had similar complication rates as chlamydia positive women who were treated. Advantages of screening or testing and treating risk groups seem (highly) overestimated.

What about disadvantages of current practices, the so called benefit-harm ratio? The goals of screening are to detect and treat asymptomatic infections, to limit ongoing transmission, and to reduce the incidence of sequelae. With early detection and treatment of asymptomatic infections the duration of infection is shortened and transmission will be reduced(59). But shortening the duration of the infection might have an adverse effect. Early antimicrobial treatment might interfere with the development of natural immunity induced by the infection, this is called the arrested immune hypothesis $(76,77)$. That would mean that after treatment people are fully susceptible again for re-infection. Re-infections increase the risk for late complications(6). Population susceptibility is then enhanced and after implementing control programs, chlamydia prevalence will more likely increase than decrease(76). A second adverse effect is that being diagnosed with a chlamydia infection or even just taking a chlamydia test is associated with stigma. In a Dutch study, youngsters felt ashamed to have 'caught' an infection and might think they should have better taken care of themselves(78). A more recent concern is overtreatment. Testing and treatment to reduce transmission is no longer substantiated given the absence of a prevalence reduction. Treatment is than focused on the prevention of late complications. But complications rates are, fortunately, very low. Therefore, many women and specially men are treated unnecessary. If only one percent of women progresses to TFI, $99 \%$ of women will be treated while the infection would have spontaneously cleared without causing TFI. Within one year already fifty percent of women will have cleared the infection(79). The chlamydia bacterium itself remains sensitive to first line antibiotics(80), but other pathogens such as MG and NG may become resistant due to overtreatment. 
The time has come to change chlamydia control practices in the Netherlands. Testing almost half a million people and treating over 60,000 persons annually without solid evidence that this result in transmission reduction or in preventing late complications is no longer justified. In November 2019, the Dutch Chlamydia Top was organized driven by the first results of the NECCST cohort and other recent literature on chlamydia complications and control activities. Dutch experts as well as several international chlamydia experts were invited to discuss the current chlamydia paradigm and how to move forward. At time of writing this thesis a viewpoint of the meeting is being written. The focus of chlamydia control programs should be on the $5 \%$ of women who will develop serious sequelae. In the absence of vaccines, the ultimate measure will be to have an prognostic tool which differentiates between women with and without an increased risk for late complications. This tool, will be based on genetic markers, immunologic markers, but also risk behavior and health characteristics. A subset of 25 SNPs that was previously linked to or biologically plausible to be related to the development of complications following a chlamydia infection, was determined on DNA samples from NECCST participants. Using statistical analyses on the ongoing NECCST cohort, SNPs with the highest predictive value will be implemented in the diagnostic tool. This tool can be implemented in STI clinics, in fertility clinics or maybe even in GP practices. In STI clinics and GP practices, this tool might differentiate between women who need antibiotics and who don't. Additionally the tool can aid the follow-up of high risk profile women. Treatment has to be effective and re-infections should be prevented(6). In fertility clinics, these women can directly helped further in the fertility workup without examination and treatment delay. Until such a tool is in place, current test and treat practices can be more focused on risk groups. Until now not only women are being tested but also heterosexual men and men who have sex with men (MSM). Since transmission cannot be stopped and complications in men after asymptomatic infection are not likely, it might be an idea to stop testing men and test and treat only if the female partner tested positive. It is proposed that chlamydia infection in MSM might increase the acquisition of an HIV infection(81), but in light of all other HIV preventive measures such as $\operatorname{PrEP}(82)$ and treatment as prevention (TASP)(83) this became less relevant in countries with a low HIV incidence and undetectable viral load in people living with HIV. The risk for chlamydia as well as PID is highest among women $<20$ years $(66,72)$. They should have priority over people in their twenties. Additionally a PID risk score based on current evidence might be used to classify women as high or low risk for PID. Different options for more effective chlamydia control activities can be explored while in the meantime a prognostic tool based on data of the NECCST cohort will be developed.

NECCST, a unique longitudinal cohort investigating chlamydia disease progression gave insight in risks for late chlamydia complications and pregnancies. Challenges and limitations in this cohort were acknowledged and included in conclusion making. Current results and continuation of the cohort will guide policy making regarding chlamydia control. In 2020 a second ZonMw (The Netherlands Organisation for Health Research and Development) grant on the NECCST cohort was approved. This will result in longer follow-up of the NECCST participants, which results in more disease specific outcomes. Furthermore, to comprehend the complete burden of chlamydia infections on female reproductive health, outcomes on miscarriages, preterm birth, and still birth 
will be investigated. The final aim is to focus on identifying the target group of women whom benefit from chlamydia preventive measures by developing a prognostic tool. This tool will be piloted to test acceptability and feasibility. Ultimately, results of NECCST will aid to increase effectiveness, efficiency, and targeted health care of chlamydia control activities. 


\section{References}

1. David S, van Benthem, B., Deug, F., van Haastrecht, P. Nationaal Actieplan soa, hiv en seksuele gezondheid 2017-2022. Bilthoven: Rijksinstituut voor Volksgezondheid en Milieu (RIVM); 2018.

2. Summerskill $W$, Collingridge $D$, Frankish $H$. Protocols, probity, and publication. Lancet. 2009;373(9668):992.

3. Chan AW, Song F, Vickers A, Jefferson $T$, Dickersin K, Gotzsche PC, et al. Increasing value and reducing waste: addressing inaccessible research. Lancet. 2014;383(9913):257-66.

4. Vieira RF, , de Lima, R.C., Mizubuti, E.S.G. How to write the discussion section of a scientific article. Acta Scientiarum. 2018;41.

5. Setia MS. Methodology Series Module 1: Cohort Studies. Indian J Dermatol. 2016;61(1):21-5.

6. Davies B, Turner KME, Frølund M, Ward H, May MT, Rasmussen S, et al. Risk of reproductive complications following chlamydia testing: a population-based retrospective cohort study in Denmark. The Lancet Infectious Diseases. 2016;16(9):1057-64.

7. Low N, Egger M, Sterne JA, Harbord RM, Ibrahim F, Lindblom B, et al. Incidence of severe reproductive tract complications associated with diagnosed genital chlamydial infection: the Uppsala Women's Cohort Study. Sex Transm Infect. 2006;82(3):212-8.

8. Burns PB, Rohrich RJ, Chung KC. The levels of evidence and their role in evidencebased medicine. Plast Reconstr Surg. 2011;128(1):305-10.

9. Oakeshott P, Kerry S, Aghaizu A, Atherton $\mathrm{H}$, Hay S, Taylor-Robinson D, et al. Randomised controlled trial of screening for Chlamydia trachomatis to prevent pelvic inflammatory disease: the POPI (prevention of pelvic infection) trial. BMJ. 2010;340:c1642.

10. Skulstad SM, Igland J, Johannessen A, Bertelsen RJ, Lonnebotn $M$, Omenaas $\mathrm{ER}$, et al. Validation of maternal reported pregnancy and birth characteristics against the
Medical Birth Registry of Norway. PLoS One. 2017;12(8):e0181794.

11. Unemo M, Bradshaw CS, Hocking JS, de Vries HJC, Francis SC, Mabey D, et al. Sexually transmitted infections: challenges ahead. Lancet Infect Dis. 2017;17(8):e235-e79.

12. Brunham RC, Gottlieb SL, Paavonen J. Pelvic inflammatory disease. N Engl $J$ Med. 2015;372(21):2039-48.

13. 13. Haggerty CL, Gottlieb SL, Taylor BD, Low N, Xu F, Ness RB. Risk of sequelae after Chlamydia trachomatis genital infection in women. J Infect Dis. 2010;201 Suppl 2:S13455.

14. Hoenderboom BM, van Benthem BHB, van Bergen J, Dukers-Muijrers N, Gotz HM, Hoebe C, et al. Relation between Chlamydia trachomatis infection and pelvic inflammatory disease, ectopic pregnancy and tubal factor infertility in a Dutch cohort of women previously tested for chlamydia in a chlamydia screening trial. Sex Transm Infect. 2019;95(4):300-6.

15. Gold R, Reichman M, Greenberg E, Ivanidze $J$, Elias E, Tsiouris AJ, et al. Developing a new reference standard: is validation necessary? Acad Radiol. 2010;17(9):1079-82.

16. 16. Woodhall SC, Gorwitz RJ, Migchelsen SJ, Gottlieb SL, Horner PJ, Geisler WM, et al. Advancing the public health applications of Chlamydia trachomatis serology. Lancet Infect Dis. 2018;18(12):e399-e407.

17. Horner P, Soldan K, Vieira SM, Wills GS, Woodhall SC, Pebody R, et al. C. trachomatis Pgp3 antibody prevalence in young women in England, 1993-2010. PLoS One. 2013;8(8):e72001.

18. Price MJ, Ades AE, Soldan K, Welton NJ, Macleod J, Simms I, et al. The natural history of Chlamydia trachomatis infection in women: a multi-parameter evidence synthesis. Health Technol Assess. 2016;20(22):1-250.

19. de la Maza LM, Zhong G, Brunham RC. Update on Chlamydia trachomatis Vaccinology. Clin 
Vaccine Immunol. 2017;24(4).

20. van Ess EF, Eck-Hauer A, Land JA, Morre SA, Ouburg S. Combining individual Chlamydia trachomatis IgG antibodies MOMP, TARP CPAF, OMP2, and HSP60 for tubal factor infertility prediction. Am J Reprod Immunol. 2019;81(3):e13091.

21. Woodhall SC, Wills GS, Horner PJ, Craig R, Mindell JS, Murphy G, et al. Chlamydia trachomatis Pgp3 Antibody Population Seroprevalence before and during an Era of Widespread Opportunistic Chlamydi Screening in England (1994-2012). PLoS One. 2017;12(1):e0152810.

22. Morre SA, Munk C, Persson K, Kruger-Kjaer S, van Dijk R, Meijer CJLM, et al. Comparison of Three Commercially Available Peptide-Based Immunoglobulin $G(\lg G)$ and $\lg A$ Assays to Microimmunofluorescence Assay for Detection of Chlamydia trachomatis Antibodies. Journal of Clinical Microbiology. 2002;40(2):584-7.

23. Forsey T. Antibodies to Chlamydia trachomatis. Genitourin Med. 1987;63(4):279.

24. Brade H, Brunner H. Serological cross-reactions between Acinetobacter calcoaceticus and chlamydiae. J Clin Microbiol. 1979;10(6):81922.

25. Land JA, Gijsen AP, Kessels AG, Slobbe ME, Bruggeman CA. Performance of five serological chlamydia antibody tests in subfertile women. Hum Reprod. 2003;18(12):2621-7.

26. Horner PJ, Wills GS, Reynolds R, Johnson AM, Muir DA, Winston A, et al. Effect of time since exposure to Chlamydia trachomatis on chlamydia antibody detection in women: a cross-sectional study. Sex Transm Infect. 2013;89(5):398-403.

27. Ohman H, Rantsi T, Joki-Korpela P, Tiitinen A, Surcel HM. Prevalence and persistence of Chlamydia trachomatis-specific antibodies after occasional and recurrent infections. Sex Transm Infect. 2019.

28. Gupta K, Brown L, Bakshi RK, Press CG, Chi X, Gorwitz RJ, et al. Performance of Chlamydia trachomatis OmcB Enzyme-Linked
Immunosorbent Assay in Serodiagnosis of Chlamydia trachomatis Infection in Women. J Clin Microbiol. 2018;56(9)

29. Stephens RS, Sanchez-Pescador R, Wagar EA, Inouye C, Urdea MS. Diversity of Chlamydia trachomatis major outer membrane protein genes. J Bacteriol. 1987;169(9):3879-85.

30. Newhall WJt. Biosynthesis and disulfide crosslinking of outer membrane components during the growth cycle of Chlamydia trachomatis. Infect Immun. 1987;55(1):162-8.

31. Hufnagel $\mathrm{K}$, Lueong $\mathrm{S}$, Willhauck-Fleckenstein M, Hotz-Wagenblatt A, Miao B, Bauer A, et al. Immunoprofiling of Chlamydia trachomatis using whole-proteome microarrays generated by on-chip in situ expression. Sci Rep. 2018;8(1):7503

32. Waterboer T, Sehr P, Michael KM, Franceschi $\mathrm{S}$, Nieland JD, Joos TO, et al. Multiplex human papillomavirus serology based on in situ-purified glutathione s-transferase fusion proteins. Clin Chem. 2005;51(10):1845-53.

33. van Valkengoed IG, Morre SA, van den Brule AJ, Meijer CJ, Bouter LM, Boeke AJ. Overestimation of complication rates in evaluations of Chlamydia trachomatis screening programmes-implications for cost-effectiveness analyses. Int J Epidemiol. 2004;33(2):416-25.

34. Scholes D, Stergachis A, Heidrich FE, Andrilla $\mathrm{H}$, Holmes KK, Stamm WE. Prevention of pelvic inflammatory disease by screening for cervical chlamydial infection. N Engl J Med. 1996;334(21):1362-6

35. Marrazzo JM, Celum CL, Hillis SD, Fine D, DeLisle S, Handsfield HH. Performance and cost-effectiveness of selective screening criteria for Chlamydia trachomatis infection in women. Implications for a national Chlamydia control strategy. Sex Transm Dis. 1997;24(3):131-41.

36. Brunham RC, Peeling R, Maclean I, McDowell J, Persson K, Osser S. Postabortal Chlamydia trachomatis salpingitis: correlating risk with antigen-specific serological responses and with neutralization. J Infect Dis. 1987;155(4):749-55.

37. 37. Cates W, Jr., Wasserheit JN. Genital 
chlamydial infections: epidemiology and 48. reproductive sequelae. Am J Obstet Gynecol. 1991;164(6 Pt 2):1771-81.

38. Cates W, Jr., Joesoef MR, Goldman MB. Atypical pelvic inflammatory disease: can we identify clinical predictors? Am J Obstet Gynecol. 1993;169(2 Pt 1):341-6.

39. Cumming DC, Honore LH, Scott JZ, Williams KE. Microscopic evidence of silent inflammation in grossly normal fallopian tubes with ectopic pregnancy. Int J Fertil. 1988;33(5):324-8.

40. Jones RB, Mammel JB, Shepard MK, Fisher RR. Recovery of Chlamydia trachomatis from the endometrium of women at risk for chlamydial infection. Am J Obstet Gynecol 1986;155(1):35-9.

41. Paavonen J, Kiviat N, Brunham RC, Stevens CE, Kuo CC, Stamm WE, et al. Prevalence and manifestations of endometritis among women with cervicitis. Am J Obstet Gynecol. 1985;152(3):280-6.

42. Rees $\mathrm{E}$. The treatment of pelvic inflammatory disease. Am J Obstet Gynecol. 1980;138(7 Pt 2):1042-7.

43. Stamm WE, Guinan ME, Johnson C, Starcher T, Holmes KK, McCormack WM. Effect of treatment regimens for Neisseria gonorrhoeae on simultaneous infection with Chlamydia trachomatis. N Engl J Med. 1984;310(9):545-9.

44. Westrom L, Joesoef R, Reynolds G, Hagdu A, Thompson SE. Pelvic inflammatory disease and fertility. A cohort study of 1,844 women with laparoscopically verified disease and 657 control women with normal laparoscopic results. Sex Transm Dis. 1992;19(4):185-92.

45. Haddix AC, Hillis SD, Kassler WJ. The cost effectiveness of azithromycin for Chlamydia trachomatis infections in women. Sex Transm Dis. 1995;22(5):274-80.

46. Makinen J. Ectopic pregnancy falls in Finland. Lancet. 1996;348(9020):129-30.

47. Washington AE, Katz P. Cost of and payment source for pelvic inflammatory disease. Trends and projections, 1983 through 2000. JAMA. 1991;266(18):2565-9.
48. Westrom L, Bengtsson LP, Mardh PA. Incidence, trends, and risks of ectopic pregnancy in a population of women. Br Med J (Clin Res Ed). 1981;282(6257):15-8

49. Westrom LV. Sexually transmitted diseases and infertility. Sex Transm Dis. 1994;21(2 Suppl):S32-7.

50. Brunham RC, Maclean IW, Binns B, Peeling RW. Chlamydia trachomatis: its role in tubal infertility. J Infect Dis. 1985;152(6):1275-82.

51. Cates W, Jr., Rolfs RT, Jr., Aral SO. Sexually transmitted diseases, pelvic inflammatory disease, and infertility: an epidemiologic update. Epidemiol Rev. 1990;12:199-220.

52. Sellors JW, Mahony JB, Chernesky MA, Rath DJ. Tubal factor infertility: an association with prior chlamydial infection and asymptomatic salpingitis. Fertil Steril. 1988;49(3):451-7.

53. Svensson L, Mardh PA, Westrom L. Infertility after acute salpingitis with special reference to Chlamydia trachomatis. Fertil Steril. 1983;40(3):322-9.

54. McCormack WM. Pelvic inflammatory disease. N Engl J Med. 1994;330(2):115-9.

55. Westrom L. Incidence, prevalence, and trends of acute pelvic inflammatory disease and its consequences in industrialized countries. Am J Obstet Gynecol. 1980;138(7 Pt 2):880-92.

56. Reekie J, Donovan B, Guy R, Hocking JS, Kaldor JM, Mak DB, et al. Risk of Pelvic Inflammatory Disease in Relation to Chlamydia and Gonorrhea Testing, Repeat Testing, and Positivity: A Population-Based Cohort Study. Clin Infect Dis. 2018;66(3):437-43.

57. den Heijer CDJ, Hoebe C, Driessen JHM, Wolffs $\mathrm{P}$, van den Broek IVF, Hoenderboom BM, et al. Chlamydia trachomatis and the Risk of Pelvic Inflammatory Disease, Ectopic Pregnancy, and Female Infertility: A Retrospective Cohort Study Among Primary Care Patients. Clin Infect Dis. 2019;69(9):1517-25

58. Land JA, Van Bergen JE, Morre SA, Postma MJ. Epidemiology of Chlamydia trachomatis infection in women and the cost-effectiveness of screening. Hum Reprod Update. 
2010;16(2):189-204

59. van den Broek IV, van Bergen JE, Brouwers EE, Fennema JS, Gotz HM, Hoebe CJ, et al. Effectiveness of yearly, register based screening for chlamydia in the Netherlands: controlled trial with randomised stepped wedge implementation. BMJ. 2012;345:e4316.

60. Low N. Screening programmes for chlamydia infection: when will we ever learn? BMJ. 2007;334(7596):725-8.

61. Rembold CM. Number needed to screen: development of a statistic for disease screening. BMJ. 1998;317(7154):307-12.

62. Steiner RJ, Liddon N, Swartzendruber AL, Pazol K, Sales JM. Moving the Message Beyond the Methods: Toward Integration of Unintended Pregnancy and Sexually Transmitted Infection/ 7 HIV Prevention. Am J Prev Med. 2018;54(3):4403.

63. Bakken IJ, Skjeldestad FE, Lydersen S, Nordbo SA. Births and ectopic pregnancies in a large cohort of women tested for Chlamydia trachomatis. Sex Transm Dis. 2007;34(10):73943.

64. Andersen B, stergaard L, Puho E, Skriver MV, Sch??nheyder HC. Ectopic Pregnancies and Reproductive Capacity After Chlamydia trachomatis Positive and Negative Test Results: A Historical Follow-Up Study. Sexually Transmitted Diseases. 2005;32(6):377-81.

65. Coppus SF, Land JA, Opmeer BC, Steures P, Eijkemans MJ, Hompes PG, et al. Chlamydia trachomatis IgG seropositivity is associated with lower natural conception rates in ovulatory subfertile women without visible tubal pathology. Hum Reprod. 2011;26(11):3061-7.

66. Hay PE, Kerry SR, Normansell R, Horner PJ, Reid $F$, Kerry SM, et al. Which sexually active young female students are most at risk of pelvic inflammatory disease? A prospective study. Sex Transm Infect. 2016;92(1):63-6.

67. Brankovic I, van Ess EF, Noz MP, Wiericx WA, Spaargaren J, Morre SA, et al. NOD1 in contrast to NOD2 functional polymorphism influence Chlamydia trachomatis infection and the risk of tubal factor infertility. Pathog Dis. 2015;73(1):19

68. Morre SA, Karimi O, Ouburg S. Chlamydia trachomatis: identification of susceptibility markers for ocular and sexually transmitted infection by immunogenetics. FEMS Immunol Med Microbiol. 2009;55(2):140-53.

69. den Hartog JE, Lyons JM, Ouburg S, Fennema JS, de Vries HJ, Bruggeman CA, et al. TLR4 in Chlamydia trachomatis infections: knockout mice, STD patients and women with tubal factor subfertility. Drugs Today (Barc). 2009;45 Suppl B:75-82.

70. Rekart ML, Brunham RC. Epidemiology of chlamydial infection: are we losing ground? Sex Transm Infect. 2008;84(2):87-91.

71. Zheng X, O'Connell CM, Zhong W, Nagarajan UM, Tripathy M, Lee D, et al. Discovery of Blood Transcriptional Endotypes in Women with Pelvic Inflammatory Disease. J Immunol. 2018;200(8):2941-56

72. Staritsky, L.E.; F. van Aar; Visser, M; Op de Coul, E.L.M; Heijne, J.C.M.;.; Götz, H.M.; Nielen, M.; van Sighem, A.I.; Benthem, B.H.B Sexually transmitted infections in the Netherlands in 2019. Bilthoven National Institute for Public Health and the Environment 2020, Nr. 20200052.

73. Hocking JS, Temple-Smith M, Guy R, Donovan B, Braat S, Law M, et al. Population effectiveness of opportunistic chlamydia testing in primary care in Australia: a cluster-randomised controlled trial. Lancet. 2018;392(10156):141322

74. Low N, Redmond S, Uuskula A, van Bergen J, Ward $\mathrm{H}$, Andersen $\mathrm{B}$, et al. Screening for genital chlamydia infection. Cochrane Database Syst Rev. 2016;9:CD010866.

75. Dahlberg J, Hadad R, Elfving K, Larsson I, Isaksson $\mathrm{J}$, Magnuson $\mathrm{A}$, et al. Ten years transmission of the new variant of Chlamydia trachomatis in Sweden: prevalence of infections and associated complications. Sex Transm Infect. 2017.

76. Brunham RC, Pourbohloul B, Mak S, White 
R, Rekart ML. The unexpected impact of 80. Sandoz KM, Rockey DD. Antibiotic resistance in a Chlamydia trachomatis infection control Chlamydiae. Future Microbiol. 2010;5(9):1427program on susceptibility to reinfection. $\mathrm{J}$ Infect Dis. 2005;192(10):1836-44.

77. Brunham RC, Rey-Ladino J. Immunology 81. Barbee LA, Khosropour CM, Dombrowksi JC, of Chlamydia infection: implications for a Chlamydia trachomatis vaccine. Nat Rev Immunol. 2005;5(2):149-61.

78. Theunissen KA, Bos AE, Hoebe CJ, Kok G, Vluggen S, Crutzen R, et al. Chlamydia trachomatis testing among young people: what is the role of stigma? BMC Public Health. 2015;15:651.

79. Morre SA, van den Brule AJ, Rozendaal L, Boeke AJ, Voorhorst FJ, de Blok S, et al. The 8 natural course of asymptomatic Chlamydia trachomatis infections: $45 \%$ clearance and no development of clinical PID after one-year follow-up. Int J STD AIDS. 2002;13 Suppl 2:128.

Golden MR. New Human Immunodeficiency Virus Diagnosis Independently Associated With Rectal Gonorrhea and Chlamydia in Men Who Have Sex With Men. Sex Transm Dis. 2017;44(7):385-9.

82. Reitsema $M$, Steffers $L$, Visser $M$, Heijne J, van Hoek AJ, Schim van der Loeff $M$, et al. Cost-effectiveness of increased HIV testing among MSM in The Netherlands. AIDS. 2019;33(12):1807-17.

83. Saag MS, Benson CA, Gandhi RT, Hoy JF, Landovitz RJ, Mugavero MJ, et al. Antiretroviral Drugs for Treatment and Prevention of HIV Infection in Adults: 2018 Recommendations of the International Antiviral Society-USA Panel. JAMA. 2018;320(4):379-96. 



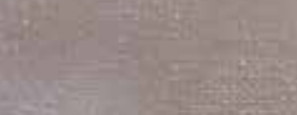

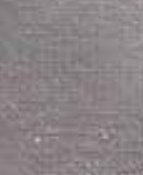

\section{sisting}

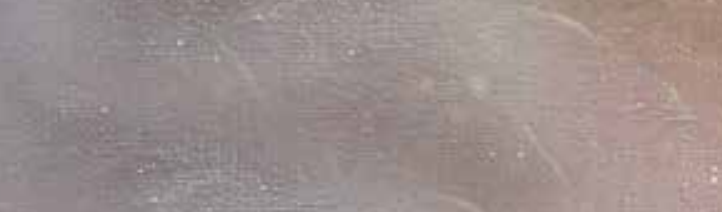

जince

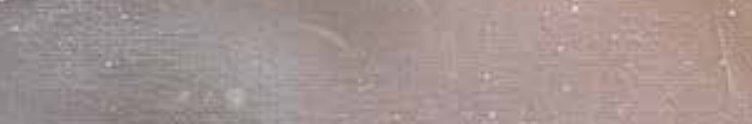

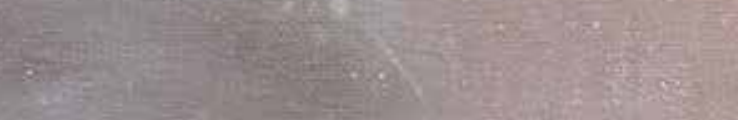

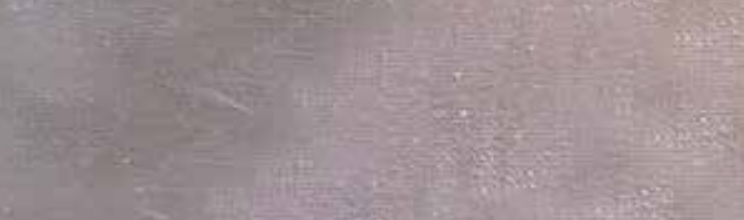

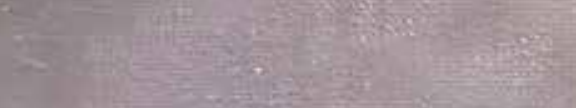

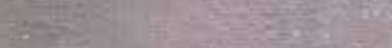

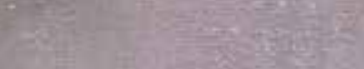

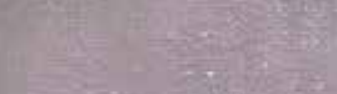

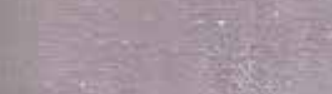

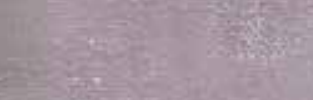

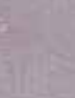

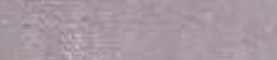

netwo

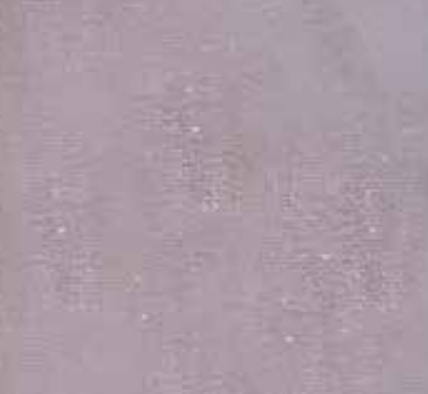

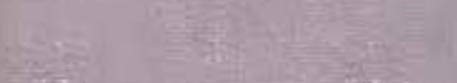

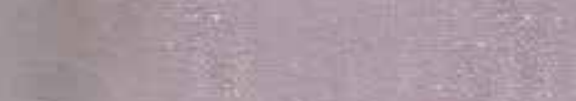




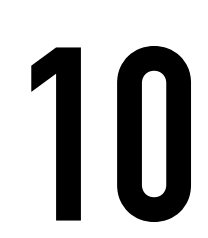

Summary

Samenvatting

Impact Paragraph

Curriculum Vitae

List of publications

Affiliations co-authors

Dankwoord 


\section{Summary}

Chlamydia trachomatis infection ("chlamydia") control is largely focused on detecting and treating asymptomatic infections, limiting ongoing transmission, and thereby reducing the incidence of chlamydia related complications such as pelvic inflammatory disease (PID), ectopic pregnancies and tubal factor infertility (TFI). However, despite the ongoing activities of accelerated testing and treating chlamydia prevalence remains unchanged. Uncertainty exists about the estimates of complications rates following infection as well as determinants associated with developing late complications. To estimate the risk for late complications following a chlamydia infection and to gain insight in factors that contribute to the development of complications, the Netherlands Chlamydia Cohort Study (NECCST) was initiated.

This thesis described research on chlamydia complications and related risk factors in NECCST. Part 1 'The risk of chlamydia for long-term complications: Setting up the Netherlands Chlamydia Cohort study' described and discussed the methodology used for the cohort.

Part 2 focused on 'The value of chlamydia trachomatis serology' in either determining a previous chlamydia infection or as a factor potentially related to disease progression.

Part 3 'Long-term complications of chlamydia infections and predictive factors' described the risk estimates for late complications and pregnancies following chlamydia infection.

In chapter 1, a general introduction is provided on Chlamydia trachomatis, its characteristics, clinical manifestations, potential complications and the epidemiology is described. Furthermore, the challenges of estimating chlamydia related complications are discussed. Studying the natural history of chlamydia comes with many challenges. Due to the asymptomatic nature of the infection, misclassifications of the chlamydia status is common. Case definitions, specifically of PID, are challenging due to unclear diagnostic criteria. A long follow-up is needed because complications of infections often only become apparent after women try to get pregnant, years following infection. And last a large sample size is required because of low incidences of ectopic pregnancies and TFI. With these challenges and the general limitations of cohort studies in mind, NECCST will be 'NECCST best' design in estimating late complications of chlamydia infections.

Part 1 The risk of complications following chlamydia infection: setting up the Netherlands Chlamydia Cohort study

This first part of the thesis focuses on setting up the cohort and the validation studies performed to increase accuracy and reproducibility of the study design. The Netherlands Chlamydia Cohort Study (NECCST) is a longitudinal cohort study following women of reproductive age who previously participated in the Chlamydia Screening Implementation (CSI) study. With NECCST we aimed to estimate the risk for late complications in women with and without a previous infection. Furthermore, the chance of getting pregnant following infection was estimated. Last, determinants associated with developing complications were assessed to help identify women with the highest risk for late complications. Chapter 2 described the study protocol of NECCST. The study started in 2015 and 
will be ongoing until 2022. Information was gathered concerning previous chlamydia infections, late complications, fertility issues, pregnancies and also demographics, sexual risk behaviour and health characteristics. To validate parts of the used methodology, two further studies were performed. Chapter 3 describes a validation method of the use of home collected capillary blood samples for the assessment of chlamydia IgG antibody presence. Validity was high between chlamydia IgG antibody concentration of samples with and without centrifugation delay, therefore this home collection of blood samples introduced a low cost method with simple logistics and is therefore easy to use in large epidemiological studies. Chapter 4 describes a pilot study in which the reuse of previously collected CSI samples for isolating host DNA was tested. Additionally in these samples we tried to confirm the role of four genes by investigating proven associated single nucleotide polymorphisms (SNPs, i.e. TLR2 +2477 G>A, NOD1 + 32656 T- $>$ GG, CXCR5 + 10950 $T>C$, and $I L 10-1082 A>G$ ) in the susceptibility and severity of a chlamydia infection. The majority of previously found associations could not be confirmed. This study underscores the relevance of confirmatory studies and confirmed the use of old biological samples for host DNA isolation.

\section{Part 2 The value of Chlamydia trachomatis serology}

This second part of this thesis focuses on the use and value of chlamydia serology in either identifying people previously infected or to identify antibody patterns associated with chlamydia disease progression. Chapter 5 describes the study of the value of chlamydia serology in addition to using previous NAAT results and self-reported infections in estimating chlamydia lifetime prevalence. Among women with previous negative chlamydia tests, eight percent tested positive for chlamydia antibodies. This significantly increased chlamydia lifetime prevalence with six percent compared to using NAAT results and self-reported infections only. Combining NAAT outcomes, self-reported positive tests, and antibody testing reduced misclassification in chlamydia prevalence estimates. In chapter 6 chlamydia whole-proteome microarrays were utilized to identify antibody patterns associated with infection and with complications in a subsample of NECCST participants. Serum pools were analyzed on whole-proteome arrays. The most reactive antigens were used for further analyses with minimized microarrays. To discriminate between seropositive and seronegative serum samples; a panel of eighteen antigens was created which identified $96 \%$ of all microarray positive samples. Using a whole-proteome array to select antigens for minimized arrays allows for the identification of novel informative antigens as general infection markers. It is however vital to the validate this assay, panel, and potential disease related antigens before it can be used in medical practice.

Overall, chlamydia serology can aid to the surveillance, evaluation of chlamydia control activities and identification of disease specific risk. Current assays however lack sensitivity and specificity in determining population seroprevalence. This reduces the value of chlamydia serology for this purpose. Whole proteome arrays are useful in determining important potential antigens for inclusion in assays which might increase sensitivity and specificity in future assays.

Part 3 Long-term complications of chlamydia infections and predictive factors

In Chapter $\mathbf{7}$ and $\mathbf{8}$ studies were described in which risks and risk factors for PID, ectopic 
pregnancy, TFI and (time to) pregnancies were estimated. About $30 \%$ of 5,704 included women were chlamydia-positive. Incidence rates for all complications were low, between 1.3 and 4.4 per 1,000 person-years. However, the incidence rate of PID was two times higher among chlamydia positives compared to chlamydia negatives. For TFI this was four times higher among chlamydia positives. Incidence rates for ectopic pregnancy were similar. Among chlamydia-positive women, the strongest risk factor for PID was symptomatic versus asymptomatic infection and for TFI this was a young age $(<20)$ at first infection. Overall pregnancy rates were similar in chlamydia positive and negative women, both fifty percent. However, among women with a pregnancy intention, chlamydia positive women became significantly less often pregnant within twelve months than chlamydia negative women. Additionally, pregnancy rates were twenty percent less over time among chlamydia positives $<33$ years of age compared to chlamydia negatives. From these studies it can be concluded that chlamydia-positive women have a considerable higher risk for PID and $\mathrm{TFI}$, and have a longer time to pregnancy. However, the overall incidence rates of complications remained low and ultimately overall pregnancy rates did not differ between chlamydia positive and negative women.

Last, in chapter 9 a general discussion is provided on all topics described above, as well as recommendations for the future. Building on a previous study provided long-term follow up, relevant data and a large sample size that allowed the estimation of chlamydia risk for late complications. The overall risk for complications was low and overall pregnancy rates were similar. Currently in the Netherlands over 60,000 infections are diagnosed and treated yearly without solid evidence that this will decrease chlamydia prevalence or chlamydia complications. A shift from prevention of infection to prevention of complications can be more effective. The ultimate measure will be to have an prognostic tool which differentiates between women with and without an increased risk for late complications. The NECCST cohort will continue to develop a prognostic tool using among other genetic and serology markers. In the meantime different options can explored for testing high risk groups, i.e. high risk for late complications instead of high risk for infections. 


\section{Samenvatting}

Chlamydia trachomatis infectie bestrijding is grotendeels gericht op het diagnosticeren en behandelen van asymptomatische infecties, het verminderen van transmissie en daarmee het verlagen van de incidentie van chlamydia gerelateerde complicaties zoals eileiderontstekingen, buitenbaarmoederlijke zwangerschappen en onvruchtbaarheid veroorzaakt door verklevingen in en rondom de eileiders. Ondanks alle activiteiten om chlamydia transmissie te onderbreken, door het steeds meer testen en behandelen van infecties, blijt de prevalentie van chlamydia in de bevolking onveranderd. Daarnaast is er onzekerheid over hoe vaak complicaties voorkomen na een chlamydia infectie bij vrouwen en welke risicofactoren de kans op complicaties verhogen dan wel verlagen. Om in te kunnen schatten wat het risico van het doormaken van een chlamydia infectie op lange termijn complicaties is en om inzicht te krijgen in de risicofactoren ervan is de Nederlandse Chlamydia Cohort Study (NECCST) opgezet.

Dit proefschrift beschrijt het onderzoek over chlamydia gerelateerde complicaties en geassocieerde risico factoren in NECCST.

Deel 1 'Het risico op chlamydia gerelateerde lange termijn complicaties: Het opzetten van de Nederlandse Chlamydia Cohort Studie' beschrijft en bediscussieert de voor het onderzoek gebruikte methode.

Deel 2 richt zich op de waarde van Chlamydia trachomatis serologie voor het bepalen van een doorgemaakte chlamydia infectie en als mogelijke factor in de ontwikkeling van complicaties.

Deel 3 'Lange termijn complicaties van chlamydia infecties en voorspellende factoren' beschrijft de risicoschattingen voor het ontwikkelen van complicaties en het zwanger worden na een chlamydia infectie.

Het eerste hoofdstuk (Hoofdstuk 1) bestaat uit een algemene introductie over Chlamydia trachomatis, de karakteristieken, het klinisch beeld, mogelijke complicaties en de epidemiologie. Vervolgens worden de uitdagingen van het bepalen van de risico's op chlamydia gerelateerde complicatie uitvoerig besproken. Het bestuderen van het natuurlijke beloop van een chlamydia infectie kent vele uitdagingen. Chlamydia heeft vaak een asymptomatisch beloop waardoor missclassificatie van de chlamydia status veel voorkomt. Definities van complicaties, vooral van PID, zijn lastig door onduidelijk diagnostische criteria. Een lange follow-up is nodig omdat complicaties van chlamydia vaak pas zichtbaar worden zodra vrouwen proberen zwanger te worden, meestal jaren na een infectie. Als laatste is een grote onderzoekspopulatie nodig i.v.m. het weinig voorkomen van buitenbaarmoederlijke zwangerschappen en onvruchtbaarheid (veroorzaakt door eileiderafwijkingen). Door deze uitdagingen, naast de algemene limitaties van cohort studies, heeft NECCST een 'NECCST best' studieopzet in het bepalen van het risico op chlamydia gerelateerde complicaties. 
Deel 1 Het risico op chlamydia gerelateerde lange termijn complicaties: Het opzetten van de Nederlandse Chlamydia Cohort Studie

Het eerste deel van het proefschrift richt zich op het opzetten van het cohort en bijbehorende validatie studies om de validiteit en de betrouwbaarheid van de studie te verhogen. NECCST is een longitudinale cohort studie waarin vrouwen in de reproductieve leeftijd gevolgd worden die eerder hebben meegedaan aan de chlamydia screening implementatie (CSI) studie. Het doel van NECCST is het risico op late complicaties te schatten onder vrouwen met en zonder doorgemaakte chlamydia infectie. Ook de kans op zwangerschap (na een infectie) is geschat. Vervolgens is er gekeken naar factoren die geassocieerd zijn met het ontwikkelen van late complicaties. Met behulp van deze risicofactoren kunnen vrouwen met het hoogste risico op late complicaties geïdentificeerd worden. In hoofdstuk 2 is het studie protocol van NECCST beschreven. Het onderzoek is gestart in 2015 en zal doorlopen tot 2022. Er is informatie verzameld over doorgemaakte chlamydia infecties, late complicaties, vruchtbaarheidsproblemen, zwangerschappen, maar ook demografische factoren, seksueel risicogedrag en gezondheidsdeterminanten. Om de validiteit te bepalen van onderdelen van de gebruikte methode zijn er twee validatiestudies uitgevoerd. Hoofdstuk $\mathbf{3}$ beschrijft de validatie over het gebruik van thuis afgenomen vingerprikbloeddruppels waarin chlamydia antistoffen zijn bepaald. De validiteit was hoog tussen chlamydia immunoglobuline $\mathrm{G}(\mathrm{lgG})$ antistof concentraties in bloedmonsters met en zonder verwerkingsvertraging. Deze thuisafname methode van bloeddruppels introduceert een logistiek gemakkelijke en relatief goedkope methode om bloed te verzamelen in grote epidemiologische studies. Hoofdstuk 4 beschrijft een pilot studie waarin het hergebruik van de eerder verzamelde CSI monsters (vaginale swabs en urine monsters) is getest om humaan DNA uit te isoleren. Daarnaast is in de geteste monsters getracht eerder gevonden associaties te herbevestigen tussen singlepolymorphisms (SNPS) in vier genen en chlamydia vatbaarheid en het verloop van chlamydia infecties. Specifiek ging het om de volgende SNPs: TLR2 + 2477 G >A, NOD1 + 32656 T-> GG, CXCR5 + 10950 T>C, and IL10 -1082 A>G. Humaan DNA bleek goed te isoleren uit de 'oude' verzamelde monsters. Het merendeel van de eerder gevonden associaties kon niet worden bevestigd. Deze studie laat daarmee het belang zien van bevestigingsstudies.

Deel 2 De toegevoegde waarde van Chlamydia trachomatis serologie

Het tweede deel van het proefschrift richt zich op het gebruik en de waarde van chlamydia serologie in enerzijds het identificeren van vrouwen die eerder geïnfecteerd zijn (geweest) met chlamydia en anderzijds in het identificeren van antistofpatronen die geassocieerd zijn met chlamydiapathologie. Hoofdstuk 5 beschrijft de studie waarin de waarde van chlamydia serologie is bepaald als aanvulling op het gebruik van CSI-Nucleic Acid Amplification testen (NAAT) en zelf-gerapporteerde infecties voor het schatten van de chlamydia 'lifetime' prevalentie. Acht procent van vrouwen die eerder NAAT-negatief zijn getest, testten positief op chlamydia antistoffen waardoor de chlamydia lifetime prevalentie met zes procent werd verhoogd. Het combineren van chlamydia serologie, NAAT uitkomsten en zelf gerapporteerde chlamydia infecties reduceerde misclassificatie in chlamydia status. In hoofdstuk 6 is beschreven hoe chlamydia 'whole proteome arrays' gebruikt zijn om te kijken naar associaties tussen antistofpatronen en infecties en antistoffen en chlamydia 
complicaties in een subset van NECCST deelnemers. Serum groepen zijn geanalyseerd op whole-proteome arrays. De meest reactieve antigenen zijn vervolgens gebruikt in de 'minimized microarrays'. Om seronegatieve en seropositieve monsters te onderscheiden is er een panel van achttien antigenen gecreëerd dat uiteindelijk $96 \%$ van alle microarray positieve monsters kon identificeren. Door gebruik te maken van whole-proteome arrays om antigenen te selecteren voor de minimized arrays is het mogelijk om nieuwe informatieve antigenen te selecteren als infectie (-ziekte) markers. Het is zeer belangrijk om de minimized array, het panel van achttien antigenen en de potentieel aan chlamydia complicaties geassocieerde antigenen te valideren alvorens het te gebruiken in de (medische) praktijk.

Chlamydia serologie kan de chlamydia surveillance ondersteunen, het kan gebruikt worden bij de evaluatie van chlamydia control activiteiten en in de identificatie van het risico op chlamydia pathologie. De huidige (commercieel verkrijgbare) testen hebben een onvoldoende hoge sensitiviteit en specificiteit in het bepalen van de populatie prevalentie wat de toegevoegde waarde van chlamydia serologie voor dit doel verlaagt. Whole proteome testen kunnen gebruikt worden om potentiele relevante antigenen voor inclusie in testen te bepalen. Hiermee zou de specificiteit en sensitiviteit van testen verhoogd kunnen worden.

Deel 3 Lange termijn complicaties van chlamydia infecties en voorspellende factoren

In hoofdstuk $\mathbf{7}$ en $\mathbf{8}$ worden de studies beschreven waarin het risico en de risicofactoren zijn bepaald op een eileiderontsteking, buitenbaarmoederlijke zwangerschap, onvruchtbaarheid en (tijd tot) zwangerschap. Ongeveer dertig procent van alle 5704 vrouwen die geïncludeerd zijn in NECCST hebben ooit een chlamydia infectie doorgemaakt. Complicaties kwamen niet vaak voor en de incidenties van alle complicaties waren laag, tussen de 1,3 en 4,4 per 1000 persoonsjaren. De incidentie van een eileiderontsteking was wel twee keer zo hoog voor vrouwen die ooit t.o.v. vrouwen die nooit een chlamydia infectie hadden doorgemaakt. Onvruchtbaarheid, door eileiderafwijkingen, kwam zelfs vier keer zo vaak voor bij vrouwen die ooit een chlamydia infectie hebben doorgemaakt. Incidenties van buitenbaarmoederlijke zwangerschappen verschilden niet van elkaar. Onder vrouwen met en doorgemaakte chlamydia infectie was het hebben van symptomen tijdens een infectie geassocieerd met een verhoogd risico op een eileiderontsteking. Een jonge leeftijd bij de eerste chlamydia infectie ( $<20$ jaar) was geassocieerd met een verhoogd risico op onvruchtbaarheid (door eileiderafwijkingen).

Vijftig procent van alle vrouwen in NECCST zijn ooit zwanger geweest. Hier was geen verschil in tussen vrouwen met en zonder doorgemaakte chlamydia infectie. Echter, onder vrouwen met een zwangerschapsintentie bleken vrouwen met een doorgemaakte chlamydia infectie significant minder vaak zwanger binnen twaalf maanden dan vrouwen zonder doorgemaakte infectie. Uit deze beider studies kan geconcludeerd worden dat een doorgemaakte chlamydia infectie geassocieerd is met een verhoogd risico op een eileiderontsteking, op onvruchtbaarheid (door eileiderafwijkingen) en op een verlengde tijd tot zwangerschap. Maar de incidentie van complicaties is laag en het uiteindelijke aantal vrouwen dat zwanger werd is niet verschillend tussen vrouwen 
met en zonder doorgemaakte infectie.

In hoofdstuk 9 volgt de algemene discussie over alle bovengenoemde studies en onderwerpen als ook de aanbevelingen op basis van dit proefschrift en huidige literatuur. NECCST borduurde voort op de eerder uitgevoerde CSI studie. Deze opzet zorgde voor een lange follow-up van deelnemers, relevante gegevens en een grote onderzoekspopulatie. Hierdoor was het mogelijk om het risico op lange termijn complicaties van een chlamydia infectie te kunnen schatten. Het risico op late complicaties was klein en zwangerschappen kwamen even vaak voor onder vrouwen met een zonder doorgemaakte infectie. Momenteel worden er in Nederland jaarlijks meer dan 60.000 chlamydia infecties gediagnosticeerd en behandeld zonder dat er bewijs is dat dit de prevalentie verlaagt of dat het complicaties voorkomt. Een shift in focus van preventie van chlamydia infecties naar preventie van chlamydia complicaties is een mogelijk effectievere manier van chlamydia bestrijding. Hiervoor is een tool nodig om vrouwen met een verhoogd risico op complicaties te kunnen onderscheiden van vrouwen met een laag risico. Het NECCST cohort zal worden vervolgd met als doel een prognostische tool te ontwikkelen op basis van o.a. genetische kenmerken en serologische markers (ZonMw grant gestart per 1 februari 2021). Totdat een dergelijke tool is ontwikkeld kan worden onderzocht of het mogelijk is om hoog risico groepen specifiek voor chlamydia complicaties te testen i.p.v. hoog risicogroepen voor een chlamydia infectie. 


\section{Impact paragraph}

Chlamydia trachomatis (hereafter referred to as 'chlamydia') is the most common bacterial sexually transmitted infection (STI) among young women. The infection can result in pelvic inflammatory disease (PID), leading to severe complications, such as prolonged time to pregnancy, ectopic pregnancy, and tubal factor infertility. The natural history of chlamydia has been studied for decades, but questions remain and challenges in studying the natural history of chlamydia are not easily resolved. The risk of and risk factors for complications following a chlamydia infection had not been assessed in a long-term prospective cohort study. Therefore, the Netherlands Chlamydia Cohort Study was set up to estimate the risk and risk factors for these reproductive tract problems in women with and without a previous chlamydia infection. Almost 6,000 women participated and were followed for five to ten years. We found that having had a chlamydia infection increased the risk for PID and tubal factor infertility by two to four times and slightly increased the time to pregnancy when compared to women without a previous chlamydia infection. However, complications following chlamydia infections were rare; PID was found in five percent and tubal factor infertility only in one percent of chlamydia positive women. Furthermore, the overall pregnancy chances were similar for women with and without a previous chlamydia infection.

These conclusions together with the set up of the cohort helped in estimating more robust risk estimates for late complications following chlamydia infections. The longitudinal cohort design; building on a previous study (from 2008-2011 that included 60,000 women), long-term follow-up (>ten years) and the extensive manner in which previous chlamydia infections was determined overcome many limitations previously mentioned in studies estimating the risk for late complications following chlamydia infections. Moreover, the results are supportive and contribute highly to the discussion of a new proposed chlamydia control approach concerning testing and treating infections: shifting the focus from preventing chlamydia infections to preventing chlamydia complications. The reason for extensive chlamydia testing is primarily to prevent the spread of chlamydia infections by interfering transmission and to prevent women from severe complications related to a chlamydia infection. But despite a sharp increase in chlamydia testing over the past decades, chlamydia background prevalence remained similar over the last twenty years. PID, ectopic pregnancy and tubal factor infertility is not monitored in a standard and frequent manner, which makes it difficult to assess if the current chlamydia control results in less cases of PID, ectopic pregnancies or tubal factor infertility. In some ecological studies a decline in PID or ectopic pregnancy cases was seen, however declines in these outcomes were also seen in countries with minimum or no systematic chlamydia screening. This information combined with the results from the thesis, which showed low risks for complications, do not support extensive (and therefore expensive) chlamydia testing.

A shift in chlamydia control approach from preventing chlamydia infections to preventing chlamydia complications will have a major impact. Extensive chlamydia testing can be shifted to more targeted chlamydia testing. Targeted testing will be based on risk factors that identify women at highest risk of chlamydia related complications. These risk factors can be based on demographics, infection characteristics, immunological factors and genetic factors. This thesis provides several risk factors 
related to an increased or decreased risk for late complications. When these risk factors can be confirmed and when in future a validated prognostic model will be available, the number of chlamydia tests and treatments will decline drastically and with it the costs. Besides the economic impact the shift will have, it will also have a significant social impact. In the current chlamydia control strategy several risk groups are being tested and treated free of charge. If a shift will be made to only targeted testing for women at high risk for late chlamydia complications, than the criteria and advise for chlamydia testing will change. There will be a lesser need to test boys and men for an infection. And probably many women will not be tested either. Communication will be key during a shift in chlamydia control, in which the low complication rates and similar pregnancy chances for women with and without previous infections will be reassuring for both health care professionals and for girls and women tested positive for chlamydia.

Targeted testing can only be effective if a clear distinction between women with and without high risk for late complications can be made. The Netherlands Chlamydia Cohort Study is further funded until 2025 in which the overall aim is to develop a prognostic tool by which high risk women can be identified. This tool will be piloted to test acceptability and feasibility in which all important stakeholders will be involved. The ultimate aim is to increase effectiveness and efficiency of chlamydia control activities, targeted at high risk women to control chlamydia related complications. 


\section{Curriculum Vitae}

Bernice Hoenderboom was born the $11^{\text {th }}$ of October, 1988 in Winterswijk, the Netherlands. In 2007 she graduated pre-university education from high school at Marianum college in Groenlo after which she shortly studied Life Sciences at the HU university of applied sciences in Utrecht before she switched to studying nursing in Nijmegen at the HAN university of applied sciences. During this study Bernice developed great interest in public health and sexually transmitted infections. Therefore, following her nursing degree, she started with the premaster Health Sciences at the VU university in Amsterdam to study the master Health Sciences with specialization Infectious Diseases and Public Health. She did her

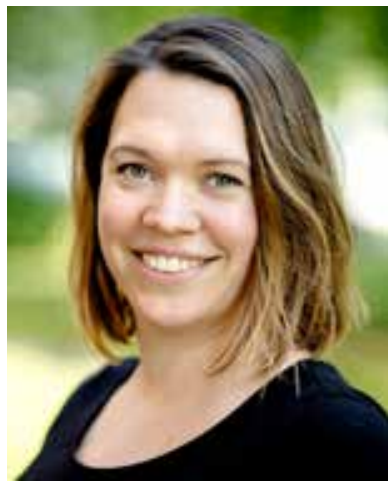
internship at the Amsterdam Institute for Global Health and Development (AIGHD) in Amsterdam about HIV drug resistance in Sub-Saharan Africa. Bernice graduated with honor (cum laude) for her master Health Sciences in 2014. Following graduation Bernice started working as a nurse at the STI clinic at the municipal health service Kennemerland. In June 2015 she started her PhD project at the National Institute for Public Health and the Environment (RIVM), Center for Infectious Disease Control in collaboration with the Amsterdam University Medical Center, location VUmc and later on location AMC. The PhD was finished under supervision of Prof. Dr. Servaas A. Morré, Prof. Dr. Jan EAM van Bergen, Dr. Birgit HB van Benthem, and Dr. Sander Ouburg. The PhD topic was about estimating the risk of long-term complications following Chlamydia trachomatis infection in women. Currently she works at the HAN university of applied sciences as a teacher in nursing and in February 2021 she will combine this post with a postdoc on the Netherlands Chlamydia Cohort Study at the Maastricht University Medical Center and the RIVM. 


\section{List of publications}

- Jukema JB, Hoenderboom BM, van Benthem BHB, van der Sande MAB, de Vries HJC, Hoebe CJPA, Dukers-Muijrers NTHM, Bax JC, Morré SA, Ouburg S. Can Previous Associations of Single Nucleotide Polymorphisms in the TLR2, NOD1, CXCR5, and IL10 Genes in the Susceptibility to and Severity of Chlamydia trachomatis Infections Be Confirmed? Pathogens 2021;10(48).

- Hoenderboom BM, van Bergen J, Dukers-Muijrers N, Gotz HM, Hoebe C, de Vries HJC, et al. Pregnancies and Time to Pregnancy in Women With and Without a Previous Chlamydia trachomatis Infection. Sex Transm Dis. 2020;47(11):739-47.

- Hufnagel K, Hoenderboom BM, Harmel C, Rohland JK, van Benthem BHB, Morre SA, et al. Chlamydia trachomatis Whole-Proteome Microarray Analysis of The Netherlands Chlamydia Cohort Study. Microorganisms. 2019;7(12).

- $\quad$ Hoenderboom BM, van Willige ME, Land JA, Pleijster J, Gotz HM, van Bergen J, et al. Antibody Testing in Estimating Past Exposure to Chlamydia trachomatis in the Netherlands Chlamydia Cohort Study. Microorganisms. 2019;7(10).

- $\quad$ den Heijer CDJ, Hoebe C, Driessen JHM, Wolffs P, van den Broek IVF, Hoenderboom BM, et al. Chlamydia trachomatis and the Risk of Pelvic Inflammatory Disease, Ectopic Pregnancy, and Female Infertility: A Retrospective Cohort Study Among Primary Care Patients. Clin Infect Dis. 2019;69(9):1517-25.

- Hoenderboom BM, van Benthem BHB, van Bergen J, Dukers-Muijrers N, Gotz HM, Hoebe $\mathrm{C}$, et al. Relation between Chlamydia trachomatis infection and pelvic inflammatory disease, ectopic pregnancy and tubal factor infertility in a Dutch cohort of women previously tested for chlamydia in a chlamydia screening trial. Sex Transm Infect. 2019;95(4):300-6.

- Hoenderboom BM, van Ess EF, van den Broek IVF, van Loo IHM, Hoebe C, Ouburg S, et al. Chlamydia trachomatis antibody detection in home-collected blood samples for use in epidemiological studies. J Microbiol Methods. 2018;144:164-7.

- Hoenderboom BM, van Oeffelen AA, van Benthem BH, van Bergen JE, Dukers-Muijrers NH, Gotz HM, et al. The Netherlands Chlamydia cohort study (NECCST) protocol to assess the risk of late complications following Chlamydia trachomatis infection in women. BMC Infect Dis. $2017 ; 17(1): 264$.

- $\quad$ Boender TS, Hoenderboom BM, Sigaloff KC, Hamers RL, Wellington M, Shamu T, et al. Pretreatment HIV drug resistance increases regimen switches in sub-Saharan Africa. Clin Infect Dis. 2015;61(11):1749-58. 


\section{Affiliation list}

Authors

Dr. Bax, Caroline $\mathrm{J}^{1}$

Prof. Dr. de Vries, Frank2,3,4

Prof. Dr. de Vries, Henry JC5,6

Dr. Dukers-Muijrers, Nicole HTM ${ }^{7,8}$

Dr. Götz, Hannelore M9,10,11

Harmel, Christoph, BSc.s ${ }^{12}$

Prof. Dr. Hoebe, Christian JPA ${ }^{7,8}$

Dr. Hogewoning, Arjan $A^{6}$

Dr. Hufnagel, Katrin ${ }^{12}$

Jukema, Jelmer B, MSc. ${ }^{13}$

Dr. Land, Jolande $\mathrm{A}^{14}$

Prof. Dr. Morré, Servaas $A^{13,14}$

Dr. Ouburg, Sander ${ }^{13}$

Pleijster, Jolein, Ing ${ }^{13}$
Rohland, Juliane K, BSc. ${ }^{12}$

Prof. Dr. van Baarle, Debbie ${ }^{15}$

Dr. van Benthem, Birgit HB $^{9}$

Prof. Dr. van Bergen, Jan EAM $9,16,17$

Dr. van den Broek, Ingrid VF ${ }^{9}$

Prof. Dr. van der Klis, Fiona RM ${ }^{18}$

Prof. Dr. van der Sande, Marianne $A^{19,20}$

Dr. van Ess, Eleanne $\mathrm{F}^{13}$

Dr. van Loo, Inge $\mathrm{HM}^{8}$

Dr. van Oeffelen, Louise $A^{9}$

Dr. van Veen, Maaike $\mathrm{G}^{6}$

van Willige, Michelle E, MSc. ${ }^{9}$

Dr. Waterboer, Tim ${ }^{12}$

\section{Affiliations}

1. Department of Obstetrics and Gynaecology, Amsterdam UMC, location AMC, University of Amsterdam, The Netherlands

2. Division of Pharmacoepidemiology and Clinical Pharmacology, Utrecht Institute of Pharmaceutical Sciences, Heerlen, The Netherlands

3. Department of Clinical Pharmacy and Toxicology Maastricht UMC+, Maastricht, The Netherlands

4. CARIM, School for Cardiovascular Diseases, Maastricht UMC+, Maastricht, The Netherlands

5. Amsterdam UMC, University of Amsterdam, Department of Dermatology, Amsterdam Institute for Infection and Immunity (AI\&II), location Academic Medical Centre, Amsterdam, The Netherlands

6. STI outpatient clinic, Department of Infectious Diseases, Public Health Service Amsterdam, The Netherlands

7. Department of Sexual Health, Infectious Diseases and Environmental Health, South Limburg Public Health Service (GGD South Limburg), Geleen, The Netherlands

8. Department of Social Medicine and Medical Microbiology, Care and Public Health Research Institute (CAPHRI), Maastricht University, Maastricht, The Netherlands

9. Epidemiology and Surveillance Unit, Centre for Infectious Disease Control, National Institute for Public Health and the Environment, Bilthoven, The Netherlands

10. Department of Infectious Disease Control, Municipal Public Health Service RotterdamRijnmond (GGD Rotterdam), Rotterdam, The Netherlands

11. Department of Public Health, Erasmus MC-University Medical Center Rotterdam, Rotterdam, The Netherlands 
12. Infections and Cancer Epidemiology, German Cancer Research Center (Deutsches Krebsforschungszentrum, DKFZ), Heidelberg, Germany;

13. Laboratory of Immunogenetics, Department Medical Microbiology and Infection Control, Amsterdam UMC, Location AMC, Amsterdam, The Netherlands.

14. Department of Genetics and Cell Biology, Research School GROW (School for Oncology \& Developmental Biology), Faculty of Health, Medicine \& Life Sciences, University of Maastricht, Maastricht, The Netherlands

15. Department Immune mechanisms, center for Infectious Disease control, National Institute for Public Health and the Environment, Bilthoven, The Netherlands

16. Department of General Practice, Division Clinical Methods and Public Health, Academic Medical Center, Amsterdam The Netherlands

17. STI AIDS Netherlands (SOA AIDS Nederland), Amsterdam, The Netherlands

18. Laboratory for Infectious Diseases and Perinatal Screening, Centre for Infectious Disease Control, National Institute of Public Health and the Environment, Bilthoven

19. Julius Center for Health Sciences and Primary Care, University Medical Center Utrecht, Utrecht, The Netherlands

20. Institute of Tropical Medicine, Department of Public Health, Antwerp, Belgium 


\section{Dankwoord}

En dan is daar het moment dat je mag gaan zitten voor het schrijven van je dankwoord en eindelijk iedereen mag bedanken die een directe dan wel indirecte bijdrage heeft geleverd aan dit proefschrift. Vreselijk spannend, maar het voelt vooral als een eer. Velen hebben niet alleen inhoudelijk bijgedragen, maar hebben er ook voor gezorgd dat de tijd van mijn promotieonderzoek werkelijk fantastisch was. Te beginnen met mijn promotieteam.

Beste Servaas, het is al vaker gezegd, maar dat maakt het niet minder waar: Je bent werkelijk de meest enthousiaste promotor die ik me kan voorstellen. Je ziet overal kansen en mogelijkheden en problemen zijn er om op te lossen. Als ik graag een extra onderzoek wilde uitvoeren of een samenwerking wilde aangaan was de vraag niet of ik dat kon doen, maar hoe ik dat kon aanpakken. En dan je ongekende bereikbaarheid. Vakantie betekende voor jou een wisseling van werkplek. Op een memorabele dag ergens in mijn eerste maanden als promovendus was jij op vakantie in Spanje. Die ochtend mailden we wat heen en weer over niet-dringende zaken. In de middag kreeg ik een mail van je: "Ik ben even niet bereikbaar i.v.m. een excursie, over twee uur ben ik weer terug." Ik heb me serieus afgevraagd wat er van mij werd verwacht zodra ik op vakantie zou gaan. Toch was deze ongekende bereikbaarheid bijzonder prettig of dit nu per telefoon, per app of per email was. Bijvoorbeeld toen ik er op Koningsdag achter kwam dat ik mijn manuscript bij een verkeerd journal had ingediend en lichtelijk in paniek was, kon ik je gewoon bereiken. Je lachte, stelde me gerust, we bedachten een oplossing en iedereen kon weer verder met zijn dag. Ontzettend bedankt voor je vertrouwen, de begeleiding en kansen tijdens mijn promotieonderzoek en voor de mooie mogelijkheid om nu als postdoc bij jou aan de slag te gaan.

Beste Birgit, ik wil je ten eerste heel erg bedanken dat je mij de kans hebt gegeven om te starten als PhD student bij jou op de soa afdeling. Naast dat je mijn leidinggevende was en de projectleider van NECCST werd je, ongeveer twee jaar nadat ik begonnen was, ook mijn copromotor. Ik vond de samenwerking hierin erg prettig. Onze gesprekken over de studie, de analyses, de resultaten waren altijd zeer levendig met vele zijpaadjes (over van alles en nog wat) en we liepen eigenlijk altijd uit. Daarnaast bewonder ik je nuchtere en praktische kijk op zaken. Ik ben met enige regelmaat gestrest bij je kantoor binnen gelopen om binnen enkele minuten met een gerust hart weer naar buiten te komen. Maar daarnaast ook bedankt voor alle gezelligheid en lachen bijvoorbeeld tijdens avondjes uit midden in Ipanema in Rio de Janeiro.

Beste Jan, in het begin van mijn promotieonderzoek hebben we je gevraagd om als tweede promotor deel te nemen in mijn promotieteam. Jouw ervaring op het gebied van chlamydia en publieke gezondheid was een ontzettend waardevolle aanvulling tijdens mijn onderzoek en het interpreteren van alle resultaten. Je kritische houding t.o.v. het huidige chlamydia beleid heeft veel stof gegeven tot nadenken. Tijdens onze gesprekken samen op het RIVM had je niet alleen aandacht voor het inhoudelijke, maar vroeg je altijd even hoe het ging en hoe ik de promotie en alle onderdelen ervaarde. Bedankt! 
Beste Sander, jij was het eerste aanspreekpunt binnen het VUmc voor mij als copromotor en later binnen het Amsterdam UMC. Vele verhuizingen hebben we meegemaakt en jij was diegene die iedereen rondleidde, op het gemak liet voelen en kennis leerde te maken met het lab. Heel erg bedankt dat ik altijd binnen kon lopen als ik ergens tegenaan liep, of dit nu ging om host genetica, serologie of logistieke vragen rondom de studie.

Beste Ingrid en Louise, hoewel jullie inmiddels (al jaren) niet meer bij het RIVM werken, waren jullie diegene die mij hebben ingewerkt op het NECCST onderzoek. Ingrid jij bent diegene die begonnen is met het opzetten van NECCST en het schrijven van alle aanvragen. Louise, jij hebt veel gedaan in de methodologie en de voorbereiding op de studie. Ontzettend bedankt voor jullie enthousiasme, jullie hulp en begeleiding en voor de inspiratie voor dit proefschrift.

Geachte leden van de beoordelingscommissie, Prof. dr. P.H.M. Savelkoul, Prof. dr. R.F.P.M Kruitwagen, Prof. dr. D.D.M. Braat en Dr. Y. Pannekoek bedankt voor het lezen en beoordeling van mijn proefschrift.

Een dergelijke grote en ingewikkelde studie als NECCST kan niet zonder een goede adviescommissie. Beste Prof. dr. Marianne van der Sande, Prof. dr. Christian Hoebe, Dr. Nicole Dukers-Muijrers, Dr. Hannelore Götz, Dr. Arjan Hogewoning, Dr. Jolande Land, Prof. dr. Frank de Vries, Prof. dr. Henry de Vries ontzettend bedankt voor het kritisch doch enthousiast meedenken met alle studieperikelen, het meedenken en geven van feedback op manuscripten en vooral ook voor de interessante discussies en de motivatie die dit mij iedere keer weer gaf. Dank ook aan alle coauteurs die de manuscripten weer tot een hoger niveau tilden.

Ook een grote dank aan alle NECCST studiedeelneemsters.

Mijn paranimfen Merel en Daphne. Ik vond het een eer paranimf bij jullie te mogen zijn en ik ben ontzettend blij en opgelucht dat jullie mij straks bijstaan. Lieve Merel, al zo lang vriendinnen en bijna gelijktijdig begonnen we met een promotieonderzoek. Voor lange tijd ook aan dezelfde universiteit. Wat was het fijn om dit samen te kunnen doen. De koffiepauzes in en rondom de VU waren altijd broodnodig. Was het niet om successen te bespreken dan vooral om lekker te klagen over hoe het allemaal ging. Al kon dit overigens ook prima in de nabijgelegen sauna of onder het genot van sushi. Bedankt lieverd dat je, waar het ook over ging, altijd vierkant achter me stond!

Lieve Daphne, ik kan mijn promotieonderzoek niet los zien van jou. Ik vond het fantastisch om zo gelijktijdig met jou te kunnen promoveren. Zoals jij het al verwoordde ontstond er al snel een hele mooie vriendschap. En wat hebben we een gave dingen meegemaakt, in het prachtige Oxford, het mooie en enge Rio en toen nog naar Vancouver. Regelmatig sta ik nog stil voor een 'Daphne-momentje'. Maar misschien wel de fijnste momenten waren de 'goeie koffie pauzes' op het RIVM, het liefst buiten in de zon. Bedankt voor al het lachen, luisteren, de gezelligheid en het er zijn! 
Graag wil ik ook mijn (oud) collega's van het RIVM bedanken. Janneke H, Fleur, Maartje, Chantal, Eline Hannelore en Laura en nu ook de nieuwe soa collega's, bedankt voor alle interessante discussies, het meedenken met mijn onderzoek, maar vooral ook de gezelligheid binnen de soa groep de afgelopen jaren. Zoïe, jij neemt het stokje van mij over als PhD student op NECCST en ik heb er alle vertrouwen in dat we vele mooie onderzoeken tegemoet gaan zien, veel succes! Petra, Daphne en Maartje bedankt voor de gezellige koffiepauzes en marktuitjes waar we altijd heel fijn onze PhD of werkzaken met elkaar konden bespreken. Lieve Maarten, alleen al als ik aan je denk hoor ik je lach en waarschijnlijk de rest van de afdeling ook. Als jij er was kon mijn dag al niet meer stuk. Wat is het fijn je nu weer af en toe te zien! Mijn kamergenoten Irene, Pim, Abby, Janneke en Emma, ik vond het altijd erg gezellig met jullie op de kamer. Alle leden van de feestcommissie, Tom, Maartje, Maarten, Marit, Daphne en Irene, bedankt voor al het lachen, de gezelligheid, de verkleedpartijen en vooral de borrels! Janneke $V$ en Joram en andere 'derde verdiepingers' bedankt voor alle gezelligheid. De stagiaires bij de soa afdeling, Maud en Michelle. Maud altijd even enthousiast en assertief en Michelle heel nauwkeurig en betrokken, bedankt voor jullie inzet en top bijdrage aan twee verschillen onderzoeksprojecten. Het secretariaat van de afdeling EPI en in het bijzonder Ilse en later Saskia, ontzettend bedankt voor jullie hulp rondom alle logistiek van NECCST en uiteraard de gezellige praatjes.

Twee dagen per week mocht ik aan de slag bij het VUmc, laboratorium immunogenetica. En ook daar wil ik graag mijn collega's bedanken. Eerst mijn medepromovendi. Lieve Ea, jij maakte echt mijn tijd op het VUmc. Twee dagen waren eigenlijk te weinig om bijgepraat te raken, alles was bespreekbaar! Van chlamydia onderzoek tot borstvoeding. Bedankt voor alle gezelligheid, jouw fijne en nuchtere kijk op het leven en uiteraard je bijdrage aan dit proefschrift. Martin jouw droge humor was een welkome afwisseling. En hoewel niet altijd even stipt, was je er altijd. Je stond altijd klaar om te helpen, mee te denken of gewoon om te luisteren. En je filmavonden met de meest vreselijkste films, waren fantastisch. Bedankt! Lieve Dewi als iemand weet wat gezelligheid is ben jij het, maar ik kon je ook altijd om hulp vragen. En damn wat pippeteer jij snel! Bedankt voor de leuke tijd, zowel in de $\mathrm{O} \mid 2$ toren als op congressen. Lieve Omaima, we hebben tijdens ons $\mathrm{PhD}$ traject beiden ons eerste kindje gekregen en dat maakte dat we ons heel goed konden inleven in hoe de combinatie was. Jij ook nog veel succes met het afronden van je proefschrift.

Beste Jolein, vooral jij maakte me wegwijs in het lab op het VUmc. Met name de chlamydia ELISA's zijn we vol ingedoken. Ontzettend bedankt ook voor al het harde werk op de letterlijk duizenden NECCST serum samples! Een ongelooflijk waardevolle bijdrage aan mijn proefschrift. Roel ook jij stond altijd klaar om te helpen of om iets uit te leggen. Het liefst met mooie hitjes op de achtergrond wat het werken op het lab altijd een stuk gezelliger maakte.

De stagiaires op het VUmc, Vincent en Yolanda, bedankt voor jullie harde werk. En Jelmer! Ik weet niet waar we meer over geleerd hebben: inhoudelijk over SNPs en chlamydia of het hebben van heel veel geduld en nog meer doorzettingsvermogen. Maar het resultaat mag er wezen! Maar ook alle andere collega's waar ik langer of korter mee heb samen mogen werken Anne, Melisa Mansi, Wies, June, Naomi, Esmee en Jan-Henk, bedankt voor de gezellige werksfeer. 
Dear Katrin, we met in Oxford at the ESCR, which turned out to be a great start of our collaboration. I really enjoyed working with you. Especially when you came to Amsterdam and even more so when you welcomed me in Heidelberg at the DKFZ, many thanks for the hospitality!

Mijn nieuwe collega's op de Hogeschool Arnhem en Nijmegen bedankt voor jullie interesse in mijn onderzoek.

Mijn lieve vrienden en vriendinnen, in het bijzonder Elze, Eefje, Elske, Evelien, Floor, Lisa, Loes, Malou, Marleen, Merel, Nanne, Nicole en onze fantastische aanhang bedankt voor alle borrelavonden, eetclubs, de kroeg in, of de tegenwoordige babyshowers, luiertaarten maken of wat dan ook, dit zijn echt de noodzakelijke afleidingen die horen bij een PhD onderzoek. Sabine en Erik, soms is het juist fijn om wel alle proefschrift perikelen met elkaar te kunnen delen!

Lieve pap en mam, of jullie altijd precies wisten waar ik mee bezig was, dat denk ik niet. Maar dat jullie trots waren op alles wat ik deed was duidelijk! Jullie hebben me altijd vrij gelaten om zelf te kiezen wat ik wilde gaan doen. Ook al veranderde ik weleens van richting gaandeweg. Daarnaast stonden jullie altijd klaar om te helpen met onze huizen of het oppassen op de kinderen, wat mij weer ruimte gaf om met een gerust hart te gaan werken. Ontzettend bedankt hiervoor! Ook Wiebe en Jelke, Ivanka en Sjaak, Wim en Ineke en verdere schoonfamilie. Bedankt voor de interesse, het oppassen, het aanhoren van alle proefschrift verhalen en het brengen van afleiding .

En dan als laatste mijn fantastische man. Lieve Thomas, jij weet als geen ander hoe dit traject voor mij geweest is, want je staat echt altijd voor me klaar. Sinds het moment dat we elkaar leerden kennen staan we samen. En dat bleek ook echt de afgelopen jaren. Niet alleen zijn we getrouwd, ook hebben we onze twee fantastische bengels Ted en Kato gekregen. Een hele hoop avonturen die ik alleen maar met jou wilde beleven. Ik ben zo ontzettend gelukkig met je en ik kijk uit naar alles wat nog komen gaat. Bedankt lieverd! 


